\title{
PROPOSAL FOR A \\ DEDICATED COLLIDER AT THE FERMI NATIONAL \\ ACCELERATOR LABORATORY
}

MAY, 1983

Operated by the Universities Research Association, Inc. under contract with the U.S. Department of Energy 
FERMILAB DEDICATED COLLIDER

TABLE OF CONTENTS

Preface

I. Introduction

II. Physics

III. The $p \vec{p}$ Collider

IV. Proton Ring Components

v. The ep Collider

VI. Conventional Construction and Site Considerations

VII. Costs

VIII. Project Management and Staffing

IX. Parameter Optimization

Executive Summary 

Preface

I. Introduction
A. The Big Picture
B. Why an "intermediate range" ring at Fermilab?
C. Why use"conventional" superconducting technology?
D. Why ep/pp collisions?
E. Is there scientific justification?

II. Pnysics
A. Beyond the Bellwethers
B. Electroweak Phenomena
C. Hadron Jets
D. New Landmarks in 1-TeV Physics
1. Technicolor
2. Supersymmetric Partners of the Known Particles
3. Compositeness

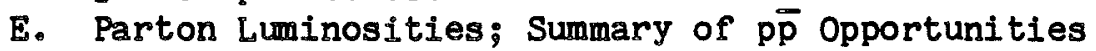

F. Uncertainties in Rate Estimates

G. Electron-Proton Physics

1. Standard Physies: QCD

2. Standard Physics: Electroweak Fhenomena

3. Extensions of the Standard Model

4. Other Possibilities

5. Summary

III. The pp Collider

A. Design Goals and Constraints

B. The Injector

C. Ring Lattice

1. General description and parameters

2. The low $\beta$ zero-n insertion

3. Beam separation

4. Correction magnets

D. Injection, Extraction and Abort Systems

1. Extraction from Tevatron

2. Beam Transport and Injection into DC

3. Beam Extraction from DC

4. Beam Abort System

E. Radiof requency System

F. Performance

1. Single Beam Instabilities

2. Luminosity

3. Beam-beam limits and luminosity lifetime

G. Operating scenarios

H. Bunch-Avoidance Beam Dynamics 
IV. Proton Ring Components
A. Magnets
B. Refrigeration System
C. Vacuum System
D. Control System
E. RF System

V. Electron Ring
A. Luminosity
B. Polarization
C. Lattice
D. Interaction regions
E. Vacuum system
F RF
G. Magnets
H. Injection
I. Future upgrading

VI. Conventional Construction and Site Considerations

A. Site geology; topography and subsurface features

B. Special problems

1. Lake Law

2. Indian Creek

3. Neutrino Area

4. Mt. Minooka

C. Tunnel

D. Experimental areas

E. Miscellaneous enclosures

1. Utility Straight Sections

2. Cryogenic Service Buildings

3. Emergency Exits

4. Modification of Straight Section E

F. Roads and Services

1. Roads

2. Utility Distribution

3. Office and shop space

G. Environmental, health and safety considerations

VII. Costs
A. Magnets
B. Refrigeration System
C. Conventional Construction
D. Accelerator Systems
E. Testing and Installation
F: R\&D Costs
G. The ep Facility
H. Operating Costs 
VIII. Project Management and Staffing

A. Laboratory Priorities

B. Design \& Construction Profile

C. General Comments

D. Management Structure

E. Profile of Staffing Requirements

F. Requirements on A\&E Effort

G. Funding Profile

IX. Parameter Optimization

Executive Summary 
. 
This document describes a proposal for a proton-antiproton/ electronmproton Dedicated Collider facility at Fermilab, to be built between 1985 and 1989. The centermof mass energy of the pp collider is more than $4 \mathrm{TeV}$ and the (Stage I) ep facility provides collisions of a $10 \mathrm{GeV}$ electron beam on the $2-\mathrm{TeV}$ proton beam $\left(s=80000 \mathrm{GeV}^{2}\right)$. An increase of the electron energy to $40 \mathrm{GeV}$ is a natural second-stage project for a later date.

The Dedicated Collider ring fits gracefully within the present Fermilab site and can make use of the Tevatron as an injector for both protons and antiprotons. It also makes use of existing Fermilab superconducting-magnet and refrigeration-system technology to provide a rapid and economical way to more than double the maximum US and, indeed, world hadron collider energy. This very high energy, good luminosity, and large available running time will increase the potential physics productivity of the US program by orders of magnitude. The Stage I ep option is competitive with any existing or planned ep facility and Stage II would be unique in the world.

The Dedicated Collider is designed to have a total of six experimental areas, four major experimental areas for $\bar{p} \bar{p}$ collisions and two for ep collisions. Further, the Tevatron fixed-target program will no longer share the accelerator with the $\mathrm{TeV} I$ collider and can therefore operate at full efficiency. The $p \bar{p}$ luminosity is in excess of $10^{31} \mathrm{~cm}^{-2} \mathrm{sec}^{-1}$ and the ep luminosity is $6 \times 10^{31} \mathrm{~cm}^{-2} \mathrm{sec}=1$. The first stage of this project can be chosen to emphasize either the ep option or the $\mathrm{p} \overrightarrow{\mathrm{p}}$ option, depending upon the scientific priorities perceived to exist at the time of decision (approximately 1986). No matter which option is chosen, it will be laboratory policy that the fixed-target Tevatron II physics program wil1 not be compromised either in support or in operation by the construction program for the Dedicated collider.

At present Fermilab is deeply committed to commissioning the Tevatron and building the Tevatron I and Tevatron II projects. This work will keep the Laboratory absorbed for several years. However, now that the Tevatron program is well defined, it is appropriate to consider the future direction of the Laboratory in the context of scientific needs and world activities. Indeed, the existence this year of a HEPAP Subpanel on Long Range Planning makes it important, some would say obligatory, to provide to the community Fermilab's ideas on Ionger-range planning options.

The 1981 Subpanel on Long Range Planning, chaired by G. Trilling, recommended "a start by the mid 1980's on a new high energy construction project...". Examples of such a new facility cited in that report are nan electron-proton collider or a less expensive high luminosity ( $\mathrm{L}^{-1} 10^{3} \mathrm{~cm}^{-2} \mathrm{sec}^{-1}$ ) hadron-hadron collider built in the ISABELLE tunnel; a second proton collider ring at Fermilab dedicated to $p \bar{p}$, pp, and/or ep collisions, an $e^{+} e^{-}$collider using superconducting cavities (as proposed 
for CESR II), or a combination of smaller facilities, one of which might be a major non-accelerator facility."

The Fermilab Dedicated Collider provides an excellent, practical solution to this perceived need for a new facility. But most important, it will produce the first-class science that is required by the rapid evolution of the field. By 1989, there will be great pressure to explore physics at multi-TeV energies beyond $\mathrm{TeV} I$.

The first chapter dicusses how the Dedicated Collider fits into the international program in high-energy physics, and how it appears to be an optimal choice for Fermilab as well. Subsequent chapters of this document describe the scientific goals, detailed ring design and performance, costs, manpower estimates, and funding profiles for the Dedicated Collider. If the busy reader is already convinced of the scientific merit of this proposal, he can skip ahead to Table VIII-1 for an overview of funding, schedule, and manpower deployment. In Chapter IX we discuss design improvement options which can reduce costs or increase the energy to $-5 \mathrm{TeV}$.

The basic philosophy underlying the design is to minimize research and development and capitalize on the large and successful Fermilab R\&D programs of the past several years. In this way, the Dedicated Collider can be built rapidly, using designs and estimates of costs and schedules based on actual experience.

Finally we note that the Dedicated Collider is an evolution of the Fermilab Site Filler which has been part of the Laboratory's long-range planning since - 1972. In 1974 Robert Wilson wrote in Scientific American (230, 72,1974$)$ :

\footnotetext{
"The largest superconducting ring we could build within our present boundaries would have a circumference of about 10 miles. If the facility were designed as an intersecting-storage-ring system, it might enable us to reach collision energies of several million GeV. If the experiments we are now capable of doing do not yield the knowledge we desire, or, what is more likely, if the new knowledge makes it irresistible to discover what happens at very much higher energies, we are confident those energies can be achieved at our laboratory on the IIlinois plain."
} 



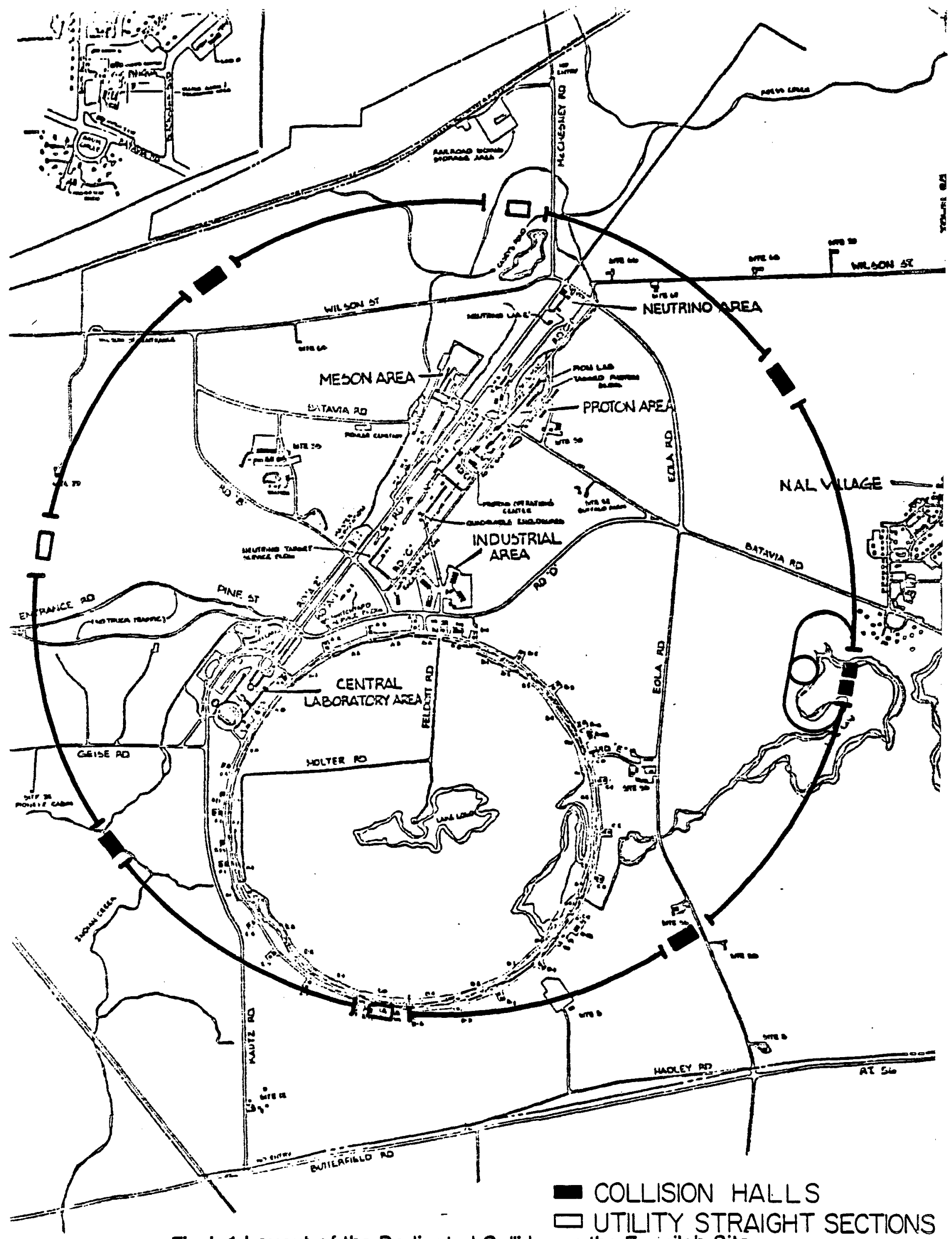

Fig. $1-1$ Layout of the Dedicated Collider on the Fermilab Site 


\section{INTRODUCTION}

\section{A. The Big Picture}

Following the successful operation of the CERN Spps collider, the commissioning of $\mathrm{TeV} I$ in $1985-1986$ will open the era of hadron-collision physics at the TeV scale and of particle physics beyond the electroweak scale of $100 \mathrm{GeV}$ cms energy. Results of the work in this range will thereafter increasingly draw attention to even higher energies. There will be a clear scientific need to extend the exploration of multi-TeV hadron collisions beyond $\mathrm{TeV} I$ during the decade 1985-1995. To meet this need, a new hadron-hadron collider ought to be commissioned well before 1995.

Fermilab proposes to build a Dedicated Collider (DC) facility available for physics before the end of 1989. This facility, shown in Fig. I- 1 , will provide $p \bar{p}$ collisions at center-of-mass energies in excess of $4 \mathrm{TeV}$ with luminosities larger than $10^{31} \mathrm{~cm}^{-2} \mathrm{~s}^{-1}$ and ep collisions between $10 \mathrm{GeV}$ electrons and $2 \mathrm{TeV}$ protons with a luminosity of $6 \times 10^{31} \mathrm{~cm}^{-2} \mathrm{~s}^{-1}$. How might this DC fit into the world program in high energy physics?

In the Soviet Union there is the $3 \times 3 \mathrm{TeV}$ collider UNK, with a very uncertain completion date. Upon completion, there is an even greater uncertainty whether the Soviet Union or an international user community can mount the complex experimental effort needed to extract the physics results in a timely way.

European efforts in High Energy Physics in the 80 's display an unprecedented vigor with the construction of LEP, the thrust to achieve ep collisions at HERA and the already operating spps collider. There is also the prospect of ultimately filling the LEP tunnel with a pp collider which would achieve $2 \mathrm{TeV}$ in the CM for every tesla of magnetic field. Thus, for example, a Saver-type magnet would reach $4.5 \mathrm{TeV}$ per beam. However, this effort would conflict with LEP II, the unique facility to probe beyond the $\mathrm{z}^{\circ}$ with $e^{+} e^{-}$collisions, and it would be as costly as the entire LEP project. Thus it is unlikely that such a facility would appear in the early 90 's.

What can the US provide in the decade after experiments begin at TeV I? Fermilab offers this proposal, a proton-antiproton collider which we believe will reach cm energies close to $5 \mathrm{TeV}$ before the end of the decade and with minimal technical risk. There is an alternative, the "desertron" initiative, a hadron collider reaching $10 \times 10 \mathrm{TeV}$ or higher using innovative cost saving techniques. This is an exciting option and is beginning to receive the careful attention it deserves. The issue before the community will be to weigh the scientific merits against the probable time scale. This will bring in issues of technical, financial and political feasibility. If the desertron physics cannot arrive well before 1995, then the overall scientific needs of the next decade will not be met and the Dedicated Collider is 
the unique facility that can satisfy the need for higher energy. The DC cms, energy is more than $4 \mathrm{TeV}$, and the luminosity will be more than $10^{31} \mathrm{~cm}^{-2} \mathrm{sec}^{-1}$. The natural center of mass energy scale for constituent collisions for this collider is $500 \mathrm{GeV}$ to $1 \mathrm{TeV}$. As discussed in the next chapter, the scientific program in this energy range is extremely rich and the productivity relative to $\mathrm{TeV}$ I promises to be easily a hundredfold.

The situation with regard to lepton-hadron collisions is very much the same as that outlined above for hadron-hadron collisions. Neutrino and muon scattering experiments that are done in the TeV II program will be operating at cms energies of less than $35 \mathrm{GeV}$, at best. The potential physics gains in increasing the available energy by an order of magnitude are enormous, again as discussed in the following chapter. Here again, European intentions are not completely clear, but the prospects for the HERA project seem reasonably good and do have impact on planning in this country.

We shall discuss the role of e-p physics at the DC under two possible scenarios: 1) The HERA project is completed well after 1990,2) HERA is approved immediately and commences doing physics in 1990.

In the absence of HERA, the arguments for the construction of a $10 \times 2000 \mathrm{GeV}^{2}$ e-p facility at the DC are quite compelling and can provide a primary impetus for undertaking the DC project. For a modest investment in a $10-\mathrm{GeV}$ electron ring, the return is a unique facility capable of extending leptgn-nucleon scattering measurements by two orders of magnitude in $Q^{2}$ as well as providing sensitive tests of new phenomena such as quark substructure and right-handed currents. Extension of the facility to $40 \mathrm{GeV}$ on $2 \mathrm{TeV}$ will be a viable future option.

Were HERA to be approved with physics scheduled to commence in 1990, there are two approaches one might take toward the incorporation of an e-p facility at the DC. The first would be to engage in a race with HERA, inasmuch as the energies and luminosities are comparable, with the hope of turning on first. Although one might actually win such a race, the advantages enjoyed by HERA, a greater number of interaction regions and more dedicated e-p running time, would probably eventually win out. The second approach would be to build the 10-GeV electron ring on a time scale comparable with that of HERA but only as a first step to an ultimate 40-GeV elęctron ring. In this scenario, the rapid attainment of $40 \times 2000 \mathrm{GeV}^{2}$ would become the primary goal, although one would initiate studies at $10 \times 2000 \mathrm{GeV}^{2}$, if only to gain early working experience with ep collisions.

Clearly the timing of approval of HERA is crucial to determining which of the scenarios described above is applicable. In any case, we certainly should not now be immobilized by uncertain European plans for its future. 


\section{B. Why an "intermediate range" ring at Fermilab?}

\section{Technical Benefits}

With commissioning of the Saver, Fermilab possesses the highest-energy proton beam in the world, readily available for injection into a new storage ring. Furthermore, the site is large enough for a new ring, and an extensive array of support facilities exists. In particular, the large reservoir of superconducting-system experience at Fermilab is well beyond that of any other laboratory in the world. The magnet factory is already in existence. Thus Fermilab has an especially cost-effective starting position for consideration of any dedicated collider ring.

\section{Collider Benefits}

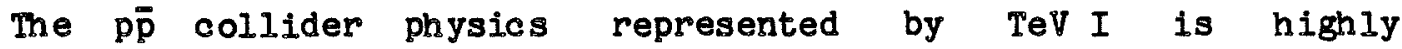
cost-erfective and will be extremely exciting and productive. Nevertheless, the $\mathrm{TeV}$ I collider program is compromised by having to share the Saver with the Tev II program, both in terms of physical space and running time. The existence of at most two collision regions limits the amount and variety of physics that can be explored.

It must be kept in mind that the Saver/TeV II/TeV I program began as an R\&D effort of modest scope and gradually evolved into the present project. The Dedicated Collider will make it possible to separate collider physics from the saver ring, thereby allowing fuller exploitation of the fixed-target program, as well as opening up major new opportunities for study of hadron-hadron collisions at higher energy and luminosity, and with an ep collider facility at modest incremental cost.

\section{Fixed-Target Benefits}

Over the past several years, the fixed-target program at Fermilab has been scheduled to run for less than $50 \%$ of the available time. During this period, the reasons for less than full utilization were related to fiscal constraints as well as the Saver/TeV I/TeV II construction schedule. Nonetheless, this experience has taught us that the accelerator systems, beam line systems, and large complicated detectors require substantial periods of turn-on and debugging time before reliable results can be obtained. In short, the end effects for brief runs ( $\$ 4-6$ months) are substantial.

If it were possible to schedule the experimental areas for $\geq 90 \%$ utilization, at least three important benefits could be realized:

i) End effects would be minimized. 
ii) More time could be allocated to the debugging and calibration of large complicated detectors. More precise and better-understood results would be forthcoming. Presently, experiments often enter the data-taking mode with less than optimal understanding of the detector and runs are cut short to accommodate additional experiments, whose goals may also be compromised because of limited running time. In addition, experimentation in this field, as in others, requires the flexibility to learn by experience and then to return with improvements. This process is often short-circuited by the pressures of limited running time.

iii) Finally, one often hears of the reduced number of "spigots" at American high-energy accelerators. What is really at issue is the total number of secondary beam hours available to experimenters. One can increase this number either by building more beams or by running those we have longer. The combination of the Dedicated Collider and the $\mathrm{TeV}$ II project presently under way allows Fermilab to make significant progress in both areas.

In summary, the benefits to the fixed target program are substantially more than a simple factor of two in utilization.

\section{Why Use "Conventional" Superconducting Technology?}

The dedicated $\mathrm{p} \overline{\mathrm{p}} / \mathrm{ep}$ collider is designed to use "conventional" state-of-the-art technology as much as possible. For example, the magnets and refrigeration system bear great similarity to those used in the Saver. The advantage to using this technology is that it is proven. For more than 10 years, high-energy physics has made a major, painful R\&D investment in superconducting technology. The Dedicated Collider will capitalize on it now rather than invest several more R\&D years before building the next generation of colliders. It has been estimated at Snowmass and at the recent Cornell 20-TeV workshop that about four years of R\&D will be required before production can start on a magnet of substantially higher magnetic field.

\section{Why ep/pp Collisions?}

Possible alternative Fermilab dedicated colliders might be either $\mathrm{e}^{+} \mathrm{e}^{-}$or pp. The LEP and SLC projects already represent a very large investment in $\mathrm{e}^{+} \mathrm{e}^{-}$collider physics in the $100-\mathrm{GeV}$ center-of-mass energy range. This alone raises a potent argument against another new $e^{+} e^{-}$ commitment at Fermilab, unless the physics output were extraordinarily rich and the facility could produce much higher luminosity at a reasonable cost.

A pp collider somewhat above $1 \mathrm{TeV}$ per beam is also a possible alternative. Here the increase in luminosity compensates in many ways 
for the lower energy. There are two arguments agajnst this alternative. One is that a luminosity in excess of $10^{32} \mathrm{~cm}^{-2} \mathrm{sec}^{-1}$ is required to match the hard-collision physics qutput of the p $\bar{p}$ collider (assuming for it a luminosity of 1 to $4 \times 10^{31} \mathrm{~cm}^{-2} \mathrm{sec}^{-1}$ ). But at such luminosities basic limitations on detector capabilities become severe and the effective utilization of the high luminosity is cast into doubt. Another argument for a large, roughly site-filling $p \bar{p}$ ring over a smaller pp ring is growth potential. In the long run, one can consider upgrades of the hadron-hadron collider in either energy (if 8-12T magnet technology bears fruit) or luminosity (by adding a second proton ring). In addition, one may choose a major upgrade of ep physics by adding a large $30-50 \mathrm{GeV}$ concentric electron ring. There is a clear advantage in having services, collision halls, etc. optimally located from the start.

\section{E. Is there Scientific Justification?}

The foremost reason for embarking on such a project must be the physics justification. This will be discussed in more detail in the next chapter. While a factor of two in cms energy more than TeV I may seem not large, it must be kept in mind that this factor of two coupled with the higher luminosity and more available beam time allows exploration of hard collision subprocesses in the $500 \mathrm{GeV}-1 \mathrm{TeV}$ range of subenergies. This regime is one of special theoretical interest, especially with respect to the structure of electroweak interactions. Fig. I-2 exhibits the capabilities of various facilities to explore hard collision processes. It is clear that this dedicated collider represents a major step beyond $\mathrm{TeV} 1$. In addition, the collider would, until the Soviet UNK turned on, or until the LEP tunnel were filled with a proton ring, far surpass in energy and subenergy any facility worldwide.

No one has yet built an ep colliding-beam facility. ep collisions, which have yielded so much insight into hadron structure in the past, have no less promise for the future. Even setting aside production of new states in ep collisions, the study of "conventional" phenomena such as QCD jets or weak-interaction form factors should be especially fruitful. ep collisions share many of the features of simplicity possessed by $e^{+} e^{-}$collisions, as well as having some of the richness and higher energy of the phenomena seen in hadron-hadron colliders. If the $p \bar{p}$ phenomena differ in any essential way from $e^{+} e^{-}$collider phenomena, then it is important to have means of interpolating between the extremes. ep collisions provide that interpolation. 

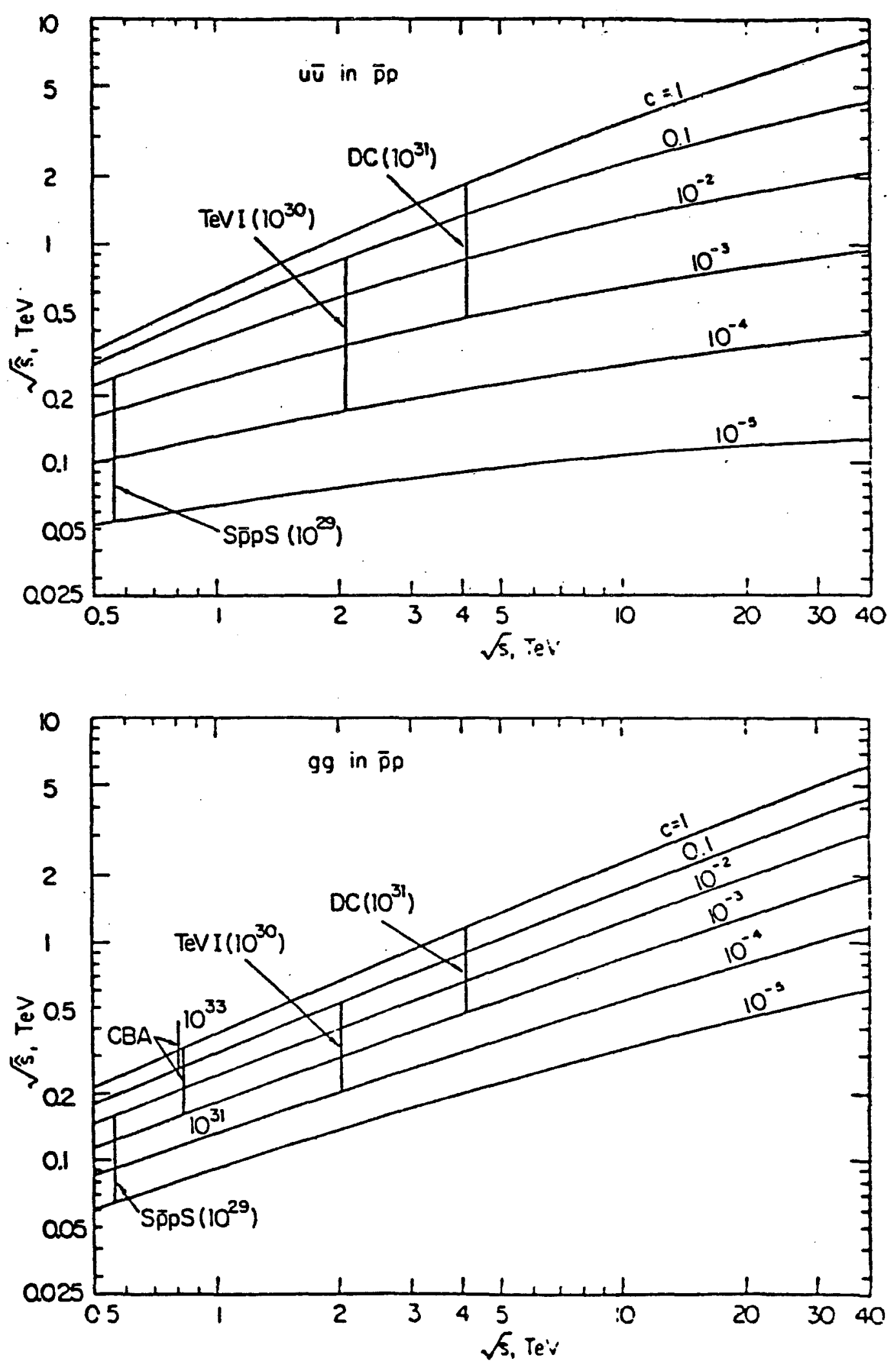

Fig. I-2. Atta1nable Hard-Colliston Invariant Mass as a function of Collider Invariant Mass. (a) The ū subprocess for $\bar{p} p$ coll1ders.

(b) the $g 8$ subprocess for pp and pp colliders, with allowance for the different luminosities of the various facllities. $\sigma=c / \hat{s}$ 


\section{PHYSICS OPPORTUNITIES}

The conventional possibilities for collider physics have been spelled out in many places, most recently in the 1982 Snowmass Workshop. Although these are unlikely to represent the most far-reaching results to be obtained with the Dedicated Collider, they provide useful benchmarks for assessing the sensitivity and richness of a new facility. In this - brief chapter, we show how the Dedicated Collider responds to the expected physics. We also show how the DC opens a new and important energy regime, and indicate the unique potential of this device for dramatic experimental progress.

\section{A. Beyond the Bellwethers}

The spectacular results from sp̄ps experiments UA1 and UA2 are important not only for their direct contributions to science, but also for what they portend for future facilities. They demonstrate that:

1. $\overline{\mathrm{pp}}$ colliders work. Intense antiproton sources of good emittance can be bujit. The beam beam interactions are (at worst) no more disruptive than anticipated.

2. Hard collisions occur in hadron-hadron interactions. The theoretical rate projections have been reasonably accurate, and extraction of signals from background has not been any more difficult than generally anticipated. Indeed, those who felt that a very high integrated luminosity would be required to extract the signal for the leptonic decay of the were unprepared for the convincing evidence for the intermediate boson that emerged from only a few events.

3. Multijet events are manifestly analyzable. The striking LEGO plots of these events promise a rich future for fine-grained calorimetric techniques for identifying and measuring electromagnetic and hadronic jets. One can well imagine the measurement of inclusive spectra of jets and leptons becoming relatively pedestrian as techniques of multijet spectroscopy mature. We may already anticipate relatively strong statements from the next spps running period on the existence of the top (or other) quark(s) in the mass range of $20-60 \mathrm{GeV} / \mathrm{c}$, based on the measurement of two or more particles (or jets) per event. These multijet phenomena are likely to dominate experimental and theoretical attention within the next few years, and certainly in the time frame we consider here for the DC. The mastery of multijet spectroscopy will liberate us from reliance upon low-branching-ratio signatures for interesting new phenomena. One must also bear in mind that the c.m. energy of the DC is more than seven times greater and the luminosity more than 200 times greater than what is now available at the sp̄ps.

An important element of the Dedicated Collider complex is a high-luminosity electron-proton collider. Initially projected for 10 
GeV $\times 2 \mathrm{TeV}$ operation, this facility has significant growth potential, since a $40 \mathrm{GeV}$ electron ring can be accommodated in the DC tunnel. In the initial phase, the $D C$ electron option is competitive with the proposed HERA machine. In its second stage, the DC ep collider is unique in the world.

In what follows we survey the physics opportunities presented by the Dedicated Collider. We proceed from the conventional features implied by the standard model to more speculative topics, and close the $\mathrm{p} \overline{\mathrm{p}}$ discussion with a very brief general discussion of the rates for hard collisions among partons, along with a discussion of the limitations of the calculations.

We then turn to an outline of the DC's capabilities for electron-proton physics. Again this topic has been the subject of many proposals and summer studies, so we shall content ourselves with a discussion of the main points. Although an ep collider has not been built before, the virtues of ep collisions are well appreciated. The electron provides a well understood and apparently structureless probe, and the ep facility nicely complements the existing and projected $\mathrm{e}^{+} e^{-}$ and hadron-hadron (i.e., quark-quark) colliders. ep collisions are particularly sensitive to deviations from the standard model; right-handed currents, quark substructure, lepton-quark compositeness contact term, and clean signatures for heavy quark (or leptoquark) production. These opportunities for discovery are examined in section $G$ of this chapter.

\section{B. Electroweak Phenomena}

The discovery of the intermediate boson establishes, as expected, the $100 \mathrm{GeV}$ regime of c.m. energy as the natural scale of the electroweak interactions. The sp̄ps and Tev I programs, and especially the LEP and SLC electron-positron colliders, should provide a rather tilorough exploration of this energy regime. In addition, TeV I will make possible the first look beyond this energy scale. As already mentioned, the natural habitat of the $D C$ is in the realm of hard-collision invariant masses between 0.5 and $1 \mathrm{TeV}$. This is not to say that conventional electroweak/QCD physics will be neglected, however.

As an example, we show in Table II-1 the number of standard model intermediate bosons to be expected in a standard run (integrated luminosity $\int \mathrm{Xdt}=10^{38} \mathrm{~cm}^{-2}$ ) at the DC. The expected number is of order $10^{6}$, which should be large enough to permit many detailed studies. This represents a significant increase over the rates anticipated for the SppS and $T e V I$, and is also competitive with what might be ach jeved in a bigh-luminosity CBA. For the neutral gauge bosons $z^{0}$ the $e^{+} e^{-}$ colliders would seem to retain a decided advantage in event rate.

The situation is similar for the production of pairs of gauge bosons, a measurement which provides some of the motivation for LEP II. 
The cross section is sensitive to three-gauge-boson couplings, and has been advocated as a test of the non-Abelian nature of the interaction. Whether it will in fact be the most sensitive test remains to be seen. In any case, as shown in Table II-2, the DC will produce these events in interesting numbers. The cross section for the related Wr final state, which is sensitive to the magnetic moment of the intermediate boson, is strongly dependent on the cut imposed upon the photon momentum. The anticipated event rate is typically greater than or equal to the pair production rates in Table II-2.

Within the standard model, the spontaneous symmetry breaking is accomplished by an elementary scalar Higgs boson. The mass of the Higgs boson is an arbitrary parameter of the theory as it is currenty understood, subject only to the bounds

$$
7.4 \mathrm{GeV} / \mathrm{c}^{2}<\mathrm{M}_{\mathrm{H}} \approx 1 \mathrm{TeV} / \mathrm{c}^{2} \text {. }
$$

It is plausible that the upper bound, which is based on the consistency of perturbation theory, can be improved to approximately $400 \mathrm{GeV} / \mathrm{c}^{2}$. If $M_{H} \leqslant 40 \mathrm{GeV} / \mathrm{c}^{2}$, it should be possible to detect the Higgs boson in $\mathrm{Z}^{\circ}$ decays at SLC or LEP. LEP II could perhaps extend the limit to about $100 \mathrm{GeV} / \mathrm{c}^{2}$ in the process

$$
\mathrm{e}^{+} \mathrm{e}^{-}+\mathrm{H}+\mathrm{Z}^{0}
$$

The DC is sensitive to still higher masses. The production mechanism of $8 \mathrm{~g} \nrightarrow \mathrm{H}$ via a fermion triangle is sensitive to the mass of the top quark, as shown in Fig. II-1. Table II-3 shows the highest Higgs boson mass for which 100 events will be produced for the benchmark luminosities. For Higgs masses in excess of about $2 M$, the dominant decay mode will be into pairs of gauge bosons. This would provide a characteristic signature for hadron-jet spectroscopy. The mass range $M_{W}<M_{H_{1}}<2 M_{W}$ is
more problematic, and may require good luck-or an $e^{+} e^{-}$collider.

Should the top quark be very heavy, or should a fourth fermion generation exist, it is of interest to search for heavy quarks using the methods now being evolved at the sp̄ps. Pair production cross sections estimated from the gluon fusion mechanism are shown in Fig. II-2. The DC again considerably extends the range of accessible masses, as summarized in Table II-4.

A simple extension of the standard model would entail the existence of additional gauge bosons. In the case of a right-handed $\mathrm{W}$-boson, which would restore left-right symmetry at high energies, the ep facility of the Dedicated Collider would be an important diagnostic tool. Couplings of additional gauge bosons to the i.ight fermions are evidently model dependent, but reasonable cross section estimates for production in p $\bar{p}$ collisions may be had by assuming universality of the 

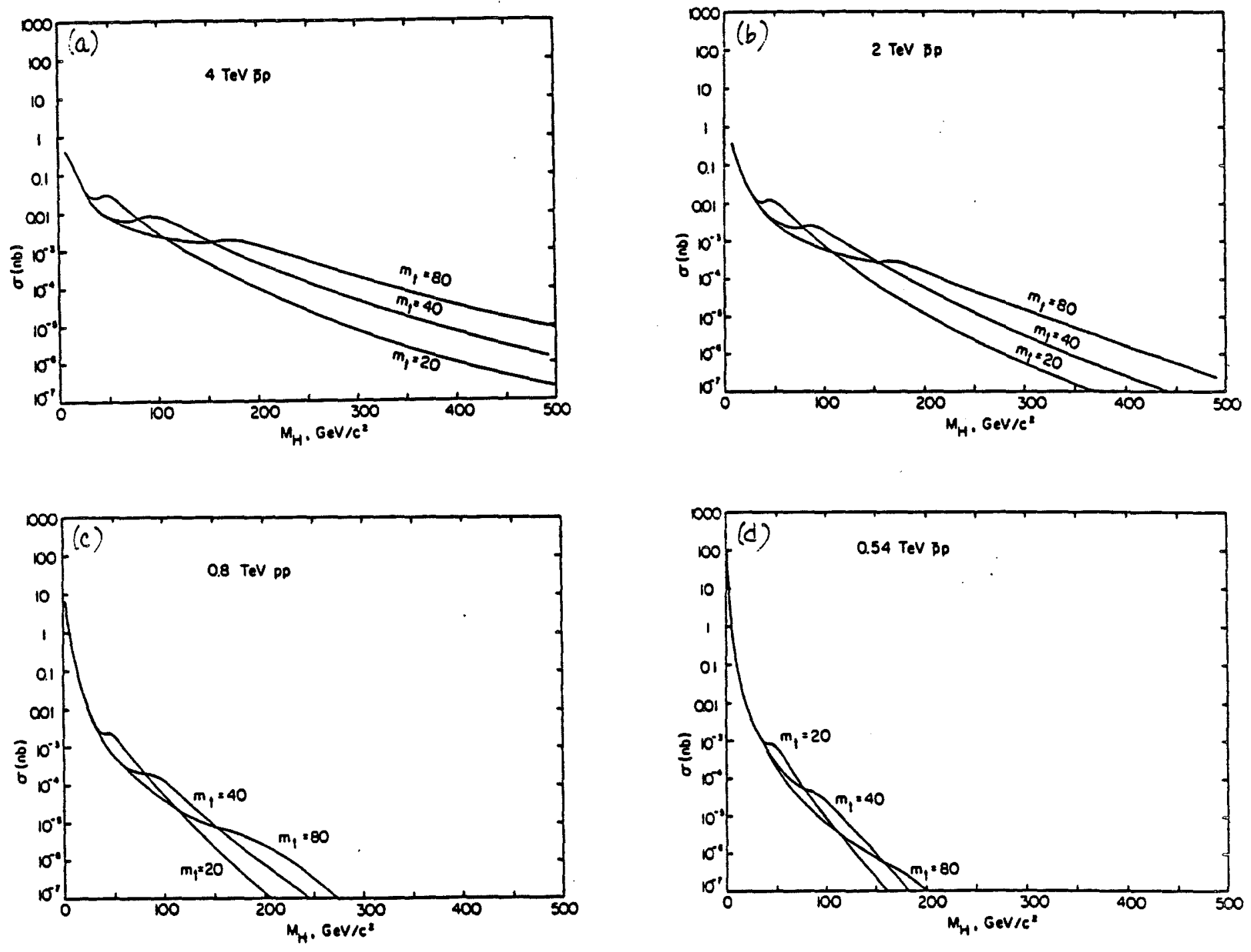

Eig. II-1. Cross sections for production of Higgs bosons in hadron-hadron collisions. (a) $4 \mathrm{TeV}$ pp; (b) $2 \mathrm{TeV} \overline{\mathrm{pp}}$; (c) $0.8 \mathrm{TeV} \mathrm{pp}$; (d) $0.54 \mathrm{TeV}$ pp. 


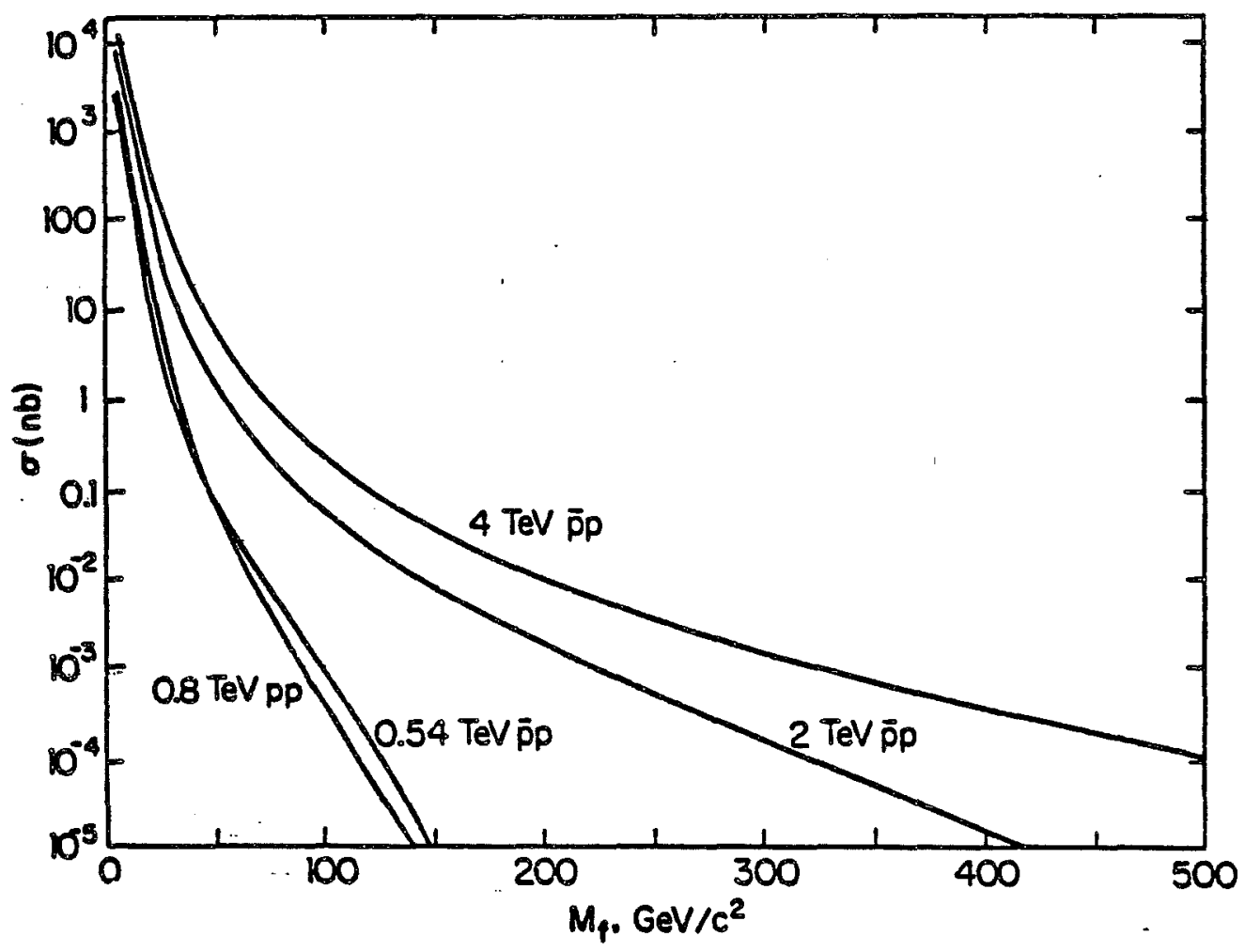

F18. II-2. Cross sections for production of heavy quark pairs in hadron-hadron collisions. 



Fig. II-3. Cross sections for production of heavy electroweak gauge bosons in hadron-hadron collisions. (a) $4 \mathrm{TeV}$ pp; (b) $2 \mathrm{TeV}$ pp; (c) $0.8 \mathrm{TeV} \mathrm{pp}$; (d) $0.54 \mathrm{TeV} \overline{\mathrm{pp}}$. 
gauge couplings. Cross sections computed on this basis are displayed in Fig. II-3. The highest masses for which 100 events are produced are tabulated in Table II-5. The DC sensitivity extends well beyond $1 \mathrm{TeV} / \mathrm{C}^{2}$.

In the context of the DC scale of subenergies, dilepton production does not appear especially interesting for its own sake. Nevertheless, as Fig. II 4 shows, there is sensitivity at the level of one event per $\mathrm{GeV} / \mathrm{c}^{2}$ in $\mathrm{do} / \mathrm{dM}$ out to an invariant mass of approximately $250 \mathrm{GeV} / \mathrm{c}^{2}$.

\section{Hadron Jets}

Early running at the CERN SpppS has confirmed the expectation that the cross sections for hard scattering of constituents are large. Moreover, LEGO displays of the kind included as Fig. II-5 have shown that for an important class of events the jets are well collimated, isolated, and straightforward to analyze. Already in limited running, hard collisions have been observed at c.m. energies in excess of those that may be attained in $\mathrm{e}^{+} \mathrm{e}^{-}$collisions for a decade or more.

Jet studies in hadron-hadron collisions have traditionally been viewed as less incisive than those carried out in electron-positron annihilations or in lepton-nucleon scattering because of the added complexity of events. The Spps experience indicates that, as hoped, the hard scattering events take on a much simpler aspect at high energies, and there is no impediment to detailed analyses. We may therefore expect to take advantage of the higher energies attainable in hadron-hadron collisions and of the greater diversity of elementary interactions made possible by our unseparated broad-band parton beams.

To give an indication of the expected cross sections, we show in Fig. II-6 the lowest-order QCD hard-scattering contributions to $d \sigma / d y d p_{T}$ at $90^{\circ}$ in the c.m. Our current understanding of QCD seems not to justify a more elaborate calculation. In any event, the prediction for $\checkmark / s=0.54 \mathrm{TeV}$ is in reasonable agreement with the preilininary data from the UA 1 experiment. One may read of $f$, as a figure of nerit, the maximum (single-jet) transverse momentum for which a standard run will yield 100 events per bin of 1 unit of rapidity and $1 \mathrm{GeV} / \mathrm{c}$ of transverse monentum, i.e., the point at which

$$
\left.\int d t \mathcal{L} \cdot \frac{d^{2} \sigma}{d y d p}\right|_{y=0}=100 /(\mathrm{GeV} / \mathrm{c}) .
$$

These values of $p_{\mathrm{T}}$ are collected in Tabie II- 6 . At the Dedicated Collider, one can anticipate extensive studies of hadron jet phenomena for jet transverse momenta in excess of $350 \mathrm{GeV} / \mathrm{c}$, corresponding to elementary collisions at $\sqrt{\mathrm{s}} \sim 700 \mathrm{GeV}$. Exploratory studies will be possible to considerably higher transverse momenta. 


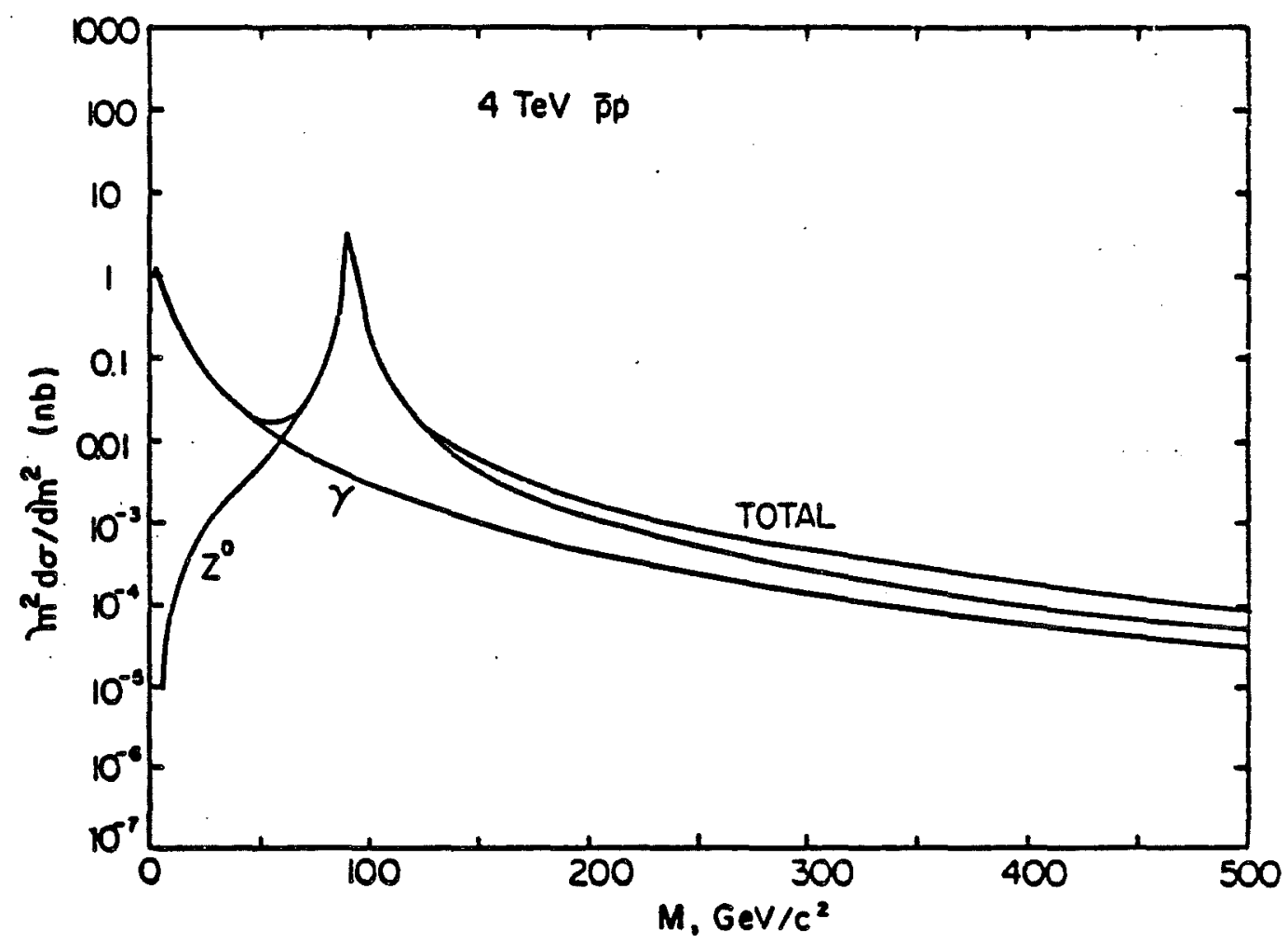

F1g. II-4. Differential cross section $\cdot \mathrm{m}^{2} \mathrm{d \sigma} / \mathrm{dm}^{2}$ for production of massive lepton pairs in $4 \mathrm{TeV}$ pp collisions.

TRANSVERSE ENERGY DEPOSITION

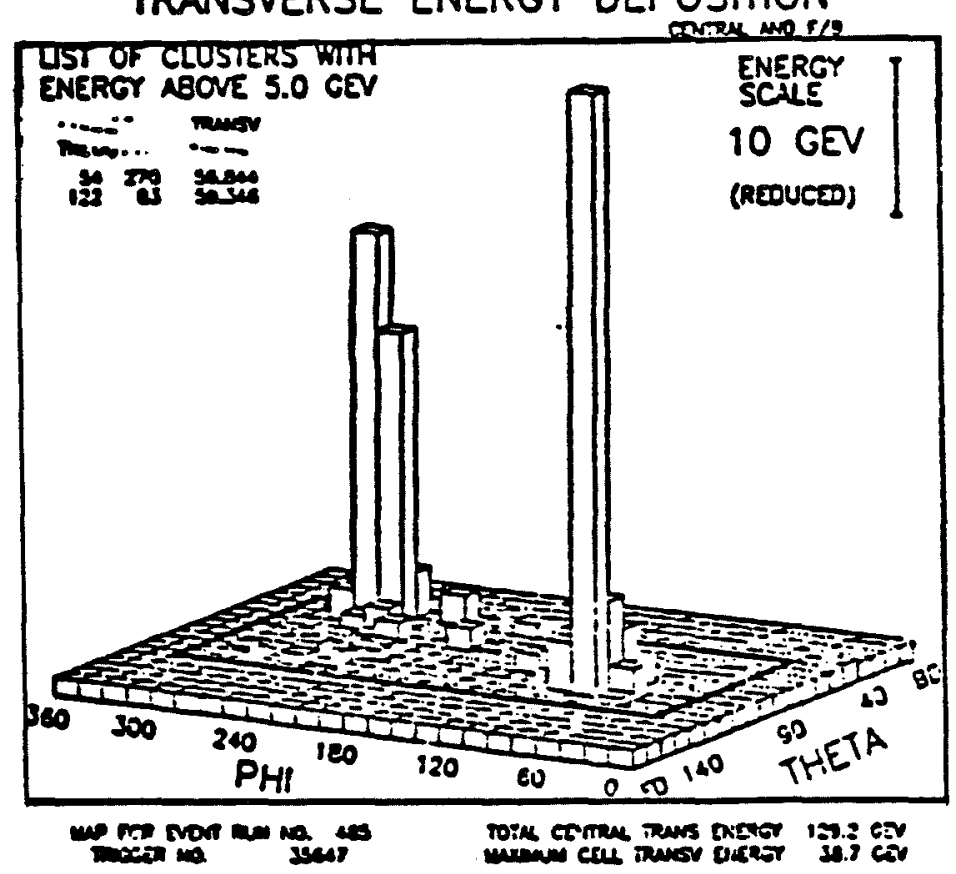

F18. II-5. "LEGO" display of a typical two-jet event observed in the UA2 detector at the Spps. 


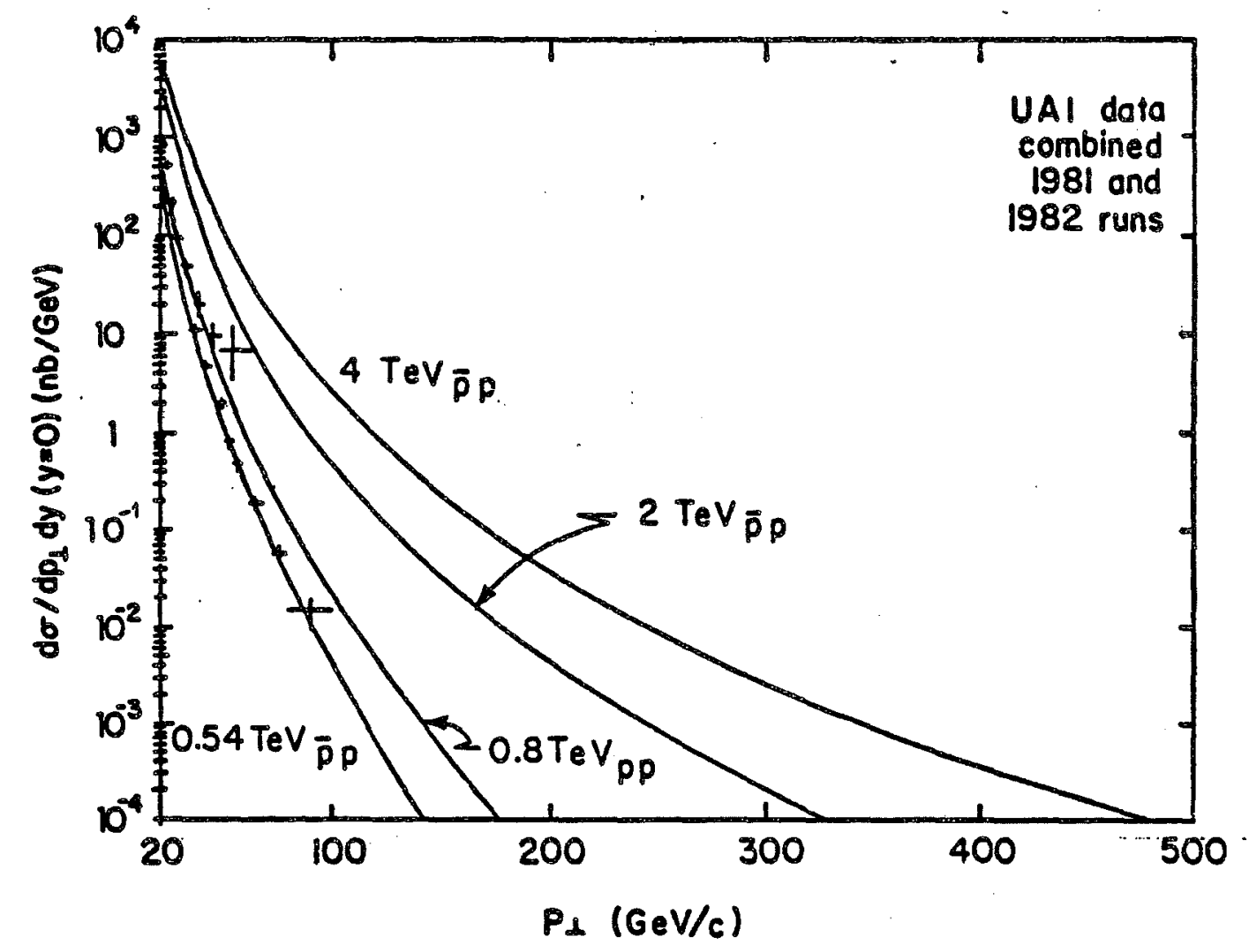

F18. II-6. Hard scattering contribution to the production of hadron jets at $90^{\circ}$ in the c.m. The 1981-1982 UA1 data collected in $0.54 \mathrm{geV}$ pp collisions are shown for reference.

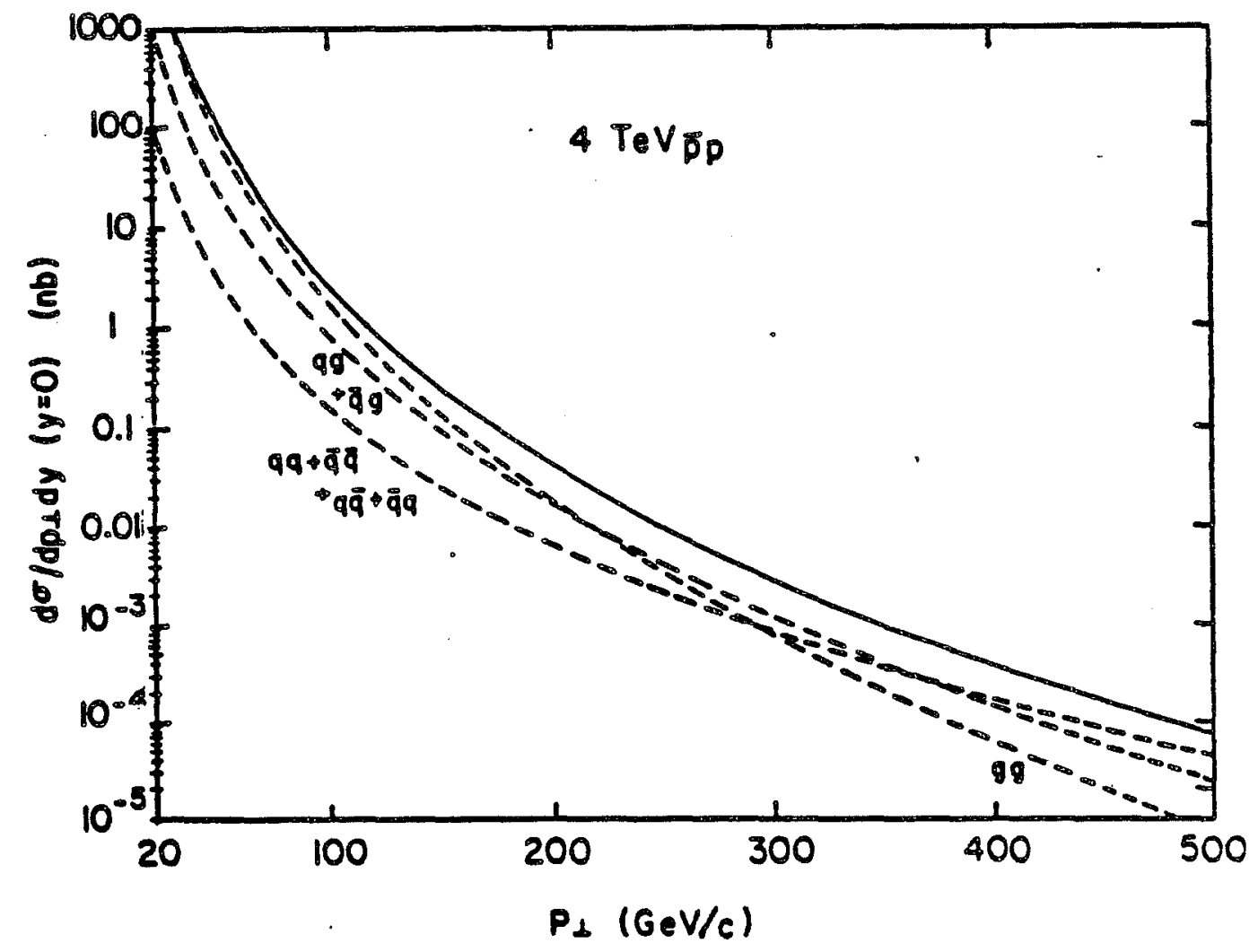

F18. II-7. Jet production by components in $4 \mathrm{TeV}$ pp collisions. 
The prospect of studying fully-developed jets is enhanced by the possibility of distinguishing between gluon jets and quark jets by kinematic selections. How this might be done is indicated first in Fig. II-7, which shows separately the contributions to $d^{2} \sigma / d y d p_{T}$ of the

$$
\begin{aligned}
& g g \rightarrow(g g \text { and } q \bar{q}) \\
& g q \rightarrow g q \text { and } g \bar{q} \rightarrow g \bar{q} \\
& \left\{\begin{array}{l}
q q \rightarrow q q \\
\overline{q q} \rightarrow \overline{q q} \\
q \bar{q} \rightarrow q \bar{q}
\end{array}\right.
\end{aligned}
$$

processes. Because the cross section for $g g \rightarrow q \bar{q}$ is negligible compared to that for $g g+g g$, these three classes of processes correspond closely to two-gluon, (anti)quark-gluon, and two (anti)quark jets. At modest transverse momenta, of order $100 \mathrm{GeV} / \mathrm{c}$ or less, the two-gluon final state is dominant. The mix changes markedly at larger values of $p_{T}$, so that quark jets ultimately prevail.

Another method of separation is made possible by the different rapidity dependence of the components. Figures II-8 (a) $-(e)$ show the behavior of

$$
\frac{d \sigma}{d p_{T} d y_{1} d y_{2}} \mid y_{2}=-y_{1}
$$

as a function of $y$ for various fixed values of $p_{T}$. The gluon-gluon process prevails at small $\mathrm{p}_{\mathrm{T}}$ and small rapidities, whife the reactions involving valence quarks become dominant at large $p_{T}$ and large rapidities. A similar effect is shown in Figs. II-9 (a) $-(c)$, where the transverse momentum of the jet is held fixed at $\mathrm{p}_{\mathrm{T}}=200 \mathrm{GeV} / \mathrm{c}$, and the c.m. rapidity $y_{B}=y_{+}+y$ of the colliding partons is varied. The possibilities for dramatic changes in the mix of jets are readily apparent.

Interesting as the study of two-jet events may now seem, it may well be rather straightforward and thus rapidly assume the traditional role of thabha scattering in $\mathrm{e}^{+} \mathrm{e}^{-}$colliders: prominent, quickly and accurately measurable, and thereafter neglected by all but the Feynman-diagram computer technologists. Multijet events and multijet spectroscopy. would then become the focus of research interests for perturbative QCD in this regime. Again, it is the high energy, diversity of processes, and simplicity of jet spectroseopy which raise our hopes. 



F18. II-8. Contributions of hard-scattering components to symetric $\left(y_{1}+y_{2}=0\right)$ jet production, for specified transverse momentum of each jet in $4 \mathrm{TeV}$ pp collisions. (a) $p_{\perp}=50$ GeV/c; (b) $p_{\perp}=100 \mathrm{GeV} / \mathrm{c}$; (c) $p_{\perp}=200 \mathrm{GeV} / \mathrm{c}$; (d) $p_{\perp}=$ $300 \mathrm{GeV} / \mathrm{C} ;(e) P_{\perp}=400 \mathrm{GeV} / \mathrm{c}$. 



Fig. II-9. Contributions of hard-scattering components to jets of $p_{1}=200 \mathrm{GeV} / \mathrm{c}$, as a runction of the rapidity $y_{1}+y_{2}$ of the hard-collision c.m. (a) $y_{1}+y_{2}=0$; (b) $y_{1}+y_{2}=1$; (c) $y_{1}+y_{2}=2$. 


\section{New Landmarks in 1-TeV Pnysics}

In many ways, the scale of $1 \mathrm{TeV}$ represents the frontier of our ignorance. It describes a regime in which we have, as yet, no direct experimental information and one in which our current understanding seems to compel the existence of new phenomena. The necessity of new physics is more convincing than the argument for any specific manifestation of the new scale, so it is important to explore this region with good sensitivity to many possibilities.

What are the physics landmarks in this regime? The clearest one is given by the fundamental parameter of electroweak spontaneous synmetry breaking, the vacuum expectation value $\langle\phi\rangle_{0}$ of the Higgs field, which is equal to about $174 \mathrm{GeV}$. The origin of this scale, which is considerably larger than that of the intermediate bosons, is dimly understood. This alone provides a solid stimulus to the exploration of this regime. Most lines of theoretical speculation have as a primary goil the improved understanding of the dynamics of the Higgs sector. Typiuti sonarios populate the region between $100 \mathrm{GeV}$ and $10 \mathrm{TeV}$ with a multitude of new particles. In each of the proposed scenarios:

\section{Technicolor \\ Supersymmetry \\ Compositeness}

there are clearly identiflable signals of this dynamios at energies at or below $G_{F}-172 \simeq 250 \mathrm{GeV}$. Each of these alternatives has a light sector of bosons (and/or fermions) whose existence is associated in a fundamental way to the approximate chiral symmetries at the new interaction scale. This light sector comprises the set of least massive members of the family of new particles and is a general feature of any dynamics at this scale.

In technicolor the light sector is bosonic--the specific new particles have been called technipions--while in the supersymmetric models both bosons (squarks and sleptons) and fermions (gluino, photino,...) residt. In composite models the role of the light sector of the theory is provided by some (or all) of the ordinary quarks and leptons. Here, therefore, the signal of the new dynamics will show up directly in the hard scattering of quarks and leptons. We will discuss each of these alternatives in turn.

\section{Technicolor}

In the standard electroweak theory, the spontaneous symmetry breaking is accomplished by the action of a complex doublet of elementary scalar fields. Subject to constraints imposed by neutral current phenomenology, there may in prinoipi.t be any number of elementary scalars and the resulting Higgs bosons. How many there are, what are the masses of the surviving physical scalinom, and what are their couplings to ordinary matter can only be settled experimentally. The standard theoretical framework offers no guidance, otier than rather broad (and nonrigorous) bounds on the Higgs boson mass. 
An alternative description goes by the name of dynamical symmetry breaking or, colloquially, technicolor. This program attempts to find a dynamical basis for the Higgs scale in terms of new strong (technicolor) interactions at a scale of about $1 \mathrm{TeV}$, and thus to explain the breakdown of $\mathrm{SU}(2), \mathrm{U}(1)_{\mathrm{Y}} \rightarrow \mathrm{U}(1)_{\mathrm{FM}}$ and the generation of fermion masses. In this approach, spinless bound states of heavy fermion-antifermion pairs play the role of the elementary scalars of the standard model. The lightest of these, dubbed technipions, are the most immediately accessible to experiment.

No phenomenologically acceptable model of dynamical symmetry breaking has been developed, and so many detalls of the conjectured spectrum are unsettled. The general idea of dynamical symmetry breaking is however so natural and appealing, and the general arguments for structure in the few-hundred GeV regime so compelling, that a careful search is mandatory.

A number of the conjectured composite scalar mesons have appreciable couplings to gluons and hence can be produced copiously in hadron-hadron colliders. The so-called technieta $\left(\eta_{T}\right.$ or $\left.P_{8}\right)$ can be produced in two-gluon fusion with the cross sections shown in Fig. II-10. This leads to a sensitivity at the 100 events per run level as shown in Table II-7. For the Dedicated Collider, the maximum accessible mass of $640 \mathrm{GeV} / \mathrm{c}^{2}$ is well above the "expected" mass of $-240 \mathrm{GeV} / \mathrm{c}^{2}$. Heavy pairs of colored technihadrons can also be produced, as indicated in Fig. II-11 with (solid curves) and without (dashed curves) the expected technivector meson enhancements. The maximum accessible masses at the DC as shown in Table II-8 are again well above the conjectured values $M\left(P_{3}\right) \sim 160 \mathrm{GeV} / \mathrm{c}^{2}, \mathrm{M}\left(\mathrm{Pg}_{8}\right)-240 \mathrm{GeV} / \mathrm{c}^{2}, \mathrm{M}\left(\mathrm{P}_{6}\right)$ $-260 \mathrm{GeV} / \mathrm{c}^{2}$. The technihadrons should have distinctive decay signatures involving multiple jets and leptons.

\section{Supersymmetric Partners of the Known Particles}

A possible sign of the incompleteness of the standard model is the arbitrariness that remains even after a minimal unification of the strong, weak, and electromagnetic interactions. The gauge bosons may be said to be prescribed by the local gauge symmetry, but the elementary fermions are put in by hand, and the elementary scalar fields and their self-interactions are, for now, total invention. The possibility of relating vector, spinor, and scalar particles in a way that reduces or eliminates the unwanted freedom of the model has an obvious appeal. The fermion-boson symmetry known as supersymmetry raises the hope that such a simplification might be achieved. However, it is now apparent that the observed particles cannot be supersymmetric partners of each other. Therefore, if supersymmetry is useful on the.present energy scale, it implies a doubling of the spectrum with the following minimal complement of new objects: 

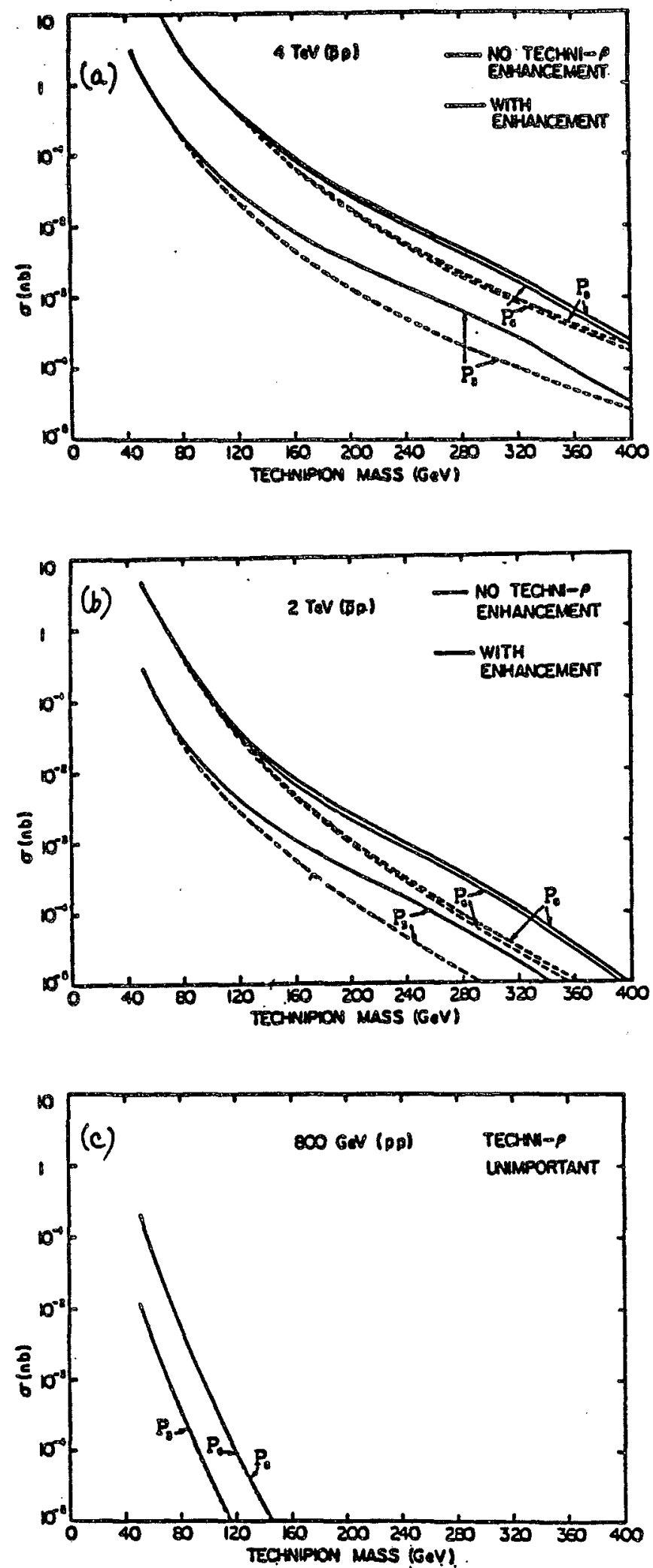

Fig. II-10. Cross sections for pair production of technipions with (solid curves) and without (dashed curves). technirho enhancement. (a) $4 \mathrm{TeV} \overline{\mathrm{pp}}$; (b) $2 \mathrm{TeV} \overline{\mathrm{pp}}$; (c) $0.8 \cdot \mathrm{TeV} \mathrm{pp}$. 


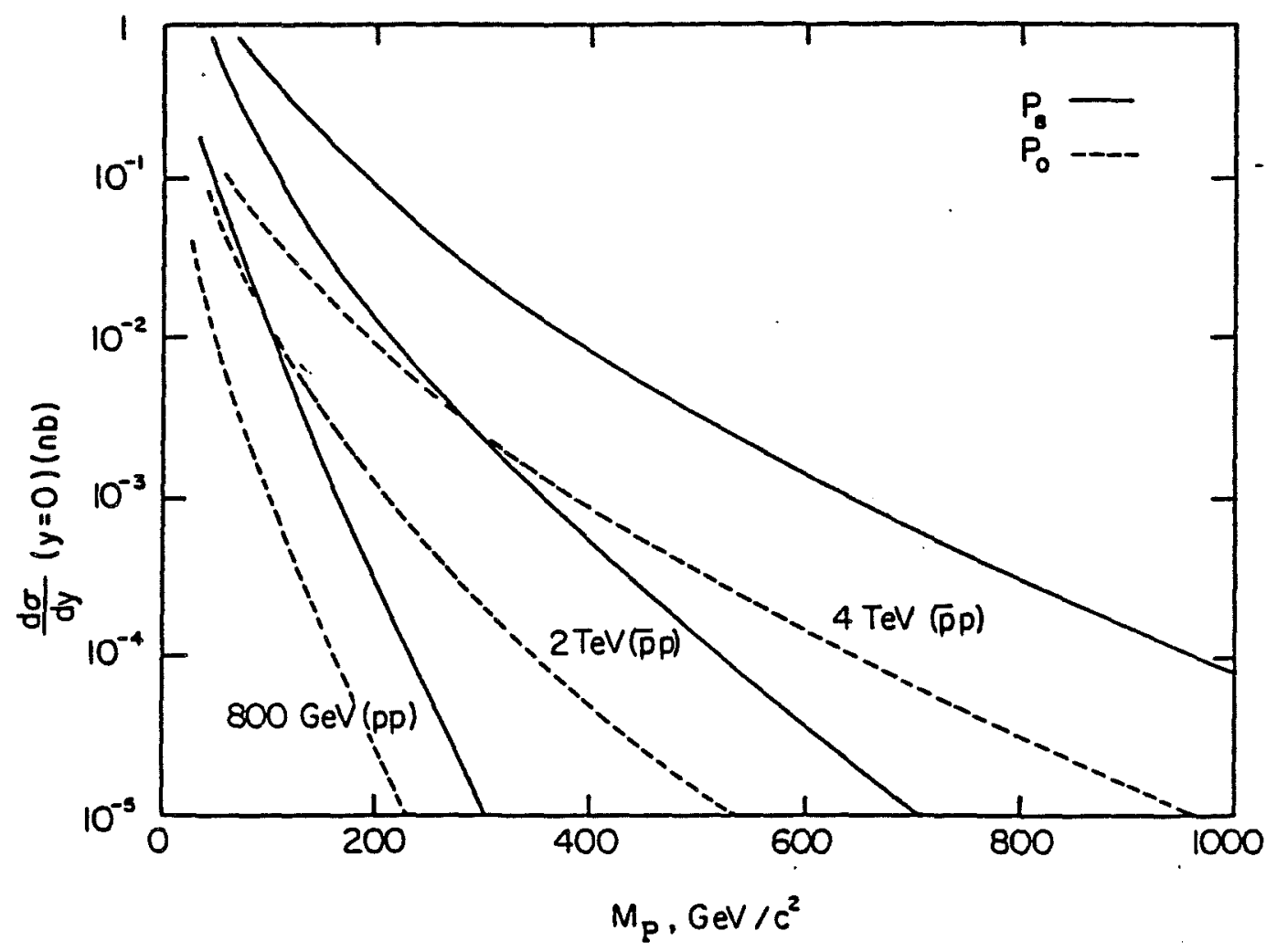

Fig. II-11. Differential cross sections $d \sigma / d y(y=0)$ for production of Higgs-like scalars in hadron-hadron collisions. 
Established Particles

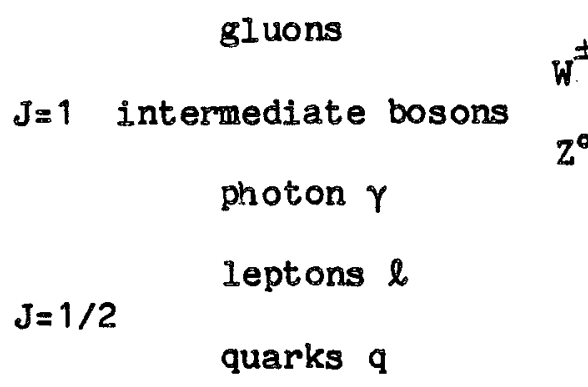

$\mathrm{J}=0 \quad$ Higgs boson $\mathrm{H}$
Supersymatric Partners

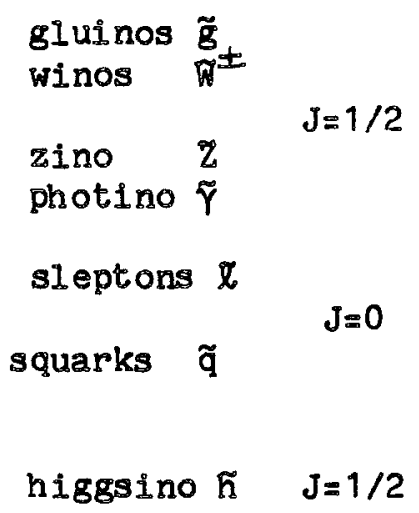

These are plainly not degenerate with the estabilisined particles, so supersymmetry must be broken. No convincing model of broken supersymmetry which meets phenomenological requirements has yet been formulated. Consequently the pattern of masses and decay chains of the superparticles is open to speculation. In contrast, the elementary couplings involving superparticles should be related to known couplings by Clebsch-Gordan coefficients.

While worthwhile in its own right, a complete survey of possibilities would be out of proportion to the importance of superparticles to the justification of the DC. The particles which seem to be of greatest interest for a high-energy hadron collider are the colored squarks and gluinos. The cross section for pair production of squarks is shown in Fig. II-12 as a function of squark mass. iJnder the running conditions anticipated for the $D C$, the event rate is ample in the $100 \mathrm{GeV} / \mathrm{c}^{2}$ regime. As Table II-9 shows, the maximum squark mass for production of 100 pairs is approximately $215 \mathrm{GeV} / \mathrm{c}^{2}$, which exceeds what may be anticipated for other machines.

The expected gluino production rates are still larger, because of tile larger color charge of the gluino. These are shown in Fig. II-13 and Table II-10. In this case, the DC should provide sensitivity out to a mass or $400 \mathrm{GeV} / \mathrm{s}^{2}$.

\section{Compositeness}

The standard model is based upon the notion that the quarks and leptons are elementary particles, and indeed there is direct experimental evidence that they are structureless on a scale of $\sim 10^{-16} \mathrm{~cm}$. However, both nistory and the proliferation of flavors encourage us to consider the possibility that quarks and leptons are themselves composite. The right such model might then predict the spectrum and reduce the arbitrariness inherent in the standard model. 


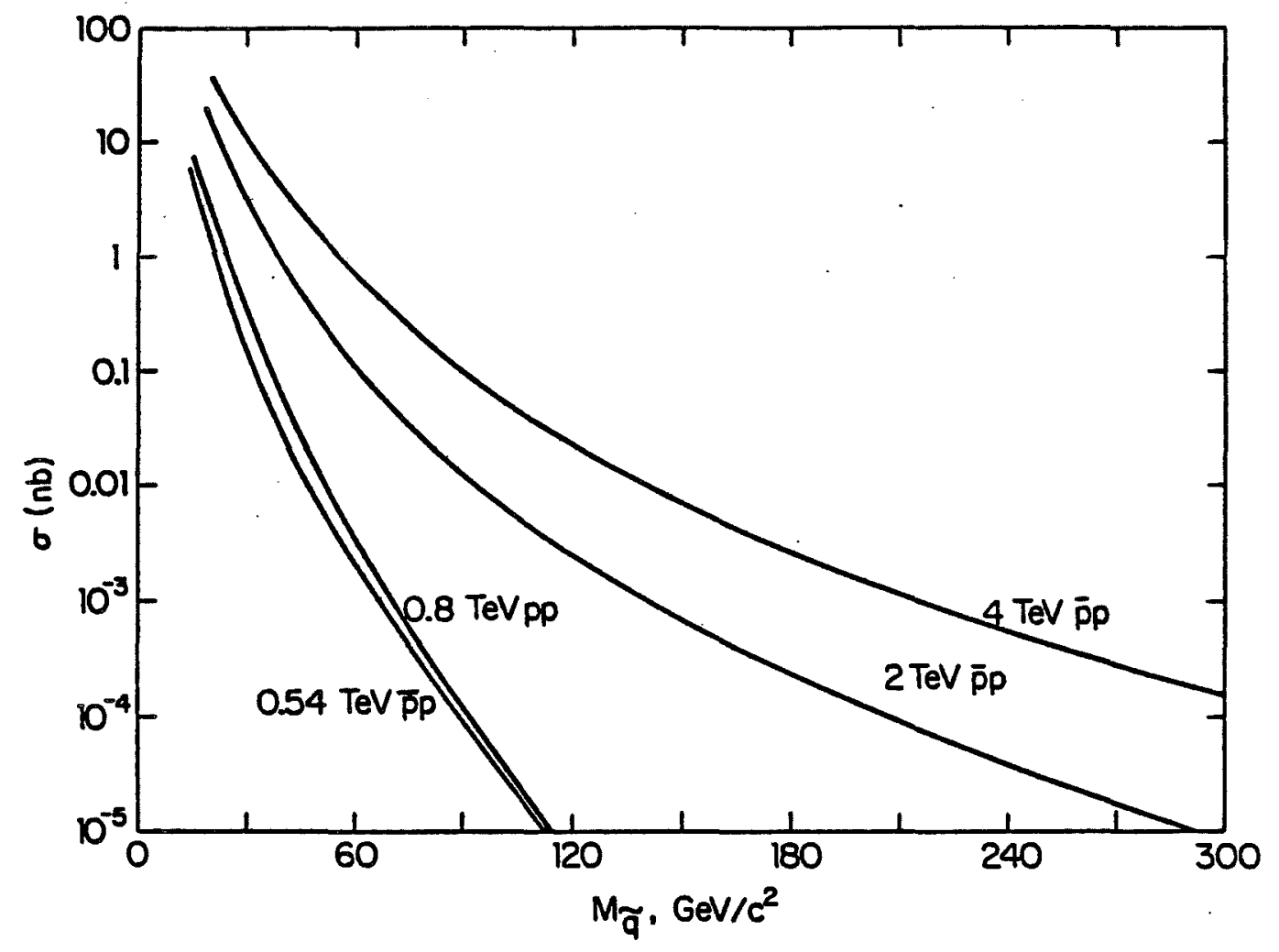

Fig. II-12. Cross sections for pair production of squarks in hadron-hadron collisions. 


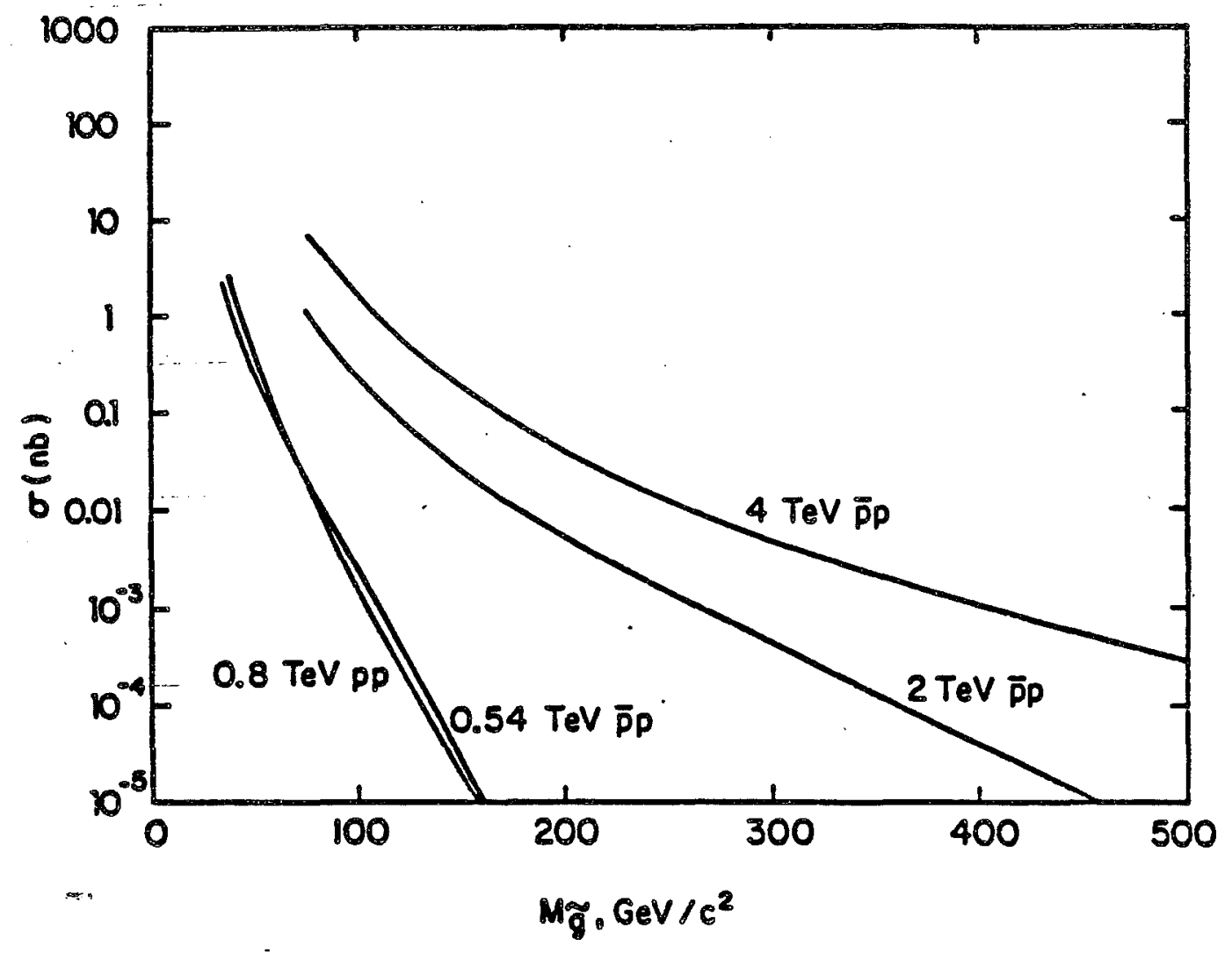

F1g. II-13. Cross sections for pair production of gluinos in hadron-hadron collisions. 
One manifestation of compositeness would be the appearance of form factors for the quarks and leptons. While evidence on this point will be accumulated in both the ep and $\overline{p p}$ modes of the $D C$, another signature may be more teling in the near term. This is the effect of a new contact interaction between quarks and leptons which should emerge if these fermions have constituents.

If quarks and leptons are bound states, ihe eopse that binds the constituents will also mediate new interactions zmong bine bound states. At energies far below the compositeness scale, binese new interactions may be represented as effective contact terms of the form

$$
\delta= \pm \frac{g^{2}}{\Lambda^{2}}(\bar{f} f)\left(\bar{f} \cdot f^{\prime}\right),
$$

where $\Lambda$ is the compositeness scale. It is plausible (since the interactions must be strong) inat $\mathrm{g}^{2} / 4 \pi=1$.

The effect of the contact term on jet production for various values of $\Lambda$ and for both + and - signs in the coupling is shown in Fig. II-14. Under the assumption that detection of a departure from QCD expectations would be noticeable if the deviation is (i) by a factor of two or more, (ii) gives at least 100 events/run variation from expectation, and (iii) gives a detectable non-scaling energy behavior, the sensitivity is indicated in Table II-11. It should be possible to observe a compositeness scale of up to $3 \mathrm{TeV}$ at the DC.

If both the light quarks and muons are composite, then the effects of the contact interaction will modify the usual Drell-Yan cross section. The resurting oross sections are shown in Fig. II-15. The maximum compositeness scaie to which one may expect to be sensitive is shown in Table II-12, under similar assumptions to those made for the hadron jets. The limit set on $\Lambda$ by the DC should be $6 \mathrm{TeV}$.

Finally, there is the possibility in a composite model that excited colored objects may be pair-produced in hadronic collisions. These exotic fermions might be expected to appear with masses of a few hundred $\mathrm{GeV} / \mathrm{c}^{2}$. Table II-13 shows that for color triplets, sextets, and octets the DC provides sensitivity over an interesting range.

\section{E. Parton Luminosities; Summary of pp̄ Opportunities}

High-mass hard collisions are the principal avenue to high-energy parton-parton interactions. Cross sections for hard collisions are characterized by the limiting high-energy behavior

$$
\sigma(\hat{\mathrm{s}})=\mathrm{c} / \hat{\mathrm{s}} \text {, }
$$

where $\hat{s}$ is the squared subenergy for the elementary process and $c$ is a process-dependent number which typically lies between $10^{-3}$ and 1 . The 



F1g. II-14. Deviations from QCD ylelds for jet production induced by quark compositeness._. (a) $4 \mathrm{TeV}$ pp; (b) $2 \mathrm{TeV} \mathrm{pp;} \mathrm{(c)} 0.8$ TeV pp; (d) $0.54 \mathrm{TeV}$ pp。 

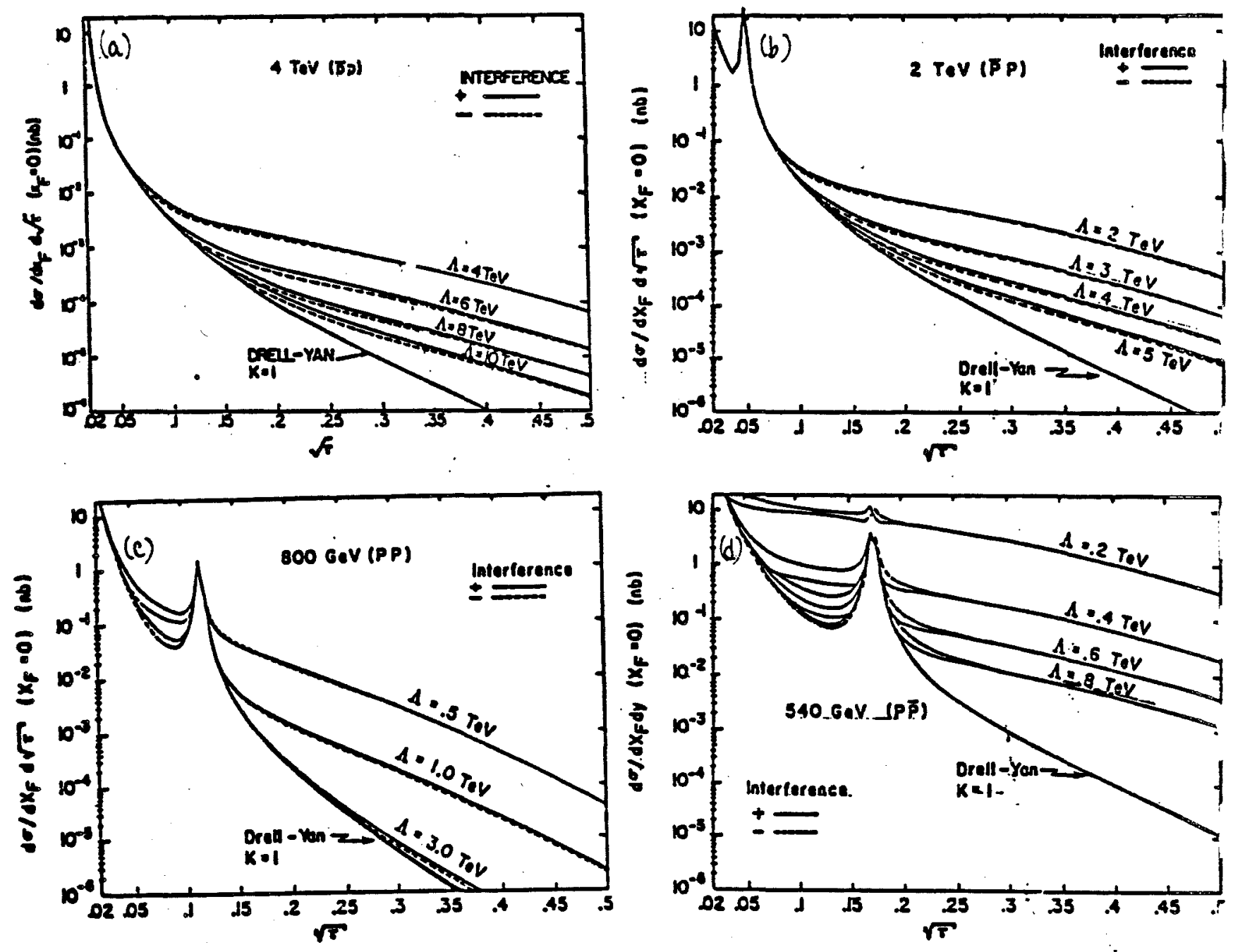

F18. II-15. Deviations from lowest-order Drell-Yan yields induced by quark and lepton compositeness. (a) $4 \mathrm{TeV}$ pp (b) $2 \mathrm{TeV} \overline{\mathrm{p} p}$; (c) $0.8 \mathrm{TeV}$ pp; (d) $0.54 \mathrm{TeV}$ pp. Here $\tau=\mathrm{m}^{2} / \mathrm{s}$. 
number of events $\mathrm{N}$ accumulated in a collider run with integrated luminosity $\int d t \mathscr{L}$ at machine $c . m$. energy $d s$ will be given by

$$
\mathrm{N}=(\mathrm{c} / \hat{\mathrm{s}}) \mathrm{F}(\hat{\mathbf{s}}, \mathbf{s}) \int \mathrm{d} t \mathscr{L},
$$

where $F(\mathbf{S}, \hat{\mathbf{S}})$ is a convolution over parton distribution functions in the colliding beams. For various values of $c$ and for an assumed integrated luminosity of $\int \mathrm{dt} \mathscr{L}=10^{38} \mathrm{~cm}^{-2}$ characteristic of a DC run, we show in Fig. II-16 the value of the maximum subenergy $\sqrt{\mathrm{s}}$ for which $a$ run at mach ine energy $\sqrt{3}$ will accumulate 100 events. We see again in a general way that the natural scale of subenergies to be explored at the DC is typically of order 0.5 to $1.5 \mathrm{TeV}$. This is superior to what can be reached with other colliders.

In summary, the mass scales on which the Dedicated Collider will be sensitive for various processes and new particles, as calculated above, is reviewed in Table II-14. The high physics interest and cost-effectiveness of the DC are apparent.

\section{F. Uncertainties in Rate Estimates}

In spite of the great erforts devoted to the study of deeply inelastic scattering and the extraction of structure functions, important ambiguities remain in the parton distributions. These are especially significant for small values of $x$ and at all $Q^{2}$, and at large values of $x$ for large $Q^{2}$. They arise both from the original parameterizations at modest $Q^{2}$ and from the $Q C D$ evolution to larger $Q^{2}$.

The parton distributions of Owens, Reya, and Duke that we have used for the illustrative calculations in this Section may be characterized as "gluon poor." For most purposes they may be regarded as providing conservative estimates of the cross sections. In the preliminary studies which led to this proposal we have found it useful to consider in addition the Baier, et al. distributions used in the Snowmass study, which represent the opposite extrene of "gluon rich" distributions. [For the Snowmass calculations, $\Lambda=0.1 \mathrm{GeV}$ was used; we take $\Lambda=0.4 \mathrm{GeV}$, the value obtained by Baier, et al. in their fits. This makes little difference in the results.] Although we believe that reality is likely to lie closer to the gluon poor distributions, the more important point is that a comparison of the two distributions provides a measure of the uncertainty of any such calculations in light of current knowledge. It should also be remembered that the calculations we present are all lowest-order estinates subject to their own theoretical uncertainties.

Luminosity coniours for the Baier, et al. distributions are shown in Fig. II-17. The relative importance of $u \bar{u}$ and $g g$ collisions is different from what is displayed in Fig. II-16, but the energy dependence (as reflected in the slopes of the contours) is quite compatible. Thus the the absolute scale probed by a given machine is distribution-dependent, but the relative comparison among machines is rather insensitive to the parion distributions. The last point is 

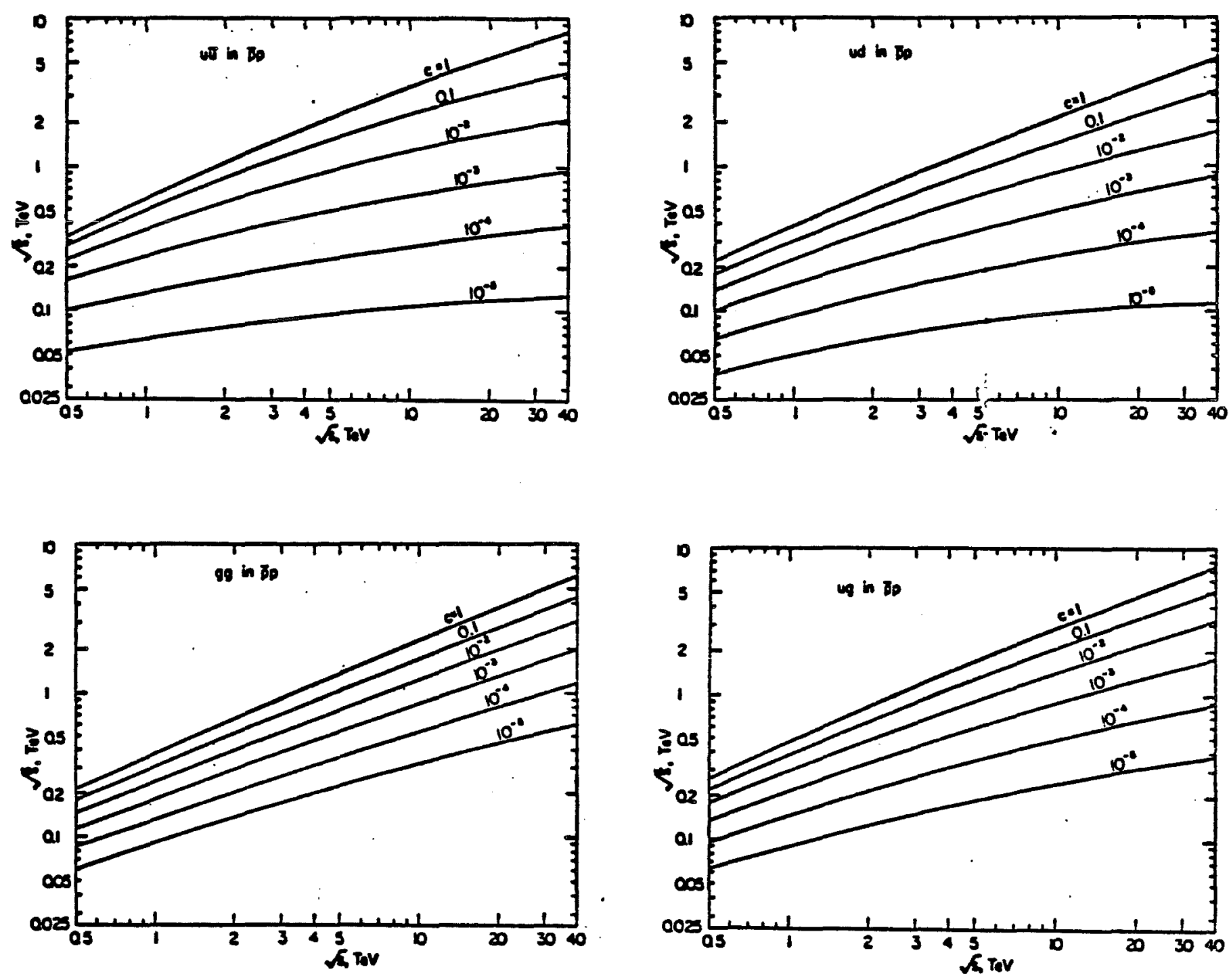

F1g. II-16. Contours in Invariant mass of a hard collision yielding 100 events in running with integrated luminosity $10^{38} \mathrm{~cm}^{-2}$, for hard-scattering cross sections $\sigma(\hat{s})=c / \hat{s}$. 



Fig. II-17 Contours in invariant mass of a hard collision yielding 100 events in running with integrated luminosity $10^{38} \mathrm{~cm}^{-2}$, for hard-scattering cross sections $\sigma(\hat{s})=c / \hat{\mathrm{s}}$ (Baier parton distribution). 
amplified by the Snowmass contour plots (Fig. 4, Snowmass, p. 96), and by Tables II-1A-II-14A, which provide crude assessments of machine capabilities according to the gluon rich distributions. 

Table II-1. Number of standard model intermediate bosons anticipated in hadron-hadron colliders.

\begin{tabular}{lccccc}
\hline Collider & $\sqrt{\mathrm{s}}, \mathrm{TeV}$ & $\int \mathrm{dt} \mathscr{L}^{2} \mathrm{~cm}^{-2}$ & $\mathrm{~W}^{+}$ & $\mathrm{W}^{-}$ & $\mathrm{Z}^{0}$ \\
\hline $\operatorname{DC}(\overline{\mathrm{pp}})$ & 4.0 & $10^{38}$ & $2 \times 10^{6}$ & $2 \times 10^{6}$ & $10^{6}$ \\
$\operatorname{TeV~I}(\overline{\mathrm{pp}})$ & 2.0 & $10^{37}$ & $10^{5}$ & $10^{5}$ & $60 \mathrm{~K}$ \\
& & $10^{38}$ & $10^{6}$ & $10^{6}$ & $600 \mathrm{~K}$ \\
$\mathrm{CBA}(\mathrm{pp})$ & 0.8 & $10^{38}$ & $150 \mathrm{~K}$ & $80 \mathrm{~K}$ & $60 \mathrm{~K}$ \\
& & $10^{40}$ & $15 \times 10^{6}$ & $8 \times 10^{6}$ & $6 \times 10^{6}$ \\
$\operatorname{Spps}$ & 0.54 & $10^{37}$ & $20 \mathrm{~K}$ & $20 \mathrm{~K}$ & $15 \mathrm{~K}$ \\
& & $10^{38}$ & $200 \mathrm{~K}$ & $200 \mathrm{~K}$ & $150 \mathrm{~K}$ \\
\hline
\end{tabular}

Table II-2. Number of gauge boson pairs anticipated in hadron-hadron colliders.

\begin{tabular}{lcccrr}
\hline Collider & $\sqrt{\mathrm{s}}, \mathrm{TeV}$ & $\int \mathrm{dt} \mathcal{L}^{\circ} \mathrm{cm}^{-2}$ & $\mathrm{~W}^{+} \mathrm{W}^{-}$ & $\mathrm{W}^{-} \mathrm{z}^{\circ}$ & $\mathrm{Z}^{\circ} \mathrm{Z}^{\circ}$ \\
\hline $\mathrm{DC}(\overline{\mathrm{pp}})$ & 4.0 & $10^{38}$ & $1.3 \mathrm{~K}$ & 700 & 370 \\
$\operatorname{TeV} \mathrm{I}(\overline{\mathrm{pp}})$ & 2.0 & $10^{37}$ & 60 & 26 & 15 \\
& & $10^{38}$ & 600 & 260 & 150 \\
$\mathrm{CBA}(\mathrm{pp})$ & 0.8 & $10^{38}$ & 8 & 1 & 1 \\
& & $10^{40}$ & 780 & 110 & 140 \\
$\operatorname{Spps}$ & 0.54 & $10^{37}$ & 2 & $<1$ & $<1$ \\
& & $10^{38}$ & 22 & 3 & 3 \\
\hline
\end{tabular}


Table II-1A. Number of standard model intermediate bosons anticipated in hadron-hadron colliders. [Baier distributions]

\begin{tabular}{lccccc}
\hline Collider & $\sqrt{\mathrm{s}}, \mathrm{TeV}$ & $\int \mathrm{d} \mathcal{L}^{\mathrm{V}}, \mathrm{cm}^{-2}$ & $\mathrm{w}^{+}$ & $W^{-}$ & $\mathrm{Z}^{0}$ \\
\hline $\mathrm{DC}(\overline{\mathrm{pp}})$ & 4.0 & $10^{38}$ & $15 \times 10^{6}$ & $15 \times 10^{6}$ & $8 \times 10^{6}$ \\
$\operatorname{TeV~I}(\overline{\mathrm{pp}})$ & 2.0 & $10^{37}$ & $7 \times 10^{5}$ & $7 \times 10^{5}$ & $350 \mathrm{~K}$ \\
& & $10^{38}$ & $7 \times 10^{6}$ & $7 \times 10^{6}$ & $3.5 \times 10^{6}$ \\
$\mathrm{CBA}(\mathrm{pp})$ & 0.8 & $10^{38}$ & $400 \mathrm{~K}$ & $250 \mathrm{~K}$ & $120 \mathrm{~K}$ \\
& & $10^{40}$ & $40 \times 10^{6}$ & $25 \times 10^{6}$ & $12 \times 10^{6}$ \\
$\operatorname{Spps}$ & 0.54 & $10^{37}$ & $60 \mathrm{~K}$ & $60 \mathrm{~K}$ & $20 \mathrm{~K}$ \\
& & $10^{38}$ & $600 \mathrm{~K}$ & $600 \mathrm{~K}$ & $200 \mathrm{~K}$ \\
\hline
\end{tabular}

Table II-2A. Number of gauge boson pairs anticipated in hadron-hadron colliders. [Baier distributions]

\begin{tabular}{lccccc}
\hline Collider & $\sqrt{\mathrm{s}}, \mathrm{TeV}$ & $\int \mathrm{dtd}, \mathrm{cm}^{-2}$ & $\mathrm{~W}^{+} \mathrm{W}^{-}$ & $\mathrm{W}^{-} \mathrm{Z}^{0}$ & $\mathrm{Z}^{\circ} \mathrm{Z}^{0}$ \\
\hline $\mathrm{DC}(\overline{\mathrm{pp}})$ & 4.0 & $10^{38}$ & $10 \mathrm{~K}$ & $5.1 \mathrm{~K}$ & $2.9 \mathrm{~K}$ \\
$\mathrm{TeV} \mathrm{I}(\overline{\mathrm{pp}})$ & 2.0 & $10^{37}$ & 240 & 96 & 61 \\
& & $10^{38}$ & $2.4 \mathrm{~K}$ & 960 & 608 \\
$\mathrm{CBA}(\mathrm{pp})$ & 0.8 & $10^{38}$ & 9 & 1.5 & 1.4 \\
& & $10^{40}$ & 920 & 150 & 140 \\
$\operatorname{Spps}$ & 0.54 & $10^{37}$ & 2 & .3 & .3 \\
& & $10^{38}$ & 17 & 3 & 3 \\
\hline
\end{tabular}


Table II-3. Maximum mass (in $\mathrm{GeV} / \mathrm{c}^{2}$ ) for production of 100 Higgs boson events in hadron-hadron colliders.

\begin{tabular}{lccccc}
\hline & & \multicolumn{4}{c}{$\mathrm{M}_{\mathrm{H}}(\mathrm{max})$} \\
Collider & $\sqrt{\mathrm{s}}, \mathrm{TeV}$ & $\int \mathrm{d}+\mathcal{L}, \mathrm{cm}^{-2}$ & $\mathrm{~m}_{t}=20$ & $\mathrm{~m}_{t}=40$ & $\mathrm{~m}_{t}=80$ \\
\hline $\operatorname{DC}(\overline{\mathrm{pp}})$ & 4.0 & $10^{38}$ & 135 & 175 & 220 \\
$\operatorname{TeV~I}(\overline{\mathrm{pp}})$ & 2.0 & $10^{37}$ & 50 & 35 & 35 \\
& & $10^{38}$ & 100 & 115 & 80 \\
$\mathrm{CBA}(\mathrm{pp})$ & 0.8 & $10^{38}$ & 55 & 50 & 45 \\
& & $10^{40}$ & 125 & 150 & 140 \\
$\operatorname{Spps}$ & 0.54 & $10^{37}$ & 25 & 20 & 20 \\
& & $10^{38}$ & 40 & 35 & 40 \\
\hline
\end{tabular}

Table II-4. Maximum quark mass (in $\mathrm{GeV} / \mathrm{c}^{2}$ ) for production of 100 quark-antiquark pairs in hadron-hadron colliders.

\begin{tabular}{lccc}
\hline Collider & $\sqrt{\mathrm{s}}, \mathrm{TeV}$ & $\int \mathrm{dt} \mathcal{L}, \mathrm{cm}^{-2}$ & $\mathrm{M}_{\mathrm{q}}$ (max) \\
\hline $\operatorname{DC}(\overline{\mathrm{pp}})$ & 4.0 & $10^{38}$ & 320 \\
$\operatorname{TeV~I}(\overline{\mathrm{pp}})$ & 2.0 & $10^{37}$ & 140 \\
& & $10^{38}$ & 220 \\
$\mathrm{CBA}(\mathrm{pp})$ & 0.8 & $10^{38}$ & 85 \\
& & $10^{40}$ & 140 \\
$\operatorname{Spps}$ & 0.54 & $10^{37}$ & 70 \\
& & $10^{38}$ & 95 \\
\hline
\end{tabular}


Table II-3A. Maximum mass (in GeV/ $/ \mathrm{c}^{2}$ ) for production of 100 Higgs boson events in hadron-hadron colliders. [Baier distributions]

\begin{tabular}{lcccc}
\hline Col11der & $\sqrt{\mathrm{s}}, \mathrm{TeV}$ & $\int \mathrm{d} \mathcal{L}^{2}, \mathrm{~cm}^{-2}$ & $\mathrm{~m}_{\mathrm{t}}=20$ & $\mathrm{~m}_{\mathrm{t}}=80$ \\
\hline $\operatorname{DC}(\overline{\mathrm{pp}})$ & 4.0 & $10^{38}$ & 170 & 340 \\
$\operatorname{TeV~I}(\overline{\mathrm{pp}})$ & 2.0 & $10^{37}$ & 65 & 40 \\
& & $10^{38}$ & 120 & 215 \\
$\operatorname{CBA}(\mathrm{pp})$ & 0.8 & $10^{38}$ & 70 & 45 \\
& & $10^{40}$ & 170 & 260 \\
$\operatorname{Spps}$ & 0.54 & $10^{37}$ & 20 & 20 \\
& & $10^{38}$ & 50 & 35 \\
\hline
\end{tabular}

Table II-4A. Maximum quark mass (in GeV/c $\mathrm{c}^{2}$ ) for production of 100 quark-antiquark pairs in hadron-hadron colliders. [Baier]

\begin{tabular}{|c|c|c|c|}
\hline Collider & $\sqrt{\mathrm{s}}, \mathrm{TeV}$ & $\int \mathrm{d} b \mathcal{X}, \mathrm{cm}^{-2}$ & $M_{q}(\max )$ \\
\hline$D C(\overline{p p})$ & 4.0 & $10^{38}$ & 395 \\
\hline $\mathrm{TeV} I(\overline{p p})$ & 2.0 & $\begin{array}{l}10^{37} \\
10^{38}\end{array}$ & $\begin{array}{l}170 \\
240\end{array}$ \\
\hline $\mathrm{CBA}(p p)$ & 0.8 & $\begin{array}{l}10^{38} \\
10^{40}\end{array}$ & $\begin{array}{l}110 \\
205\end{array}$ \\
\hline$s \overline{p p} s$ & 0.54 & $\begin{array}{l}10^{37} \\
10^{38}\end{array}$ & $\begin{array}{l}70 \\
95 \\
\end{array}$ \\
\hline
\end{tabular}


Table II-5. Maximum mass (in GeV/ $\mathrm{c}^{2}$ ) for production of $100 \mathrm{~W}^{ \pm}$or $\mathrm{Z}^{\circ}$ gauge bosons in hadron-hadron colliders.

\begin{tabular}{lccc}
\hline Collider & $\sqrt{\mathrm{s}}, \mathrm{TeV}$ & $\int \mathrm{dt} \mathcal{L}, \mathrm{cm}^{-2}$ & $\mathrm{M}^{(\max )}$ \\
\hline $\operatorname{DC}(\overline{\mathrm{pp}})$ & 4.0 & $10^{38}$ & 1200 \\
$\operatorname{TeV} I(\overline{\mathrm{pp}})$ & 2.0 & $10^{37}$ & 500 \\
& & $10^{38}$ & 750 \\
$\mathrm{CBA}(\mathrm{pp})$ & 0.8 & $10^{38}$ & 300 \\
& & $10^{40}$ & 400 \\
$\operatorname{Spps}$ & 0.54 & $10^{37}$ & 200 \\
& & $10^{38}$ & 275 \\
\hline
\end{tabular}

Table II-6. Maximum transverse momentum (in GeV/c) for production of 100 hadron jets per unit rapidity at $\mathrm{y}_{\mathrm{m}}=0$, per $\mathrm{GeV} / \mathrm{c}$ transverse momentum, in hadron-hadron coiliders.

\begin{tabular}{lccc}
\hline Collider & $\sqrt{\mathrm{s}}, \mathrm{TeV}$ & $\int \mathrm{dt} \mathcal{X}, \mathrm{cm}^{-2}$ & $\mathrm{P}_{\perp}{ }^{(\max )}$ \\
\hline $\operatorname{DC}(\overline{\mathrm{pp}})$ & 4.0 & $10^{38}$ & 350 \\
$\operatorname{TeV} I(\overline{\mathrm{pp}})$ & 2.0 & $10^{37}$ & 175 \\
& & $10^{38}$ & 250 \\
$\mathrm{CBA}(\mathrm{pp})$ & 0.8 & $10^{38}$ & 140 \\
& & $10^{40}$ & 210 \\
$\operatorname{SppS}$ & 0.54 & $10^{37}$ & 95 \\
& & $10^{38}$ & 115 \\
\hline
\end{tabular}
Table II-7. Maximum mass (in $\mathrm{GeV} / \mathrm{c}^{2}$ ) for single production of $100 \mathrm{n}_{\mathrm{T}}$
events in hadron-hadron collisions.

\begin{tabular}{lcll}
\hline Collider & $\sqrt{\mathrm{s}}, \mathrm{TeV}$ & $\int \mathrm{dt} \mathscr{L}, \mathrm{cm}^{-2}$ & $M^{(\max )}$ \\
\hline $\operatorname{DC}(\overline{\mathrm{pp}})$ & 4.0 & $10^{38}$ & 640 \\
$\operatorname{TeV~I}(\overline{\mathrm{pp}})$ & 2.0 & $10^{37}$ & 210 \\
& & $10^{38}$ & 340 \\
$\mathrm{CBA}(\mathrm{pp})$ & 0.8 & $10^{38}$ & 160 \\
& & $10^{40}$ & 300 \\
\hline
\end{tabular}


Table II-5A. Maximum mass (in $\mathrm{GeV} / \mathrm{c}^{2}$ ) for production of $100 \mathrm{w}^{ \pm}$or $\mathrm{Z}^{0}$ gauge bosons in hadron-hadron colliders. [Baier]

\begin{tabular}{lccc}
\hline Collider & $\sqrt{s}, \operatorname{TeV}$ & $\int \mathrm{dt} \mathcal{L}, \mathrm{cm}^{-2}$ & $M^{(\mathrm{max})}$ \\
\hline $\operatorname{DC}(\overline{\mathrm{pp}})$ & 4.0 & $10^{38}$ & 1180 \\
$\operatorname{TeV} I(\overline{\mathrm{pp}})$ & 2.0 & $10^{39}$ & 530 \\
& & $10^{38}$ & 730 \\
$\mathrm{CBA}(\mathrm{pp})$ & 0.8 & $10^{38}$ & 260 \\
& & $10^{40}$ & 360 \\
$\operatorname{Spps}$ & 0.54 & $10^{37}$ & 210 \\
& & $10^{38}$ & 270 \\
\hline
\end{tabular}

Table II-6A. Maximum transverse momentum (in GeV/c) for producition of 100 hadron jets per unit rapidity at $\mathrm{y}_{\mathrm{c}}=0$, per $\mathrm{GeV} / \mathrm{c}$ transverse momentum, in hadron-hadron collinders. [Baier]

\begin{tabular}{lccl}
\hline Collider & $\sqrt{\mathrm{s}}$, eV & $\int \mathrm{dt} \mathcal{L} \mathrm{cm}^{-2}$ & $P_{\mathrm{T}}{ }^{(\max )}$ \\
\hline $\operatorname{DC}(\overline{\mathrm{pp}})$ & 4.0 & $10^{38}$ & 500 \\
$\operatorname{TeV} I(\overline{\mathrm{pp}})$ & 2.0 & $10^{37}$ & 225 \\
& & $10^{38}$ & 300 \\
$\mathrm{CBA}(\mathrm{pp})$ & 0.8 & $10^{38}$ & 160 \\
& & $10^{40}$ & 235 \\
$\operatorname{Spps}$ & 0.54 & $10^{37}$ & 100 \\
& & $10^{38}$ & 120 \\
\hline
\end{tabular}
Table II-7A. Maximum mass (in GeV/c $\mathrm{c}^{2}$ ) for single production of $100 \mathrm{n}_{\mathrm{T}}$
events in hadron-hadron collisions. [Baier]

\begin{tabular}{lcll}
\hline Collider & $\sqrt{3}$, TeV & $\int d t \mathscr{C}, \mathrm{cos}^{-2}$ & $M^{(\max )}$ \\
\hline $\operatorname{DC}(\overline{\mathrm{p} p})$ & 4.0 & $10^{38}$ & $1.4 \mathrm{TeV} / \mathrm{c}^{2}$ \\
$\operatorname{TeV} I(\overline{\mathrm{pp}})$ & 2.0 & $10^{37}$ & 440 \\
& & $10^{38}$ & 760 \\
$\mathrm{CBA}(\mathrm{pp})$ & 0.8 & $10^{38}$ & 280 \\
& & $10^{40}$ & 490 \\
\hline
\end{tabular}


Table II-8. Maximum mass (in $\mathrm{GeV} / \mathrm{c}^{2}$ ) for production of 100 technihadron pairs in hadron-hadron collisions.

\begin{tabular}{lccccc}
\hline & \multicolumn{5}{c}{$\mathrm{M}_{\mathrm{T}}^{(\mathrm{max})}$} \\
Collider & $\sqrt{\mathrm{s}}, \mathrm{TeV}$ & $\int \mathrm{dt} \mathcal{L}, \mathrm{cm}^{-2}$ & $\mathrm{P}_{3}($ leptoquark $)$ & $\mathrm{P}_{6}$ & $\mathrm{P}_{8}\left(n_{\mathrm{T}}\right)$ \\
\hline $\operatorname{DC}(\overline{\mathrm{pp}})$ & 4.0 & $10^{38}$ & 260 & 330 & 345 \\
$\operatorname{TeV~I}(\overline{\mathrm{pp}})$ & 2.0 & $10^{37}$ & 100 & 170 & 175 \\
& & $10^{38}$ & 170 & 240 & 240 \\
$\mathrm{CBA}(\mathrm{pp})$ & 0.8 & $10^{38}$ & 80 & 100 & 100 \\
& & $10^{40}$ & 120 & 150 & 150 \\
\hline
\end{tabular}

Table II-9. Maximum squark mass (in $G e V / \mathrm{c}^{2}$ ) for production of 100 squark-antisquark pairs in hadron-hadron colliders.

\begin{tabular}{lccc}
\hline Collider & $\sqrt{\mathrm{s}}, \mathrm{TeV}$ & $\int \mathrm{dt} \mathcal{L}^{0} \mathrm{~cm}^{-2}$ & $M_{\tilde{\mathrm{q}}}^{(\max )}$ \\
\hline $\operatorname{DC}(\overline{\mathrm{pp}})$ & 4.0 & $10^{38}$ & 215 \\
$\operatorname{TeV~I}(\overline{\mathrm{pp}})$ & 2.0 & $10^{37}$ & 100 \\
& & $10^{38}$ & 155 \\
$\mathrm{CBA}(\mathrm{pp})$ & 0.8 & $10^{38}$ & 70 \\
& & $10^{40}$ & 120 \\
$\operatorname{Spps}$ & 0.54 & $10^{37}$ & 45 \\
& & $10^{38}$ & 65 \\
\hline
\end{tabular}

Table II-10. Maximum gluino mass (in $\mathrm{GeV} / \mathrm{c}^{2}$ ) for production of 100 gluino pairs in hadron-hadron colliders.

\begin{tabular}{lccc}
\hline Collider & $\sqrt{\mathrm{s}}, \mathrm{TeV}$ & $\int \mathrm{dt} \mathscr{L}, \mathrm{cm}^{-2}$ & $\mathrm{M}_{\tilde{\mathrm{g}}}{ }^{(\max )}$ \\
\hline $\operatorname{DC}(\overline{\mathrm{p} p})$ & 4.0 & $10^{38}$ & 400 \\
$\operatorname{TeV~I}(\overline{\mathrm{pp}})$ & 2.0 & $10^{37}$ & 175 \\
& & $10^{38}$ & 275 \\
$\mathrm{CBA}(\mathrm{pp})$ & 0.8 & $10^{38}$ & 110 \\
& & $10^{40}$ & 165 \\
$\operatorname{Spps}$ & 0.54 & $10^{37}$ & 80 \\
& & $10^{38}$ & 110 \\
\hline
\end{tabular}


Table II-8A. Maximum mass (in $G e V / c^{2}$ ) for production of 100 technihadron pairs in hadron-hadron collisions. [Baier distributions ]

\begin{tabular}{|c|c|c|c|c|c|}
\hline Collider & $\sqrt{\mathrm{s}}, \mathrm{TeV}$ & $\int \mathrm{d} t \mathcal{L}, \mathrm{cm}^{-2}$ & $\begin{array}{c}M_{T}^{(\max )} \\
\mathrm{P}_{3} \text { (leptoquark) } \\
\end{array}$ & $\mathrm{P}_{6}$ & $P_{8}\left(n_{T}\right)$ \\
\hline $\mathrm{DC}(\overline{\mathrm{p} p})$ & 4.0 & $10^{38}$ & 340 & 500 & 500 \\
\hline$T \in V I(\overline{p p})$ & 2.0 & $\begin{array}{l}10^{37} \\
10^{38}\end{array}$ & $\begin{array}{l}140 \\
210\end{array}$ & $\begin{array}{l}230 \\
320\end{array}$ & $\begin{array}{l}230 \\
320\end{array}$ \\
\hline $\mathrm{CBA}(\mathrm{pp})$ & 0.8 & $\begin{array}{l}10^{38} \\
10^{40}\end{array}$ & $\begin{array}{l}100 \\
170\end{array}$ & $\begin{array}{l}150 \\
210\end{array}$ & $\begin{array}{l}150 \\
210\end{array}$ \\
\hline
\end{tabular}

Table II-9A. Maximum squark mass (in GeV/o ${ }^{2}$ ) for production of 100 squark-antisquark pairs in hadron-hadron colliders . [Baier]

\begin{tabular}{lccc}
\hline Collider & $\sqrt{\mathrm{s}}, \mathrm{TeV}$ & $\int \mathrm{d} \mathcal{X}_{,}, \mathrm{cm}^{-2}$ & $M_{\tilde{\mathrm{q}}}^{(\max )}$ \\
\hline $\operatorname{DC}(\overline{\mathrm{pp}})$ & 4.0 & $10^{38}$ & 305 \\
$\operatorname{TeV} I(\overline{\mathrm{pp}})$ & 2.0 & $10^{37}$ & 120 \\
& & $10^{38}$ & 190 \\
$\operatorname{CBA}(\mathrm{pp})$ & 0.8 & $10^{38}$ & 100 \\
& & $10^{40}$ & 170 \\
\hline
\end{tabular}

Table II-10A. Maximum gluino mass (in $\mathrm{GeV} / \mathrm{c}^{2}$ ) for production of 100 gluino pairs in hadron-hadron colliders. [Baier]

\begin{tabular}{lccc}
\hline Collider & $\sqrt{\mathrm{s}}, \mathrm{TeV}$ & $\int \mathrm{dtZ}, \mathrm{cm}^{-2}$ & $\mathrm{M}_{\tilde{\mathrm{g}}}(\max )$ \\
\hline DC $(\overline{\mathrm{pp}})$ & 4.0 & $10^{38}$ & 500 \\
TeV I $(\overline{\mathrm{pp}})$ & 2.0 & $10^{37}$ & 240 \\
& & $10^{38}$ & 315 \\
$\mathrm{CBA}(\mathrm{pp})$ & 0.8 & $10^{38}$ & 155 \\
& & $10^{40}$ & 215 \\
\hline
\end{tabular}


Table II-11. Limits on a compositeness scale in the production of hadron jets.

\begin{tabular}{lccc}
\hline Collider & $\sqrt{\mathrm{s}}, \mathrm{TeV}$ & $\int \mathrm{dt} \mathcal{L}, \mathrm{cm}^{-2}$ & $\Lambda^{\mathrm{LH}}, \mathrm{TeV}$ \\
\hline $\mathrm{DC}(\overline{\mathrm{pp}})$ & 4.0 & $10^{38}$ & 3.0 \\
$\operatorname{TeV} I(\overline{\mathrm{pp}})$ & 2.0 & $10^{37}$ & 1.2 \\
& & $10^{38}$ & 1.5 \\
$\mathrm{CBA}(\mathrm{pp})$ & 0.8 & $10^{38}$ & 1.2 \\
& & $10^{40}$ & 1.8 \\
$\operatorname{Spps}$ & 0.54 & $10^{37}$ & 0.4 \\
& & $10^{38}$ & 0.6 \\
\hline
\end{tabular}

Table II-12. Limits on a compositeness scale in Drell-Yan production of massive lepton pairs.

\begin{tabular}{lccc}
\hline Collider & $\sqrt{\mathrm{s}}, \mathrm{TeV}$ & $\int \mathrm{dt} \mathcal{Z}, \mathrm{cm}^{-2}$ & $\Lambda^{\mathrm{LH}}, \mathrm{TeV}$ \\
\hline $\operatorname{DC}(\overline{\mathrm{pp}})$ & 4.0 & $10^{38}$ & 6.0 \\
$\operatorname{TeV} I(\overline{\mathrm{pp}})$ & 2.0 & $10^{37}$ & 2.5 \\
& & $10^{38}$ & 4.0 \\
$\operatorname{CBA}(\mathrm{pp})$ & 0.8 & $10^{38}$ & 1.0 \\
& & $10^{40}$ & 2.0 \\
$\operatorname{Spps}$ & 0.54 & $10^{37}$ & 0.8 \\
& & $10^{38}$ & 1.2 \\
\hline
\end{tabular}

Table II-13. Maximum mass (in $\mathrm{GeV} / \mathrm{c}^{2}$ ) for production of 100 pairs of excited colored fermions in hadron-hadron colliders.

\begin{tabular}{lccccc}
\hline & & \multicolumn{3}{c}{$M^{(\max )}$} \\
Collider & $\sqrt{\mathrm{s}}, \mathrm{TeV}$ & $\int \mathrm{dt} \mathrm{I}^{2} \mathrm{~cm}^{-2}$ & $\mathrm{q}_{3}^{*}$ & $\mathrm{q}_{6}^{*}$ & $\mathrm{q}_{8}^{*}$ \\
\hline $\operatorname{DC}(\overline{\mathrm{pp}})$ & 4.0 & $10^{38}$ & 325 & 440 & 455 \\
$\operatorname{TeV~I}(\overline{\mathrm{pp}})$ & 2.0 & $10^{37}$ & 140 & 200 & 205 \\
& & $10^{38}$ & 220 & 285 & 290 \\
$\operatorname{CBA}(\mathrm{pp})$ & 0.8 & $10^{38}$ & 85 & 105 & 110 \\
\hline
\end{tabular}


Table II-11A. Limits on a compositeness scale in the production of hadron jets. [Baier distributions]

\begin{tabular}{lccc}
\hline Collider & $\sqrt{\mathrm{s}, \mathrm{TeV}}$ & $\int \mathrm{dt}^{\mathcal{Z}, \mathrm{cm}^{-2}}$ & $\Lambda^{\mathrm{LH}}, \mathrm{TeV}$ \\
\hline $\operatorname{DC}(\overline{\mathrm{pp}})$ & 4.0 & $10^{38}$ & 2.2 \\
$\operatorname{TeV} \mathrm{I}(\overline{\mathrm{pp}})$ & 2.0 & $10^{37}$ & 1.2 \\
& & $10^{38}$ & 1.6 \\
$\operatorname{CBA}(\mathrm{pp})$ & 0.8 & $10^{38}$ & 1.2 \\
& & $10^{40}$ & 1.7 \\
$\operatorname{SppS}$ & 0.54 & $10^{37}$ & 0.4 \\
\hline
\end{tabular}

Table II-12A. Limits on a compositeness scale in Drell-Yan production of massive lepton pairs. [Baier distributions]

\begin{tabular}{lccc}
\hline Collider & $\sqrt{\mathrm{s}, \mathrm{TeV}}$ & $\int \mathrm{d} \mathcal{L}^{2} \mathrm{~cm}^{-2}$ & $\Lambda^{\mathrm{LH}}, \mathrm{TeV}$ \\
\hline $\mathrm{DC}(\overline{\mathrm{pp}})$ & 4.0 & $10^{38}$ & 5.5 \\
$\operatorname{TeV~I}(\overline{\mathrm{pp}})$ & 2.0 & $10^{37}$ & 2.0 \\
& & $10^{38}$ & 3.0 \\
$\mathrm{CBA}(\mathrm{pp})$ & 0.8 & $10^{38}$ & 1.2 \\
& & $10^{40}$ & 2.0 \\
$\operatorname{Spps}$ & 0.54 & $10^{37}$ & 0.8 \\
& & $10^{38}$ & 1.2 \\
\hline
\end{tabular}

Table II-13A. Maximum mass (in $\mathrm{GeV} / \mathrm{c}^{2}$ ) for production of 100 pairs of excited colored fermions in hadron-hadron colliders. [Baier]

\begin{tabular}{lccccc}
\hline & & \multicolumn{3}{c}{$\mathrm{M}^{\text {(max) }}$} \\
Collider & $\sqrt{\mathrm{s}}, \mathrm{TeV}$ & $\int \mathrm{d} t \mathcal{L}^{2} \mathrm{~cm}^{-2}$ & $\mathrm{q}_{3}^{*}$ & $\mathrm{q}_{6}^{*}$ & $\mathrm{q}_{8}^{*}$ \\
\hline $\operatorname{DC}(\overline{\mathrm{pp}})$ & 4.0 & $10^{\mathbf{3 8}}$ & 395 & 575 & 580 \\
$\operatorname{TeV~I}(\overline{\mathrm{pp}})$ & 2.0 & $10^{37}$ & 170 & 255 & 260 \\
& & $10^{38}$ & 240 & 345 & 350 \\
$\operatorname{CBA}(\mathrm{pp})$ & 0.8 & $10^{38}$ & 110 & 160 & 160 \\
& & $10^{40}$ & 205 & 215 & 220 \\
\hline
\end{tabular}


Table II-14. Mass limits attainable in the DC for production of 100 events at $\int \mathrm{dt} L=10^{38} \mathrm{~cm}^{-2}$.

\begin{tabular}{|c|c|c|c|}
\hline Particle & DC & $\begin{array}{r}\text { Mass Iimit, } \\
\operatorname{TeV} I\left(10^{37}\right)\end{array}$ & $\begin{array}{l}\mathrm{V} / \mathrm{c}^{2} \\
\mathrm{CBA}\left(10^{40}\right)\end{array}$ \\
\hline \multicolumn{4}{|l|}{ Standard model: } \\
\hline Higgs scalar & $135-220$ & $35-50$ & $125-150$ \\
\hline Heavy fermion & 320 & 140 & 140 \\
\hline Jet pair mass & $>700$ & 350 & 420 \\
\hline \multicolumn{4}{|l|}{ New gauge bosons: } \\
\hline$W^{\prime}$ or $\mathrm{Z}^{\prime}$ & 1200 & 500 & 400 \\
\hline \multicolumn{4}{|c|}{ Supersymmetric partners: } \\
\hline squark & 215 & 100 & 120 \\
\hline gluino & 400 & 175 & 165 \\
\hline \multicolumn{4}{|l|}{ Techniparticles: } \\
\hline octet & 345 & 175 & 150 \\
\hline sextet & 330 & 170 & 150 \\
\hline triplet & 260 & 100 & 120 \\
\hline \multicolumn{4}{|l|}{ Higgs-like scalars } \\
\hline $\mathrm{P}$ & 640 & 210 & 300 \\
\hline $\mathrm{P}_{0}^{8}$ & 400 & 110 & 230 \\
\hline \multicolumn{4}{|c|}{ Compositeness (hadron jets) } \\
\hline $\begin{array}{l}\text { LH scale } \\
\text { RH scale }\end{array}$ & $\begin{array}{l}3000 \\
2500\end{array}$ & 1200 & 1800 \\
\hline
\end{tabular}


Table II-14A. Mass limits attainable in the DC for production of 100 events at $\int \mathrm{dt} \mathscr{L}=10^{38} \mathrm{~cm}^{-2}$. [Baier distribitions]

\begin{tabular}{|c|c|c|c|}
\hline Particle & DC & $\begin{array}{l}\text { Mass Iimit, } \\
\operatorname{TeV} \operatorname{I}\left(10^{37}\right)\end{array}$ & )$^{\mathrm{GeV} / \mathrm{c}^{2}}$ \\
\hline $\begin{array}{l}\text { Standard model: } \\
\text { Higgs scalar } \\
\text { Heavy fermion } \\
\text { Jet pair mass }\end{array}$ & $\begin{array}{l}170-340 \\
395 \\
1000\end{array}$ & $\begin{array}{l}40-65 \\
170 \\
450\end{array}$ & $\begin{array}{l}170-260 \\
205 \\
470\end{array}$ \\
\hline $\begin{array}{c}\text { New gauge bosons: } \\
W^{\prime} \text { or } Z^{\prime}\end{array}$ & 1200 & 530 & 360 \\
\hline $\begin{array}{l}\text { Supersymmetric partners: } \\
\text { squark } \\
\text { gluino }\end{array}$ & $\begin{array}{l}300 \\
500\end{array}$ & $\begin{array}{l}120 \\
240\end{array}$ & $\begin{array}{l}170 \\
215\end{array}$ \\
\hline $\begin{array}{l}\text { Techniparticles: } \\
\text { octet } \\
\text { sextet } \\
\text { triplet }\end{array}$ & $\begin{array}{l}500 \\
500 \\
340\end{array}$ & $\begin{array}{l}230 \\
230 \\
140\end{array}$ & $\begin{array}{l}210 \\
210 \\
170\end{array}$ \\
\hline $\begin{array}{l}\text { Higgs-like scalars } \\
\mathrm{P} \\
\mathrm{P}_{0}^{8} \\
\end{array}$ & $\begin{array}{r}1400 \\
960\end{array}$ & $\begin{array}{l}440 \\
190\end{array}$ & $\begin{array}{l}490 \\
300\end{array}$ \\
\hline $\begin{array}{l}\text { Compositeness (hadron jets) } \\
\text { LH scale } \\
\text { RH scale }\end{array}$ & $\begin{array}{l}2200 \\
1800\end{array}$ & 1200 & 1700 \\
\hline
\end{tabular}




\section{G. Electron-Proton Physics}

In Phase I of an ep physics facility at the Dedicated Collider ( 10 GeV electrons (positrons) on $2 \mathrm{TeV}$ protons (antiprotons)) we enter a new kinematical region where electromagnetic and weak interactions become of comparable strength. The effects of the $t$ channel exchange of $W^{\prime} s$ and Z's are seen clearly, and detailed studies of both $S U(2) \times U(1)$ and $Q C D$ are possible. An ep facility provides complementary physics to both the $e^{t} e^{-}$physics of LEP and SLC and the p p physics we have been discussing. In addition, we are able to search for possible modifications to the standard model--detection of right-handed currents, neutral heavy leptons, leptoquarks, quark substructure, and composite model interactions.

With the envisioned Phase II ( $40 \mathrm{GeV}$ electron ring) we could concentrate on extending the search for new phenomena and heavy particles well beyond those accessible even at LEP II.

Below we will discuss the physics opportunities at an ep facility operating at $s=8 \times 10^{4} \mathrm{GeV}^{2}$ with an integrated luminosity of $5 \times 10^{38} \mathrm{~cm}^{-2}$ in a standard 1 yr run.

\section{Standard Physics: QCD}

An Initial program at the ep facility will of course study the total cross sections and structure functions. Knowledge of the structure functions at small $x$ and large $Q^{2}$ will provide valuable information for use in the study of $p \bar{p}$ physics. The cross section for the one photon contribution which dominated the neutral process can be written

$$
d \sigma=\frac{4 \pi \alpha^{2}}{Q^{2}}\left[1-Q^{2} / s x+1 / 2\left(Q^{2} / s x\right)^{2}\right] F_{2}\left(x, Q^{2}\right) \frac{d x}{x} \frac{d Q^{2}}{Q^{2}}
$$

Since all $s$ dependence comes from the bracketed factor which only varies by a factor of 2 over the whole kinematic range $\left(0<Q^{2}<s x\right)$, we conclude that although increasing $s$ increases the accessible $Q^{2}$ at a given $x$, it does not appreciably increase the yield of events in a given accessible $\mathrm{X}$ and $\mathrm{Q}^{2}$ bin.

The range of $Q^{2}-x$ which is accessible with the ep collider is shown in Fig. II-18.

Another aspect of standard QCD measurements which will complement the $p \bar{p}$ mode is the ability in an ep machine to correlate the energy and momentum of the struck quark with the properties of the resulting jet. The kinematic eonstraints obtained from observing the outgoing lepton in neutral current reactions allows a study of the dressing of the quark into the jet of hadrons and a fuller understanding of the mechanism of jet broadening for energetic quarks. 


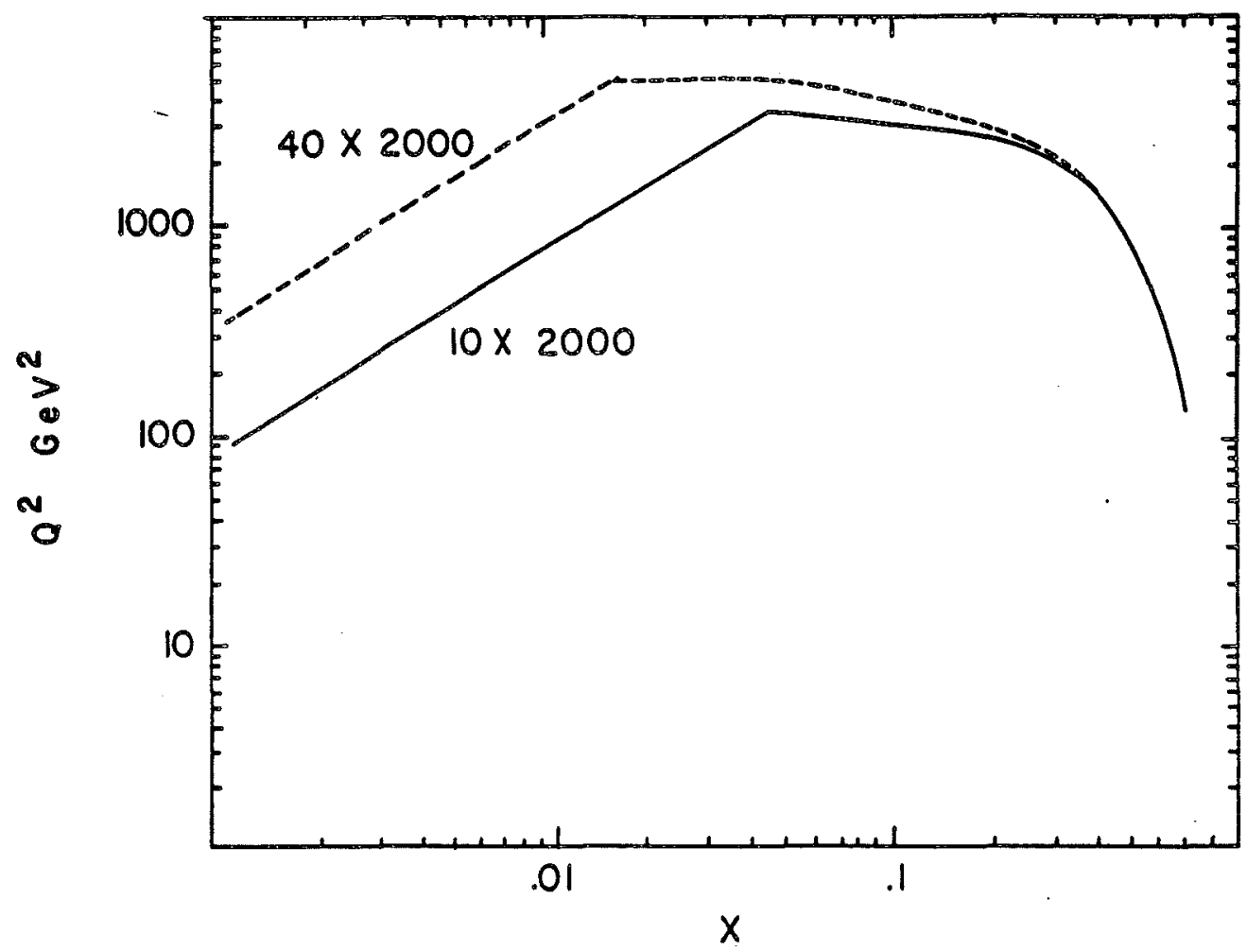

Fig. II-18. The $Q^{2}-x$ contours with bin sizes $d x / x=.1$ and $d^{2} / Q^{2}=.1$ and yielding per bin greater than 100 events/yr. in a 1 yr. run with integrated luminosity $5 \times 10^{38} \mathrm{~cm}^{-2}$.

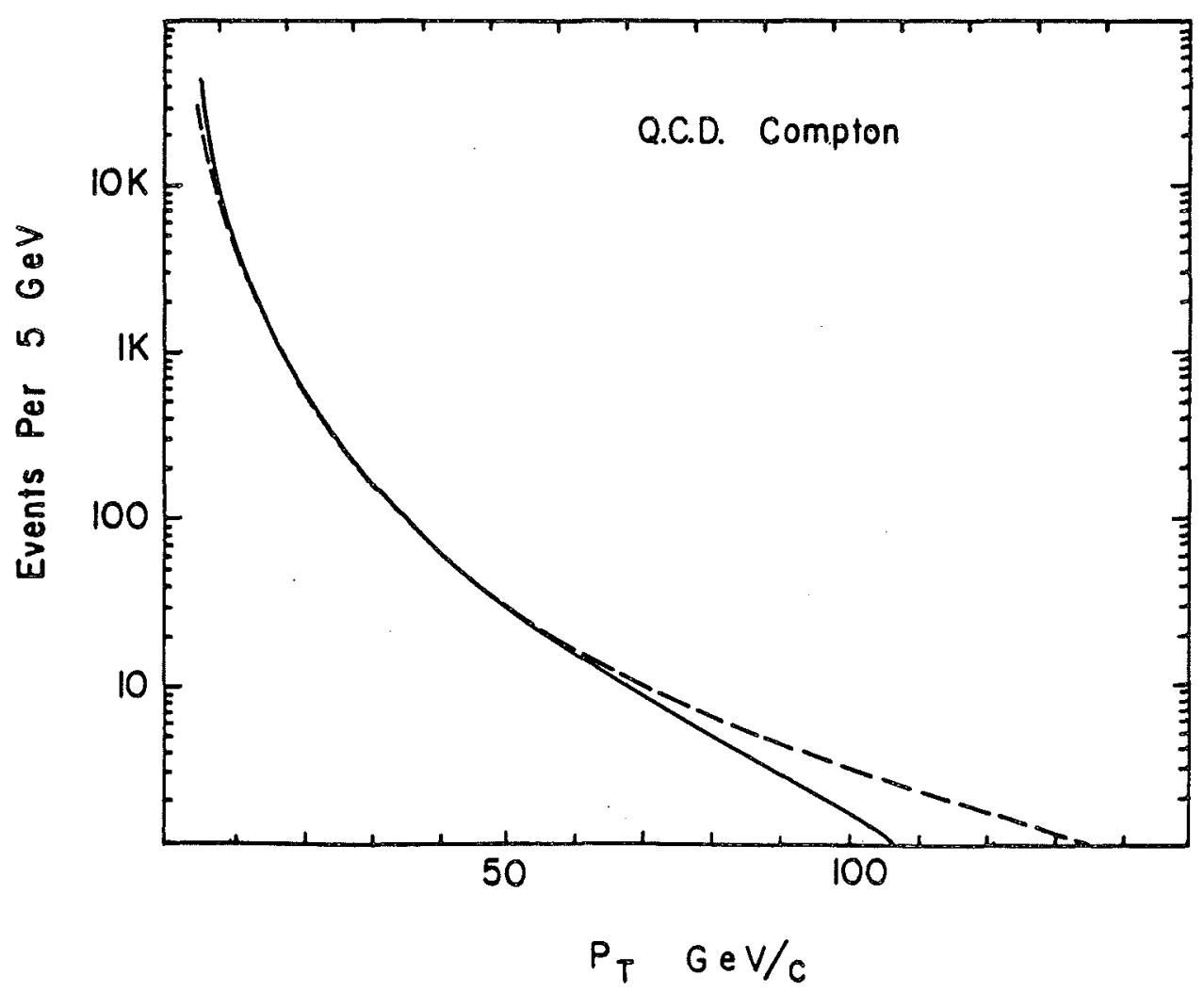

F1g. II-19. Expected yield of large tranverse momentum events anticipated in the QCD Compton process $\gamma+q \rightarrow g+q$ for a run of $\int \mathrm{d} t \mathcal{L}=5 \times 10^{38} \mathrm{~cm}^{-2}$ in $10 \mathrm{GeV}$ on $2 \mathrm{TeV}$ ep collisions. 
The study of the high-energy QCD Compton process depicted in Fig. II-19 provides a method to study gluon fragmentation. This process, where a high-energy photon scatters off a quark in the proton creating a wide angle gluon jet and a wide angle quark jet, is essentially the inverse of one of the mechanisms thought responsible for direct photon production in hadron-hadron collisions. In the kinematic regime where the final quark and gluon jet emerge with a large $p_{\text {f }}$ (say $\mathrm{p}_{t}>15 \mathrm{GeV}$ ), the cross section calculation should be fairly reliable, and hence measurements of the process will serve as powerful tests of QCD. The ratio of the QCD Compton cross section over the QED Compton cross section (which would have a high $p_{t}$ photon against a quark jet) serves as a direct and independent measurement of $\alpha$. Correlations are expected between the plane defined by the quark and giuon jets and the polarization vector of the virtual photon. These correlations serve to directly test the form of the quark gluon coupling. For example, this angular correlation is expected to have the opposite sign from the analogous correlation predicted for the QED Compton process.

The QCD Compton process being a low $Q^{2}$ process with two $h$ igh $p_{t}$ jets will produce events which can easily be experimentaliy distinguished from normal neutral current events consisting of a single large $p_{t}$ jet and a large $Q^{2}$ scattered electron. However, the light-quark photon-gluon fusion contribution produces a background with the same general topology as the QCD Compton process. It is possible to suppress the photon-gluon fusion process relative to the $Q C D$ Compton process by demanding that the parton within the incident proton which collides with the virtual photon has a large fraction of the incident proton momentum. A lower cut on parton energy fraction suppresses the gluon initiated process relative to the quark initiated process since gluons tend to carry a much smaller fraction of the proton momentum than the valence quarks. Energy balance can be used to find $\xi$, the fraction of momentum of the incident proton carried by the parton which collided with the virtual photon for a given two-jet event. The cut $\xi>0.6$ reduces the photon-gluon background to about $10 \%$.

Virtual photoproduction processes provide an excellent source for heavy quark production. For example, the expected top quark yield as a function of quark mass for the assumption of vector meson dominance is shown in Fig. II-20. A minimum electron tagging angle of $3^{\circ}$ is used and the photoproduction cross section is scaled by $\left(\mathrm{m}_{\mathrm{c}} / \mathrm{m}_{t}\right)^{2}$ from the known charm photoproduction cross section. Finally, a nigh $q^{2}$ cutoff of the vector meson form factor is included. The virtual photoproduction rates for $D^{\circ}, B, t \quad(m=20 \mathrm{GeV})$ and $t(m=50 \mathrm{GeV})$ are summarized in Table II-15. It should be added that these events are easy to recognize.

Finally, as shown in Fig. II-21, the photon-gluon fission process allows for the production of 100 events for top quark masses up to $80 \mathrm{GeV}$ in Phase I and $100 \mathrm{GeV}$ in Phase II. 


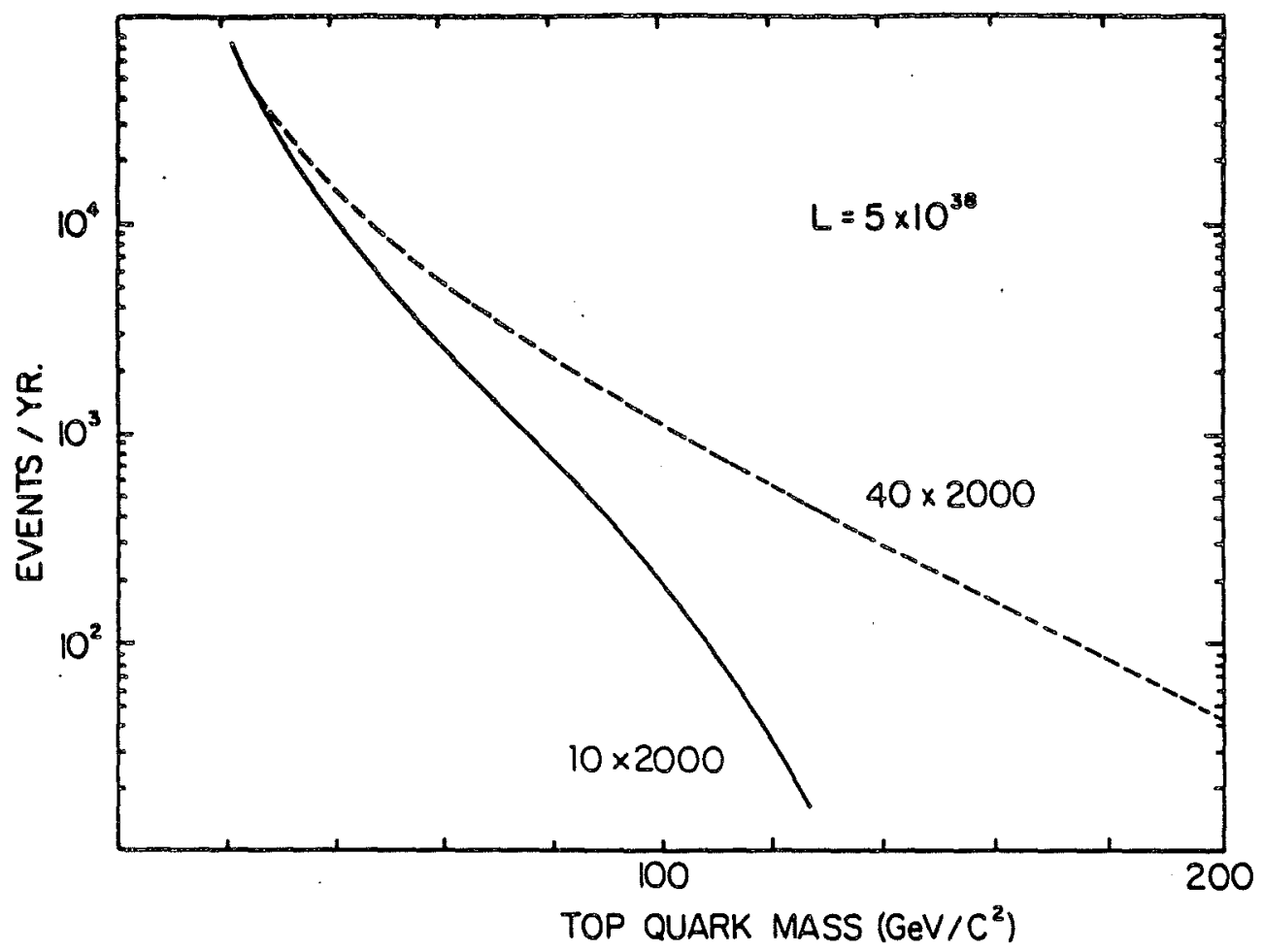

F1g. II-20. Yield of top quarks anticipated in ep collisions for an integrated luminosity of $\int \mathrm{dt} \mathscr{L}=5 \times 10^{38} \mathrm{~cm}^{-2}$.



F1g. II-21. Top quark pair production by photon-gluon fusion for $\int \mathrm{d} f \mathcal{L}=5 \times 10^{38} \mathrm{~cm}^{-2}$. 


\section{Standard Physics: Electroweak Pnenomena}

The asymmetry, $A$, in low $Q^{2}$ electron proton scattering observed at SLAC gave the first compelling evidence for the existence of weak and electromagnetic interferences in neutral current processes.

$$
\begin{aligned}
A= & \frac{\sigma_{e-}-\sigma_{e+}}{\sigma_{e-}+\sigma_{e+}}=\frac{2 \sqrt{2} G_{F}}{e^{2}} \frac{Q^{2}}{1+Q^{2} / m^{2}} \times \\
& \frac{1-(1-y)^{2}}{1+(1-y)^{2}} \frac{x F_{3}\left(x, Q^{2}\right)}{F_{2}{ }^{e m}\left(x, Q^{2}\right)} .
\end{aligned}
$$

This effect should reach $25 \%$ in the Phase $I$ ep collider. The major contribution of ep scattering for the neutral currents will be as a test of the standard model in the extreme spacelike region. Deviations from the expected $Q^{2}$ dependence of tire asymetry parameter $A$ could signify the presence of additional neatral vector bosons more massive than the $\mathrm{Z}^{\circ}$.

In the normal charged current process, an electron will scatter from a quark via a $W$ exchange leaving a neutrino (which will escape detection) and a wide angle current jet in the final state. The evolution of the charged current propagator allows a measurement of the mass of the $W$ to within $\pm 4 \mathrm{GeV}$ even with a $20 \%$ uncertainty in the ep Iuminosity.

By fitting the shape of the $W$ propagator, we will be sensitive to the possible effects of nultiple W's. The mass and coupling of the standard $W$ will have been determined from the Sp̄ps collider experiments. ijsing these as inputs to the fit of the effective charged-current propagator determined in the ep collider, the presence of a second $W$ can be determined up to a mass of $400 \mathrm{GeV}$.

\section{Extensions of the Standard Model}

We have seen that the $p \bar{p}$ collider at $D C$ will be sensitive to a second $W$ up to a mass $\sim 1200 \mathrm{GeV}$. In extensions of the standard model, one very appealing idea is that parity is restored at sufficiently high energies and therefore a ' $N$ ' wich couples to right-handed electrons would exist. If such $a W^{\prime}$ is found, the eo sollider could be invaluable in determining the handedness of $w^{*}$. If we assume that the right-handed W couples to right-handed leptons and quarks in a fashion analogous to the jert-handed couplings of the standard $w$ and that the right-handed $v_{p}$ is sufficientily ligit to give no additional kinematic suppression to these processes, then we can search for the presence of a right-handed charged current by looking for a $Q^{2}$ dependence in $R_{p}$ (the ratio of charged current events for right-handed polarized electrons to chargei 
current events for left-handed polarized electrons). The limit accessible to the ep Eacility in Phase $I$ is $600 \mathrm{GeV}$. We may alternately ask, to what level would the ep collider be sensitive to the existence of the neutral heavy lepton which is the final leptonic state in 2 right-handed charged current coupling. If we assume that the right-handed charged current coupling is identical in strength to the left-handed coupling, as shown in Fig. II-22, we would be sensitive to masses up to $200 \mathrm{GeV}$ in Phase I and $400 \mathrm{GeV}$ in Phase II.

\section{Other Possibilities}

\section{a) Technicolor}

Models winich construct quarks and leptons from elementary constituents or in which there is a new strong interaction predict the existence of leptoquarks with masses possibly in the vicinity of hundreds of $\mathrm{GeV}$ and widths which might be narrow. An ep collider offers unique advantages in searching for the existence of massive leptoguarks and leptogluons since they can be produced directly in the s-channel, and will produce peaks in the neutral current structure functions at an $\mathrm{x}=\mathrm{M}^{2} / \mathrm{s}$. Production of scalar leptoquarks is expected to be very low owing to the nature of their coupling. Vector leptoquark yields might be copious, however, if the lepton-quark interaction scale is on the order of $1 \mathrm{TeV}$. Figure III-23 gives the expected rates for our standard ep run. It assumes the following production cross sections.

$$
\begin{aligned}
& \sigma=\left.\frac{4 \pi^{2} \alpha_{L Q}}{M_{L Q}{ }^{2}} x u(x) \quad\right|_{x=\frac{M_{L Q}}{s}}{ }^{2} \\
& \sigma=4 \pi^{2} \alpha_{s} \frac{M_{L g}{ }^{2}}{\Lambda^{4}} x g(x) \quad \mid \begin{array}{l}
x=\frac{M_{L g}^{2}}{s} .
\end{array}
\end{aligned}
$$

\section{b) Supersymmetry}

In models with supersymmetry at the TeV scale each of the usual particles has a partner with spin differing by one half and a mass below a few hundred GeV. Although the pp collider will in general be more sensitive to these superpartners, an ep collider would produce 100 squark pairs/yr for masses up to $55 \mathrm{GeV}$ in Phase $I$ and $85 \mathrm{GeV}$ in Phase II. The production rate for squark pairs for an integrated luminosity of $5 \times 10^{38}$ is shown in Fig. II-24.

C) Quark Substructure and Compositeness

Other modifications to the standard model which could appear in ep interactions would be the observation of quark substructure. The 


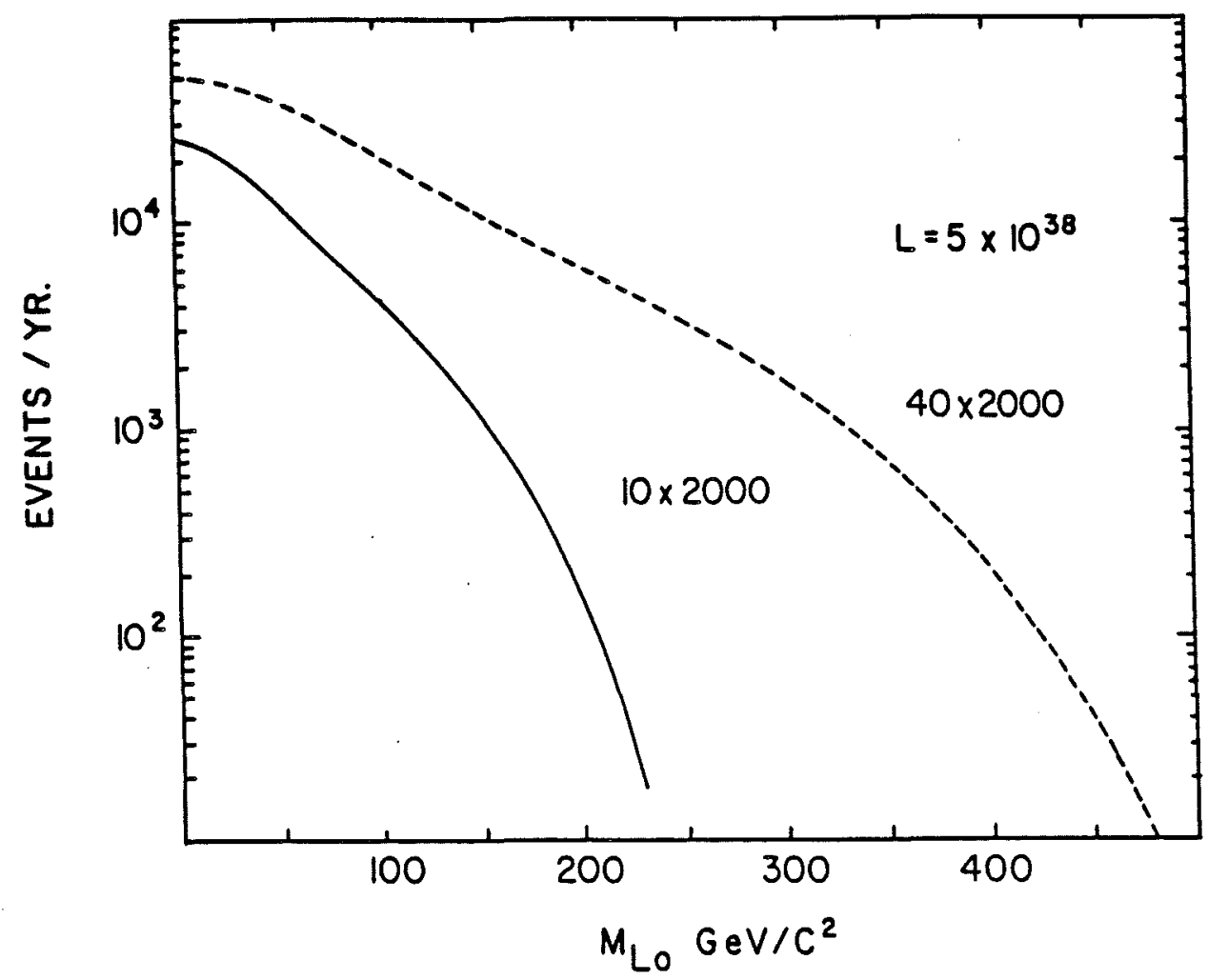

F1g. II-22. Sensitivity of the ep collider to neutral heavy leptons coupled to the electron with a right-handed interaction of universal Fermi strength.

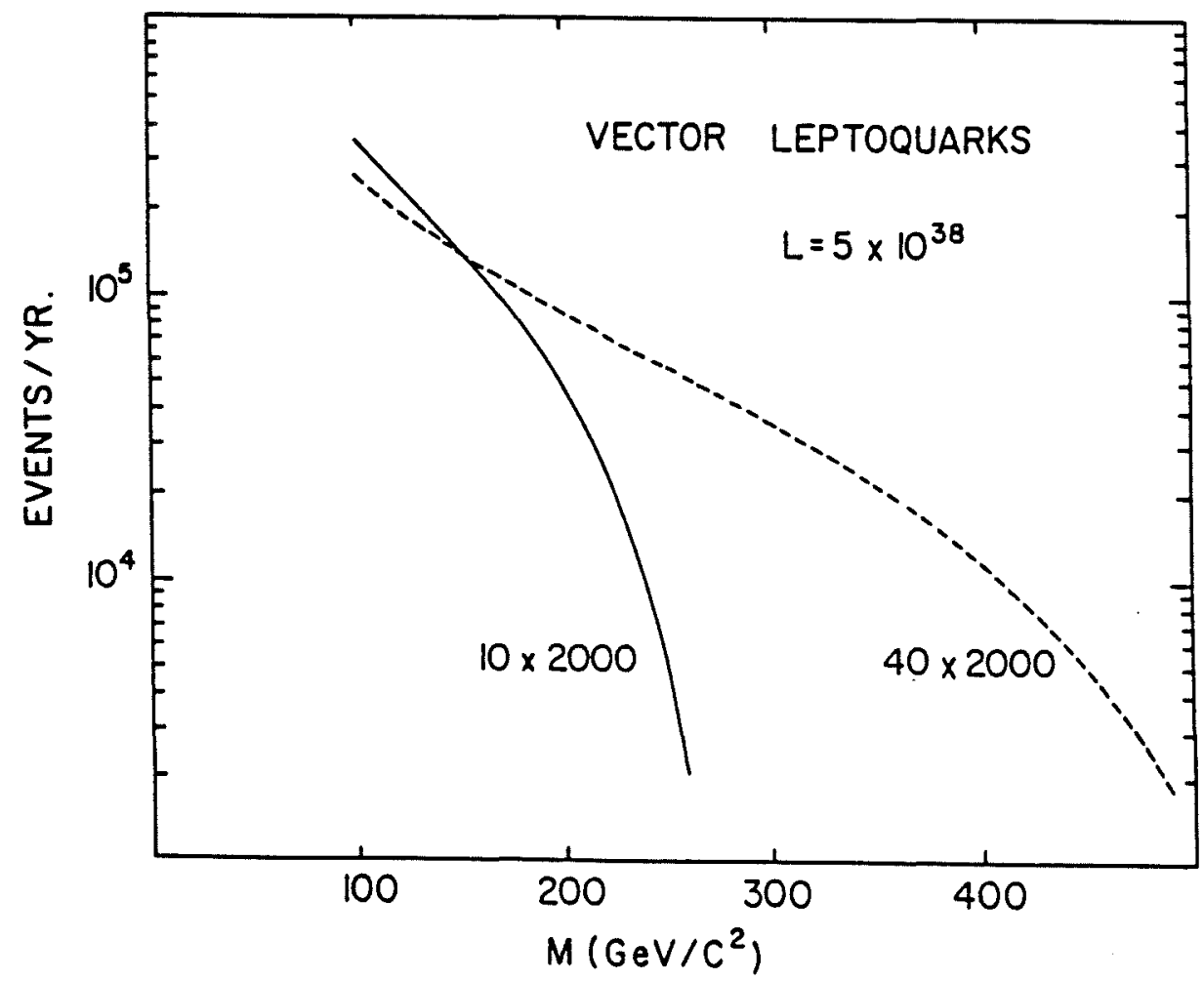

Fig. II-23. Yleld of vector leptoquarks in a run of $\int \mathrm{dt} \mathcal{L}=5 \times 10^{38} \mathrm{~cm}^{-2}$ in ep colliders. 
electron-proton collider will allow measurements of the nucleon structure function up to $\mathrm{Q}^{2}=10,000 \mathrm{GeV}$ at $\mathrm{s}=8 \times 10^{4} \mathrm{GeV}^{2}$ and searches for power law contributions to scale breaking at mass scales up to $500 \mathrm{GeV}$. Such power law contributions could be an indication of quark substructure. If quark substructure is present, it should manifest itself through the existence of events which look dramatically different from ordinary ep events.

If quarks and leptons are constructed from similar building blocks, one can anticipate new interactions where quark-lepton scattering occurs by constituent exchange rather than by photon exchange. Such a contact interaction will interfere with the normal neutral current amplitude to create a deviation which is approximately linear in $\mathrm{q}^{2}$ :

$$
\frac{\mathrm{d} \sigma / d Q^{2}}{d \sigma / d Q^{2}}=1 \pm \frac{A^{-1 / 2} Q^{2}}{\Lambda^{2}}
$$

where $\Lambda$ is the compositeness scale of quarks and leptons and $A$ is of order one. Figures II-25 and II-26 show the ratio of the yield of neutral current events with $\mathrm{q}^{2} / \mathrm{q}_{0}{ }^{2}$ when a contact term is present over the yield of neutral current events when a contact term is absent. The high-energy ep machine allows a considerable extension in $\mathrm{q}^{2}$. Figure II-27 compares the statistical significance of the contact effect as a function of the compositeness scale $\alpha \Lambda^{2}$ in units of $10^{5} \mathrm{GeV}^{2}$. The vertical coordinate is in standard deviations.

\section{Summary}

We summarize in Table II-16 the physics capabilities of an ep collider operating at $s=8 \times 10^{4} \mathrm{GeV}^{2}$ with an integrated luminosity of $5 \times 10^{38} \mathrm{~cm}^{-2}$. 


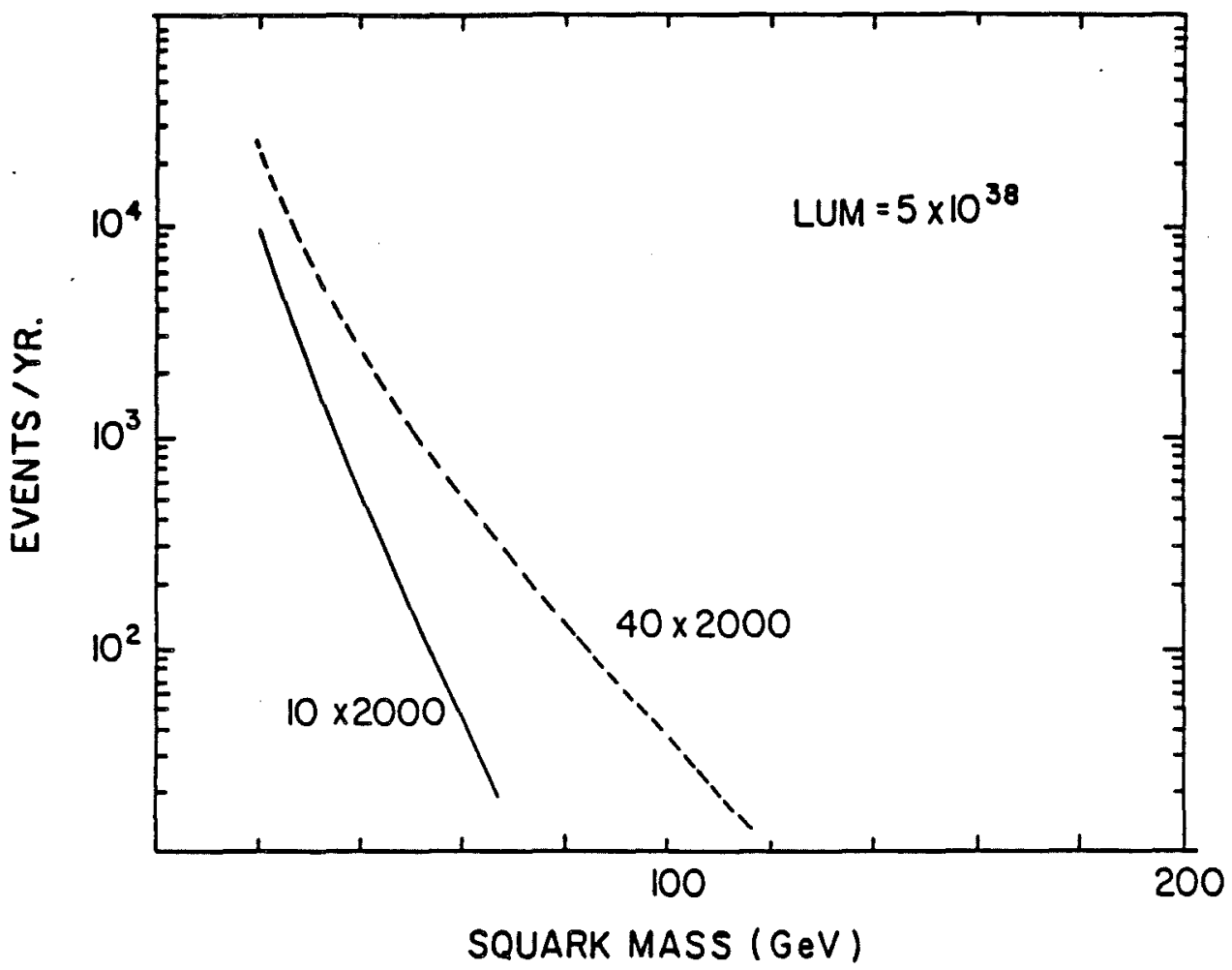

Fig. II-24, Expected yield of squark pairs with integrated luminosity $5 \times 10^{38} \mathrm{~cm}^{-2}$.

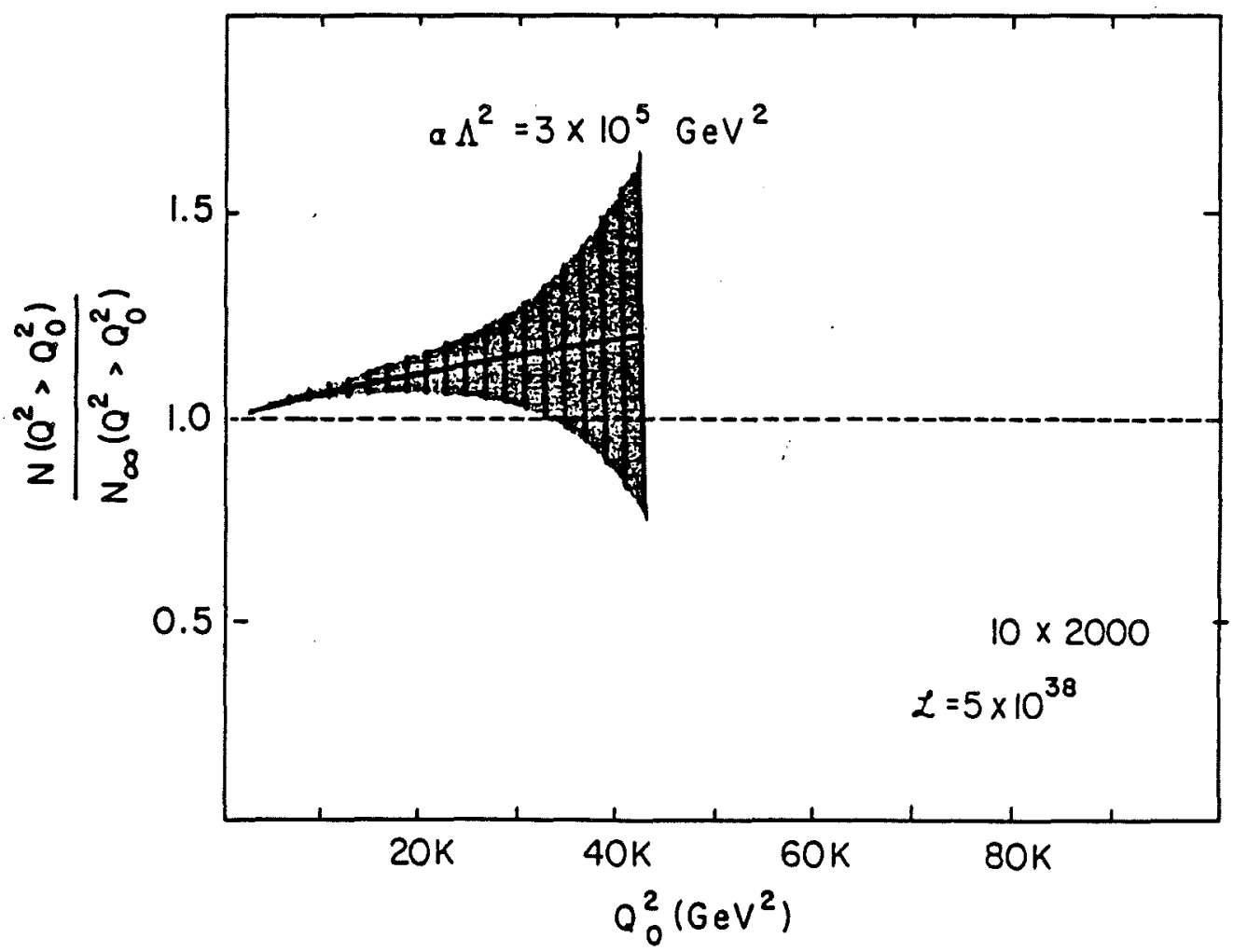

Fig. II-25. Ratio of the yield of neutral current events with $Q^{2}>Q_{0}{ }^{2}$ in the presence of a contact interaction to those expected in the conventional picture, for the $-10 \mathrm{GeV}$ on $2 \mathrm{TeV}$ ep collider. The error band is calculated for a run of integrated luminosity $\int \mathrm{dt} \mathscr{R}=5 \mathrm{x} 10^{38} \mathrm{~cm}^{-2}$. 
Table II-15. Virtual Photoproduction of Heavy Quark States

\section{Assumed Cross Section}

1) $\gamma^{*} \rightarrow D^{\circ} \bar{D}^{\circ}$

2) $\gamma^{*}+B \bar{B}$

3) $r^{*}+t \bar{t}$

( $20 \mathrm{GeV}$ top)

4) $\gamma+t \bar{t}$ ( $50 \mathrm{GeV}$ top)
$500 \mathrm{nb}\left(1-\frac{12 \mathrm{GeV}}{v}\right)$

$27 \mathrm{nb}\left(1-\frac{68 \mathrm{GeV}}{v}\right)$

$7 \mathrm{ab}\left(1-\frac{893 \mathrm{GeV}}{v}\right)$

$1.2 \mathrm{nb}\left(1-\frac{5431 \mathrm{GeV}}{v}\right)$

\section{Expected Iield *}

$1.0 \times 10^{7}$

$7.4 \times 10^{5}$

$1.5 \times 10^{5}$

1. $1 \times 10^{4}$

*Yield for an integrated Iuminosity of $5 \times 10^{38} \mathrm{~cm}^{-2} \quad 10$ on $2000 \mathrm{GeV}$ ep collisions.

Table II-16. Sensitivity of various processes for $s=8 \times 10^{4} \mathrm{GeV}^{2}$ and $\int \mathrm{dt} \mathcal{L}=5 \times 10^{38} \mathrm{~cm}^{-2}$

\section{Process}

QCD tests

wi width

W

Right-handed $W$

Top quark production

$\left(u_{t}=50 \mathrm{GeV}\right)$

Neutral Heavy Lepton

Vector Leptoquark

$$
\left(m_{L \vec{Q}}=250 \mathrm{GeV}\right)
$$

Squark production

$(100 \mathrm{ev} / \mathrm{yr})$

Quark substructure

Composite eq interaction
Limit

$Q_{\max }^{2} \simeq 10,000 \mathrm{GeV}^{2}$

$\Delta \mathrm{M}_{W}=4 \mathrm{GeV}$

$m_{W^{\prime}}=400 \mathrm{GeV}$

$\mathrm{m}_{\mathrm{W}}=600 \mathrm{GeV}$

6,000 events/yr

$m_{L} 0=200 \mathrm{GeV}$

4,000 events/yr

$55 \mathrm{GeV}$

$4.0 \times 10^{-17} \mathrm{~cm}$

$\Lambda>6 \mathrm{TeV}$ 


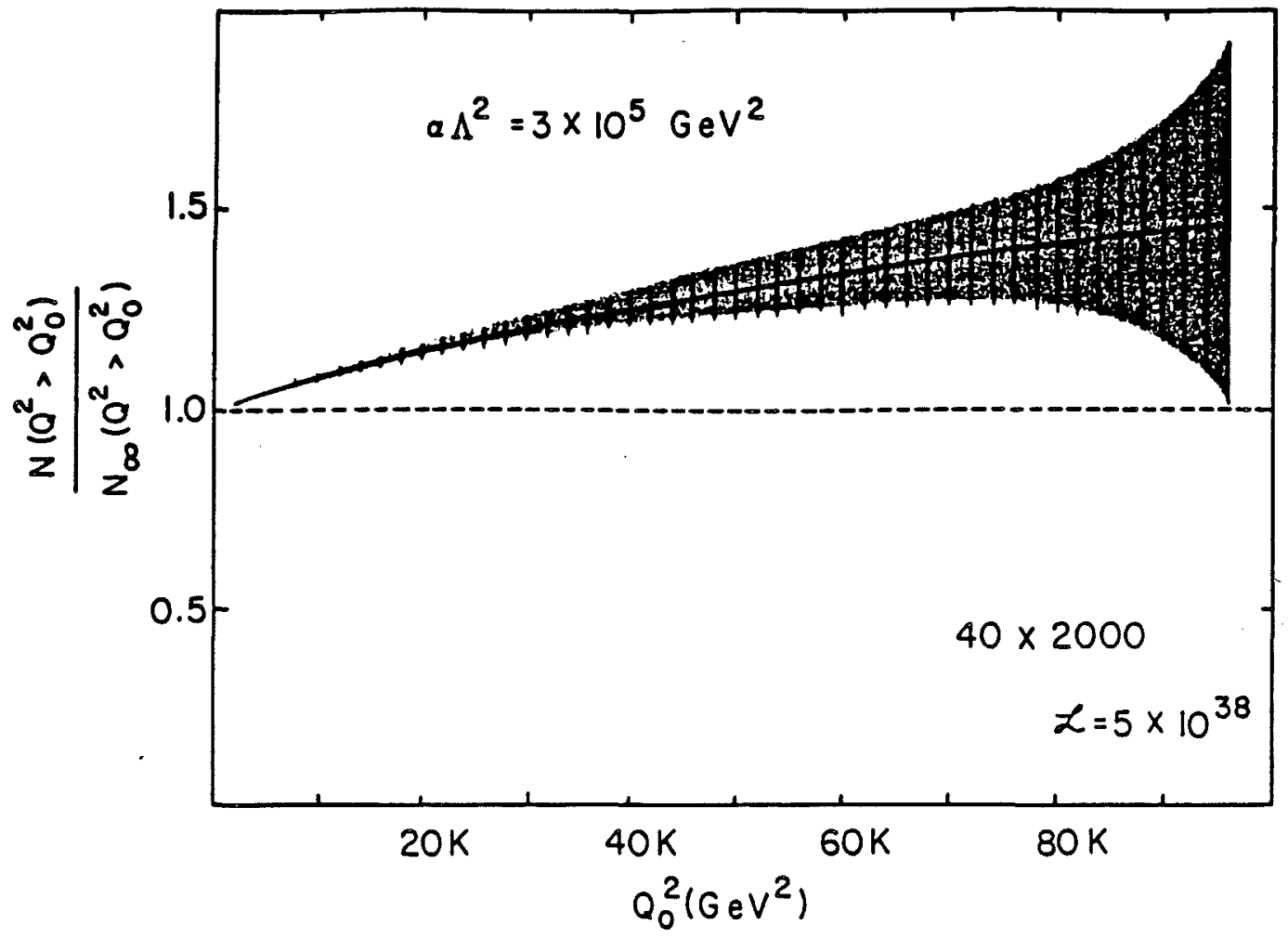

Fig. II-26. Ratio of the yield of neutral current events with $Q^{2}>Q_{0}{ }^{2}$ in the presence of a contact interaction to those expected in the conventional picture, for the $40 \mathrm{GeV}$ on $2 \mathrm{TeV}$ collisions. The error band is calculated for a run of integrated luminosity $\int \mathrm{d} t \mathcal{L}=5 \times 10^{38} \mathrm{~cm}^{-2}$.

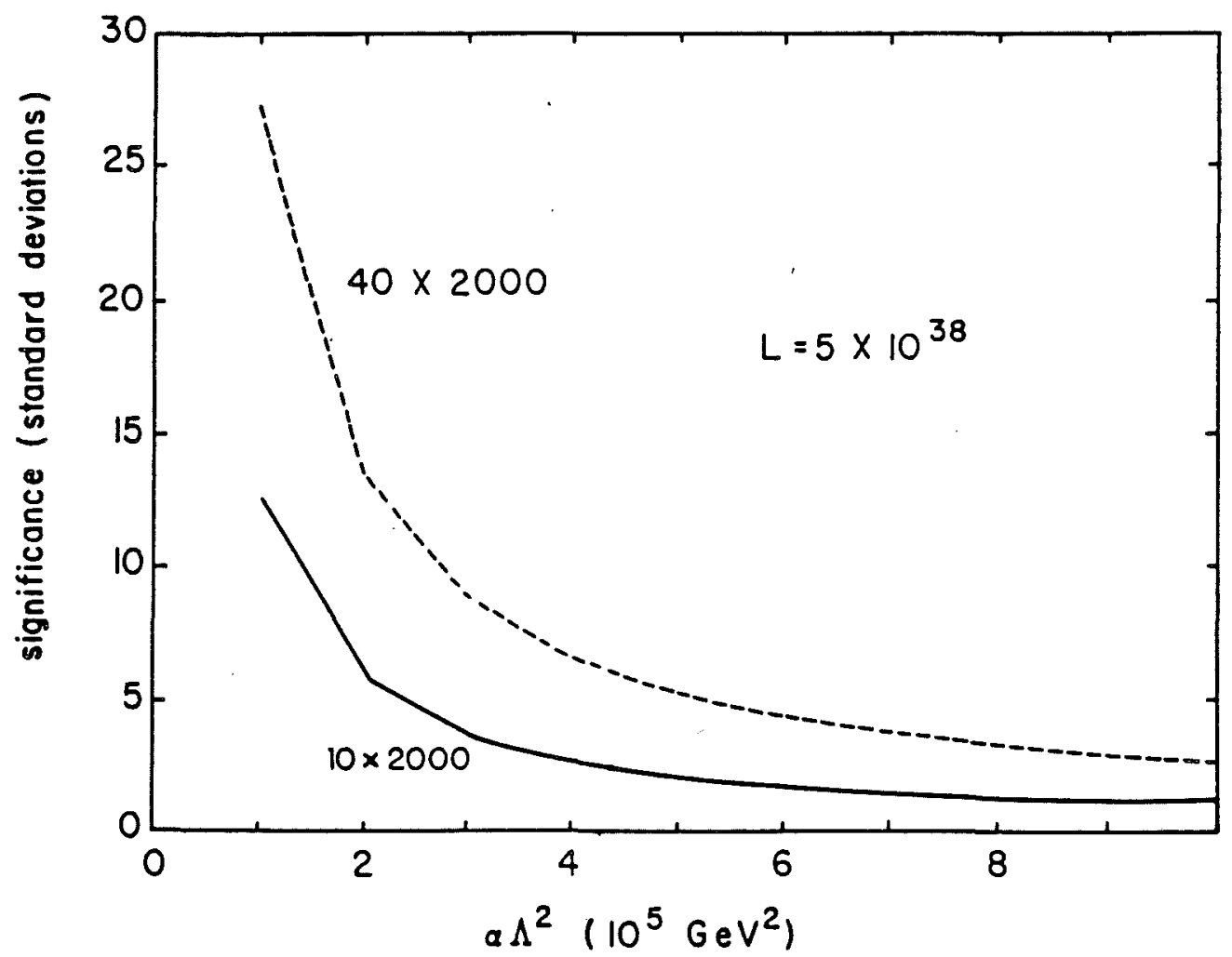

F18. II-27. Statistical significance of the contact term's effect upon neutral current cross sections as a function of the compositeness scale for a run of $\int \mathrm{dt} \mathscr{L}=5 \times 10^{38} \mathrm{~cm}^{-2}$ in ep colliders. 


\section{THE PROTON-ANTIPROTON COLLIDER}

\section{A. Design goals and constraints}

We have adopted the following goals and constraints:

1. The size of the ring should approach the largest that can be reasonably and conveniently contained in the Fermilab site. This provides the facility with optimal growth potential, either in the direction of higher energy or higher luminosity (e.g., pp collisions). The largest circle that can be drawn on the Fermilab site is about 2.5 $\mathrm{km}$ in radius. We have chosen a somewhat smaller radius of approximately $2 \mathrm{~km}$ for a variety of reasons. One is cost: $a 2 \mathrm{~km}$ ring is already close to the cost guidelines suggested by the Trilling subpanel for an "intermediate-range" dedicated collider facility. Another is geography: the most practical location for extraction of the beam from the Tevatron is at long straight section $E$. Then a natural axis of symmetry for the collider ring is a line from EO through the center of the Tevatron ring. If the ring even approximately respects this axis, a limitation of approximately $2 \mathrm{~km}$ radius follows. A truly site-filling ring, in addition, has greater problems with regard to the topography and geology of the land and to radiation-easement requirements. The layout of the rings on the Fermilab site is shown in Fig. I- 1 .

2. To minimize R\&D costs and to allow the start of construction (and hence the availability of this collider facility) to be as early as possible, we assume that Tevatron magnets will be used. The length of dipoles is increased from $6.1 \mathrm{~m}$ to $7.75 \mathrm{~m}$, and the length of quadrupoles from $1.7 \mathrm{~m}$ to $2.5 \mathrm{~m}$. Neither modification requires any change in the design concept, and the need for significant research and development on the superconducting magnets is minimal. The tooling and fixtures can be accommodated to the changes in lengths, and are therefore available for the fabrication of these magnets. Likewise, the cryogenic and power supply system design can be largely taken over from that of the Saver. With superconducting cable of quality equal to or better than the latest batch of Tevatron conductors, we can expect significant increase in the attainable dipole field. A value of $4.65 \mathrm{~T}$ is used in this design, and a further increase may be possible. A ring radius of $2 \mathrm{~km}$ implies an energy of about $2 \mathrm{TeV}$ per beam, or a total collision energy of $4 \mathrm{TeV}$. With 1-TeV injection, the 3-in. diameter aperture of the Tevatron is quite large for this application and is beneficial to the performance of the collider.

3. To maximize luminosity, we want to inject many bunches per beam. In addition, because of the large magnet aperture, the thresholds for many of the coupled bunch instabilities of the beams are quite high. on the other hand, bean-beam interaction effects will be excessive if the beams are allowed to collide over the entire circumference. To keep the beams separated except at the locations of the experimental 


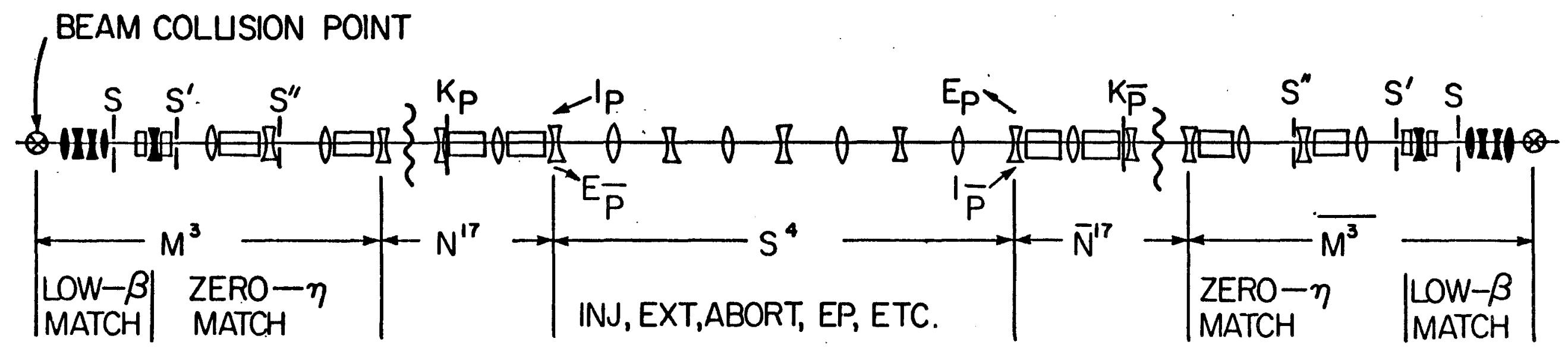

Fig. III-1 A Quadrant of the Dedicated Collider 
detectors, closed-orbit excursions are induced on the two oppositely charged beams by electrostatic separators. To take advantage of the maximum separation, we choose only one beam bunch per betatron wavelength. The desire to have a large number of bunches per beam therefore suggests a large betatron oscillation tune, that is, a strong-focusing lattice. Again, in the presence of susin distorted design orbits, the relatively large aperture of the Tevatron magnets presents a distinct advantage. On the other hand, this beam-separation scheme makes rather stringent demands on the design of the lattice insertion for the straight section for colliding beams, and on careful, independent control of the parameters of proton and antiproton beams. Preliminary estimates of these effects indicate that they will be manageable. Experience obtained from the operation of the Saver and SPS will provide very direct information for the design of the Dedicated Collider.

4. There are a number of standard constraints on the design of the collider ring originating from the use of superconducting magnets. The total beam current should be small to reduce the heating by image currents and by stray beam. High-efficiency beam-abort systems must be provided to avoid beam quenching of the magnets in case of an equipment failure or other emergencies. Mutually orthogonal $r f$ systems must be provided for the two beams to shift the phases of the beams relative to the injection point, the experimental detector, and to each other. These will be discussed in detail below and in Chapter IV in connection with the design of the component systems.

\section{B. The Injector}

One of the most attractive features of the proposed collider is the availability of the injector. By the time the construction of the $D C$ is complete, we will have an operating injector for both $p$ and $\bar{p}$ and experience in its operation. By that time, the injector may well have exceeded its original design goals. Nevertineless, we assume here, on the basis of the existing Tevatron design, the following parameters for antiprotons:

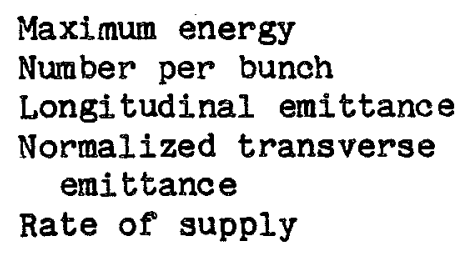

$$
\begin{gathered}
1 \mathrm{TeV} \\
10^{11} \\
3 \mathrm{eV}-\mathrm{sec} \\
24 \pi \mathrm{mm}-\mathrm{mrad} \\
1 \mathrm{bunch} / \mathrm{hr} \\
\left(10^{11} \mathrm{p} / \mathrm{hr}\right)
\end{gathered}
$$

The same parameters apply for protons except the rate of supply is much greater. 
In the present configuration, the transfer of beams from the Main Ring to the Tevatron is carried out in straight section $E$. To make this straight section available for beam transfer to the $D C$, we must move the Main Ring-Tevatron beam transfer to another straight section. Straight sections $A, C$, and $F$ are already fully occupied with accelerator functions, which leaves only $D$ and $B$ to consider. While the $D C$ is engaged in 4-TeV colliding-beams experiments, the Tevatron will be available for 1-TeV fixed target physics during more than $90 \%$ of the time. Thus the beam-extraction septum in straight section $D$ will be needed and hence the Main Ring-Tevatron beam transfer should be relocated in straight section $B$. $B$ is the long straight section where $1 \mathrm{TeV} \times 1 \mathrm{TeV}$ colliding beams will take place in the Tevatron I effort. This location is logistically proper because it is unlikely that with the DC operating, experiments with 2- $\mathrm{TeV}$ colliding beams in the Tevatron would continue.

The scheme for extracting both the $p$ and the $\bar{p}$ beams in the Tevatron at $1 \mathrm{TeV}$ from straight section $\mathrm{E}$ will be discussed below in connection with injection into the DC.

\section{Ring Lattice}

1. General description and parameters

The magnet lattice of the ring is composed of four identical symmetric sectors, each containing one low- $\beta$ collision region and a long but simple general-purpose straight section. The long straight sections are used for beam injection, extraction and abort, for ep collision regions, and for future extensions and additions. The low- $\beta$ collision regions and the long straight sections alternate at equal spacings. The lattice thus possesses four-fold periodicity.

For many beam manipulations, it is advantageous to operate the DC rf system at the same frequency as the Tevatron rf system. Thus, the $r f$ wavelength of the $D C$ is

$$
\lambda_{\mathrm{rf}}=\frac{2 \pi(1000 \mathrm{~m})}{1113}=5.65 \mathrm{~m} .
$$

The spacing between beam bunches is an integral multiple of $\lambda_{\text {f }}$ and beeause of the manner in which the beams are separated fy the electrostatic separators, the bunch separation should also be the betatron wave length in normal cells $\lambda_{\beta}$. It is convenient to choose an integral multiple that is a factor of the Tevatron harmonic number 1113. We choose 53 to give bunch spacing $=\lambda_{\beta}=53 \lambda_{\mathrm{rf}}=300 \mathrm{~m}$. With a 4-fold periodic lattice, it is convenient to have the number of bunches $N_{p}$ per beam be a multiple of 4 and we therefore choose $\mathrm{N}_{b}$ to be 44 . Altogether, we have a ring circumference $2 \pi R=13.2 \mathrm{~km}$ and $\mathrm{a}$ mean radius $\mathrm{R}=2.095 \mathrm{~km}$. 
We choose a $90^{\circ}$ separated-function FODO cell as the normal cell. Each cell will then have length $\lambda_{\beta} / 4=74.8 \mathrm{~m}$. The long utility straight section is formed by omitting the dipoles in 4 consecutive cells. In this way, both the amplitude function $B$ and the dispersion function $\eta$ are automatically matched across the long straight section. The collision region is specially matched for low $\beta$ and zero $n$. Two cell lengths on either side of the collision point are used for a simple zero- $\eta$ matching structure and one more cell length on either side is required for the low- $\beta$ matching. Denoting the matching structure on one side by $M^{3}$, we can then write the lattice for the entire ring as $\left(\mathrm{M}^{3} \mathrm{~N}^{17} \mathrm{~S}^{4} \mathrm{~N}^{17} \overrightarrow{\mathrm{M}}\right)^{4}$. Here $\mathrm{N}$ is a normal cell, $\mathrm{S}$ is a straight cell (cell with dipoles omitted) and $\vec{M}^{3}$ is $M^{3}$ transposed. Each normal half cell contains 4 dipoles and the matching structure $M^{3}$ contains 10 dipoles. The total number of dipoles is then $n_{B}=4 \times 34 \times 8+4 \times 20=1168$. A quadrant of the ring is shown in Fig. III-1. The lattice functions of a regular cell, one half of a long straight section (two straight cells), and a half matching section $\left(M^{3}\right)$ are shown in Figs. III-2a, III-2b, and III-2c respectively. The lattice parameters are summarized in Table III- 1.

Table III-1. Lattice Parameters of the DC

Normal cell $90^{\circ}$ separated function FODO cell

Half cell length $\ell$

$$
\begin{gathered}
37.4 m \\
\left(M^{3} N^{17} s^{4} N^{17} \overline{M^{3}}\right)^{4}
\end{gathered}
$$

Ring lattice structure

$$
\begin{aligned}
& N=\text { normal cell } \quad S=s t r a i g h t \text { cell } \\
& M^{3} M^{3}=\text { low-B zero-n matching section }
\end{aligned}
$$

Ring circumference $2 \pi R$

Ring radius $R$

Number of dipole $n_{B}$

Length of dipole $l_{B}$

Bending radius $\rho$

Rigidity of beam $B p$

Peak dipole field B

Bending angle per dipole $\theta$

Length of cell quadrupole $l_{Q}$

Peak gradient of cell quadrupole B'

$$
\begin{gathered}
13164.80 \mathrm{~m} \\
2095.24 \mathrm{~m} \\
1168 \\
7.75 \mathrm{~m} \\
1440.67 \mathrm{~m} \\
6.67 \times 10^{4} \mathrm{kG}-\mathrm{m} \\
46.3 \mathrm{~kg} \\
5.38 \mathrm{mrad} \\
2.5 \mathrm{~m} \\
\simeq 1035 \mathrm{~kg} / \mathrm{m}
\end{gathered}
$$









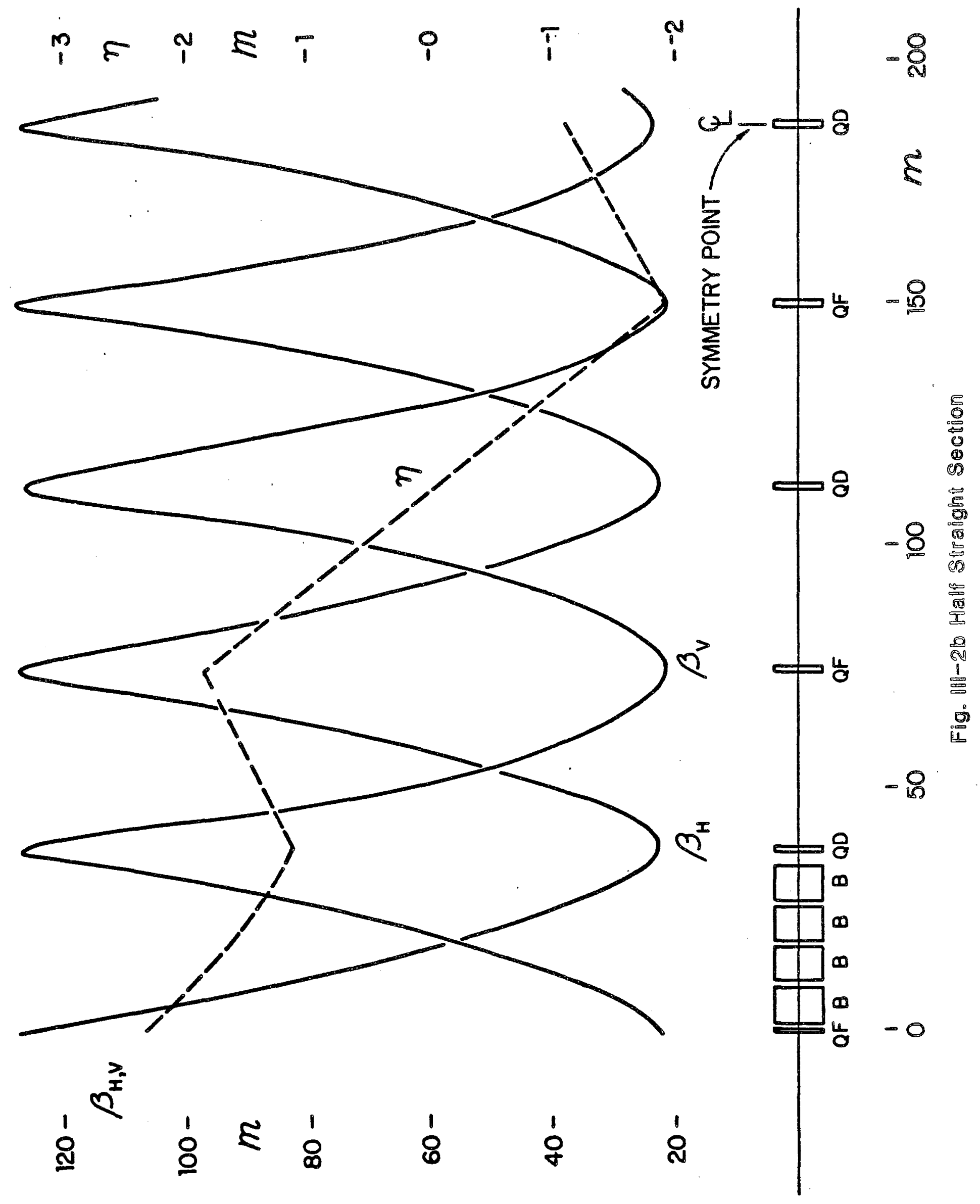




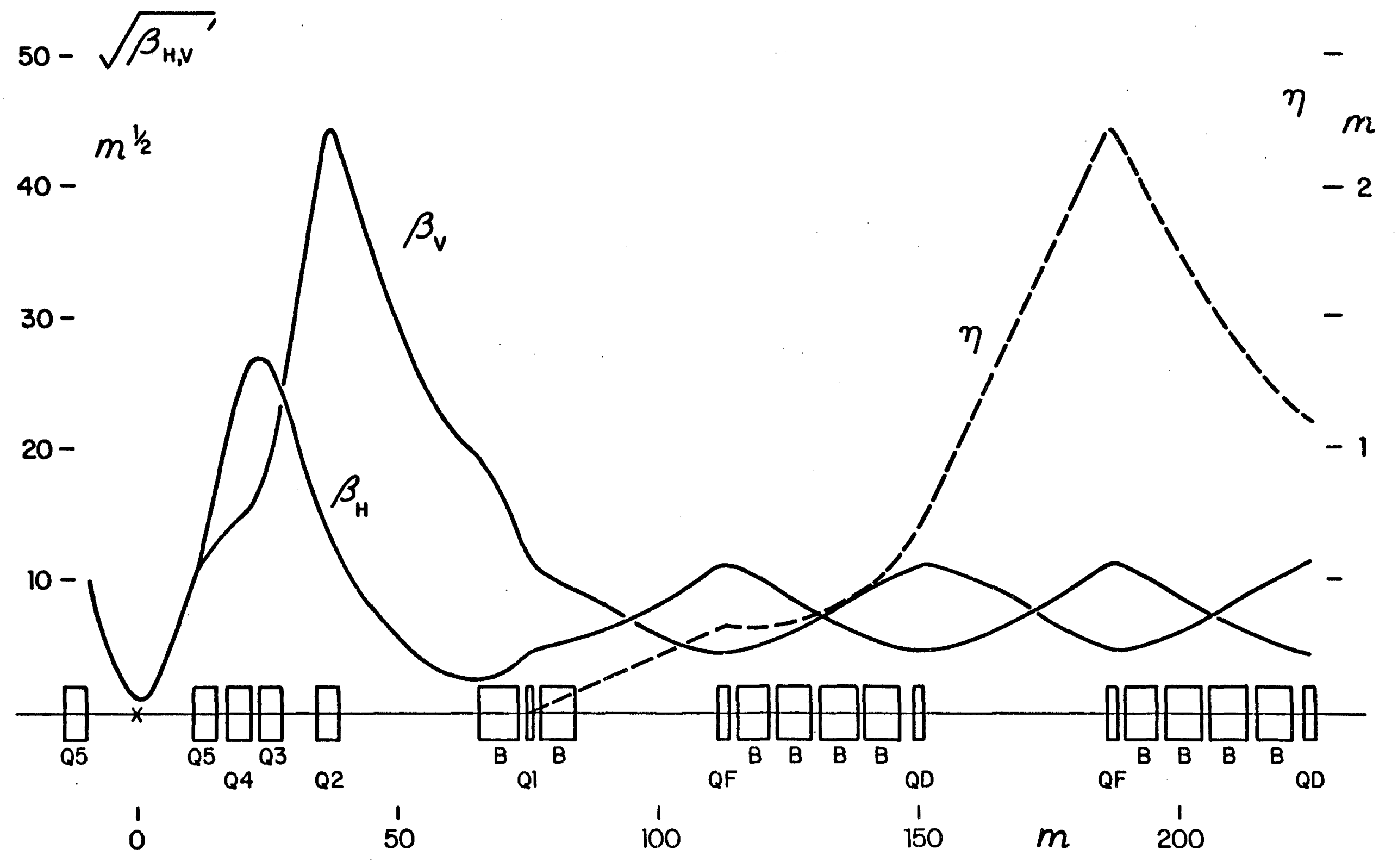

Fia. lll-2c Half Matching Section. 
(slightly different in $Q F$ and $Q D$ )

Max. normal $\beta, \beta_{\max }$
Min. normal $\beta, \beta_{\min }$
Max. normal $n, \eta_{\max }$
Min. normal $n, \eta_{\min }$
Transition energy $\gamma_{t}$

Phase advance per cell $\mu$

Phase advance in $\mathrm{M}^{3}$ and $\overline{\mathrm{M}}^{3} \mu_{\mathrm{m}}$

Tune $v$

Natural chromaticity $\xi$
$127 \mathrm{~m}$

$22.5 \mathrm{~m}$

$2.2 \mathrm{~m}$

$1.07 \mathrm{~m}$

37.2

\section{Horizontal Vertical}

The lattice parameters, including low- $\beta$ insertions and sextupole corrections, have been calculated with the computer codes SYNCH and PATRICIA.

\section{The low- $B$ zero-n insertion}

The special low- $\beta$ zero-n matching structure $M^{3}$ is shown in Fig. III-2c together with the $\beta_{\infty}$ and $n$-functions. For $90^{\circ}$ cells, several zero- $n$ matching schemes are available. The one we have chosen requires no special length dipoles or special strength quadrupoles. The low- $B$ matching consists of adjusting the strength of the end cell-quadrupole $Q_{1}$ and adding four separate quadrupoles $Q_{2}$ to $Q_{5}$ operating at polarities and strengths that suggest the action of a triplet. By independently adjusting the gradients of the 5 quadrupoles, one can vary $\beta$ at the collision point from approximately $5 \mathrm{~m}$ continuously down to approximately $1 \mathrm{~m}$. The parameters of the matching quadrupoles $Q_{1}$ to $Q_{5}$ for $\beta^{*}=1 \mathrm{~m}, \beta^{*}=3 \mathrm{~m}$, and $\beta^{*}=5 \mathrm{~m}$ are given in Table III-2. The operatioh of the electrostatic beam separators to keep the $p$ and $\bar{p}$ beams separated except at the collision points imposes additional requirements on the the matching structure $\mathrm{M}^{3}$. This will be discussed in the next section. 
Table III-2. Low-8 Parameters

\begin{tabular}{|c|c|c|c|c|}
\hline$\beta *$ & 1 & 3 & 5 & m \\
\hline$\nu_{\mathrm{H}}$ & 46.602 & 46.601 & 46.599 & \\
\hline$v_{v}$ & 44.402 & 44.399 & 44.401 & \\
\hline$\xi_{\mathrm{H}}$ & -92.1 & -69.1 & -68.5 & \\
\hline$\xi_{\mathrm{V}}$ & -118.7 & -67.6 & -57.1 & \\
\hline$B_{H}(\max )$ & 725 & 286 & 297 & m \\
\hline$\beta_{V}(\max )$ & 1925 & 478 & 129 & m \\
\hline $\begin{array}{c}\text { Quadrupole } \\
\qquad \begin{array}{c}Q_{F} \\
Q_{D} \\
Q_{1} \\
Q_{2}^{2} \\
Q_{3}^{3} \\
Q_{5}^{4}\end{array}\end{array}$ & $\begin{array}{l}0.0154 \\
0.0155 \\
0.0231 \\
0.0154 \\
0.0136 \\
0.0136 \\
0.0154\end{array}$ & $\begin{array}{l}\text { parameter } \\
0.0157 \\
0.0155 \\
0.0022 \\
0.0137 \\
0.0140 \\
0.0129 \\
0.0180\end{array}$ & $\begin{array}{c}\mathrm{K}=\mathrm{B}^{\prime} / \mathrm{Bp} \\
0.0158 \\
0.0154 \\
-0.0098 \\
0.0099 \\
0.0167 \\
0.0083 \\
0.0248\end{array}$ & $\begin{array}{l}m^{-2} \\
m^{-2} \\
m^{-2} \\
m^{-2} \\
m^{-2} \\
m^{-2} \\
m^{-2}\end{array}$ \\
\hline
\end{tabular}

\section{Beam separation}

The beam-beam interaction during the collision of a $p$ bunch and a $\bar{p}$ bunch is strong enough that if beams with 44 bunches each were allowed to collide over the entire circumference (hence 88 collisions per revolution), luminosity would be wasted and, in addition, degraded by beam-bean forces in a very short time. It is therefore important that the beams be kept apart except at locations where collisions are desired. To do this we must use electric fields, which exert opposite actions on protons and antiprotons.

The beams are separated yerticaly. At the location of high ${ }^{B} v_{v}$ in the matching insertions $\mathrm{M}^{3}$ and $\mathrm{M}^{3}$, an electrostatic separator $\mathrm{S}$ is placed to impart opposite deflections to the $p$ and $\bar{p}$ beams, as shown in Fig. III-3. These deflections translate to vertical displacements at $Q_{D}$ two cell lengths away from the collision point, where the bunches would collide again had it not been for the displacements induced by the separator. The phase advance from the separator $S$ to the first low- $\beta$ quadrupole $Q_{1}$ is small and amounts to only $4^{\circ}$. This is because of the very high $B$ value, $1900 \mathrm{~m}$, at $S$. Therefore, the phase advance from $S$ to $Q_{D}$ is essentially $90^{\circ}$. A second separator is placed at $S^{\prime}$, first, to deflect the beams futher and, second, to fine-adjust the phase advance. The beam bunches will pass one another at all locations every two cells 
beyond $Q_{B}$. At all these locations, the beam separations are identical to that at $\mathrm{B}_{\mathrm{D}}$.

The vertical rms beam half-width at $Q_{D}(\beta=127 \mathrm{~m})$ is $\sigma_{y} \equiv 0.5 \mathrm{~mm}$ at $2 \mathrm{TeV}$. The criterion for separation necessary to make the electromagnetic interaction between the two beams negligible has been studied and we have taken the separation to be $10 \sigma_{\mathrm{v}^{\circ}}$. To produce a beam separation of $\pm 5 \sigma_{\mathrm{v}}= \pm 2.5 \mathrm{~mm}$, the strength of the separator $S$, if alone at $\beta_{v}=1900 \mathrm{~m}$, should be $12 \mathrm{MV}$, which can be supplied with a field $\mathrm{E}=100 \mathrm{vV} / \mathrm{cm}$ and length $1.2 \mathrm{~m}$.

To obtain exact head-on collision at the next collision point it is essential to form exact point-to-point geometry between the extreme separators in the insertions $M^{3}$ and $\vec{M}$ of the quadrant $M^{3} N^{17} S^{4} N^{17} \vec{M}^{3}$. This is adjusted by an additional separator $S$ " placed next to $Q_{D}$. Both $S^{\prime}$ and $S^{\prime \prime}$ act as phase adjusters to trim the exact coincidence of the proton and the antiproton orbits in the collision regions.

It is expected that the beam separating operation will be delicate. We expect that the initial operations will be done with only a few bunches in each beam, for which beam separation will not be necessary. With all beam separators turned off and with the frequencies (phases) of the $p$ and $\bar{p}$ rf systems locked to each other, head-on collisions of beam bunches are automatically exact. The initial luminosity will be relatively low, of order $1-2 \times 10^{30} \mathrm{~cm}^{-2} \mathrm{sec}^{-1}$. Only as experience is gained will the number of beam bunches be increased, the beams separated, and the luminosity upgraded to above $10^{31} \mathrm{~cm}^{-2} \mathrm{sec}^{-1}$. We emphasize again that experience on the Saver and on separated-beams operation in existing $p \bar{p}$ colliders will be an effective aid in the design of the elements.

\section{Correction magnets}

As in all accelerators and colliders, field corrections are needed. The effects of field errors and their compensations have been studied in detail for the Tevatron and the experiences gained on the Tevatron will be directly applicable to the Dedicated Collider. The systems of correction magnets required are similar to those of the Tevatron with the exception that here we do not have a slow-extraction system, and hence no particles with large betatron oscillations. The whole system of correction magnets is described below.

\section{a. Steering dipole}

The closed-orbit distortions must be corrected at all energies. Individually controlled steering dipoles are placed next to each cell quadrupole, horizontal dipoles at focusing quadrupoles and vertical dipoles at defocusing quadrupoles. A minimum of two pairs (horizontal/vertical) of dipoles are needed for each insertion $M^{3}$ or $\overline{M^{3}}$. One pair is placed next to $Q_{5}$ and the other pair next to $Q_{2}$. The 


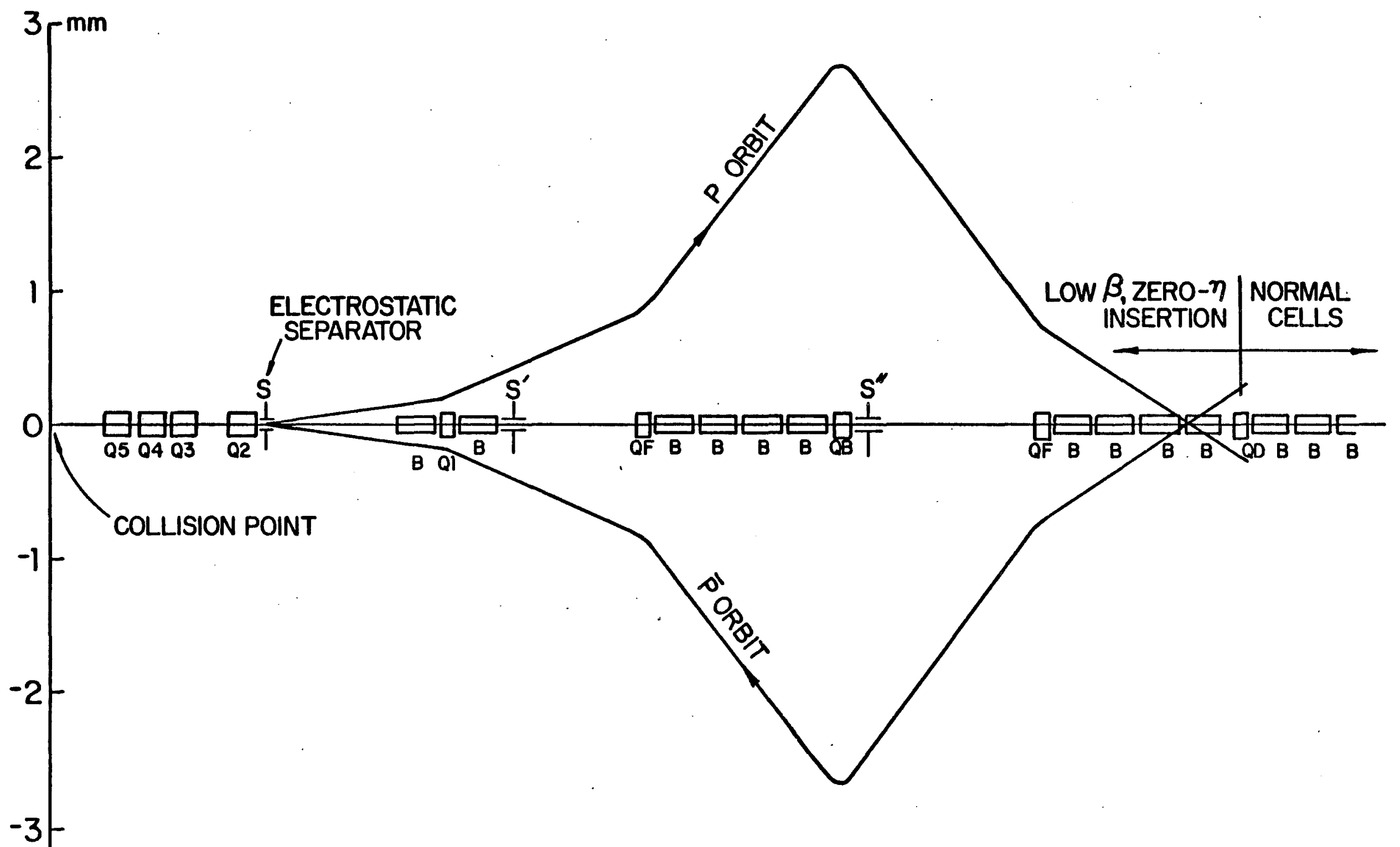

Fig. III-3 Elevation view of the separated $p$ and $\bar{p}$ orbits with electrostatic separator $s$ turned on, and $s^{\prime}$ and $s^{\prime \prime}$ off. 
required steering dipole strength is $170 \mathrm{kG}-i n .$, the same as in the Tevarron.

\section{b. Trim quadrupoles}

The trim quadrupoles may be used both to reduce the half-integer stopband width and to adjust the tunes. Trim quadrupoles will be located together with the steering dipoles at each cell quadrupole. trim quadrupoles are necessary in the matching insertions $\mathrm{M}^{3}$ and $\mathrm{M}^{3}$, because the low- $\beta$ quadrupoles are separately powered and individually adjustable. The trim quadrupoles need not be individually powered. They are connected and powered in 6 sets, one each for adjustment of $v_{H}$ and $v_{v}$ and two each for trimming out the $\nu_{y}=93 / 2$ and $\nu_{y}=89 / 2$ stopbands. Because of the existence of 4 collision regions, the trim-quadrupole strength required is 4 times that of the Tevatron, namely $B \cdot l=240 \mathrm{kG}$. These are strong, but feasible quadrupoles. Alternatively, a better solution might be to place special phase-adjust (tune-adjust) insertions in some of the long straight sections. Separately powering the normal-lattice quadrupoles is another option.

\section{c. Sextupoles}

The prineipal role of the sextupoles is to adjust the chromaticity. In this sense, they are not correction elements, but "normal" magneis. Although harmonic sextupoles are useful in compensating for excitation of the third-integer resonances $v_{y}=140 / 3$ and $v_{v}=133 / 3$, excitation prom error fields is expected to be small. Sextupoles are also packaged with the steering dipoles and the trim quadrupoles at the locations of cell quadrupoles. For chromaticity adjustment the sextupoles at the cell quadrupoles should be connected and powered in 2 sets to adjust the horizontal and the vertical chromaticities separately. To reduce the chromaticities to zero we need a strength of $\mathrm{B}$ ill $=-67 \mathrm{kG} /$ in for sextupoles next to focusing quadrupoles and a strength of $B^{\prime \prime l} l=$ $157 \mathrm{kG} / \mathrm{in}$ for those adjacent to defocusing quadrupoles. These are modest values. The design sextupoles will have strengin adjustable up to $\mathrm{B} \cdot \mathrm{ll}=200 \mathrm{~kg} / \mathrm{in}$. With chromaticities compensated to zero the tune variations across the full momentum spread of $\delta \mathrm{p} / \mathrm{p}= \pm 2 \times 10^{-4}$ of the beams are within $2 \times 10^{-4}$ for $\nu_{V}$ and $0.2 \times 10^{-4}$ for $v_{H}$.

\section{d. Octupoles}

Although we do not have to contain beams with large betatron oscillations, we do have beams that travel on off-center closed orbits. It may be necessary to adjust for the chromaticities of both on-center and of f-center orbits. The capability can be provided by the octupoles, which yield a sextupole field linearly dependent on the displacement. In the extreme, if one requires zero sextupole on the central orbit and the full sextupole strength of $B^{\prime} \cdot l=200 \mathrm{kG} / \mathrm{in}$ on an orbit 1 in. off center, one would need an octupole strength of $\mathrm{B}+1 \cdot l=200 \mathrm{kG} / \mathrm{in}$., But this extreme is not necessary and a strength half that value or 
$B \cdots l=100 \mathrm{kG} / \mathrm{in}^{2}$ should be adequate. Separate octupoles for individual low- $\beta$ quadrupoles are also planned.

\section{e. Skew multipoles}

Skew quadrupoles are needed only to remove linear coupling of the horizontal and the vertical oscillations. Approximately one skew quadrupole every 4 cells is adequate. These are the usual trim quadrupoles rotated $45^{\circ}$ about the beam axis.

With orbits separated vertically there will be skew quadrupole contributions from the chromaticity sextupoles. However, because of the 4-cell periodicity of the vertical orbit excursion the cancellation of these contributions is almost perfect. This can be seen from the orbit plot of Fig. III-3. The total residual skew quadrupole strength due to the slight phase shifts amounts to no more than $B \cdot l \backsim 1 / 2 \mathrm{kG}$ (rotated $45^{\circ}$ ) and is totally negligible.

Higher-order coupling resonances are all weak and correction is probably not necessary.

\section{Injection, Extraction and Abort \\ 1. Extraction from Tevatron}

Both the $p$ and $\bar{p}$ beams are extracted from straight section $E$ of the Tevatron, the $p$ beam clockwise and the $\bar{p}$ beam counterclockwise. As an injector for the DC, the Tevatron is operated with a regular lattice (no low- $\beta$ or high- $\beta$ insertions) at a tune of 19.4. The $p$ extraction channel starts at $D 50$ where $\beta_{y}=109 \mathrm{~m}$. Here stations 50 and 10 are defined as the up- and down-stream ends of the straight section for the $p$ beam. The beam is kicked vertically upward into a series of current-septum magnets. A $1-\mathrm{cm}$ displacement is adequate to clear tine septum. The kicker could be located at. either $\mathrm{C50}$ or A50. The required strength is about $3 \mathrm{kG}-\mathrm{m}$. The present kicker magnet of the Tevatron beam abort system has a rise time of $1.7 \mu \mathrm{sec}$, a length of $2 \mathrm{~m}$ and a peak field of approximately $3.2 \mathrm{kG}$. This design could be adapted for this application.

At $D 50$, the $p$ beam enters a series of ramped current-septum magnets, ( $X$ in Fig. III-4) which further deflect the beam upward by $8.5 \mathrm{mrad}$. Near the end of the straight section at E10, the beam is deflected downward $8.5 \mathrm{mrad}$ to travel at level height and radially outward 12.2 mrad to clear the Tevatron. These deflections are produced by a tilted superconducting dipole ( $T$ in Fig. III-4) similar to the DC dipole but longer by $40 \%$. The septa may be ramped by a half $60-\mathrm{Hz}$ sine wave. The septum can be of $\mathrm{Cu}, 3 \mathrm{~mm}$ thick. For an aperture gap of $1 \mathrm{~cm}$, the peak current is about $10 \mathrm{kA}$. Operating at the duty factor specified here, no cooling is necessary. 
For extraction of the counterclockwise $\vec{p}$ beam, the kicker is located at $B 10$, also in straight section $B 0$. Because of a smaller $\beta_{y}$ of 59 m at $B 10$ and $\mathrm{E} 10$, the necessary kicker strength to kiok the beam vertically downward by $1 \mathrm{~cm}$ at E10 is about 5 to $6 \mathrm{~kg}-\mathrm{m}$. At $\mathrm{E} 10$, the beam enters an extraction channel identical to the $p$ channel, except that it heads downward and clears the Tevatron on the underside instead of on top. The geometry of the extracted beam lines and magnets is shown in Fig. III-4 and the component parameters are given in Table III-3. The cross-sectional geometry of the beans at the entrance to the proton tilted dipole is shown in Fig. III-5.

Table III-3. Parameters of Beam-Transfer Components

\begin{tabular}{|c|c|c|c|c|c|}
\hline & $x_{p}$ & $\mathrm{~T}_{\mathrm{p}}$ & $c_{p}$ & $I_{p}$ & $\mathrm{~K}_{\mathrm{p}}$ \\
\hline $\begin{array}{l}\text { Strength (kGm) } \\
\text { Length (m) } \\
\text { Field (kG) }\end{array}$ & $\begin{array}{l}283 \cdot 5 \\
23 \\
12.33\end{array}$ & $\begin{array}{l}496.0 \\
11 \\
45.09\end{array}$ & $\begin{array}{l}496.0 \\
11 \\
45.09\end{array}$ & $\begin{array}{l}283.5 \\
23 \\
12.33\end{array}$ & $\begin{array}{l}2.67 \\
0.5 \\
5.34\end{array}$ \\
\hline $\begin{array}{l}\text { Deflection (mrad) } \\
\text { Vertical } \\
\text { Horizontal } \\
\text { Total }\end{array}$ & $\begin{array}{l}8.5 \\
0 \\
8.5\end{array}$ & $\begin{array}{l}-8.5 \\
12.2 \\
14.87\end{array}$ & $\begin{array}{l}-8.5 \\
12.2 \\
14.87\end{array}$ & $\begin{array}{l}8.5 \\
0 \\
8.5\end{array}$ & $\begin{array}{l}0.08 \\
0 \\
0.08\end{array}$ \\
\hline $\begin{array}{l}\text { Tilt angle (mrad) } \\
\text { (field from } \\
\text { horizontal) }\end{array}$ & 0 & -2.179 & 2.179 & 0 & 0 \\
\hline \multicolumn{6}{|c|}{$\begin{array}{l}\text { Position of bend-center (m) } \\
\text { Longitudinal }\end{array}$} \\
\hline $\begin{array}{l}\quad \text { from EO } \\
\text { Vertical } \\
\text { Horizontal }\end{array}$ & $\begin{array}{r}-14 \\
0 \\
0\end{array}$ & $\begin{array}{l}20 \\
0.289 \\
0\end{array}$ & $\begin{array}{r}102 \\
0.289 \\
1.000\end{array}$ & $\begin{array}{l}136 \\
0 \\
1.000\end{array}$ & $\begin{array}{l}222 \\
0 \\
1.000\end{array}$ \\
\hline
\end{tabular}

\section{Beam transport and injection into DC}

The geometry of the beam entering the $D C$ is just the reverse of that exiting from the Tevatron. For the $\mathrm{p}$ beam, the tilted superconducting dipole $C_{p}$ (Fig. III-4) identical to $T$ deflects the beam vertically downward $8.5 \mathrm{mrad}$ and radially inward $12.2 \mathrm{mrad}$. The beam is finally deflected upward $8.5 \mathrm{mrad}$ by a series of ramped current septa I identical to $X$ to travel parallel to, but $1 \mathrm{~cm}$ above, the closed orbit in the DC. Bne quarter of a vertical oscillation or one cell. downstream, the beam will cross the closed orbit where it is kicked onto the orbit by a vertical kicker ( $\mathrm{K}_{\mathrm{p}}$ in Fig. III-6) with strength $2.7 \mathrm{kG}-\mathrm{m}$. The kicker should have a fise time (fall time) of $0.5 \mu \mathrm{sec}$. There are several warm spaces that could accommodate this kicker. The $\bar{p}$ beam is identical except that it is injected into the $D C$ from below. 


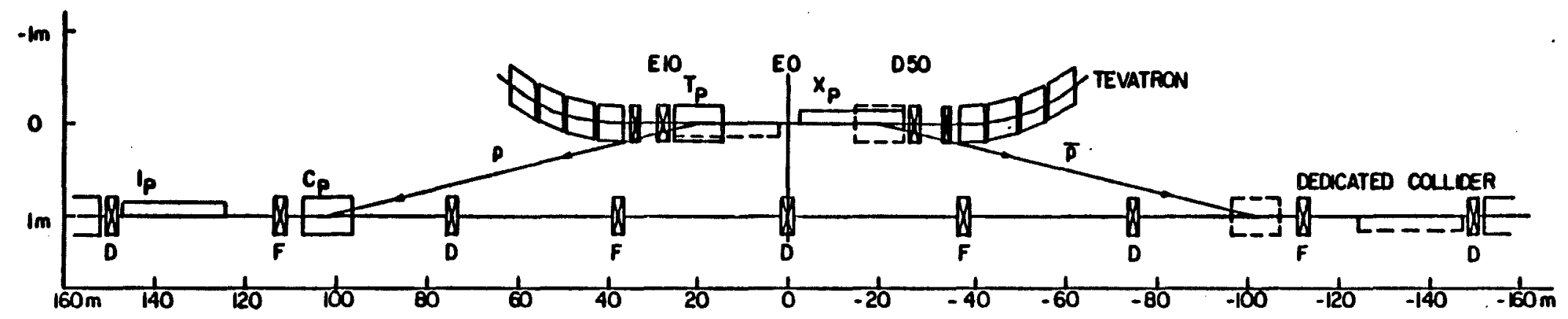

Fig. III-4 Injection from the Tevatron 
In the parameters list of Table III-2, we have neglected for the sake of clarity the vertical displacements of 1 cin caused by the kickers, as well as the vertical separation between the $p$ and the $\bar{p}$ beams caused by the electrostatic separators. These must all be taken into consideration in the detailed design. The vertical orbit geometry at injection is shown more clearly in Fig. III-6. There will necessarily be many bump magnets in the rings for pre-positioning the orbit before kicking the beam. There will also be many steering dipoles all along the beam lines for fine position and angle adjustments.

The matching of optics and dispersion from the Tevatron to the DC has not been done in detail. There appears to be no major obstacle because: 1) matching quadrupoles can be accommodated anywhere in the $70 \mathrm{~m}$ of space between $T_{p}$ and $C_{p}$ and in some spaces outboard of $T_{p}$ and $C_{p}$ and, 2) one can obtaih a fbcal length as short as $15 \mathrm{~m}$ with $p$ quadrupole only $2 \mathrm{~m}$ long.

Beam bunches are injected directly into the DC rf buckets. The $\bar{p}$ bunches are injected first at intervals of one betatron wave length or 4 normal cells apart, namely $300 \mathrm{~m}$ or $1 \mu \mathrm{sec}$. The $\mathrm{p}$ bunches are then injected such that when they arrive at the injection kicker $k$ they are exactly halfway between two neighboring $\bar{p}$ bunches. The rise and fall times of the kickers must therefore be less than $0.5 \mu$ sec so that kicking the $p$ bunches does not affect the $\bar{p}$ bunches.

\section{Beam extraction from DC}

It may be frequently desired to extract a single beam bunch, e.g. an old bunch that has lived beyond its useful life time and is to be replaced by a fresh bunch. To do this, one first decelerates the beans down to $1 \mathrm{TeV}$. Then to extract a $\mathrm{p}$ bunch, one kicks it upward into a series of ramped current-septum magnets $\left(E_{p}\right.$ in Fig. III-6) that are identical to the injection septum $I_{\text {, }}$, but ${ }^{p}$ reversed, which will deflect the $p$ bunch upward $8.5 \mathrm{mrad}$ out of the DC. If the polarity can be reversed, the $\bar{p}$ injection kicker $K_{-}$can be used for the kick. This arrangement is shown in Fig. III-6. Similarly, to extract a $\bar{p}$ bunch, the $p$ injection kicker $k$ with polarity reversed may be used to kick the $\bar{p}$ bunch downward into the extraction septum $E_{p}$. The bunch site vacated by extraction of the $p$ bunch at $E_{p}$ can be refilled ininediately when it arrives at the proton injection septa $I_{p}$ at the end of the long straight section. In this manner, one can replace any beam bunch during a single pass through the injection straignt section.

\section{Beam-abort system}

This system is needed to dispose of the beams rapidly and safely in case of an equipment failure or at the end of a run or a store when the beams are no longer wanted. The predominant consideration in the design 


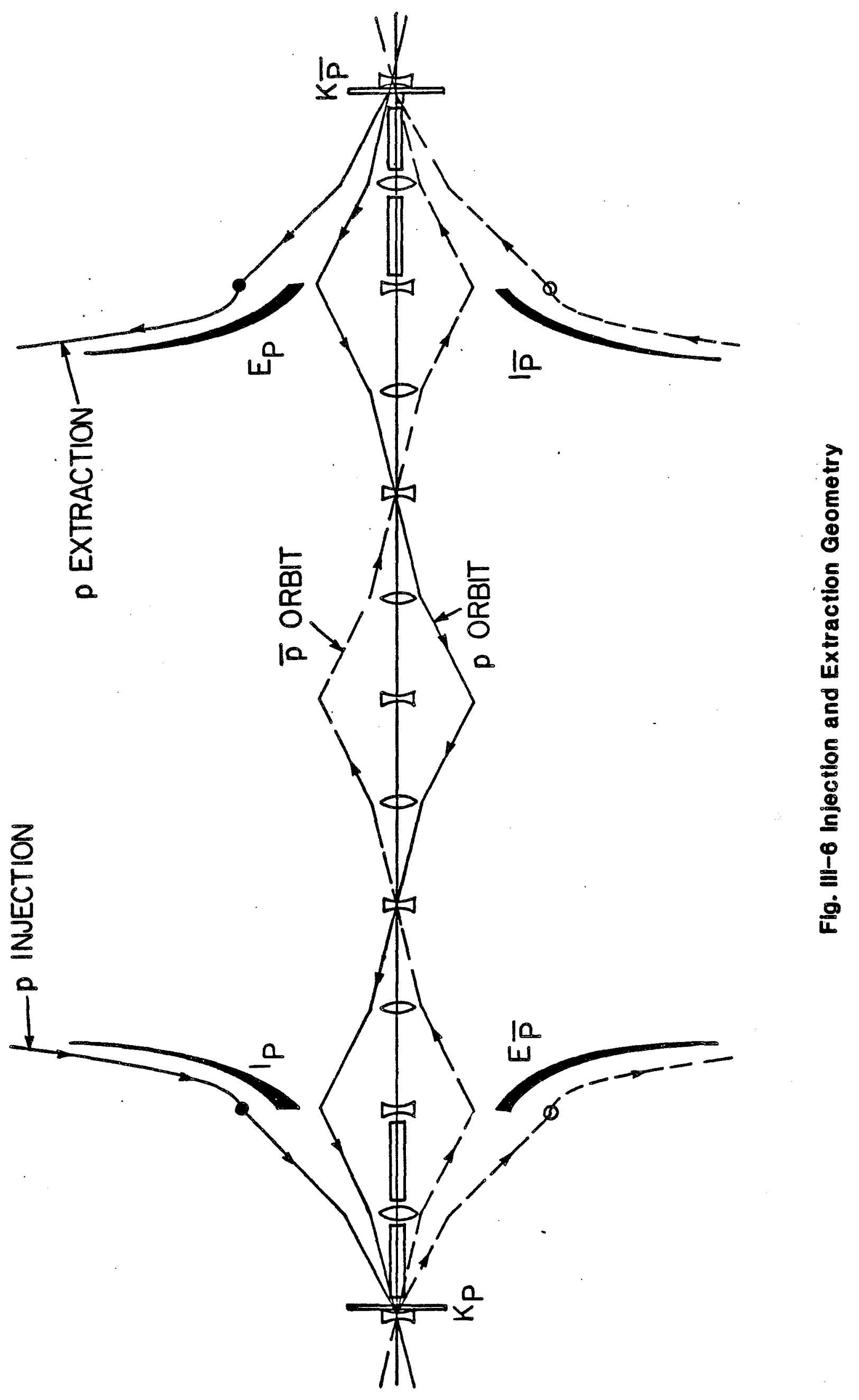


of the abort system is that it should be fast, simple, and reliable. It is a vertical fast beam-extraction system located in the long straight section diametrically opposite to the injection straight section. Because of the large aperture of the Tevatron quadrupoles, a scheme can be devised that is simpler than the standard fast extraction. The $p$ beam is kicked vertically downward at the central defocusing quadrupole $D_{0}$ (Fig. III-7). The kick is the strongest possible that still allows the beam to go through the aperture of the downstream focusing quadrupole $F_{1}$. The beam is further deflected by $F_{1}$ and arrives at the next quadrupole $D_{1}$ at a large enough displacement to pass below on the outside.

A strong kick is needed. The required strength is $68 \mathrm{kG}-\mathrm{m}$ to deflect the 2-TeV beam by $1.02-\mathrm{mrad}$, which gives downward displacements of $38 \mathrm{~mm}=1.5 \mathrm{in}$. at $F_{1}$ and $130 \mathrm{~mm}=5.1$ in. at $D_{1}$. If the present Tevatron abort kicker ( $B l \equiv 10 \mathrm{kG}-\mathrm{m}$ ), is used, we will need 8 sections. These should be placed up- and down-stream of DO with 4 sections on either side. The Tevatron quadrupole has a coil inner radius of 1.75 in., which is sufficient to accomodate a $1 / 16$-in. thick vacuum pipe wall and still allow the beam displaced by $1.5 \mathrm{in}$. at $F$, to go through. The half height of the quadrupole is 5 in., which leaves no tolerance for the beam to pass outside at 5.1 in. One could shave off $1 / 4$ in. from the bottom of the yoke of quadrupole $D_{1}$. One could even build special larger-aperture quadrupoles for $F_{1}$. With a coil inner radius of 2 in., we can deflect the beam by $1.2 \mathrm{mrad}$ using a kicker strength of $73 \mathrm{kG}-\mathrm{m}$. This will give downward displacements of $41 \mathrm{~mm}=1.6 \mathrm{in}$. at $\mathrm{F}$, and $140 \mathrm{~mm}=5.5 \mathrm{in}$. at $D_{1}$. These values give ample tolerance for clearing both quadrupoles. After passing outside $D_{1}$, the beam then continues onto a dump target and is stopped in the target. The target should be made of a low-Z core for the beam to strike, surrounded by a high-Z shield.

Normally, the $p$ and the $\bar{p}$ bunches cross each other at $D_{0}$. Pulsing of the kicker should start immediately after the bunches have passed and peak field should be reached just before the arrival of the next bunches 1 usec later. Both beams will be aborted at the same time. After having received a kick, the $\overrightarrow{\mathrm{p}}$ beam will go through the aperture of focusing quadrupole $F_{0}$, pass outside the defousing quadrupole $D_{-1}$ and be stopped by its own dump target. With $4 \times 10^{12}$ particles on each Earget per abort, there is no danger of damaging the target and the radiation and radioactivity are both minimal.

If at the time when abort is necessary, the beam bunches are for some reason not crossing at DO they should first be brought to crossing at $D O$ by phasing the rf systems. There will be occasions when time will not allow this operation. In this and other cases when the kickers must be triggered at random the worst that can happen is that one bunch of each beam will strike a downstream magnet. This will likely cause the magnet to quench, but should not result in any permanent damage. Such drastic emergencies should be rare. 


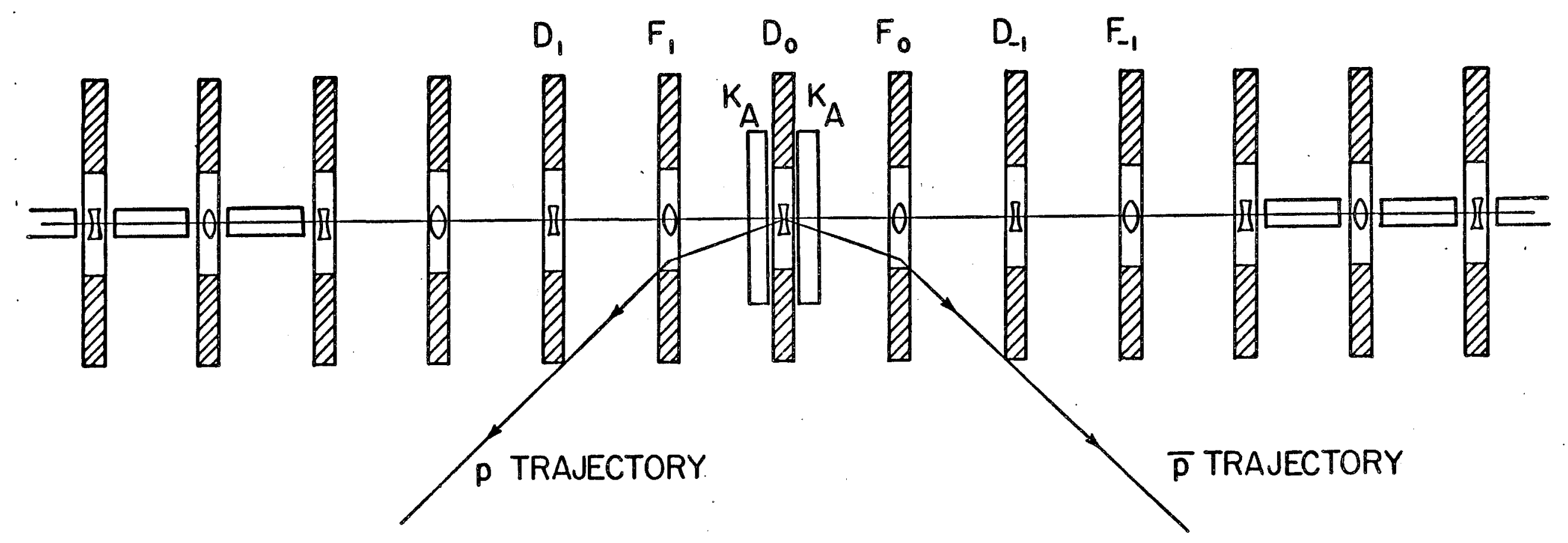

(KA ABORT KICKER)

Fig. III-7 Beam-Abort System 


\section{E. Radio Frequency System}

The radio frequency systems for bunching, accelerating and holding the $\mathrm{p}$ and the $\overline{\mathrm{p}}$ beams are designed to be orthogonal to each other. This is accomplished by placing $\mathrm{rf}$ cavities in pairs spatially separated by a quarter wavelength $(\lambda / 4)$ (or any odd multiple of $\lambda / 4$ ) and operated at a phase difference of $90^{\circ}$. Then, depending on the direction of traversal, the beam bunch will arrive at the cavities sensing an rf phase difference of either $0^{\circ}$ or $180^{\circ}$. In the former case the acoeicraitions will add and in the latter case the accelerations will cancel. In this manner we can have two totally independent $r f$ systems affecting separately and solely the $p$ and the $\bar{p}$ beams.

Since we want the frequency to be the same as that of the Tevatron, we use harmonic number $h=2332$ and we shall see below that $a$ frequency-modulation range of $\Delta f / f=10^{-6}$ is ample. For such a small range, the cavities do not need to be tuned, even though they will have a high $Q$ value, approximately $10^{4}$. A peak voltage per turn of $1 \mathrm{MV}$ for each system is adequate. The $\mathrm{rf}$ and beam bunch parameters at injection (stationary at $1 \mathrm{TeV} / \mathrm{c}$ ) are

Table III-4. RF parameters at injection

\author{
Harmonic number $h$ \\ Frequency $f$ \\ Frequency modulation range $\Delta \mathrm{f} / \mathrm{f}$ \\ Peak voltage per turn $V$ \\ Bucket width $\Delta p / p$ \\ Bucket area $\varepsilon_{0}$ \\ Phase oscillation frequency $\tilde{r}_{s}$ \\ Longi tudinal emittance $\varepsilon_{\ell}$ \\ Bunch length $\delta l( \pm 3 \sigma)$ \\ Bunch width $\delta \mathrm{p} / \mathrm{p}( \pm 3 \sigma)$
}

$$
\begin{gathered}
2332 \\
53.105 \mathrm{MHz}^{\mathrm{MHz}} \\
10^{-1} \\
1 \mathrm{MV} \\
\pm 6.2 \times 10^{-4} \\
14.8 \mathrm{eV}-\mathrm{sec} \\
11.8 \mathrm{~Hz} \\
3 \mathrm{eV}-\mathrm{sec} \\
1.9 \mathrm{~m} \\
\pm 3 \times 10^{-4}
\end{gathered}
$$

The beam bunches are synchronously injected into stationary rf buckets. The bucket area of $14.8 \mathrm{eV}-\mathrm{sec}$ is more than ample to contain the longitudinal emittance $\varepsilon_{l}=3 \mathrm{eV}-\mathrm{sec}$ of the beam bunch.

For acceleration, we will switch to a synchronous phase of $\phi_{g}=15^{\circ}$. This gives an acceleration rate of $0.26 \mathrm{MeV}$ per turn or $5.9 \mathrm{GeV} / \mathrm{sec}$. It will then take 2.8 min to accelerate the beams from $1 \mathrm{TeV}$ to $2 \mathrm{TeV}$. Frequency modulation is unnecessary during acceleration. The velocity change is only $\Delta v / v=0.33 \times 10^{-6}$ and can be compensated by only a very small change in orbit radius. The frequency modulation is to be used mostly for positioning the collision points and to compensate for errors and fluctuations in the magnetic field. The $r f$ and beam paraneters during acceleration are given in Table III-5. 
$\operatorname{III}=14$

Table III-5. RF parameters during acceleration

$\begin{array}{lcc}\text { Bucket area } \varepsilon_{0} & 8.7 \mathrm{eV}-\mathrm{sec} & 14.2 \mathrm{eV}-\mathrm{sec} \\ \text { Bucket length } \Delta l & \pm 3.8 \mathrm{~m} & \pm 3.8 \mathrm{~m} \\ \text { Bucket width } \Delta \mathrm{p} / \mathrm{p} & \pm 4.9 \times 10^{-4} & \pm 3.5 \times 10^{-4} \\ \text { Bunch length } \delta l( \pm 3 \sigma) & 1.9 \mathrm{~m} & 1.6 \mathrm{~m} \\ \text { Bunch width } \delta \mathrm{p} / \mathrm{p}( \pm 3 \sigma) & \pm 3.1 \times 10^{-4} & \pm 1.8 \times 10^{-4} \\ \text { Phase oscillation frequency fo } & 11.6 \mathrm{~Hz} & 8.2 \mathrm{~Hz}\end{array}$

At the top momentum of $2 \mathrm{TeV} / \mathrm{c}$, the synchronous phase is again reduced to zero and the stationary $r f$ and beam-bunch parameters become:

Table III-6. RF parameters at peak momentum

$\begin{array}{lc}\text { Bucket area } \varepsilon_{\mathrm{g}} & 21 \mathrm{eV}-\mathrm{sec} \\ \text { Bucket width } \delta \mathrm{p} / \mathrm{p} & \pm 4 \times 10^{-4} \\ \text { Bunch length } \delta l( \pm 3 \sigma) & 1.6 \mathrm{~m} \\ \text { Bunch width } \delta \mathrm{p} / \mathrm{p}( \pm 3 \sigma) & \pm 1.8 \times 10^{-4} \\ \text { Phase oscillation frequency } f_{\mathrm{s}} & 8.3 \mathrm{~Hz}\end{array}$

The requirements are quite similar to those of the TeV I system, and the Tevatron rf system design could be applied directly to the DC without modification.

The parameters of the Tevatron $\mathbf{r f}$ cavity are

$\begin{array}{lc}\text { Maximum peak voltage } & 360 \mathrm{kV} \\ \mathrm{Q} & 6500 \\ \mathrm{Z}_{0} & 70 \Omega \\ \mathrm{R}_{\mathrm{S}} & 1 \mathrm{M} \Omega\end{array}$

We can use 4 cavities operating at $250 \mathrm{kV}$ peak for each $\mathrm{rf}$ system. The total cavity power per rf system is

$$
4 \times \frac{v^{2}}{2 R_{s}}=125 \mathrm{~kW}
$$

which, although not excessive, is much larger than the beam loading during acceleration. To accelerate 44 bunches of $10^{11}$ protons each at the rate specified above, the beam loading is only $4.2 \mathrm{~kW}$. One may want to reduce the total cavity loss by increasing the number of cavities. In any case, neither the cost of the rf systems nor the cost of the 
cavity is $2.75 \mathrm{~m}$. With the special close-packed geometry proposed for the Tevatron the total of 8 cavities for the $p$ and the $\bar{p}$ rf systems can be accommodated in a free drift length of only $24 \mathrm{~m}$. This space is easily available in a variety of places. We choose a dispersion-matching straight section adjacent to the southwest low- $B$ straight section.

In the DC, with harmonic number 2332, 44 equally spaced bunches of protons or antiprotons will occupy buckets spaced by $53 \mathrm{rf}$ wavelengths. Since 53 is also one of the prime factors of the Tevatron harmonic number 1113, it would be possible, if it appeared desirable, to prepare 21 proton bunches at a time in the Tevatron with correct spacing for injection into the DC.

In the event that a single bunch of antiprotons is to be injected, there are 44 "acceptable locations" for such a bunch in the DC. The single bunch is prepared in the Tevatron at $1 \mathrm{TeV}$ and the rf phases of the two rings slipped slightly until the single bunch is opposite one of the "acceptable locations" in the DC. Then the two rf phases are locked together. In this situation, with the two machines cogging synchronously, the occupied bucket in the Tevatron will advance 1113 buckets in the DC in one turn. This is $2332 / 2$ - 53 buckets. In two Tevatron turns, the advance in the DC will be exactly two "acceptable location" spacings. Consequently, in 43 Tevatron turns, ( $898.7 \mu \mathrm{sec})$, the bunch in the Tevatron will pass opposite each of the "acceptable location" buckets in the DC, and so transfer into any one of the selected buckets can be accomplished with a full turn available to trigger the Tevatron extraction system.

\section{F. Performance of the DC ring \\ 1. Single-beam instabilities.}

The conditions for beam stability are most stringent at injection when the energy is lowest. With a peak voltage of $1 \mathrm{MV}$ and beam longitudinal emittance of $3 \mathrm{eV}-\mathrm{sec}$, the full bunch length at injection is $\delta l=-1.9 \mathrm{~m}$ and the momentum spread is $\delta \mathrm{p} / \mathrm{p} \equiv 3 \times 10^{-4}$ (FWHM). These bunch dimensions do not vary signifigantly during acceleration as long as the synchronous phase is only $15^{\circ}$. At $10^{11} \mathrm{p}$ or $\bar{p}$ per bunch the peak current in the bunch is $I \equiv 3.5 \mathrm{~A}$.

The condition for longitudinal stability is

$$
\frac{\left|z_{\ell}\right|}{n}<F_{\ell} \frac{E / e}{I}|n|\left(\frac{\delta p}{p}\right)^{2} s 19 \Omega
$$


where $F_{f}$ is a form factor of order unity and $E=1$ TeV. Also $n=1 / Y_{t}^{2}-$ $1 / \gamma^{2}=0.60072$ and $\left|z_{\ell}\right| / n$ is the longitudinal impedance of the beam pipe weighted by the spectrum of the particular mode of instability in question. The DC longitudinal stability condition is less stringent than that for the TeV I collider.

The condition for transverse stability is

$$
\left|\mathrm{Z}_{t}\right|<4 \mathrm{~F}_{t} \frac{E / \mathrm{e}}{\mathrm{I}} \frac{\mathrm{V}}{\mathrm{R}}(\delta \mathrm{V}) \sim 2.4 \times 10^{8} \Omega / \mathrm{m}
$$

where $F$ is again a form factor of order unity, $v=44, R=2095$, and $\delta v$ is the intrinsic tune spread in the beam bunch and is taken to be 0.01 . This condition on the transverse impedance $z_{t}$ weighted by the mode spectrum, when translated to that on the longitudinal impedance, gives

$$
\frac{\left|z_{l}\right|}{n}=\frac{b^{2}}{2 R}\left|z_{t}\right| \leq 380 \Omega
$$

where $b=0.038 \mathrm{~m}=1.5 \mathrm{in}$. is the radius of the beam pipe. This condition is of course very much overshadowed by the condition for longitudinal stability.

\section{Luminosity}

For the head-on collision of two beam bunches the integrated luminosity is given by

$$
L=\frac{3 \gamma N^{2}}{2 \beta^{*} \varepsilon_{n}}=4.2 \times 10^{25} \mathrm{~cm}^{-2} \text {, }
$$

where we have substituted

$$
\begin{aligned}
& \gamma=2132(2 \mathrm{TeV}) \\
& \mathrm{N}_{*}=\text { number in each bunch } \quad=10^{11} \\
& \beta^{*}=\beta \text {-function at collision point }=1 \mathrm{~m} \\
& \varepsilon_{n}=\text { normalized transverse emittance }=24 \pi \mathrm{mm}-\mathrm{mrad}
\end{aligned}
$$

This is a rather high luminosity, too high if the detector cannot resolve multiple events. For such a detector $i$ must be $<10^{25} \mathrm{~cm}^{-2}$. Nevertheless if we accept this value and with a revolution frequency of $23 \mathrm{kHz}$ and 44 bunch-collisions per revolution, we find 


$$
\mathcal{L}=4.2 \times 10^{31} \mathrm{~cm}^{-2} \mathrm{sec}^{-1}
$$

\section{Beam-beam limits and Luminosity life time}

Although not much is known about the detailed effects of the beam-beam interactions, the effect is generally believed to be measured by the linear tune shift $\Delta \nu$ and the limit of $\Delta v$ is taken to be approximately 0.005 . Straightforward calculation gives

$$
\Delta \nu=\frac{3}{2} \frac{r_{p^{N}}}{\varepsilon_{n}}=0.003 / \text { crossing, }
$$

which is acceptable. A factor 10 increase would not be acceptable according to the lore. But until it is actually demonstrated to be impossible, it should not be written off. The total tune shift per revolution with 4 crossings is $4 \Delta v=0.012$ and is also acceptable.

The luminosity lifetime due to scattering by residual gas has been estimated for the Tevatron collider to be 110 hours. This contribution is (assuming the same vacuum for Tevatron and DC) independent of energy and causes no problem not met already in the Tevatron collider.

Likewise, the beam lifetime in the Tevatron due to intrabeam scattering has been calculated. For parameters corresponding to those used here for 1-TeV injection into the DC, the lifetine $90 r_{1}$ particles. This result can be scaled to the 2-TeV beam in the DC. The result is a slight decrease in lifetime, to something around 40 hours. The emittance growth is mainly in longitudinal phase space. Performance degradation from this bunch lengthening therefore depends on intangibles such as the amount of $\mathrm{RF}$ noise present. The lifetime for horizontal emittance growth is approximately 100 hours, while the vertical emittance (in the absence of coupling) does not grow at all.

The beams are depleted by nuclear interactions. Assuming (conservatively) a $\mathrm{p} \overline{\mathrm{p}}$ total cross-section of $100 \mathrm{mb}$ and assuming (conservatively) certain extinction of a beam proton if it interacts with a $\bar{p}$, one obtains an attrition lifetime from beam-beam collisions themselvess of about $73 \mathrm{hr}$ at a total Iuninosity of $4 \times 4.2 \times 10^{31} \mathrm{~cm}^{-2} \mathrm{sec}^{-1}$. This is not a bad match to the 44 hours required to accumulate 44 bunches of $\bar{p}$ at $10^{11}$ per bunch.

Calculations of luminosity versus time have been carried out including all these effects, as well as effects of emittance growth due to multiple scattering. The result is shown in Fig. III-8. The initial luminosity lifetime is $15 \mathrm{hrs}$, but the time dependence is not 


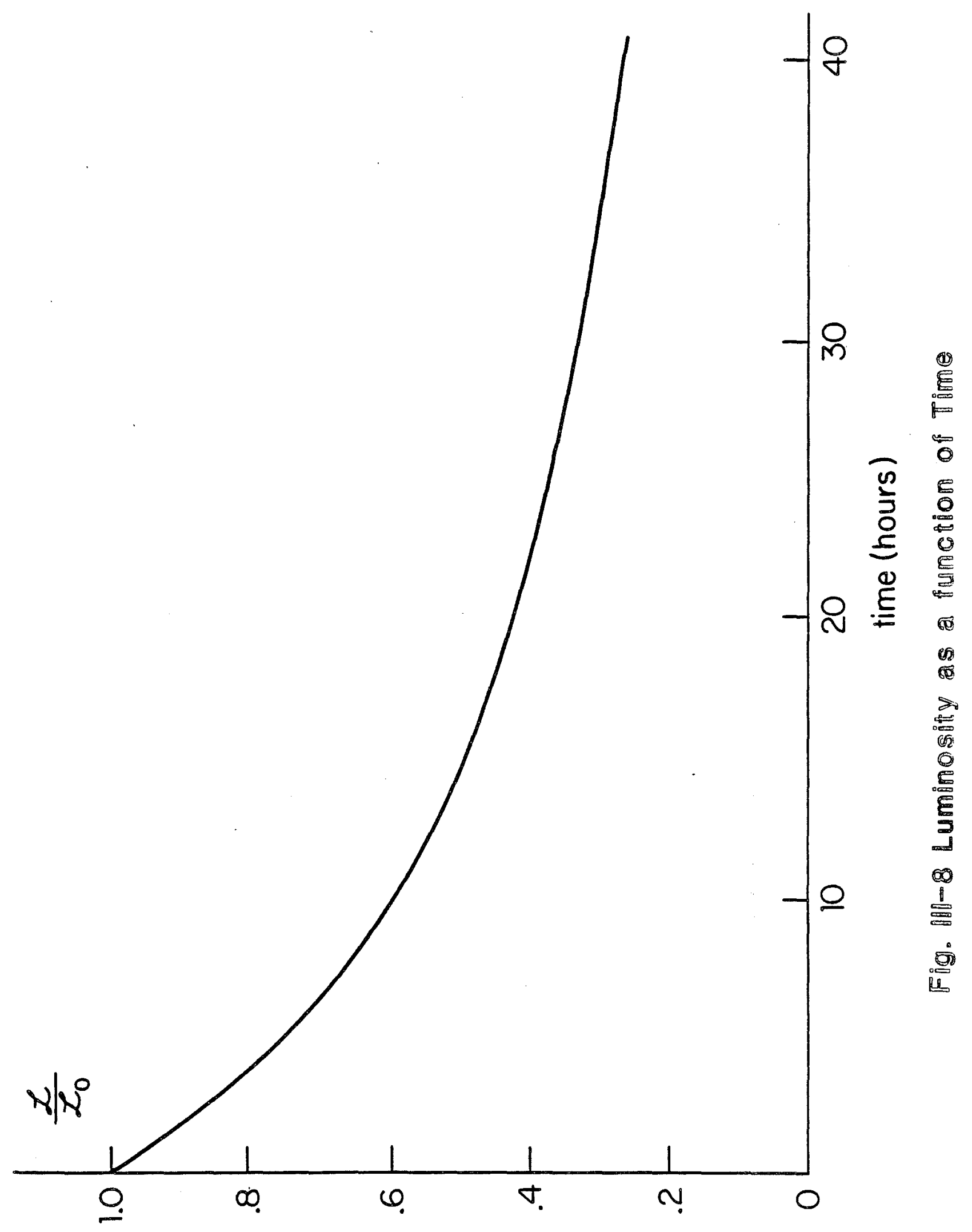


exponential. An average luminosity of over $2 \times 10^{31} \mathrm{~cm}^{-2} \mathrm{sec}^{-1}$ looks quite at tainable.

The factors that in practice limit the iuminosity lifetime may well be quite different and more complex, involving interplay between the beam-beam force, lattice nonlinearities and noise. The best design guidance will come from working experience with the SPS collider (the results of which are already encouraging) and with the Tevatron collider.

\section{G. Operating scenarios}

Many factors, including $\bar{p}$ production rate, naximum charge per beam bunch, beam-transfer time, saturation time of the $\bar{p}$ accumulator core, and beam lifetime in the DC will determine the optimum strategy for operating the $D C$ in conjunction with other possible operations of the Tevatron. Several basic time scales are apparent, however. The $\bar{p}$ source should continue to use single Booster batches from the Main Ring at a rate of $1 / 2 \mathrm{~Hz}$. Roughly once a minute, $\bar{p}$ production could be interrupted to fill the Tevatron for a fixed-target pulse. $\bar{p}$ production could resume with a loss of only one or two pulses for a duty factor of more than 90\%. At the rate of $10^{11}$ per hour, after several hours the $\bar{p}$ 's accumulated in the core of the Accumulator will be transferred to the Main Ring, Tevatron and DC. A full set of protons will be injected at this time and the $D C$ will then be ramped to $2 \mathrm{TeV}$ for the next colliding beam run of several hours.

The most critical parameters determining the average luminosity are likely to be the average $\bar{p}$ production rate and single-beam lifetime in the DC. Conservative estimates based on bile $\mathrm{TeV} I$ design give a projected $\bar{p}$ production rate of $10^{11} \bar{p}$ /hour, or one DC bunch per hour. Thus, a given bunch out of the 44 stored in the DC can be replaced once every 44 hours on average. If the beam lifetime $T_{p}$ is much less than this value, the average number of antiprotons and hence the average luminosity is a factor of $T_{p} / 44$ hours lower than the optimum value. In this lifetime regime, it might be advantageous to run with fewer bunches if the corresponding operating efficiency could be improved. The number of buncines could be reduced to approximately $T_{p}$ (hours). (This rule applies because one $\bar{p}$ bunch is produced in Bne hour). Clearly it is important to maximize the antiproton production rate and the beam lifetime, although the estimates of both of these quantities (discussed above) appear satisfactory.

The time between refills of the DC, will probably be set by the time for the $\bar{p}$ core in the accumulator to saturate. Inis is expected to be in the range 10-20 hours. Assuming the DC single beam lifetime is much greater than this value, one would wait for the $\bar{p}$ accumulator to saturate, then refill the 10 to 20 oldest $\bar{p}$ bunches in a single cycle of ramping the DC down to $1 \mathrm{TeV}$. The entire fill of protons could be replenished at this time. 
For injection or refill, the ring magnets of the DC are brought to the level of $1 \mathrm{TeV}$. The low $\beta$ insertion quadrupoles and the electrostatic beam orbit separators should.all be turned on and tuned so that the $p$ and $\bar{p}$ orbits are properly separated. The rf system should be fully turned on and properly tuned. The $\bar{p}$ bunches are injected first. They are provided from the Tevatron at $1 \mathrm{TeV}$. The Tevatron $\mathrm{rf}$ should be locked to the DC rf before injection so that the injected beam bunches are synchronously captured into the DC rf buckets.

It is likely that 21 bunches of protons can be accelerated to $1 \mathrm{TeV}$ in one Tevatron pulse. The Tevatron field is then flat-topped and the Tevatron $\mathrm{rf}$ is locked to the DC rf. The proton bunches are then transferred to the $D C$ at a rate limited only by the power supplies of the extraction and injection kickers. The injection of proton bunches should take no more than a few minutes. Both are then accelerated to $2 \mathrm{TeV}$. Throughout the injection and acceleration processes, the frequencies of the $p$ and $\bar{p}$ rf systems are fixed and locked to each other. At $2 \mathrm{TeV}$, the frequencies are carefully trimmed to position the beam collision points at the desired locations in the detectors. This process of acceleration of the beams and bringing them to proper collision should take only a few minutes.

Thus, the optimum running scenario is expected to be as follows: 1. ) The Main Ring provides a Booster batch to the $\bar{p}$ source every 2 seconds. 2.) Every minute, $\bar{p}$ production is halted and 13 Booster batches are accelerated by the Main Ring and to the Tevatron for a rixed-target pulse. 3.) Every 10 to 20 hours, fixed-target operation is halted, and the $D C$ is ramped down to $1 \mathrm{TeV}$. The stored protons are extracted and 10 to 20 new bunches of antiprotons are injected from the Accumulator chrough the Main Ring and the Tevatron. A new fill of proton bunches is injected from the Tevatron and finally the DC is ramped back to $2 \mathrm{TeV}$ and colliding-beam operation resumes. This entire cycling operation should require iess than 10 minutes.

\section{H. Bunch-Avoidance Beam Dynamics}

As the proton and antiproton bunches pass each other in the normal DC lattice, they encounter attractive impulsive forces. This leads to various effects on perforrance:

1. The wavelength of the sinuous design orbit decreases in the presence of the counterrotating beam. For the design parameters ( 44 buncines $10^{11}$ /bunch) corresponding to full luminosity of $4 \times 10^{31} \mathrm{~cm}^{-2} \mathrm{sec}^{-1}$, the estimated wavelength change is $0.3 \%$. Tuning of the voltages on the electrostatic separators $S, S^{\prime}$, and S'' provides sufficient compensation for this effect.

2. The vertical tune decreases in the presence of the counterrotating beam. This effect is distinct, from the 
wavelength decrease described above. The estimated total linear tune shift from these close encounters is no more than .012. It is of opposite sign to the linear tune shift (.012 total) produced by the direct beam-beam collisions and, at least from that point of view, is not unwelcome. In practice, these close-encounter tune shicts of $p$ and $\bar{p}$ will depend (as will the direct beam-beam tune shift), upon the absolute and relative intensities of $p$ and $\bar{p}$ beams and will vary from run to run and even during a run. Therefore these tune shifts will need to be compensated by a correction system which in particular can control tune differences of $p$ and $\bar{p}$ bunches (especially in the vertical plane).

To correct the vertical tune difference of $p$ and $\bar{p}$, electrostatic quadrupoles of reasonable gradients $\left(10 \mathrm{kV} / \mathrm{cm}^{2}\right)$ and length $(1 \mathrm{~m})$ located adjacent to the electrostatic deflection plates $S$ suffice to provide adjustment of the vertical tune difference of $p$ and $\bar{p}$ beams over a range $0 \leq\left|\Delta \nu_{v}\right| \leq .08$. Similar systems located at high $\beta_{H}$ (and low $\beta_{v}$ ) can correct, if necessary, horizontal tune differences and/or chromaticity differences of the $p$ and $\bar{p}$ beams.

3. Given that these linear tune shifts are compensated with correction elements, there still remains a tune spread originating from the close encounters. The tune spread is a rough measure of the importance of nonlinear beam-beam interaction effects associated with these forces. We find that, because of the large bunch separations, the tune spread is small $(<.002)$ in comparison to the contribution of all the direct beam-beam collisions (.003/crossing; 4 crossings).

4. Rigid betatron motion of the $\bar{p}$ bunches modulates the force on the $p$ bunches (and vice versa), leading (in good approximation) to a linear coupling of the betatron motions of the counterrotating beams. This is a tractable problem already studied, among others, by Chao and Keil (cf. E. Keil, 1981 CERN Accelerator Concerence, p. 759), albeit in a somewhat simplified form. They find stable inoision for all modes provided that the total linear tune shift from the bean-beam encounters is small compared to 0.1 , and that the tune is not chosen near an integer. These criteria are clearly satisfied by the DC.

Studies generalizing those of Chao and Keil to DC conditions of 88 bunches, including unequal bunch populations and direct as well as close-encounter linear beam-beam effects, have been carried out. They demonstrate that a sufficient condition for linear stability of all modes is that the tune difference of inperturbed $p$ and $\bar{p}$ beams exceed the beam-beam tune shifts. (of course, integer tunes must also be avoided.) If this condition is also necessary (unlikely but not strictly ruled out), the electrostatic quadrupoles sufice to provide the requisite tune split.

The above list does not represent an exhaustive compendium of problems. Remaining ones may be more nonlinear. This will mean that not only are they harder to estimate but also, even when estimated, the 
results will be more controversial. Such problems are best attacked with numerical tracking studies once the final lattice design has been determined. However, just as the conventional beam-beam linear tuneshift provides a convenient - if superficial - measure of the severity. of nonlinear beam-beam effects, we believe that the above estimates provide reasonable evidence that the nonlinear effects of the multibunch close encounters will not be severe. We also note that feedback systems are available to damp any residual coupledwounch instabilities. A bandwidth of $1-10 \mathrm{MHz}$ is sufficient. 


\section{PROTON RING COMPONENTS}

\section{A. Magnets}

The magnets to be used in the DC are very similar to the Energy Saver magnets. They have been described many times in great detail; only modifications to the Saver design will be discussed here.

1. Peak field: The required field for 2-TeV operation of the DC is $4.65 \mathrm{~T}$, slightly larger than the design specification for the Saver. Actually, this field has been achieved by most of the last dipoles built. The increase comes from improved quality of the superconducting wire. Adoption of the cable used for low- $\beta$ quads would require no design changes and would provide fields considerably in excess of the design goal of $4.65 \mathrm{~T}$. See also Chapter IX.

2. Length of dipoles. The length of the DC dipoles, $7.75 \mathrm{~m}$, is significantly greater than the 6.1-m Saver dipole, but this increase has little impact on fabrication technique or performance. The requirements for the quench-protection system are somewhat different, but will not require any basic design modification.

3. Cryogen flow through the dipole: Because the length of a magnet string served by a single satellite refrigerator is doubled, the temperature difference across a given dipole is halved. In the DC, very little heat is generated by ramping losses because the ramp will be so slow. A small decrease in cryostat impedance, to be realized by small changes in internal flow paths, will achieve this reduced temperature difference. Cooldown will be at most twice as slow.

4. Quadrupole parameters. The standard quadrupole for the DC is lengthened $2.5 \mathrm{~m}$ from the $1.7 \mathrm{~m}$ Saver length and the peak gradient is increased to $1.0 \mathrm{~T} / \mathrm{cm}$. This value is obtained in the $\mathrm{TeV} I \mathrm{l}$ low- $\beta$ quads and no significant design changes are required. Quadrupoles longer than this have already been produced.

5. Spool pieces: As in the Saver, the spool pieces will contain trim dipole and quadrupole coils as well as sextupole and octupole windings. As described in Chapter III, the strength of sextupole and octupole corrections needed for the DC is estimated to be about twice that used in the Saver, while the trim-dipole strength is the same and the quadrupole strength is 4 times that of the Saver. These increases can be accommodated by lengthening the spool package and operating the trim system at higher currents.

6. Low- $\beta$ quadrupoles: The specifications for low- $\beta$ quadrupoles given in Chapter III do not exceed the design for the TeV I collision region. No special problems are expected. 


\section{Other special magnets.}

a. Kickers: Kickers are required for Saver extraction, DC injection, and DC extraction. The parameters given in Chapter III are similar to those used for the Saver. The kickers used for the abort system again are similar to those used in the Saver, although a sequence of $4+4$ such kickers is required.

b. Septa: Ramped current septa, ( $60 \mathrm{~Hz}$ half sine wave) to be used for Saver extraction and DC injection have been used in the Booster extraction system. A total of 450 linear feet of these devices will be required. The septa can be made of 3-mm thick copper. For a $1-\mathrm{cm}$ aperture gap the peak current is about $10 \mathrm{kA}$. Peak field is $1.2 \mathrm{~T}$.

c. Long extraction dipoles. The 11-m superconducting dipoles used immediately downstream of the extraction septa may be subjected to a heavy heat load by stray radiation. Several solutions to this problem have been suggested. It will be possible to test these solutions with the Energy Saver.

Assembly of the superconducting magnets will be carried out just as for the Saver in the existing Technical support Facilities. Prototyping, retooling, and restart of the magnet-measurement facility will require one year from the starting date, i.e. during FY 85 . Thereafter magnet production will proceed at a nominal rate of 15 dipoles and quads/week. A three-year production schedule is sufficient for supplying the normal dipoles and quadrupoles. Some expansion of existing facilities will be necessary to supply in addition the rather large number of correction elements and special devices within a three-year period.

\section{B. Refrigeration System}

The pertinent parameters for the Dedicated Collider that are needed for the design of the cryogenic refrigeration system are:

1. Diameter to be twice that of the main ring.

2. The center of the ring is at the industrial area.

3. Saver magnets are to be used.

4. The DC is a single pp ring.

5. There are $4 \mathrm{p} \overline{\mathrm{p}}$ interaction regions, with two contiguous ep interaction regions.

This leads to approximately twice the number of superconducting magnets as in the Saver. Since the ramp-up to full field is to be 
approximately 2.8 minutes and since this will only occur infrequently, we assume that the refrigeration allowance for AC loss can be ignored.

The Energy Saver refrigeration requirement is about half static loss and half dynamic, and the proposed refrigeration system for the DC ring therefore consists of duplicating the Energy Saver system now being commissioned. The new system will have a new central helium liquefier and 24 satellite refrigerators distributed around the ring. The central liquefier is connected to the satellites via a transfer line similar to the one installed for the Energy Saver. After studying the relative costs of a distributed compressor system for the satelites, we have elected to provide a central compressor station for the satellites connected by a high pressure supply line and a low-pressure return line. This selection reduces the electrical distribution-system costs and therefore is the lower-cost solution. In order to allow for refrigeration needs for special quads for interaction regions and for superconducting detector magnets, an additional satellite refrigerator has been added to the complex for each interaction region.

The Energy Saver Central Liquefier uses 2000-hp reciprocating compressors that were obtained as surplus property from the US space program. Fermilab funded out of its budget the reconditioning and move of these units. With the new project, it is likely that the BNi study, which led to selection of oil-lubricated screw compressors sirallar to Fermilab's satellite compressors, is applicable. The proposed new CHL therefore incorporates rotary oil-lubricated serew compressors. The cold-box supplied by Helix Technology Inc., (Helix is now Koch Process Systems), could be replicated without redoing the engineering, and substantial cost savings could be possiole. We niave asked Helix to verify the cost of supplying an additional unit lasing 1983 dollars. Since they are the contractor for the BNL compressor systern, they will also supply a cost estimate for an appropriately matched compressor system. This procedure minimizes the impact of DC studies on the Fermilab staff during the commissioning of the Saver and TeV I and II.

Refrigeration for the DC magnets will be supplied by each of the 24 satellites to a string of 64 dipole magnets and 16 quadrupole magnets ( 600 meters total length). Single-phase liquid helium at a pressure of 14 psig is forced through the magnet string from the center feed-point towards both ends, where the helium is expanded through throttling valves. Two-phase helium then returns to the feed point through $a$ concentric tube. Heat transfer oours between the single-phase and two-phase flow at a sufficient rate to maintain approximate constant temperature of the superconducting magnet coils $(\Delta \mathrm{T}=0.2 \mathrm{~K})$. This system is the saine as that in use for the Energy Saver. The important difference is the length is about twice that of the Energy Saver. This requires that the outer 2-phase return tube on the cryostats have a slightly larger diameter in order to keep the 2-phase pressure drop $(\sim \sigma \mathrm{psi})$ the same. This is a minor change in the magnet cryostat.

A new Central Helium Liquefier building of the same design and size will be constructed in the vicinity of the present CHL. In is will house 
an oil-lubricated screw compressor system sized to deliver $1300 \mathrm{~g} / \mathrm{sec}$ of helium gas at $300 \mathrm{~K}$ and $13 \mathrm{~atm}$ as well as purifiers and a cold box. The compressors will require approximately $9000 \mathrm{hp}$. The cold box uses $0.7 \mathrm{l}$ of liquid nitrogen per liter of liquid helium produced. This liquid nitrogen will be provided by the Energy Saver's new nitrogen-reliquefier plant. The capacity for the CHL II will be adequate to provide $100 \mathrm{l} / \mathrm{hr}$ of liquid He to each of the 30 satellite refrigerators. The reserve capacity will be $50 \%$.

The compressors for the 30 satellite refrigerators will be located in a central compressor building in the vicinity of the two central liquefiers. These 350-hp oil-lubricated screw compressors could be of the same make as the Energy Saver (Mycom) or from a different maker if a more favorable price could be obtained. The primary advantage of a single satellite compressor station near the distribution center of the utilities (electric and water) is that it avoids a long electrical feeder around the ring and a similarly distributed cooling-water system. We have calculated the size of the required low-pressure return line and find that the largest pipe size is 20 in. in diameter. The return flow falls of $f$ as one proceeds around the ring and it will be cost effective to reduce the line size, first to 16 in. diameter, then by $2-i n$. steps until the final connection of 8 in. diameter. In addition to this large low-pressure return line, the distribution system will include a high-pressure (20 atm) helium-gas supply line and a liquid-helium transfer line similar to the 6000-m Energy Saver transfer line.

Each satellite refrigerator receives $50 \mathrm{~g} / \mathrm{sec}$ of 20-atm gas from the central satellite compressor station. This gas passes down the heat exchanger column and is cooled to $6 \mathrm{~K}$. It is then expanded by the wet reciprocating expansion engine to $1.8 \mathrm{~atm}$. This cold flow is joined by low-temperature helium from the central liquefier. Aftier merging, the helium flow passes through a sub-cooler and enters the magnet string at 4. $4 \mathrm{~K}$.

The capability of this system can be judged from the results achieved during the commissioning of the Energy Saver, when sectors $D, E$ and $F$ of the Energy Saver were cooled to 4.5K on February 26, 1983. This half-ring cooling operation was initiated at the beginning of 1983 and only minor difficulties have been experienced. For the first half of 1982, "A" sector tests were also conducted without serious refrigeration-system problems. We believe this experience shows the soundness of this liquid-helium temperature refrigeration-system design and therefore justifies using the same concepts for DC studies.

\section{Vacuum System}

The design of the vacuum system is taken directly from the Energy Saver. Details may be found in the Saver Design Report and will not be repeated here. The feature that most directly impacts on DC performance is of course beam lifetime, which was discussed in section $F$ of Chapter III. The estimated single-beam lifetime in the Saver from gas 
scattering (mainly the loss due to highly inelastic collisions) is 110 hrs. This loss comes largely from the higher gas pressure ( $\cong 10^{-8}$ Torr) in the six Saver warm straight sections, which amount to $10 \%$ of the circumference of the ring.

The DC has a larger fraction $(8 / 44 \equiv 18 \%)$ of its circumference in straight sections. Four of these, the utility straight sections, are analogous to the Saver sections. The other four, the low- $\beta$ collision straight sections, have a considerable number of magnets in them, and will have additional pumping in order to minimize backgrounds for the detectors. Thus the fraction of DC circumference that is warm is no more than 15\%, leading to a beam lifetime no less than $70 \%$ of that in the Saver, or 80 hrs.

Other possible limits on performance are also common to the Saver and the DC. These include the pressure-bump and trapped-electron (or ion) instabilities. For $p \bar{p}$ operation, the first should not be a problem because of the very low average beam current. The second is also unlikely to be a problem for $p \bar{p}$ operation, because of the 1-usec separation of the bunches, not to mention the charge neutrality of the time-averaged beam distribution.

Even for ep operation, neither of these problems should arise. Calculations and measurements indicate that the ring could carry at least an additional factor 10 of protons before the pressure-bump instability would become a problem. There must also be a large gap ( 1 usec) in the circulating beam to accommodate the rise of the abort kicker. This gap,together with rf bunch structure, should give sufficient time for electrons to be swept from the beam region.

\section{Controls System \\ 1. General}

In keeping with the general philosophy, the controls system should avoid the need for new design and should capitalize on the developments that have already been successfully installed on the Energy Saver. In any case, treating the new complex in exactly the same way as the existing ones will enable the current consoles to be used for control of any accelerator or storage ring on the site, an operational advantage not to be lightly thrown away.

Considering the DC controls complex to be an extension of the existing facility has the additional advantage that a considerable hardware infrastructure can be shared, especially operational spares and program-development facilities. In addition, much of the necessary software, especially systems software, in which many years of effort has been invested, can be used without change on the new rings. 


\section{Equipment Interface}

Following this philosophy, we will install additional CAMAC loops for the equipment of the new complex and even use MULTIBUS-based distributed-microprocessor systems in much the same way as is done at the Energy Saver.

\section{Computers}

Extra front-end computers will be needed for the DC. Experience so far obtained from the VAX-PDP11 system indicates that the following groups of equipment will need a dedicated front-end computer each:

Electron Linac

Electron Accelerator

Power Supply control for the DC and its low- $\beta$ sections and the beam transport.

All other DC control (cryogenics, rf, vacuum, etc)

In addition, there will be a need for an additional VAX-11 to carry the supplementary data-base and library load. The cost of a second VAX can probably be avoided, because backup and programming support facilities already exist.

The network facilities currently installed at the Main Ring and Energy Saver are adequate to carry the extra load and the additional computers can be housed in the vicinity of those already installed.

There are good reasons for supposing that cheaper, faster versions of both computers will be available in program-compatible form. Use of these would not necessarily be an advantage if it meant carrying more spares, but this decision can be made at the time of purchase.

\section{Consoles}

By the time the Tevatron is established and reliable enough to be used as an injector for the $\mathrm{DC}$, console activity in the established part of the complex will be quiet enough to enable the existing consoles to be used for the DC. On the other hand, additional consoles may well be required for local control of the new electron Linac and Accelerator during commissioning and after shut-down.

\section{Software}

One of the greatest advantages of abstaining from the use of new principles in the building of the control system is that much of the necesary software can be picked up unchanged and in working condition from the work already done on the Main Ring and Energy Saver. 
The operating systems, the ACNET software and the entire data-base structure can be obtained substantially without programming effort. This is obviously true of the console-support software. It is equally true of large areas of local microcomputer systems dealing with cryogenics, vacuum, and power-supply control. How much of this work can be directly transported from the Energy Saver will depend on the degree of determination of various systems groups to use their hardware unchanged.

A comprehensive suite of application programs will be needed for the new rings, but even here programs of the "parameter-page" type may come ready-made, needing only the attribution of suitable parameters. It is clear that the programming effort now being used to commission the Saver and TEV II projects can pass without strain to these new rings, satisfying the deadline requirements for sof tware at about the correct pace. The new software can therefore be built by the existing controls group staff when they have finished building and commissioning the software for the earlier rings.

\section{E. RF System}

As indicated in the previous Chapter, the $\mathrm{rf}$ system can be taken over directly from the Saver. A total of eight cavities is needed. They are conveniently located in a dispersion- matching straight section, with an $\mathrm{rf}$ gallery above containing anode power supplies, modulators, power amplifiers, controls, etc. A location adjacent to the $\mathrm{SW}$ collision hall is chosen. It is close to the Main Ring and Saver rf system and to those power and water systems.

An exact duplication of the Tevatron $r f$ system design would meet the requirement of the DC; thus a proof of principle and a cost basis can be derived therefrom. However, the Tevatron $r f$ cavities were, of necessity, designed to be long and thin so as to fit under the existing Main Ring $r f$ cavities. This restriction does not exist in the $D C$, and $a$ slightly different cavity geometry could be used. By changing the shape slightly, a higher operating efficiency might be achieved. An added benefit would be a significant shortening of the cavities, so that the entire system would occupy a shorter space in the DC lattice. RF cavities of slightly modified geometry would not result in any significant change in the cost of the rf system. 

The existence of a $2-\mathrm{TeV}$ proton ring at Fermilab will present a unique opportunity for the observation of extremely high energy electron-proton collisions with an electron storage ring of modest energy. Here we describe a $10-\mathrm{GeV}$ electron ring tangent to the 2-TeV DC at the utility straight section near the village. The electron ring has a 350-m long straight section that contains two interaction areas with longitudinally polarized electrons available in each. Luminosities of $6 \times 10^{31} \mathrm{~cm}^{-2} \mathrm{sec}^{-1}$ can be reasonably expected. The electron-ring parameters are listed in Table V-1 and the layout of the ring is shown in Fig. $\mathrm{V}-1$.

\section{Table V-1. Electron-Ring Parameters}

$\begin{array}{lll}\text { Energy } & 10.0 & \mathrm{GeV} \\ \text { Injection Energy } & 5.0 & \mathrm{GeV} \\ \text { Circumference } & 1659.7 & \mathrm{~m} \\ \text { Number of Bunches } & 98 & \\ \text { Bunch Separation } & 16.94 & \mathrm{~m} \\ \text { Bunch Frequency } & 17.7 & \mathrm{MHz} \\ \text { Electrons/Bunch } & 8.5 \times 10^{10} & \\ \text { Emittance (Horizontal, rms) } & .035 & \mathrm{~mm}-\mathrm{mrad} \\ \text { Emittance (Vertical, rms) } & .016 & \mathrm{~mm}-\mathrm{mrad} \\ \text { Energy Spread } & 1.2 \times 10^{-3} & \\ \text { Tune (Horizontal/Vertical) } & 37.1 / 36.2 & \\ \text { Momentum Compaction } & 6.7 \times 10^{-4} & \\ \text { Polarization Time } & 14.8 & \mathrm{~min} \\ \text { Equilibrium Polarization } & 80.5 & \% \\ \text { Energy Loss/Turn } & 13.2 & \mathrm{MeV} \\ \text { Damping Time (Transverse) } & 8.4 & \mathrm{msec} \\ \text { Bending Field } & 3.4 & \mathrm{kG} \\ \text { Number of Interaction Regions } & 2 & \\ \text { Beam Size at Interaction Point } & & \\ \text { Beam Size at Interaction Point } & 0.13 & \mathrm{~mm} \\ \quad & 0.09 & \mathrm{~mm}\end{array}$

A. Luminosity

The bunch spacing shown in Table $\mathrm{V}-1$ requires a rebunching by three in the Main Ring, producing one bunch every $56.6 \mathrm{nsec}$ containing $6.9 \times 10^{10}$ protons. This results in 742 proton bunches in the DC with a total of $5.1 \times 10^{13}$ circulating protons. The proton beam is assumed to have an emittance $\varepsilon=0.01 \pi \mathrm{mm}-\mathrm{mrad}$, the same value as in the Tevatron. 




RING DIMENSIONS

F1g. V-1. Physical Dimensions of the $10 \mathrm{GeV}$ Electron Storage Ring (all dimensions are in meters). 
With a $\beta^{*}$ in the DC of $8.5 \mathrm{~m}$, the electron beam can be matched to the proton beam with an emittance of $\varepsilon / \pi=0.025 \mathrm{~mm}-\mathrm{mrad}$ and a $\beta^{*}$ of $0.55 \mathrm{~m}$ in both transverse planes. We have chosen round colliding beams and zero-angle crossing between electrons and protons for several reasons. A round beam is the more natural configuration for the proton beam, and the presence of vertical bending magnets within the electron ring (used for polarization rotation) produces a nearly round electron beam even in the absence of vertical/horizontal coupling. Zero-angle crossing allows for the use of meter-long proton bunches and so does not place any stringent requirements on the proton $\mathrm{RF}$ system.

The luminosity is estimated at $6.2 \times 10^{31} \mathrm{~cm}^{-2} \mathrm{sec}^{-1}$ as shown in Table $\mathrm{V}-2$. The required electron current is $240 \mathrm{~mA}$ and the assumed tune-shift limits are $\Delta \nu_{0}=0.030$ and $\Delta \nu_{0}=0.0040$ (per interaction region). The assumed electron and proton lune shifts are consistent with the present experlence in existing colliding-beam facilities. However, the electron ring described here has fairly good damping and there is a preliminary indication that the use of a round beam might allow one to survive higher tune shifts than in the $e^{+} e^{-}$machines currently operational. Since Table $\mathrm{v}-2$ reflects a total number of protons that is perhaps $33 \%$ below the capabilities of the $D C$, the prospects for raising luminosities toward $9 \times 10^{31} \mathrm{~cm}^{-2} \mathrm{sec}^{-1}$ through an increase in the number of circulating protons seem promising.

Table V-2. Luminosity

\begin{tabular}{|c|c|c|}
\hline & Protons & Electrons \\
\hline $\begin{array}{l}\text { Energy } \\
\text { N/Bunch }\end{array}$ & $\begin{array}{l}2000 \\
6.9 \times 10^{10}\end{array}$ & $\begin{array}{l}\text { 10. } \mathrm{GeV} \\
8.5 \times 10^{10}\end{array}$ \\
\hline Bunch Frequency & \multicolumn{2}{|c|}{$17.7 \mathrm{MHz}$} \\
\hline $\begin{array}{l}\text { Current } \\
\text { Emittance (Horizontal) }\end{array}$ & 0.20 & $0.24 \mathrm{~mA}$ \\
\hline $\begin{array}{l}\text { Emittance (Horizontal) } \\
\text { Emittance (Vertical) } \\
\beta^{*}{ }^{\prime / *} \\
\text { Beam Size (Horizontal, rms) } \\
\text { Beam Size (Vertical, rms) }\end{array}$ & $\begin{array}{l}.01 \\
.01 \\
8.5 / 8.5 \\
0.12 \\
0.12\end{array}$ & $\begin{array}{l}.035 \mathrm{~mm}-\mathrm{mrad} \\
.016 \mathrm{~mm}-\mathrm{mrad} \\
.48 / .53 \mathrm{~m} \\
0.13 \mathrm{~mm} \\
0.09 \mathrm{~mm}\end{array}$ \\
\hline $\begin{array}{l}\text { Crossing Angle } \\
\text { Luminosity }\end{array}$ & \multicolumn{2}{|c|}{$6.2 \times 10^{31} \stackrel{0}{\mathrm{mrad}^{-2}} \mathrm{sec}^{-1}$} \\
\hline $\begin{array}{l}\text { Tune Shift (Horizontal) } \\
\text { Tune Shift (Vertical) }\end{array}$ & $\begin{array}{l}.0029 \\
.0040\end{array}$ & $\begin{array}{l}.027 \\
.030\end{array}$ \\
\hline
\end{tabular}




\section{B. Polarization}

Longitudinally polarized electrons are provided in each interaction region. The rotation from transverse (the equilibrium spin direction in the arcs) to longitudinal is produced by the six-magnet rotator shown in Fig. V-2. In the figure the angles refer to helicity precession through each dipole arising from the g-2 of the electron. The area between the two interaction regions is filled with the eight-magnet rotator shown in Fig. V-3. This series of magnets flips the helicity of the electron and guarantees that the spin has the proper orientation as it reenters the arc. This also results in the opposite helicity at the two interaction points. Reversing of the helicity from its naturally arising orientation can be carried out using resonant spin-flipping techniques pioneered at Novosibirsk.

Great care has been taken in integrating the rotators into the ring in such a way that the natural polarization is maintained by minimizing the effects of stochastic depolarization. The means by which polarization levels of greater than $80 \%$ can be maintained have been discussed in the Columbia $e^{-p}$ proposal (Fermilab Proposal 659) and will not be iterated here. It is sufficient to point out that the prescription described there has been followed and the result is a polarization level as calculated by the program "SLIM" of $80 \%$ and a polarization time of $15 \mathrm{~min}$. The polarization level is almost completely limited by the reverse bending introduced in the rotator magnets themselves. The spin level has been enhanced through the use of eight "kink" magnets with in the ring. These magnets are located in the special spin-betatron decoupling cells described below. The kink magnets are responsible for $14 \%$ of the total radiated power with in the ring. Without these magnets the polarization level would be $s 70 \%$ and the polarization time $\backsim 32 \mathrm{~min}$.

\section{Lattice}

The overall dimensions of the ring have been shown in Fig. V-1. The ring is a racetrack design consisting of two arcs of mean radius 153 $\mathrm{m}$, a straight section of length $349 \mathrm{~m}$ containing two interaction regions separated by $160.9 \mathrm{~m}$, and a $349 \mathrm{~m}$ off-side straight section which accommodates the $\mathrm{rf}$ and injection systems.

The guide field in the arcs is based on the standard FODO cell shown in Fig. V-4. The cell shown provides $90^{\circ}$ phase advance/cell. The required quadrupole gradient is $177 \mathrm{kG} / \mathrm{m}$ for the $60 \mathrm{~cm}$ long quadrupoles shown, and the magnetic bending field is $3.4 \mathrm{kG}$. In addition, each arc contains two of the special cells shown in Fig. V-5. These structures are used to decouple the spin and betatron motion (which are strongly coupled with in the FODO cell shown in Fig. V-4) by providing $360^{\circ}$ of betatron phase advance and only $180^{\circ}$ of spin phase advance each. The quadrupole magnets shown in Fig. V-5 are identical to those in the 


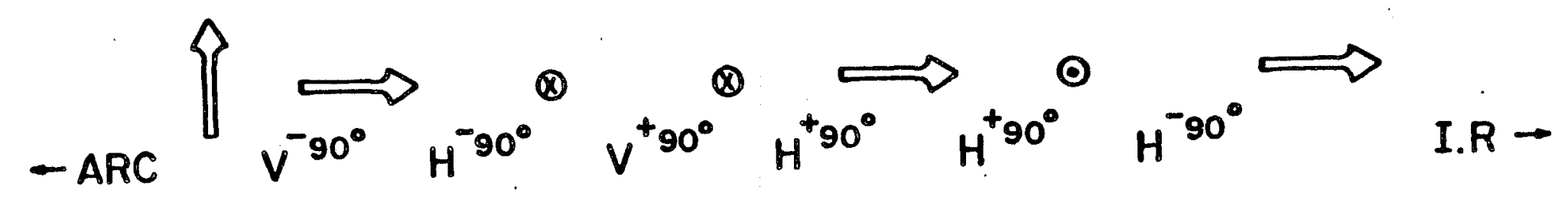

F1g. V-2. Action of the Spin Rotator.

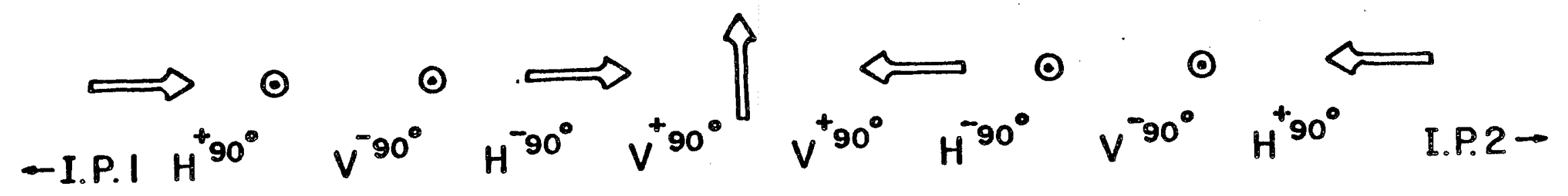

F1g. V-3. Transformation of the Spin Helicity between the two Interaction Points. 


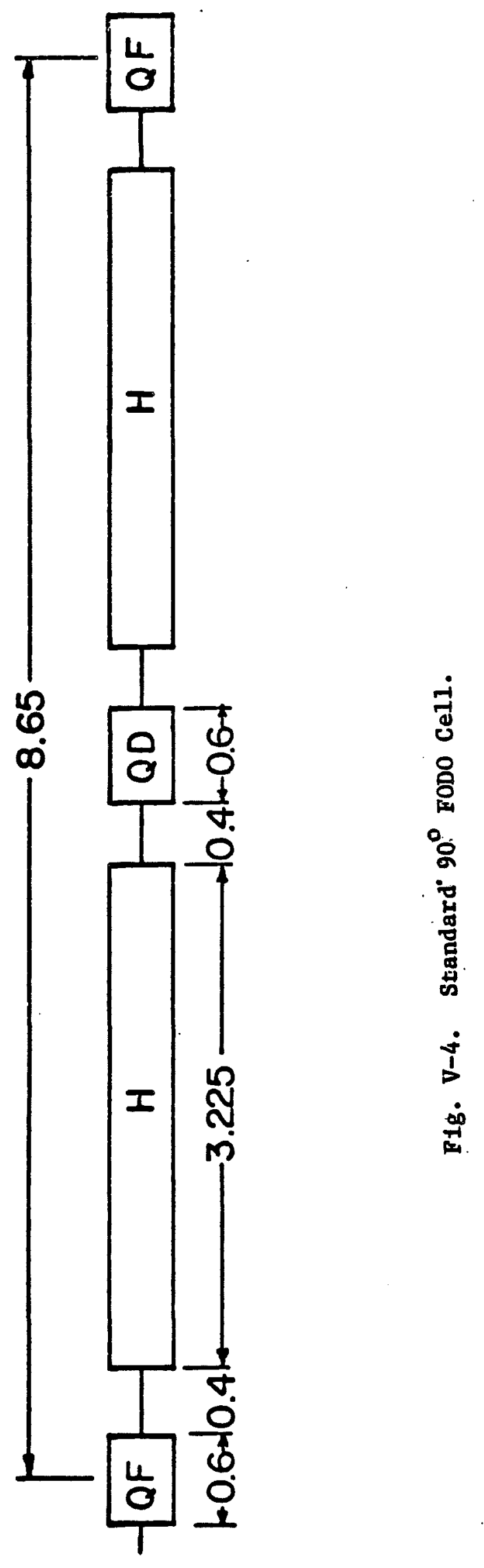




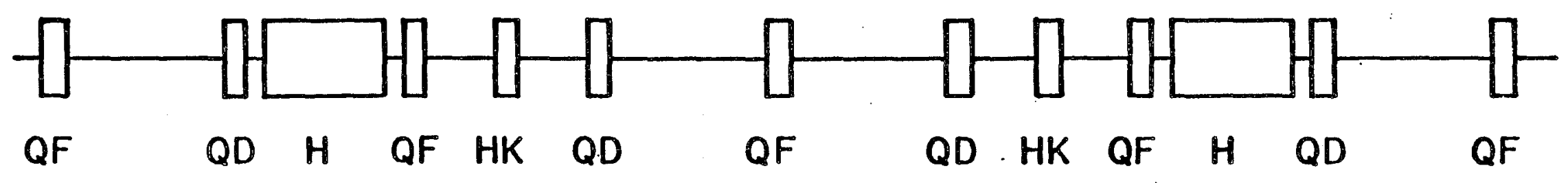

Fig. V-5. Spin-Betatron Decoup1ing Structure (HK:are kink magnets.run at $20 \mathrm{kG}$ for enhanced polarization). 

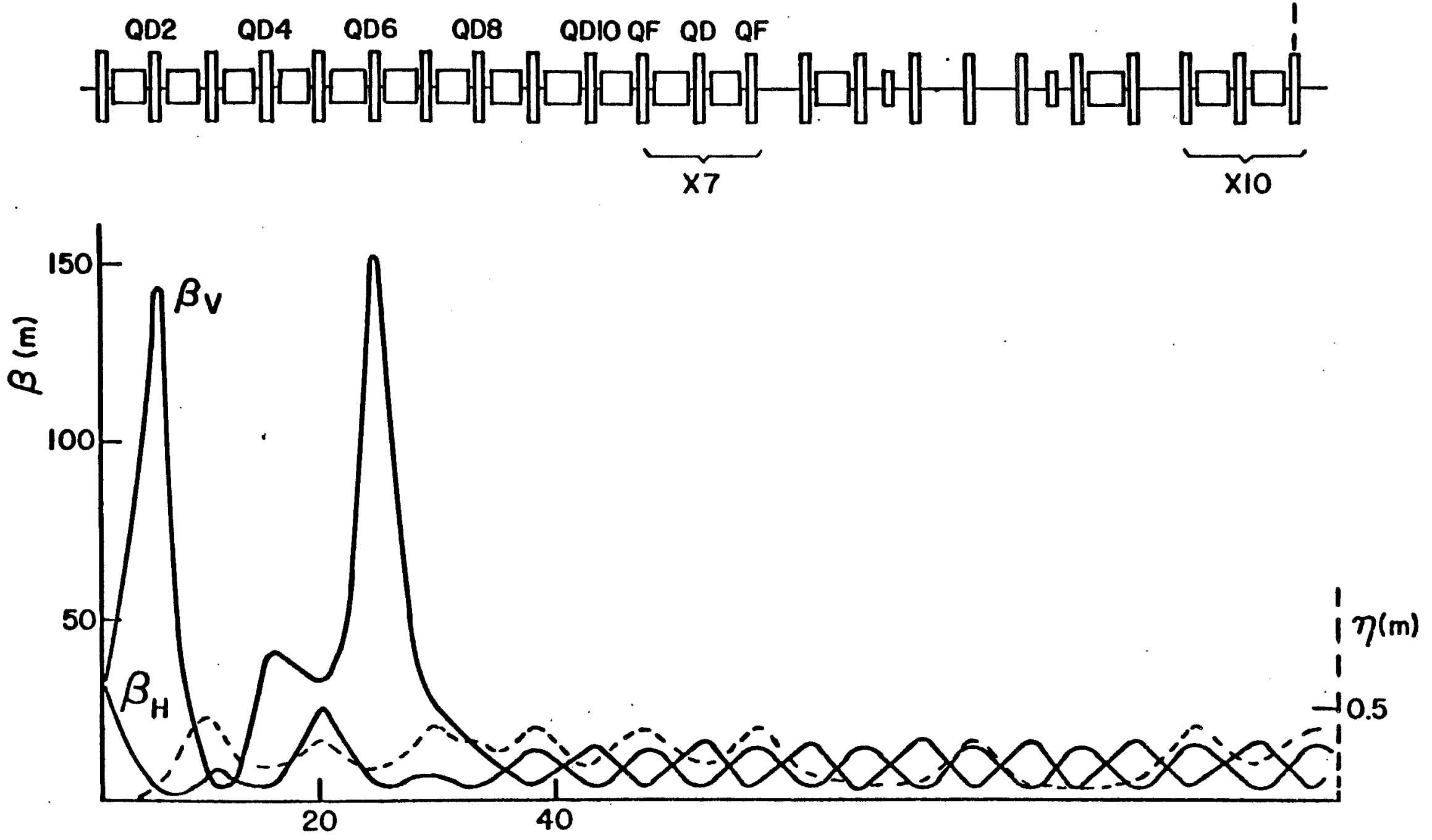

Fig. V-6. The Lattice through One-Half of an Arc. 
standard FODO cell. Two of the dipole magnets are also identical to those in the standard FODO cell. However, the other two are in igh-field (20 kG) "kink" magnets. The kink magnets have the same integrated kick as the standard dipole but because of their high fields enhance the polarization level and reduce the polarization time. In addition, because of their location in a region of low $H$ the "kink" magnets actually result in a reduction in both the horizontal and vertical beam emittance. The complete lattice functions through the arcs are shown in Fig. V-6. The last ten quadrupoles at each end of each are have their strengths adjusted to provide matching into the straight sections, dispersion suppression, and spin matching. Each arc is completely symmetric around its midpoint.

One half of the interaction region straight section is shown in Figs. $V-7 a$ and $V-7 b$. As stated above, the interaction region is designed to provide rotation of the electron polarization out of the transverse plane and onto the longitudinal axis. Shown in the figure are the location of both the electron and proton beamline elements. The separation between the two beams is $24 \mathrm{~cm}$ at the entrange to the first proton quadrupole $18.5 \mathrm{~m}$ from the interaction point, and is $60 \mathrm{~cm}$ at the electron dipole labelled V3. The electron beamline elements upstream of V2 are seen by the proton beam and are discussed in the following section.

The free space available to the experimenter is $\pm 5.0 \mathrm{~m}$ surrounding the interaction point. The beta function at the interaction point has a value of about $0.55 \mathrm{~m}$ in both dimensions. The horizontal dispersion and its derivative are both zero at the interaction point. The vertical dispersion is also zero although its derivative is 0.15 . The maximum value of beta through the interaction region is only $230 \mathrm{~m}$. The insertion satisifies all the lattice requirements for cancelling any contributions to stochastic depolarization. As a result of the vertical crossing shown in Fig. V-7, the plane of the electron ring is located $1.3 \mathrm{~m}$ above the plane of the DC. The two interaction regions are separated by $160.9 \mathrm{~m}$ ( 9.5 times the bunch separation) and have electrons of opposite helicity.

The of - side straight section is shown in Fig. V-8. It contains two $15 \mathrm{~m}$ and one $21 \mathrm{~m}$ long magnet-free regions of modest beta that can be used for $R F$ and injection.

The tune of the electron ring is close to 37 both horizontally and vertically. The high tune is a consequence of the need for a low emittance to match the electron beam to the proton beam. The tune can be controlled through adjustment of the quadrupole magnets in the off-side straight section and in the dispersion supressors. The natural chromaticity of the ring is -78 horizontally and -69 vertically. The chromaticity will be controlled by sextupole magnets placed immediately following each quadrupole in the arcs. The energy spread in the machine is $1.2 \times 10^{-3}$. 



Fig. V-7. Electron Ring through one Interaction Region (a) Side View, (b) Plan View. 

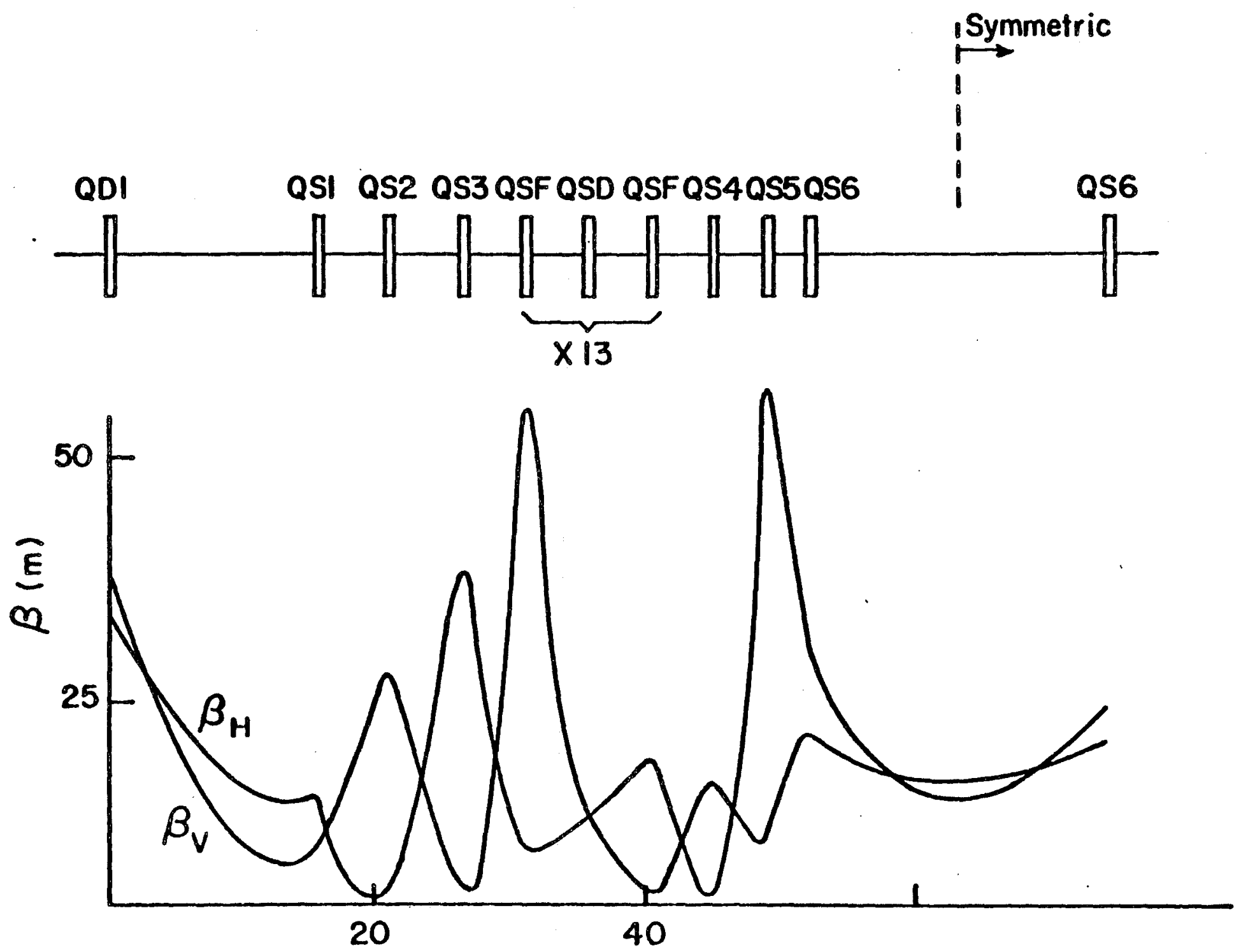

F1g. V-8. The Latice through the off-Side Straight Section. 


\section{Interaction Regions}

The interaction region shown in Fig. V-7 is designed to: 1) Maximize the luminosity while leaving sufficient free space for the detector; 2) keep the operation of the proton and electron rings as independent as possible; 3) minimize the amount of synchrotron radiation reaching both the detector and the superconducting elements of the DC.

The electrons and protons will come into collision in the utility straight section near the village. The proton-ring quadrupoles that produce the desired $\beta^{*}$ of $8.5 \mathrm{~m}$ with a free space of $\pm 18.5 \mathrm{~m}$ are shown in Fig. V-9. These quadrupoles are all run with field gradients less than or equal to those in the ares of the DC. The total horizontal betatron phase advance through the straight section is 1.04 wavelengths, eliminating the need for any changeover between ep and $\bar{p} p$ running.

The arrangement of dipole magnets in the electron ring is similar to that described in previous $e^{-p}$ proposals at Fermilab. The magnets Vo, V1, and V2 fill most of the distance from the interaction point to the first proton quadrupole. The magnet $v 0$ is a $\pm 5.0 \mathrm{~m}$ long, 67-G afr-core dipole which provides sufficient bending to insure that all radiation from the dipoles $V 1$ and $V 2$ can be shielded from the DC. VO, $\mathrm{V} 1$ and $\mathrm{V} 2$ bend the electron beam, $1 \mathrm{mrad}, 22.5 \mathrm{mrad}$, and 15 mrad respectively. The magnetic and radiation characteristics of these magnets are given in Table $\mathrm{V}-3$.

Table V-3. Interaction Region Magnets

\begin{tabular}{|c|c|c|c|c|c|c|}
\hline$\underline{\text { Name }}$ & 非 & $\underline{B(k G)}$ & $\underline{L(m)}$ & $\begin{array}{l}\text { Radiated } \\
\text { Power(W) }\end{array}$ & $\begin{array}{l}\text { Critical } \\
\text { Energy (kev) }\end{array}$ & $\begin{array}{c}\text { Photons per } \\
\text { Bunch }\end{array}$ \\
\hline vo & 4 & .067 & 5.0 & 6.8 & 0.4 & $1.7 \times 10^{10}$ \\
\hline v1 & 4 & 1.25 & 6.0 & $2.9 \times 10^{3}$ & 8.3 & $2.9 \times 10^{11}$ \\
\hline v2 & 4 & 1.25 & 4.0 & $1.9 \times 10^{3}$ & 8.3 & $2.6 \times 10^{11}$ \\
\hline v3 & 4 & 1.28 & 10.0 & $5.0 \times 10^{3}$ & 8.5 & $6.7 \times 10^{11}$ \\
\hline $\mathrm{HR}$ & 10 & 3.30 & 7.0 & $2.3 \times 10^{4}$ & 21.9 & $1.2 \times 10^{12}$ \\
\hline HRR & 2 & 4.98 & 4.6 & $3.5 \times 10^{4}$ & 33.1 & $1.2 \times 10^{12}$ \\
\hline VR & 4 & 3.30 & 7.0 & $2.3 \times 10^{4}$ & 21.9 & $1.2 \times 10^{12}$ \\
\hline VRR & 4 & 4.98 & 4.6 & $3.5 \times 10^{4}$ & 33.1 & $1.2 \times 10^{12}$ \\
\hline
\end{tabular}

It is unavoidable that some of the radiation from vo enter the $D C$ beampipe. To reduce this radiation as much as possible, a mask is placed just upstream of the first DC quadrupole. The proton beam size at" this point is $0.3 \mathrm{~mm}$ (rms). A 2-cm diameter hole provides clearance 

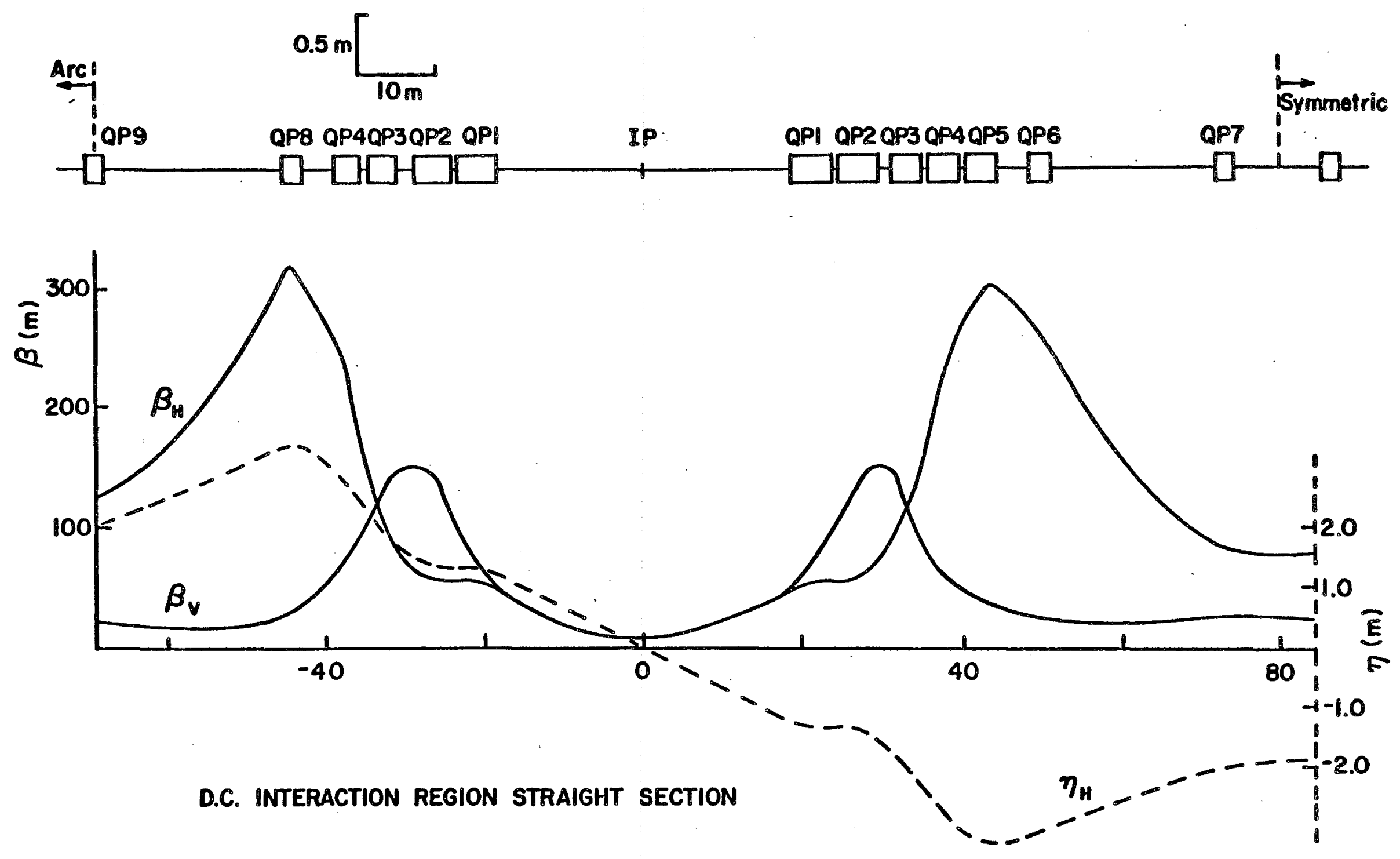

Fig。 $\mathbb{V}-9$ 。 
for the proton beam and subtends an angle of $\pm 0.54 \mathrm{mrad}$ at the interaction point. Thus, 55\% of the radiation produced in vo, i.e., 7.5 $W$, enters the DC beampipe and must be renoved by the refrigeration system.

The proton beam is allowed to pass fhrough the magnets vo and $V 1$. At the entrance to $V 2$ the two beams are separated by $8.8 \mathrm{~cm}$. This is sufficient space to make V2 a septum magnet. The perturbation to the proton beam caused by $V 0$ and $V 1$ can then be easily corrected by a pair of proton magnets located on the proton beam side of V2. The proton beam is also allowed to pass through the electron quadrupoles $Q 1$ and $Q 2$. Their effect is very small and easily compensated.

Because the bunch spacing in both the electron and proton beams is $16.94 \mathrm{~m}$, subsequent to the interaction of an electron and proton bunch at the interaction point there will be a passing of bunches $8.47 \mathrm{~m}$ away. Since the (vertical) beam separation at this point is $8.4 \mathrm{~mm}$, and the electron and proton beam sizes (rms) are $0.97 \mathrm{~mm}$ and $0.20 \mathrm{~mm}$ respectively, there will be no interaction at this point.

\section{E. Vacuum System}

The vacuum system is required to maintain an average pressure of $10^{-8}$ Torr in order to insure a beam lifetime due to beam-gas bremsstrainlung of greater than 20 hours. The beam lifetime due to this mechanism can be calculated in terms of the probability of an electron emitting a photon of energy greater than the energy aperture of cine machine as a result of an encounter with a gas molecule:

$$
\tau=\frac{1.58 \times 10^{-7} x_{o} /(P M)}{-\ln f-0.625+f+0.375 f^{2}}=\frac{1.58 \times 10^{-7} / P M}{\text { hours }}
$$

where $X_{0}$ is the radiation length $(\mathrm{g} / \mathrm{cm})$, $M$ is the molecular weight $(\mathrm{g})$, $P$ is the pressure (Torr), and $f$ is the ratio of the energy acsepliance to the beam energy. If we assume that the energy acceptance is determined by the RF system (see following section), and the residual gas is $75 \%$ H $+25 \% \mathrm{CO}$, we find

$$
\tau=\frac{22 \times 10^{-8}}{P(\text { Torr })} \text { hours }
$$

The main gas load arises from gas desorption from the vacuun chamber wall due to synchroton radiation. The linear power density in 
the arcs is $3.4 \mathrm{~kW} / \mathrm{m}$ and the gas load is estimated to be $1.2 \times 10^{13}$ molecules/sec/m assuming a desorption coefficient of $6 \times 10^{-6}$. This translates into a gas load of $3.5 \times 10^{-7} \mathrm{Tl} / \mathrm{sec}-\mathrm{m}$. A total pumping capacity of $35 \mathrm{l} / \mathrm{m}-\mathrm{sec}$ is then required for a vacuum of $10^{-8} \mathrm{~T}$. This capability is easily attained using distributed ion pumps of the CESR or PEP design. Commercially available ion pumps can also be installed throughout the ring to serve as holding pumps when the ragnetic fields in the dipoles are either low (as at injection) or turned off.

The vacuum chamber itself is somewhat smaller than the CESR or PEP chamber, although the design can be similar. The maximum required aperture outside the interaction region is $45 \mathrm{~mm}( \pm 15 \sigma)$. As such, a vacuum chamber $50 \mathrm{~mm}$ in diameter suffices. Bakeout procedures for reducing the desorption to the desired level have been developed at SLAC and PEP.

Pressures of about $10^{-10}$ Torr are needed in ine inceraction region to reduce backgrounds in the detector to colerable levels, and pumping and bakeout systerns adequate for this pressure will be provided.

F。

The design of the $r f$ system is influenced by a wide variety of considerations. These include a desire to provide a sufficient quantum lifetime, minimize the synchroton-oscillation tune, optimize the bunch length, maximize the shunt impedance, and minimize the effect on bean instabilities. In addition, the state of the available technology, cost, and ease of construction must be taken into account. It appears that the constraints imposed by the storage ring design and by construction and cost considerations can be met with a design based on the existing CESR system.

Table V-4 summarizes the characteristics of tire of system both for operation at $10 \mathrm{GeV}$ and at the injection energy of $5 \mathrm{GeV}$. The energy loss per turn due to synchrotron radiation at $10 \mathrm{GeV}$ is $13.2 \mathrm{MeV}$ (this does not include higher-order mode losses). The $r f$ voltage is chosen so that a quantum lifetime of 100 hours is obtained. It is also assuned that the voltage is programmed during tine injection and acceleration process such that the synchrotron tune remains constant. Note that the injection bunch length given assumes no bunoh lengthening. As is discussed later, bunch lengthening by a factor of two nay be expected at injection. 
Table V-4. RF System

\begin{tabular}{lll} 
& Peak & \multicolumn{2}{l}{ Injection } \\
Energy & 10.0 & $5.0 \mathrm{GeV}$ \\
Energy Loss/Turn & 13.2 & $0.8 \mathrm{MeV}$ \\
RF Voltage & 16.5 & $4.9 \mathrm{MV}$ \\
Frequency & 496. & $496 . \mathrm{MHz}$ \\
Harmonic Number & 2744 & 2744 \\
Synchrotron Tune & 0.017 & .017 \\
Bunch length & 1.09 & $0.54 \mathrm{~cm}$ \\
Energy Acceptance & 0.007 & .016 \\
Quantum Lifetime & 100 & $\infty$ \\
Power into Beam & 3.2 & $0.2 \mathrm{MW}$ \\
Cavity Shunt Impedence & 340 & $340 \mathrm{M} \Omega$ \\
Total Length & 12.6 & $12.6 \mathrm{~meters}$ \\
Total RF Power & 4.0 & $0.9 \mathrm{MW}$
\end{tabular}

Three CESR modules containing 14 cells each are used to provide the required $16.5 \mathrm{MV}$. The total length is then $12.6 \mathrm{~m}$. These cavities reside in the center of the off-side straight section. This region is designed to have a fairly low ( $s 12 \mathrm{~m}$ ) $\beta$ in an attempt to minimize the adverse effect of the rf cavities on beam stability. The total power requirement is $4.0 \mathrm{MW}$. This power can be supplied by eight $500 \mathrm{~kW}, 500$ MHz klystrons.

\section{G. Magnets}

The total number of magnetic elements in the ring is 702 . This includes 228 dipoles, 300 quadrupoles, and 174 sextupoles. All magnets are completely conventional in design. Their operating characteristics are given in Table V-5.

Table V-5. Magnet Characteristics

\begin{tabular}{cccccl} 
Magnet & Length & Strength & $\#$ & Aperture(HxV) & \multicolumn{1}{c}{ Comments } \\
$\begin{array}{ccccc}\text { Dipoles } \\
\text { Vo }\end{array}$ & $5.00 \mathrm{~m}$ & $.067 \mathrm{kG}$ & 4 & 6 & Air core \\
V1 & 6.00 & 1.25 & 4 & $6 \times 12$ & Interaction Region \\
V2 & 4.00 & 1.25 & 4 & $6 \times 12$ & Interaction (Septum) \\
V3 & 10.00 & 1.28 & 4 & $6 \times 12$ & Interaction \\
HR & 7.00 & 3.30 & 10 & $12 \times 6$ & Rotator \\
HRR & 4.63 & 4.98 & 2 & $12 \times 6$ & Rotator \\
VR & 7.00 & 3.30 & 4 & $6 \times 12$ & Rotator
\end{tabular}




$\begin{array}{lrrrrl}\text { VRR } & 4.63 & 4.98 & 4 & 12 \times 6 & \text { Rotator } \\ \text { H } & 3.23 & 3.38 & 184 & 12 \times 6 & \text { Standard Dipole } \\ \text { HR } & 0.55 & 20.00 & 8 & 12 \times 6 & \text { Kink Dipole }\end{array}$

Quadrupoles

$\begin{array}{llllll}\text { QF } & 0.60 & 178 . \mathrm{kG} / \mathrm{m} & 86 & 6 . \mathrm{cm} & \text { Standard quad. } \\ \text { QD } & 0.60 & -178 . & 84 & 6 . & \text { Standard Quad. } \\ \text { Q } & 0.60 & 222 .(\mathrm{Max}) & 40 & 6 . & \text { Dispersion Supr. } \\ \text { Q } & 0.60 & 196 .(\mathrm{Max}) & 66 & 6 . & \text { Off-side Straight } \\ \text { Q1 } & 0.80 & -177 . & 4 & 9 . & \text { Ineraction Region } \\ \text { Q2 } & 0.80 & -177 . & 4 & 9 . & \text { Interaction Region } \\ \text { Q3 } & 0.80 & 151 . & 4 & 7.5 & \text { Interaction Region } \\ \text { Q4 } & 0.80 & -18 . & 4 & 7.5 & \text { Interaction Region } \\ \text { Q5 } & 0.80 & -33 . & 4 & 7.5 & \text { Interaction Region } \\ \text { Q6 } & 0.80 & 36 . & 4 & 7.5 & \text { Interaction Region }\end{array}$

Sextupoles

$\begin{array}{lllll}S F & 0.25 & 0.31 \mathrm{kG} / \mathrm{cm}^{2} & 86 & 6 . \\ \text { SD } & 0.25 & 0.40 & 88 & 6 .\end{array}$

The arcs of the ring contain 184 standard and 8 "kink" dipoles. These magnets have lengths of $3.225 \mathrm{~m}$ and $0.545 \mathrm{~m}$ respectively. The field strengths are $3.38 \mathrm{~kg}$ and $20.00 \mathrm{~kg}$. Since the maximum beam size through the arcs $( \pm 15 \sigma)$ is $32.5 \mathrm{~mm}$ horizontally and $45 \mathrm{~mm}$ vertically. a ragnet aperture of $120 \mathrm{~mm}$ by $60 \mathrm{~mm}$ is adequate for containing the beam within the beampipe. We plan that these will be C-magnets in order to provide ease of access to the vacuum chamber. The remaining dipoles consist of 20 rotator magnets and 16 interaction-region dipoles. The only exceptional magnets in this group are the 67-G air-core dipole at the interaction point and the septum magnet $V 2$.

The 275 quadrupole magnets in the arcs and off-side straight section are each $60 \mathrm{~cm}$ long with a bore diameter of $60 \mathrm{~mm}$. The field gradients in the standard quadrupoles are $178 \mathrm{kG} / \mathrm{m}$ and in the disperson-suppression region range up to $222 \mathrm{~kg} / \mathrm{m}$. This results in a maximum pole tip field of $6.7 \mathrm{kG}$ for the given aperture. The remaining 24 quadrupoles occupy the two interaction regions. These quadrupoles are all $80 \mathrm{~cm}$ in lengti. The only difficult ones are the quadrupoles $Q 1$ and Q2 closest to the interaction region. These magnets require an aperture of $90 \mathrm{~mm}$ and a field of $177 \mathrm{kG} / \mathrm{m}$ (in Q1). The corresponding Cield at tire pole tip is $8.0 \mathrm{kG}$.

Sextupoles are distributed throughout the ring io sorrect the natural chromaticity of the machine. If two families are dised, the strengths are modest-- $0.31 \mathrm{kG} / \mathrm{cm}^{2}$ and $0.40 \mathrm{kG} / \mathrm{cm}^{2}$ for a length of $25 \mathrm{~cm}$. There are 154 such sextupoles in the ring and they, like the quadripoles, require an aperture of $60 \mathrm{~mm}$. The fields at the pole tips are then $1.4 \mathrm{kG}$ and $1.8 \mathrm{kG}$ respectively. 


\section{H. Injection}

The choice of injection energy into the $10-\mathrm{GeV}$ storage ring is made on the basis of the beam-stability characteristics of the ring when operating at the injection energy. We believe that it is single-beam instabilities that provide the ultimate limit on the amount of charge that can be injected into the storage ring at a given energy. We have examined three sorts of instabilities: 1) The beam lifetime due to Touschek scattering; 2) the current threshold for the onset of head-tail turbulence; and 3) the expected degree of bunch lengthening. Estimates are based on previous experience at SPEAR, PEP, and DORIS. We conclude that an injection energy of $5 \mathrm{GeV}$ is the minimum for which we can rellably expect to be able to fill the 10-GeV ring with the required number of electrons. At this energy, the Touschek lifetime is calculated to be 1.1 hours and the current limit due to the head-tail effect to be $3.5 \mathrm{~mA} /$ bunch in the absence of bunch lengthening. However, it is expected that bunch lengthening will occur at injection resulting in bunch lengths of $1 \mathrm{~cm}$ rather than $0.5 \mathrm{~cm}$ given by the RF system.

The injection system consists of an 120-MeV linac (followed by an additional $80 \mathrm{MeV}$ for positron injection) followed by a rapid-cycling $(15 \cdot \mathrm{Hz}) 5-\mathrm{GeV}$ booster synchrotron. Some fraction of the Mark III linac from HEPL may be available for use as a source of electrons and positrons for the booster ring. The Mark III linac was originally built as a prototype for SLAC and as such has an identical accelerating structure. The linac has been out of commission for several years, but is being revived now for use in a free electron laser project. This project requires only half the thirty 10-ft sections of the linac. The acquisition of five of these sections would provide an ideal injector into the booster. When driven by a modern SLAC klystron, each 10-ft section is capable of supplying $40 \mathrm{MeV}$ of acceleration. The total available energy of $200 \mathrm{MeV}$ is adequate for both electron and positron injection.

The booster ring has a circumference of $338.7 \mathrm{~m}$ ( 20 bunches). The electrons (or positrons) are injected directly into the booster at 80 $\mathrm{MeV}$ every $67 \mathrm{msec}$. They are then accelerated up to $5 \mathrm{GeV}$. Since the (transverse) damping time in the booster ring is $16 \mathrm{msec}$, the beam is nearly completely damped when it is extracted from the booster and injected into the $10-\mathrm{GeV}$ ring.

Electron and positron accumulation takes place in the storage ring. Bunches from the booster are stacked in transverse phase space and moved onto the central orbit by radiation damping ( $\tau=67 \mathrm{msec})$. The electron filling time for a system based on such a linac-booster injection system is calculated to be $12 \mathrm{sec}$ for an electron-gun current of $2 \mathrm{~A}$ and the linac operating without either a prebuncher or buncher. The positron accumulation time has been calculated using the shower-generation program "EGS", assuming a booster admittance of $50 \pi \times 10^{-6} \mathrm{~m}$ for $\Delta p / p= \pm 4 \%$ an electron-gun current of $5 \mathrm{~A}$, and the use of both a prebuncher and buncher. The resultant calculated filling time is $125 \mathrm{sec}$. 


\section{Future Upgrading}

Future extension of the $e^{-} p$ center of mass energy into the range $\sqrt{\mathrm{s}}>500 \mathrm{GeV}$ is possible with an electron ring residing within the DC tunnel, using the storage ring described here as an injector. The optimum energy for an electron ring within the DC tunnel lies in the range $35.40 \mathrm{GeV}$. At $40 \mathrm{GeV}$, the radiated energy is $188 \mathrm{MeV} /$ turn and the radiated power is $28 \mathrm{MW}$ for a circulating current of $150 \mathrm{~mA}$. The luminosity attainable is about $1 \times 10^{32} \mathrm{~cm}^{-2} \mathrm{sec}^{-1}$ under assumptions similar to those given earlier. A possible parameter list for such a ring is given in Table V-6. An injection energy of $15 \mathrm{GeV}$ is needed to ensure beam stability at injection.

\section{Table V-6. $40 \mathrm{GeV}$ e-Ring Parameters}

$\begin{array}{lll}\text { Energy } & 40 & \mathrm{GeV} \\ \text { Injection Energy } & 15 & \mathrm{GeV} \\ \text { Circumference } & 13210 \mathrm{~m} \\ \text { Bunches } & 780 & \\ \text { Electrons/Bunch } & 5.3 \times 10^{10}(150 \mathrm{~mA}) \\ \text { Tune } & 78 & \\ \text { Dipole Field } & 1.1 \mathrm{kG} & \\ \text { Number of Dipoles } & 624 & \\ \text { Dipole Length } & 12.1 & \mathrm{~m} \\ \text { Cell Length }\left(90^{\circ}\right) & 30.2 \mathrm{~m} \\ \text { Energy Loss/Turn } & 188 & \mathrm{~m} \\ \text { Luminosity } & 1 \times 10^{32} \mathrm{~cm}^{-2} \mathrm{sec}^{-1}\end{array}$

The $10 \mathrm{GeV}$ storage ring described here can be operated as a $15 \mathrm{GeV}$ synchrotron for injection into a higher energy machine by raising the field in the bending magnets to $5.0 \mathrm{kG}$, removing the rotator magnets, and installing additional $r f$. At $15 \mathrm{GeV}$ the radiated energy is 45 $\mathrm{MeV} /$ turn so a voltage of $s 50 \mathrm{MV}$ is required. However very little power is required because of the small circulating current. The beam emittance will increase (compared to $10 \mathrm{GeV}$ operation) due to the increased energy and lowered tune if the quadrupole fields remain fixed. But removal of the rotator magnets nearly compensates for these effects and results in a horizontal emittance which is only $40 \%$ higher than at $10 \mathrm{GeV}$, and a vertical emittance which is of course much less. As a consequence all apertures are completely adequate for operation at 15 GeV.

Injection would proceed as described previously with accumulation taking place within the storage ring and the injection system cycling at $15 \mathrm{~Hz}$. Since the ratio of the circumference of the $40 \mathrm{GeV}$ ring to the 
$15 \mathrm{GeV}$ booster is approximately 8:1, filling times of eight (or less if a smaller circulating current is required) times those given for the 10 GeV ring would be expected. 


\section{A. Site geology: topographic and subsurface features}

Considerable information exists on the geologic features and subsurface conditions on the site, although it is clear that a large number of test borings will necessarily precede a truly accurate cost estimate for tunnel and enclosure construction. Here we briefly sketch the state of known information about the portions of the site which are relevant for construction of the DC.

After the Fermilab site was selected in 1966, 67 widely distributed test borings were drilled by the U.S. Amy Corps of Engineers during the period January through September of 1967. Analyses of core samples were carried out to determine engineering properties. The Illinois state Geological Survey developed the pattern and history of the subsurface stratigraphy. Much of the discussion of the next section is based on this site-wide study.

During 1968, numerous additional borings were made by Soil Testing Services, Inc., of Northbrook, Illinois, primarily at locations selected for the principal laboratory facilities. When combined with the corps of Engineers data, these data give a rather detailed picture of subsurface conditions along the perimeter of the Main Bing enclosure. Predictions of enclosure settlement were based on this information. The measured subsidence ( 1 inch) was only slightly larger than predicted, and in any case did not impede commissioning or operation of the Main Ring.

Like most of the surrounding several hundred thousand square miles of the Central Lowlands, the Fermilab site is rather flat; maximum relief over the 6800 acres is only about $70 \mathrm{ft}$. Along the western boundary, a gentle rise of some $40 \mathrm{ft}$ marks the Minooka Moraine, one of a number of terminal moraines which are roughly concentric with the lower end of Lake Michigan. Just to the east of the site, the Valparaiso Moraine defines a similar line of slightly higher ground. Generally speaking, the lowest surface elvations are to be found in the southeastern portions of the Laboratory, where there are substantial swampy areas. These are drained by Ferry Creek, which crosses the eastern site boundary between the Village and the southeastern corner of the site, and eventually finds its way to the West Branch of the DuPage River.

The bedrock surface is also relatively flat, decreasing in elevation gradually toward the east, and is encountered typically at depths of between 60 and $100 \mathrm{ft}$ below ground level. This rock is dolomite of the Silurian period. The level of the permanent water table lies within this rock layer, which is laterally permeable and, according to the Illinois State Water Survey, forms an important aquifer. 
The strata between ground surface and bedrock reflect the advance and retreat of "recent" glaciation. Whatever materials overlaid the bedrock in the hundreds of millions of years between Silurian and Pleistocene times have vanished. The glacial deposits have been grouped by the Illinois State Geological Survey into five stratigraphic units, which in descending order are characterized as follows:

Unit A. The surface layer contains silts and sands deposited from lakes or streams, and silts brought in by the wind. The wind-borne materials cover much of the site with a thickness of a few feet, and are mineralogically distinct from the water-deposited materials, which are similar to the underlying strata.

Unit B. This is a glacial till composed primarily of silt and clay, with some sand and gravel. It is much firmer material than Unit A and relatively impenetrable to water. The Minooka Moraine is composed of this till.

Unit $C$. This layer is a mixture of sand, silt, and gravel. Little clay is present; thus this unit is relatively incompressible.

Unit D. This stratum has the higisest clay content (up to $75 \%$ ) of the various tills. It has higher moisture content and lower density than the till of Unit $B$. It is possible that this layer is associated with an earlier glaciation than the most recent (Wisconsinian) ice age.

Unit E. This is a sandy and silty till containing deposits of sand and gravel which rests directly on bedrock.

Not all of the above units need necessarily be found in a particular boring, and the strata are by no means as flat as either bedrock or the surface of the ground. Fig. VI-la illustrates the distribution of the various layers along Wilson Road from one site boundary to the other. Note that Unit $A$ is absent in the easternmost portion, near the Minooka Moraine, while both Units $D$ and $E$ vanish in a small region near the center of the figure. Notice that the region marked on the figure is reasonably close to the DC and may give a very rough indication of the subsurface material to be encountered.

With the exception of Unit $A$, all of the strata have been subjected to . glacial overburden and are well consolidated. All of these subsurface layers, if undisturbed, are suitable foundation materials for accelerator enclosures, and so long as the stress which propagates downward from these enclosures, other structures, and shielding does not exceed that imposed by the glaciation, settlement wil.l be within reasonable bounds.

The Main Ring tunnel floor elevation of $722.5 \mathrm{ft}$ above mean sea level was high enough to impose no unusual difficulties to a cut-and-fill operation, yet low enough so that the foundation material would be that of stratigraphic Unit B. This level was also sufficiently low that subsequent covering of the enclosure by the shielding berm 
would not increase stresses in the subsurface layers exceeding those previously applied by the glacial burden.

The concrete floor of the enclosure rests directly on the till. In those places where unconsolidated materials were found at bine base of the out (For example, a low point in Unit $A$ ), they were repiaced by lean concrete. Similarly, in the event of over-excavation, lean concrete was used to restore the foundation to the proper level. The enclosure floor was formed and poured in place. The precast concrete hoops (the sides and ceiling of the tunnel) were fabricated on site and placed quickiy, yet accurately, on the foundation. The construction sequence therefore consisted of a limited number of steps and proceeded rapidly and smoothly. It should be noted that where more intricate structures were necessary, as in the service buildings, their access-ways to the tunnel, and the Transfer Hall, these features were slow to complete and more costly.

How well the elevation chosen for the Main Ring enclosure conforms with the subsurface conditions is illustrated in Fig. VI-2. Here, data from the test-boring logs have been used to reconstruct the location of the various stratigraphic layers throughout the entire perimeter of the main accelerator. Note that the 722.5 ft elevation indeed lies within Init B with little exception. Though Unit $C$ is less compressible than $B$, it not only occurs at too low an elevation for the enclosure, but it rests in turn on the most compressible of the strata, Unit $D$.

Again the regions indicated in Fig. VI-2 lie quite close to the DC ring, and again give a rough indication of the conditions to be encountered.

Thus in the north and south portions of the DC ring, the known soil conditions appear in general to be satisfactory, typical of those encountered in Main Ring tunnel construction. Additional test borings have been made in other regions to be traversed by the $D C$.

\section{B. Special problems}

Most of the ring traverses quite level terrain with mean elevation of about 740 ft. Nevertheless there are exceptional regions which require special consideration. Perusal of Fig. VI-3 shows that in the northwest quadrant the tunnel floor is about $70 \mathrm{ft}$ below grade as it penetrates the Minooka Moraine (hereafter Mt. Minooka). The ep collider rests in the midst of Lake Law. The southwest collision region rests on the banks of Indian Creek. These and other apparent problems will be briefly discussed below:

1. Lake Law: This lake is artificial. It will be drained and reconfigured into cooling ponds for the $\cong 18 \mathrm{MW}$ of power dissipated in that area by the electron ring and the ep experimental apparatus. A large number of soil borings exist in that area. The results indicate good foundation conditions. 

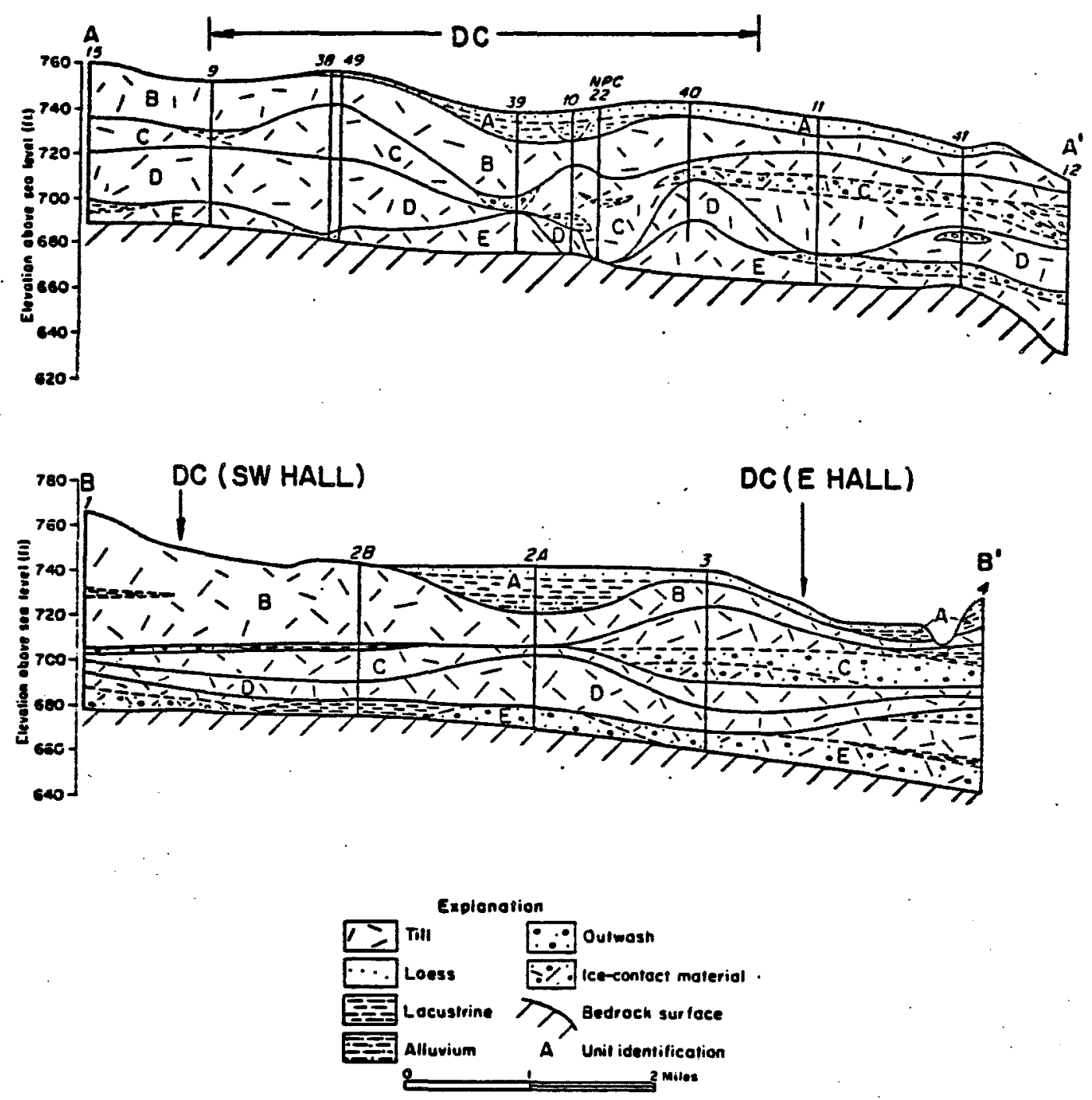

Fig. VI-1. Geological Profile (a) along Wilson Road and (b) along a line from the SW Collision Hall to the E Colliston Hall. 

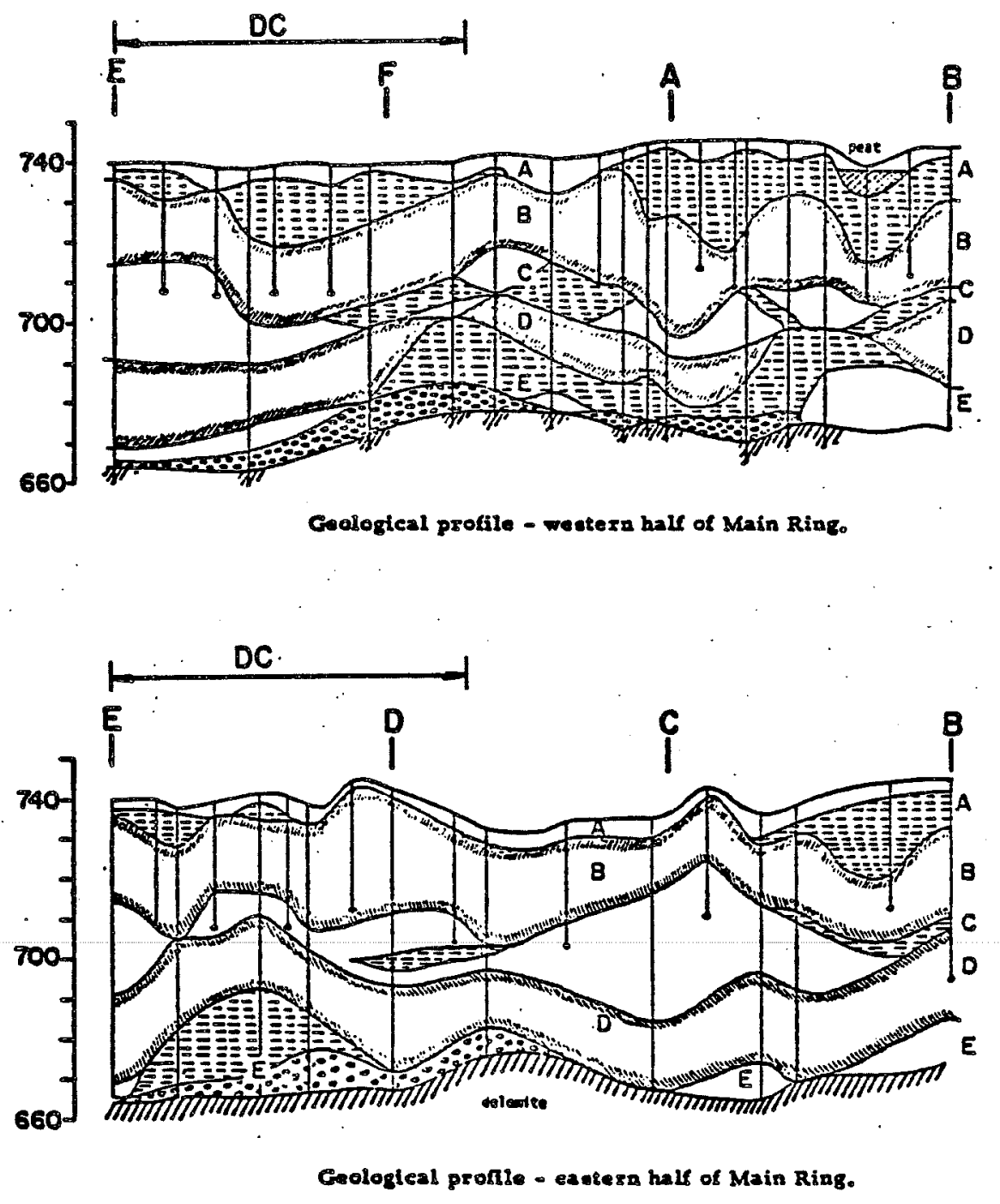

F18. VI-2。 


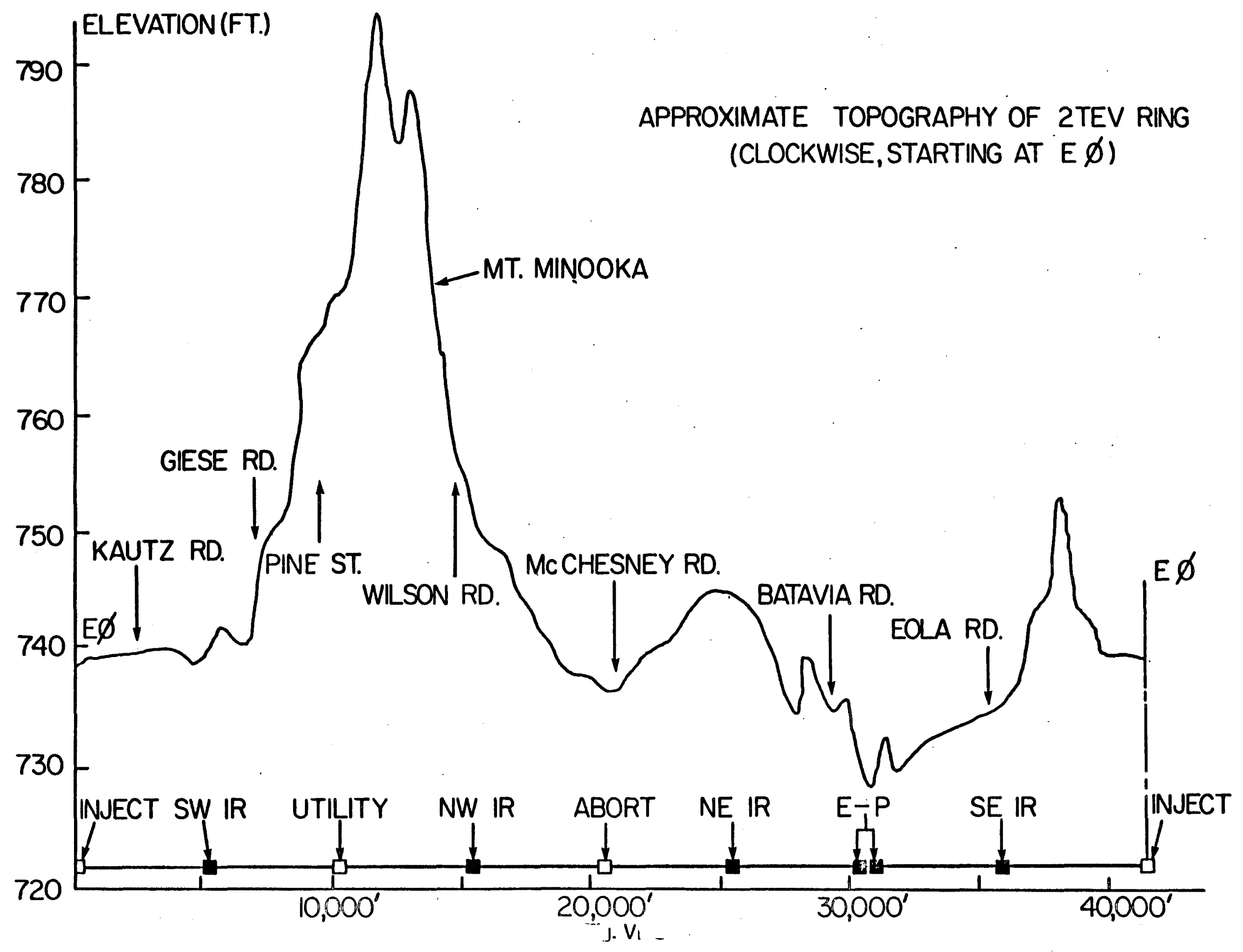


2. Indian Creek: This watercourse will need to be diverted and means provided to convey creek water past the berm. Control of this watercourse is not expected to be difficult or costly. Again, since the crossing is near the RF station and the southwest collision hal1, a cooling pond for that area is needed and can be incorporated into the system.

The site of the collision hall is $200 \mathrm{ft}$ from the creek. While there are few soil borings in this portion of the site, there is no reason to expect difficult soil conditions. (See in particular Fig. VI- ib)

3. Neutrino area: The accelerator tunnel passes just north of Casey's Pond, and again the drainage from the north into Casey's Pond must cross the berm. The volume is small and this should cause no problem. The accelerator tunnel also passes just north of the Muon Laboratory (floor elevation $719 \mathrm{ft}$ ), to be constructed well before the DC. The two facilities are mutually compatible.

4. Mt. Minooka: Given a tunnel of order the size of the Main Ring enclosure, it is very likely that soft tunnelling will be economically favored over cut-and-fill when the depth of cut exceeds 30 to $40 \mathrm{ft}$. This condition only exists in the northwest portion of the ring, roughly from the Pine Street crossing to the Wilson Road crossing, a distance of about $6100 \mathrm{ft}$. Soft tunnelling has become common in recent years and thus would be especially attractive in this portion of the DC tunnel. An $8 \mathrm{ft}$ nominal diameter is a minimum size, and tunnelling machines for horseshoe-shaped tunnels are available. There exist several experienced soft-tunnelling contractors in the Chicago area.

Evidently much additional information must be collected before an accurate cost estimate could be developed. On the basis of the one soil boring extant for this portion of the are, the soil between 720 and 730 ft seems to be well-consolidated clay (Unit $B$ ) which should be suitable for tunneliing. Bedrock can be presumed to be between elevations 690 and $700 \mathrm{ft}$. Thus it appears likely that the cost penalty for this portion of the tunnel is not severe. For the purposes of this study, we assume conservatively that it is $\$ 3 M$. (The nominal cost of $6100 \mathrm{ft}$ of standard $8 \mathrm{ft} \times 9 \mathrm{ft}$ tunnel is about $\$ 5.8 \mathrm{M}$. ) Costs of (soft) tunnels of $8 \mathrm{ft}$ diameter constructed in the Chicago area are quoted as low as $\$ 700 / \mathrm{ft}$.

\section{Tunnel}

There is considerable recent experience for tunnel construction at Fermilab because of the TeV II construction. We choose for this study a spacious $8 \mathrm{ft} \times 9 \mathrm{ft}$ tunnel of rectangular-cross-section. It is composed of precast concrete "Un arches set upon a cast-in-place base slab. The tunnel components include, in addition to the string of magnets, helium and nitrogen headers, conventional AC power, miscellaneous instrumentation and control cables, and lighting. 


\section{Experimental areas}

The detailed specification and design of experimental areas most naturally follows an examination in a summer study or similar review of the physics opportunities of the DC by the community of experimenters that plans to use 1t. From the experience already obtained at the CERN collider and from plans at $T e V I$, it is expected that a variety of experimental areas will be required. For planning purposes, it is assumed that all four collision regions will have experimental areas and service areas built up around them. It is extremely likely that more than one large general-purpose detector like UA 1,2 or CDF will be needed. To accommodate them, two of the areas are chosen to be of the same scale as the BO experimental area of TeV I. Several smaller experiments ran successfully at CERN and others are proposed for the DO region of $\mathrm{TeV} I$. They can be mounted in a smaller area, of the scale of DO. Depending on topography, it may be possible to have a relatively large floor area for this "small experiment" region, but the beam line must be close to the floor, say 4 meters or less. In such an area, several experiments could run simultaneously or be able to roll in and out easily and at small extra cost. The 4th area is projected to be larger than $B O$ to be able to accommodate a very large detector that is about 50\% larger than $\mathrm{CDF}$.

The e-p collider requires two collision areas that reside along a common long straight section. For estimation purposes, it is assumed that the two areas are similar in size to the BO collision hall.

For any area, initially only the collision hall need be constructed. It could be shielded and the assembly area constructed at a later date. It is assumed in this proposal that if the $\bar{p} \bar{p}$ option is chosen (with the decision to be made in about FY86) then the ep assembly areas would wait, and if the ep option were chosen, the $p \bar{p}$ assembly areas would wait.

The medium experimental areas are chosen to be identical to the present BO experimental area of TeV I. There is a collision hall 100 ' long composed of a 50' $\times 50^{\prime}$ central section with two $25^{\prime} \times 35^{\prime}$ end pieces forming forward and backward arms. The beam height is $17^{\prime}$ above the floor in the central part. The collision hall is connected to an outside assembly area by a $35^{\prime} \times 35^{\prime}$ tunnel which is blocked with a shielding door during operation. At the end of the tunnel is a $751 \times 100$ ' assembly area at the same elevation as the collision hall. Over this assembly area is a $250^{\prime} \times 75^{\prime}$ ' high bay providing surface level support areas and a 50-ton overhead crane. Immediately adjacent to this assembly area is a three level mezzanine complex $25^{\prime} \times 250^{\prime}$ providing counting rooms, of fices, and shop space.

The large experimental area is designed to accommodate a larger detector than the present design of CDF. The collision hall is 140. long and composed of a $60^{\prime} \times 60^{\prime}$ central area having a $20^{\prime}$ beam height with $40^{\prime} \times 40^{\prime}$ forward/backward arms. The tunnel connecting the collision hall to the assembly area is $40^{\prime} \times 40^{\prime}$. The assembly area is also larger; 
$75^{\prime} \times 150^{\prime}$ at the lower level, and $100^{\prime} \times 300^{\prime}$ at the upper level. The same general design was adopted as for the medium area.

The small experiment area is designed to be able to alternately switch two experiments. The collision hall itself is chosen to be of uniform cross section and long $-35^{\prime} \times 150^{\prime}$, with a beam height of $12^{\circ}$, in order to emphasize "forward" physics. This is connected via a $25^{\circ} \times 25^{\prime}$ " tunnel to an assembly area. This assembly area is designed to be used by two groups. The lower level is $50 \times 150^{\prime}$ with the intention that one group uses one end for assembly while the other group uses the other end. This building would have two 20 ton cranes. The mezzanine area would be split and have two sets of counting rooms, etc.

The two e-p areas would be similar to the medium expertment area with the downstream arm lopped of the collision hall, along with a smaller assembly building. The collision hall would be $50^{\circ} \times 50^{\prime}$ with a $25^{\circ} \times 35^{\prime}$ hadron arm extension. The beam height in the central area would be $17^{\circ}$. A standard $35^{\prime} \times 35^{\circ}$ tunnel would connect to the assembly area having a $75^{\prime} \times 75^{\prime}$ lower level and a $150^{\prime} \times 100^{\prime}$ upper level. Standard mezzanines would supply the counting room, office, and shop space. Again only the collision halls of the ep areas need be constructed. The tunnel can be sealed and the assembly areas constructed later.

The collision hall layouts are shown in Figs. VI-4 to VI-7, and Table VI-2 provides a list of parameters.

\section{Table VI-2: Parameters of Collision Halls}

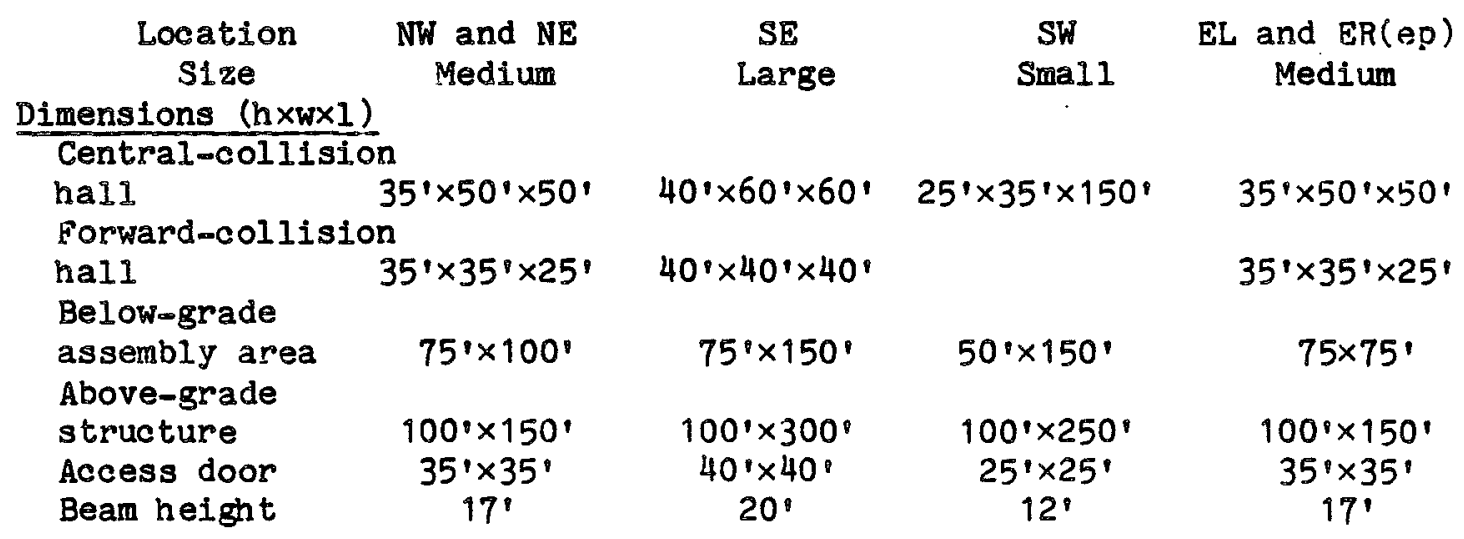

E. Miscellaneous enclosures

Unlike the Main Ring, there are four major access points to the DC, through the collision halls. Vehicle accesses for aagnets and other equipment to be brought into the tunnel will be provided via these collision halls, which will comprise the principal means of personnel access to the ring as well. 
Medium Experiment Areas; All elevations assume beam elevation $720^{\circ}$ Ground level 740'.

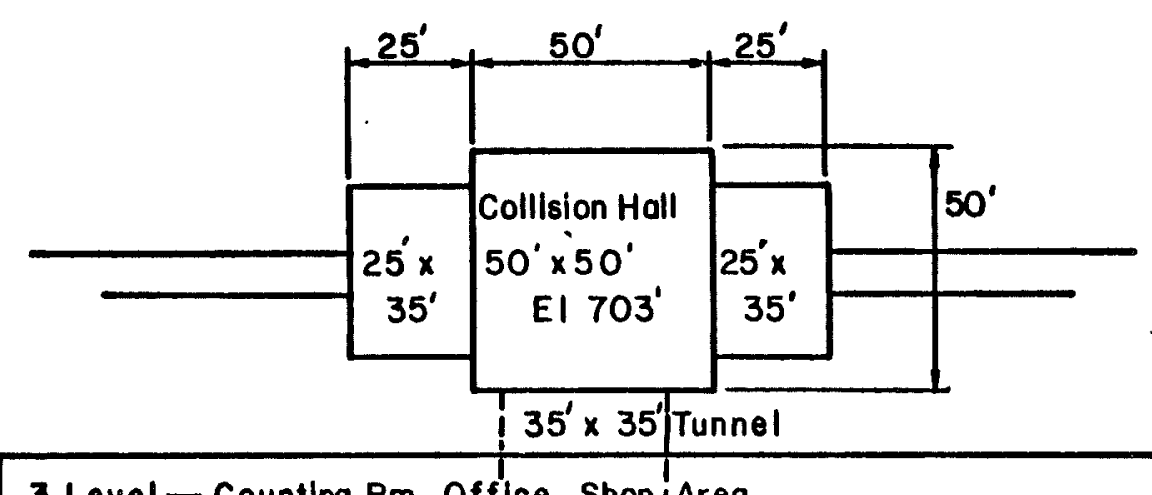

\begin{tabular}{|c|c|c|}
\hline 3 Level-C & , Office, shop í & \\
\hline $\begin{array}{l}\text { Assembly } \\
\text { Support } \\
75^{\prime} \times 75^{\prime} \\
\text {.EI. } 740^{\prime}\end{array}$ & $\begin{array}{l}\text { Assembly Area } \\
75^{\prime} \times 100^{\prime} \\
\text { El. } 703^{\prime}\end{array}$ & $\begin{array}{l}\text { Assembly } \\
\text { Support } \\
75^{\prime} \times 75^{\prime} \\
\text { EI. } 740^{\prime}\end{array}$ \\
\hline $75^{\prime}$ & $100^{\prime}$ & $75^{\prime}$ \\
\hline
\end{tabular}

$17^{\prime}$ Beam Height $35^{\prime} \times 35^{\prime}$ Door

Fig. VI-4 
Large Experiment Area: All elevations assume beam elevation 720;

Ground level $740^{\circ}$.

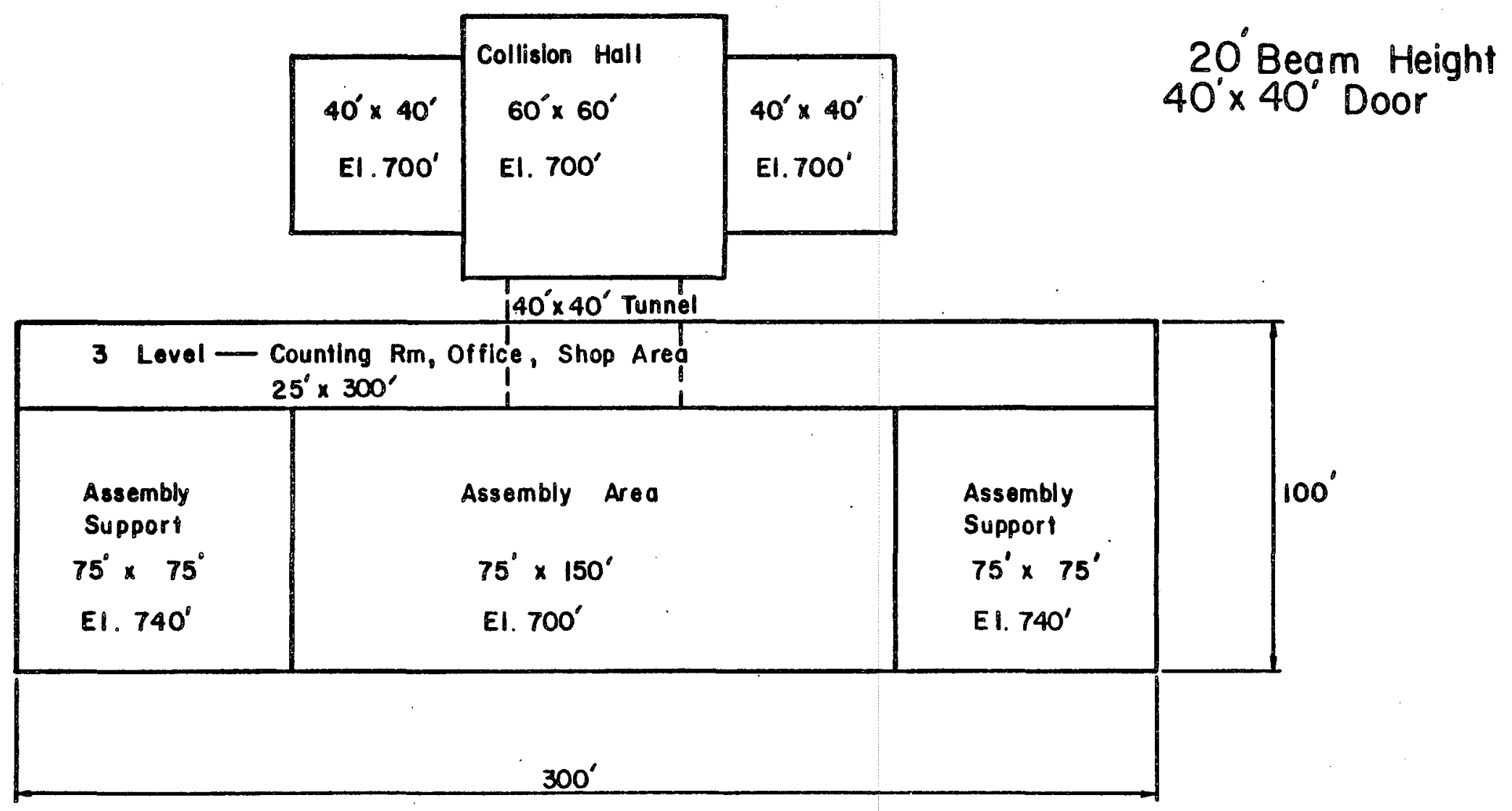

Fig. VI-5 
Small Experiment Area: Switch for two experiments, All elevations assume beam elevation 720 .'

Ground level 740'.



Fig. VI-6 
e-p Experiment Areas; All elevations assume beam elevation $720^{\prime}$ Ground level 740.'

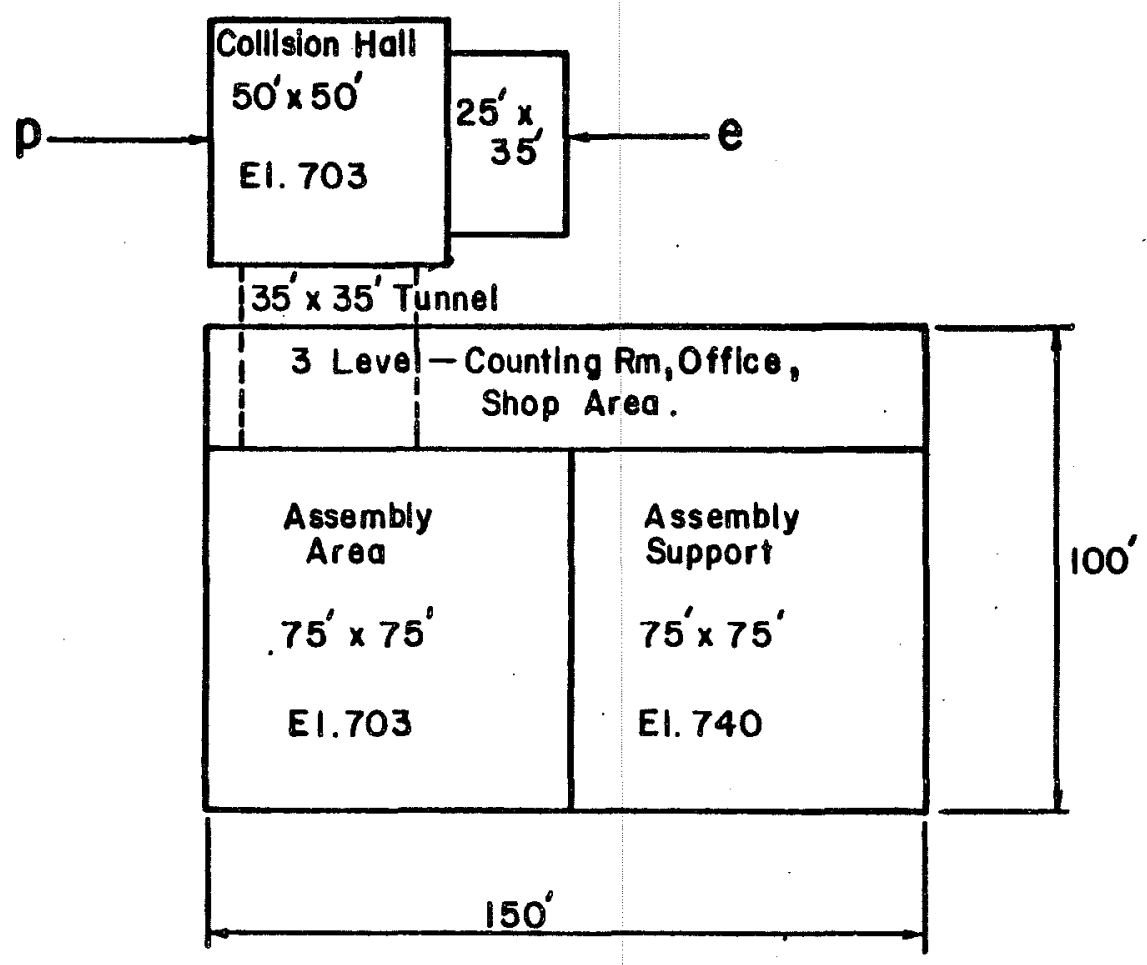

Fig. VI-7 
In addition to the collision halls, the standard tunnel sections are interrupted by occasional special enclosures:

1. Utility straight sections: From the point of view of conventional construction, the west and north utility sections present no special problem. The tunnel need not be widened or otherwise modified. The pattern of access points (to be described below) need not be changed. The east utility section is interrupted by the two ep collision halls, but the remaining portion of the straight sections will again be standard tunnel. The south utility section is the injection region, common with the Main Ring tunnel, and obviously requires modification. This will be described separately in subsection 4 .

2. Cryogenic Service Buildings: As discussed in Chapter IV, 24 satellite refrigerators similar to those used in the Saver are located around the ring, with one extra for each collision region. This means there will be 20 service buildings and penetrations into the tunnel, each with a personnel access stairway. Beam instrumentation and control electronics, power supplies, and miscellaneous auxiliary components will also be housed in these buildings. The size of these enclosures is $30^{\prime} \times 40^{\prime}$.

3. Emergency exits: Exits to the surface are provided every 900 ft; this requires one additional stairway between each satellite refrigerator.

4. Modification of straight section E: The present enclosure for the $E$ straight section will be removed and replaced with an $8^{\prime} \times 20^{\prime} \times 300$ ' common enclosure for the DC, Main Ring, Saver, and transfer lines, with a $30 \cdot \times 100$. transfer gallery above. A possible layout is shown in Fig. VI-8. This construction will necessarily create an interruption in Saver operation. The construction problem is similar to that of the penetration for the $\bar{p}$ injection line into the main ring tunnel at F17 for the TeV I project. Based on that analysis, the estimated Saver shutdown time needed to construct the EO enclosure is no more than six months.

\section{F. Roads and Services:}

1. Roads. The principal access to the DC is through the four $p \bar{p}$ collision halls and the two ep collision halls. It is therefore not clear that a major perimeter road is necessary. On the other hand, such a road would clearly be sufficient to serve the equipment and a cost estimate made on that basis is straightforward. New 24-ft paved main roads can provide access to collision halls, while 14' gravel service roads would suffice for access to the cryogenic service buildings. Only $8000 \mathrm{ft}$ of paved road and $35,000 \mathrm{ft}$ of service roads are required in this example. The estimated cost is $\$ 3.3 \mathrm{M}$. 




Layout of Enclosure at Main Ring Straight Section E (Injection to DC)

Fig. VI-8 
2. Utility Distribution: The largest power users are the experimental areas, the central helium liquefier, the ep ring, and the RF section. In addition to these lumped power users there is a general need for distributed power around the entire ring at the twenty service buildings. The total power installed is $38 \mathrm{MW}$ for the dedicated collider with an additional $18 \mathrm{MW}$ required for the ep area. The distribution is shown in Table VI-3.

\section{Table VI-3. Primary Power Distribution}

Central Helium Liquefier

(4-1.5 MVA)

6.0 MVA
$6.0 \mathrm{MVA}$
$4.5 \mathrm{MVA}$
$1.5 \mathrm{MVA}$
$4.5 \mathrm{MVA}$
$4.0 \mathrm{MVA}$
$10.0 \mathrm{MVA}$

2-Medium Experimental Areas

( $2 \times 2-1.5$ MVA)

Large Experimental Area

(3-1.5 MVA)

Small experimental area

(1-1.5 MVA)

RF (3-1.5 MVA)

4 Utility Straight Sections

( $1.0 \mathrm{MVA})$

Cryogenic Service Bldg.

(20-0.5 MVA)

EO Transfer Hall

(1.5 MVA)

$\frac{1.5 \mathrm{MVA}}{38.0 \mathrm{MVA}}$

2 ep Experimental Areas

ep Ring

6.0 MVA

$12.0 \mathrm{MVA}$

$\overline{18.0}$ MVA

Such a large quantity of power requires ponds for cooling systems. Casey's Pond, located near the end of Road A, is adequate to serve as the cooling pond for the northern quadrant of the ring. As discussed above, Lake Law must be drained to construct the ep ring and experimental areas, but a new lake must be dug near that site to provide sufficient cooling for the eastern quadrant. Existing lakes inside the present Main Ring will provide cooling for the southern quadrant. A new pond near Indian Creek must be dug for cooling for the experimental area and RF section located in the southwest quadrant. A small pond north of Pine Street may be needed for the west utility straight section. The ponds are connected by a ring distribution network to all buildings and provide fire protection as well as cooling water. Drinking water, sanitary sewer systems and septic fields are provided only at the experimental areas.

3. Office and shop space: The six new collision halls plus the vacated $\overline{B O}$ building should provide space for over 200 persons, which is 
consistent with the expected increase of laboratory personnel needed for the project, as estimated in Chapter VIII. Any additional space needed will be provided by expansion of the Central Laboratory area. This should not entail any major new construction and no allowance is made in the cost estimate for additions to the Central Lab area.

\section{G. Environmental, health, and safety considerations:}

Potential environmental health and safety concerns have been assessed. Problems inside the tunnel appear to be resolvable by conventional solutions, such as radiation-safety and electrical-safety interlock systems similar to those presently in use at Ferrilab, all of which will be described in a Project Safety Analysis Report for a low-hazard facility (as defined by DOE Order 5481.1A).

Possible problems outside the tunnel have also been considered. In examining these questions, it is prudent to consider the uItimate long-range use of the facility. For the purposes of the study, an upgraded ep collider was assumed, with 750 bunches of $4 \mathrm{TeV}$ circulating protons, at $10^{11}$ protons per bunch, colliding with $50 \mathrm{GeV}$ electrons.

The following potential hazards have been identified and studied:

1. Low-energy hadron radiation (primarily neutrons) emerging from the radiation shields that cover the collider rings:

Assuming a beam elevation of $723.5 \mathrm{ft}$, and a shielding berm elevation of 752.5 ft (the same as the present Main Ring berm), then the "worst-case" accident of proton-beam loss, repeatedly for 1 hour, will lead to a sufficiently low hadron ( $1 . e .$, neutron) radiation level ( $<10 \mathrm{mrem} / \mathrm{hr}$, while the accident condition persists) at any point around the ring that no precautions such as roped-off or fenced-of $f$ areas above ground will be required.

2. Above-ground muons from the beam aborts in the north and south utility straight sections reaching the site boundary:

Assuming a very high rate of beam aborting (at utility $N$ ) or beam dumping (at utility $S$ ), namely once per hour, then the muon radiation reaching the site boundary, even for a full-intensity proton ring at $4 \mathrm{TeV}$, will be substantially below the federally allowed limit of 170 mrem/year.

3. Muons reaching homes in the Fermilab Village from beam losses in the NE collision hall or the arcs near Batavia Road:

Assuming that the full beam is lost for one hour at the NE collision hall or for one pulse in the curved section near Batavia Road (full beam loss will, at the least, cause a magnet quench), then the muon radiation reaching the Village will be below the federally allowed limit of $170 \mathrm{mrem} / \mathrm{year}$. 
4. Environmental concerns:

An Environmental Assessment will be prepared for review by the Department of Energy. Ingredients to be included in the environmental assessment statement are the following: the new tunnels and berms, changes made to the water systems at Lake Law, Indian Creek, and in the vicinity of Casey's Pond, and radiation crossing the site boundary (see item (b) above). It is believed that a conclusion of 'no significant environmental impact' will be reached.

5. Life-safety concerns:

The DC will present no unusual safety questions that are not already commonplace and managed at Fermilab. Determination of the spacing of emergency exits in the tunnel for the case of fire, smoke, oxygen-deficiency hazards, or other emergencies will be re-evaluated. The spacing assumed in this design is approximately $900 \mathrm{ft}$, which is consistent with present practice at Fermilab and in other storage rings. 
VII. costs

The cost analysis for the Dedicated Collider is based as much as possible on Saver experience. In addition, experience with $\mathrm{TeV}$ II construction provides a reliable baseline for costs associated with the conventional construction. It should be noted that these and the other major Saver costs, magnets and refrigeration, are very well known while others, such as controls, are less well known and more difficult to extrapolate into the future. The cost summary is shown in Table VII-1, and what follows are brief descriptions of how the numbers are obtained. AII cost rigures are in FY83 dollars. EDIA and contingency are independently estimated for the individual itemizations. No estimate of escalation has been made.

\section{A. Magnets}

Costs for standard DC magnets are shown in Table VII-2. All conductor, coil, cryostat, yoke, and labor costs are included in these figures. They have been computed from known Saver production figures (FY81).

The projection from FY 1981 to FY 1983 is based on DOE's composite inflation rate which is a weighted average of operating, plant, and equipment. For 1981 to 1982 the rate is $7.6 \%$ and for 1982 to 1983 the rate is 7.3\%. All costs excluding conductor and labor are therefore inflated by $16 \%$. Even though conductor costs have been nearly constant over the past few years (due to cancellation of higher production cost against lower raw-material cost), an inflation of $8 \%$ was used to be safe.

The parts costs during production are known extremely well for the Saver. The labor for the various parts of the magnets is also well known. Less well known are the costs attributed to $R$ \& $D$ and various kinds of overhead. Since, however, the total costs for magnets from gross budgeting numbers are well known, the costs associated with $R \& D$, tooling, etc. can be inferred. These differences are typically 10-15\% for dipoles, but considerably larger for the less costly quadrupoles and spool pieces. Salaries for managers, engineers and drafting are also included in the unit costs. 


\section{Table VII-1. Cost Summary (FY 83 Dollars) for p $\bar{p}$ Option}

\section{Technical components}

Magnets

Refrigeration

Accelerator Systems

\section{Conventional Facilities}

p $\bar{p}$ Storage Ring Facilities

Experimental Halls and Assembly Areas

Testing and Installation

EDIA

Technical Components*

Conventional Facilities

\section{Contingency}

Technical Components

Conventional Facilities

Installation \& Testing

$$
\begin{aligned}
& \begin{array}{l}
\$ 107.6 \mathrm{M} \\
27.4 \\
32.5 \\
\hline \$ 167.5 \mathrm{M}
\end{array}
\end{aligned}
$$

69.7

35.2

$\$ 104.9 \mathrm{M}$

$\$ 14.5 \mathrm{M}$

16.5

15.8

Total

20.6

18.1

3.6

$\$ 42.3 \mathrm{M}$

$\$ 361.5 \mathrm{M}$

Magnet-associated EDIA is included in the cost of magnets. 
The proposed DC has longer dipoles and quads so that the unit cost must be scaled appropriately. The dipole is $21 \%$ longer. The quadrupole is $49 \%$ longer. The scaling is applied only to "length" related costs (as opposed to "end" related costs). Spares and rebuilds of plawed magnets are assumed to add $15 \%$ to the cost.

Table VII-2. Magnet Costs

Dipoles (1168)

Quadrupoles (332)

Spools (332)

Low-B Quadrupoles( 32 )

\begin{tabular}{r} 
Unit cost \\
\hline$\$ 53.6 \mathrm{~K}$ \\
$38.5 \mathrm{~K}$ \\
$31.3 \mathrm{~K}$ \\
$77.0 \mathrm{~K}$
\end{tabular}

Total cost

$\$ 62.6 \mathrm{M}$

$12.8 \mathrm{M}$

$10.4 \mathrm{M}$

$2.5 \mathrm{M}$
Total (with spares and rebuilds)

$\$ 72.0 M$

14. $7 \mathrm{M}$

$12.0 \mathrm{M}$

$\frac{2.8 \mathrm{M}}{\$ 101.5 \mathrm{M}}$

Contingeney (5\%)

$5.1 \mathrm{M}$ $\$ 106.6 \mathrm{M}$

Special elements

$6.1 \mathrm{M}$

EDIA (25\%)

$1.5 \mathrm{M}$

Contingency $(25 \%)$

$7.6 \mathrm{M}$

$\frac{1.9 M}{\$ 9.5 M}$

Magnet Total

$\$ 116.1 \mathrm{M}$

Included in the category "special elements" are miscellaneous special beam-correction elements, the electrostatic separation system, injection, extraction, abort kickers, and septa. The cost of these elements is less well known. To the cost estimate we add 25\% EDIA, and to that 25\% contingency. For the "conventional" magnets, the EDIA has been included in the magnet cost estimate. The $5 \%$ value for contingency is justified by the experience from the just-completed Saver project, and the anticipation that simplification in design and more efficient fabrication will in fact decrease the costs (see Chapter IX).

\section{B. Refrigeration System}

The cost estimate for the refrigeration system can be made quite reliably from Saver experience as well as from the recent BNL experience with commercial vendors. The estimate given here has been supported by a cost study provided by Koch Process Systems, Inc. The cost breakdown is shown in Table VII-3. 
Table VII-3. Refrigeration Costs

\author{
Central Helium Liquefier II \\ $\$ 6.4 \mathrm{M}$ \\ Central Satellite Compressor Station \\ 2.8 \\ 30 Satellite Refrigerators \\ 7.4 \\ Interconnecting Piping \\ and Inventory Storage \\ 10.8

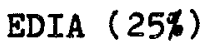 \\ $\$ 27.4 \mathrm{M}$ \\ Contingency (10\%)

$\frac{6.9}{\$ 34.3 M}$
$\frac{3.4}{\$ 37.7 M}$

Costs of the buildings housing the central helium liquefier, compressors, and satellite refrigerators are enumerated separately. This is also the case for the costs of instrumentation, control and installation.

\title{
C. Conventional Construction
}

As already mentioned, cost estimates for the DC conventional construction are inferred, whenever possible, from $T e V$ I and $T e V$ II experience. A summary of the conventional-construction costs for the $p \bar{p}$ ring is given in Table VII-4.

An $8 . \times 9$ ' tunnel of rectangular cross-section has been assumed. Allowance has been made in the cost estimate for occasional poor soil conditions and elevation variations. An additional \$3M cost penalty is included for soft tunnelling through Mt. Minooka. The estimated unit cost for the ring enclosure varies from $\$ 5 \mathrm{M} / \mathrm{mile}$ for out-and-fill in good till to $\$ 7.5 \mathrm{M} / \mathrm{mile}$ for the soft tunnelling. There is much opportunity for downward refinement of this estimate and for use of economies of scale. The tunnel cost estimated here is very conservative.

Included in the ancillary buildings are emergency exits, satellite refrigerator utility buildings and penetrations, the injection enclosure spanning Saver straight section $E$ and the DC south utility straight section, and the enclosure and penetrations for the RF system.

Road layouts and costs are discussed in Chapter VI. Site preparation costs include soil sampling, surveys, drainage, relocation of watercourses, reconfiguration of Lake Law and formation of cooling ponds, and land clearing. 
The costs of collision halls and assembly areas are taken from the direct experience of the $\mathrm{TeV} I$. BD project. The greatest uncertainty here lies in the ultimate specifications for the areas, as determined via consultation with the user community.

For the parameters given in Chapter VI, the collision-region hall and assembly area costs are given in Table VII-5. A summary of the collision hall and assembly area costs, including EDIA and contingency, is given in Table VII-6. The total collision-hall cost for the pp option (including pp assembly areas) is therefore $\$ 46.6 \mathrm{M}$. The total collision-hall cost for the ep option (omitting pp assembly areas but ine luding ep assembly areas) is $\$ 29.9 \mathrm{M}$.

Table VII-4. DC Conventional Construction Costs

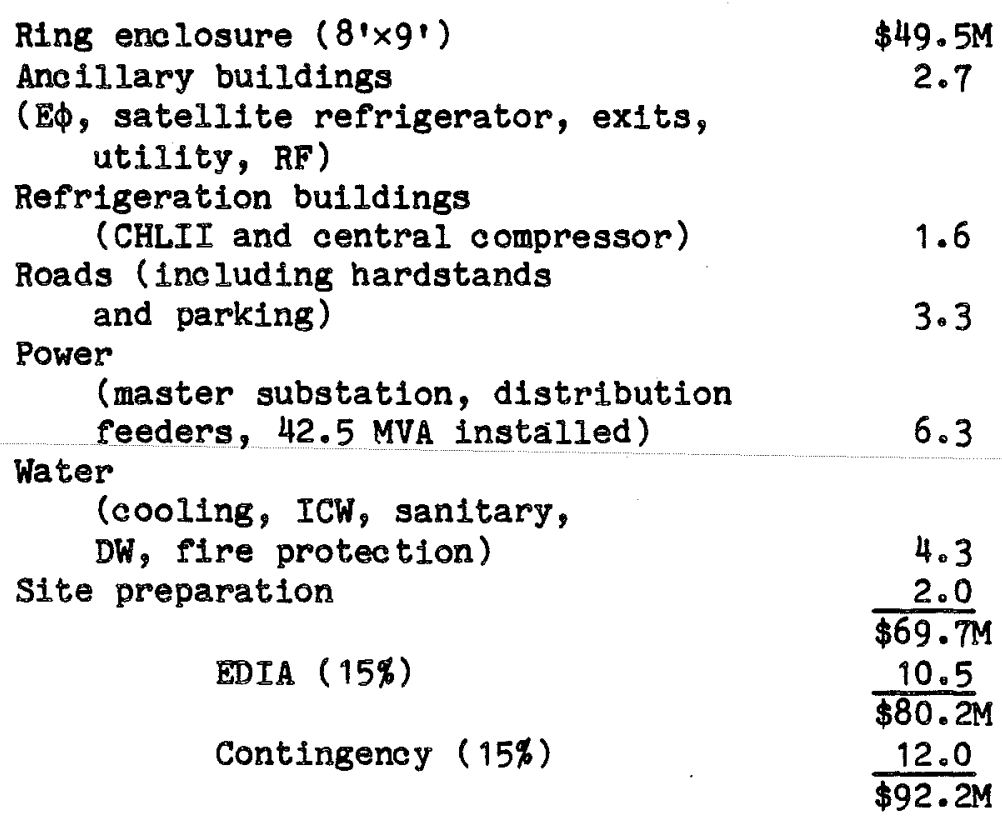

Table VII-5. Conventional Construction Costs: Experimental Halls

$\begin{array}{ccc}\text { Collision } & \text { Subgrade } & \text { Above-grade } \\ \text { Halls } & \text { Ass'y Areas } & \text { Ass'y Areas }\end{array}$

Total

\begin{tabular}{|c|c|c|c|c|c|c|}
\hline Med I & $\$ 2.8 \mathrm{M}$ & 1.9 & & 2.3 & & 7.0 \\
\hline Med II & 2.8 & 1.9 & & 2.3 & & 7.0 \\
\hline Large & 4.2 & 2.8 & & 3.0 & & 10.0 \\
\hline Smali & 2.8 & 1.9 & & 2.3 & & 7.0 \\
\hline ep I & 2.1 & & 1.4 & & 1.5 & 5.0 \\
\hline ep II & $\frac{2.1}{0}$ & & 1.4 & & 1.5 & 5.0 \\
\hline & $\overline{16.8}$ & $\overline{8.5}$ & $\overline{2.8}$ & $\overline{9.9}$ & $\overline{3.0}$ & $\$ \overline{41.0 M}$ \\
\hline
\end{tabular}


Table VII-6. Experimental Hall Cost Summary

Collision Halls pp Ass'y Areas ep Ass'y Areas

$\begin{array}{lrrr}\text { Construction costs } & 16.8 & 18.4 & 5.8 \\ \text { EDIA (15\%) } & \frac{2.5}{19.3} & \frac{2.8}{21.2} & \frac{.9}{6.7} \\ \text { Contingency (15\%) } & \frac{2.9}{22.2 \mathrm{M}} & \$ \frac{3.2}{24.4 \mathrm{M}} & \frac{1.0}{7.7 \mathrm{M}}\end{array}$

\section{Accelerator Systems}

The costs are summarlzed in Table VII-7. The control system of the Linac, electron ring and collider ring is concentrated mainly in the interface between the computers and the equipment being controlled, i.e., in CAMAC, Multibus and physical interconnection. The requirement for four PDP-11's, a VAX computer and two consoles does not seriously invalidate the assumption that the cost of controls be assumed proportional to the number of machine elements, and a cost twice that incurred in the Tevatron can therefore be assumed. A rough estimate for the Saver controls gives $\$ 3.3 \mathrm{M}$, of which approximately $40 \%$ goes to Saver ring controls and another $40 \%$ to computer. For the purposes of this study, this figure is doubled. To this is added the estimated cost of the cryogenic control system to obtain the quoted figure.

The category "electrical" includes estimated costs for magnet power supplies, correction-coil power supplies, the quench protection system, and low- $B$ quadrupole power supplies. These are all scaled from Saver experience.

The "mechanical" category includes estimates for stands, alignment equipment, and other miscellaneous hardware. The total cost has been projected from the Saver Project Cost Review (12/9/81), together with consultation with those involved in the Saver installation.

The cost of the vacuum system is easily scaled from Saver experience. Installation cost is included in the "installation" category. 
Table VII-7. Accelerator System Costs

$\begin{array}{lc}\text { Instrumentation \& Controls } & \$ 8.6 \mathrm{M} \\ \text { Electrical } & 9.5 \\ \text { Mechanical } & 6.0 \\ \text { Vacuum } & 4.4 \\ \text { AF } & \frac{4.0}{32.5 \mathrm{M}} \\ \text { EDIA (25\%) } & \frac{8.1}{} \\ \text { Contingency (25\%) } & \$ \frac{10.6 \mathrm{M}}{40.2} \\ & \$ \frac{10.2}{50.8 \mathrm{M}}\end{array}$

The RF system is similar to the Saver system, and again costs can be reliably soiled.

\section{E. Testing and Installation}

The total operating cost of the Magnet Test Facility is about $\$ 3$ million/year. It is expected that the measurements of the magnet (including spares) will require full operation for about $21 / 2$ years. It is assumed that it takes 100 man-years to install the machine in the tunnel. Another $\$ 1.8$ million is added for installation equipment. These installation costs include all machine components (magnets, stands, vacuum, utilities, RF, etc.) except those associated with the cryogenic system. These have been estimated independentiy is \$2.2M. This number is added to the others to obtain the final figure.

These costs are summarized in Table VII-8.

Table VII-8. Testing and Installation Cost

Magnet Test Facility

Installation

Contingency (25\%)
$\$ 7.5 \mathrm{M}$

$\$ \frac{7.0}{14.5 M}$

$\frac{3.6}{18.1 M}$

F. R\&D Costs

Major R\&D costs will involve magnet prototype and tooling improvements, special magnets, kickers, septa, electrostatic separators, and nuinerous small devices, e.g., beam monitors. We estimate the cost of R\&D to implement the proposal as given here at $\$ 4 M$ expended over the FY 84-5 period. This is included in the cost charts of Tible VIII-1. 


\section{G. The ep Facility}

The estimated costs for the ep ring are shown in Table VII-9. The cost includes all components of the 10-GeV electron ring and 5-GeV booster. We assume that five 10-ft sections of the Mark III linac from HEPL are available for use as a source of electrons and positrons for the booster ring. Cost estimates have been made both by explicit calculation and by scaling from CESR and PEP. The CESR and PEP costs are well documented. All costs are in FY'83 dollars.

Table VII-9. Electron Ring Costs

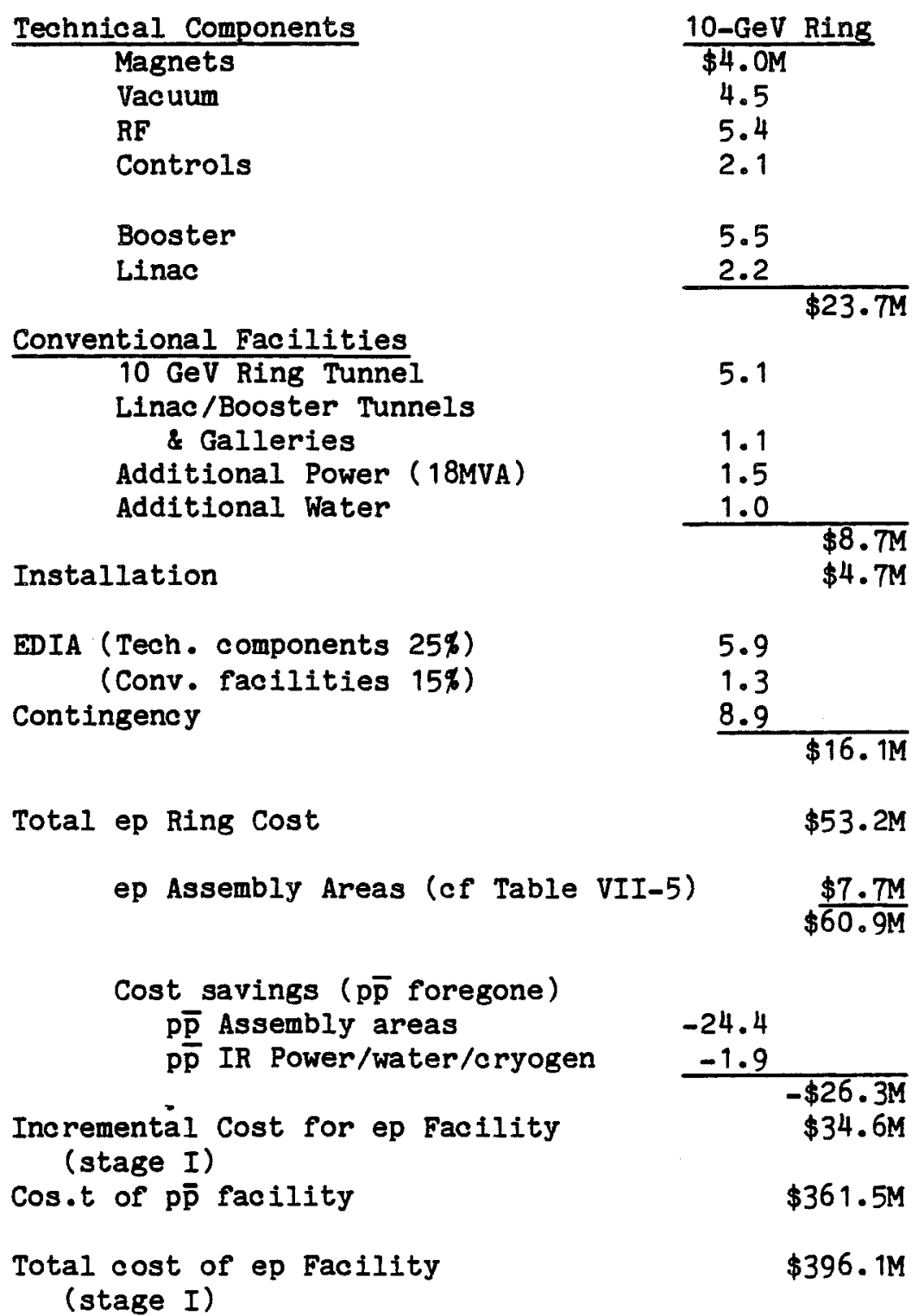


In arriving at the incremental cost for the ep facility we have, consistent with the overall design philosophy, assumed that the $p \bar{p}$ option will not be immediately implemented. This allows the cost of $p p$ assembly areas and ancillary utilities to be subtracted from the initial project cost.

\section{H. Operating Costs}

The incremental operating costs of the Dedicated Collider can be estimated using current experience for each of the following categories:

1. Power - The average Fermilab power cost for the past twelve months has been $\$ 0.045 / \mathrm{kW}$ hour. This average number can be used in the estimate in order to eliminate variations due to the complex commercial high-use rate structure.

The total power requirement is a combination of refrigeration and pump power which must remain on at all times whether the collider is operating or not (estimated at $70 \mathrm{GW}$-hrs per year) and the experimental area power which is only required during collider operation (estimated at $108 \mathrm{Gw}$ hrs for 6000 hours of collider operation per year). Thus the annual power cost for the Dedicated Collider is $\$ 8.1 \mathrm{M}$ in 1983 dollars.

2. Consumables - Helium and Nitrogen are required to make up for losses in the refrigeration system. Assuming a helium loss rate of $3 \%$ per day in an inventory of 80,000 liters, make-up helium will cost $2.1 \mathrm{M}$ per year plus liquid $\mathrm{N}_{2}$ at $\$ 0.9 \mathrm{M}$ per year.

3. Replacement Parts - Assuming a replacement part failure rate of 1\% per year, a total replacement cost of $\$ 2 M$ would be required for each year of operation.

4. Personnel costs - It is estimated that fifteen operators will be required to operate the Dedicated Collider. These operators will be assigned to five operating crews consisting of three persons per crew. In addition a systems group consisting of physicists, engineers and technicians will be required to carry out accelerator studies, plan maintenance and improvement programs and tend to the proper care and feeding of the machine. Since the other Fermilab accelerators require about ten persons per group and there is roughly twice as much equipment needed for the Dedicated Collider, it is assumed that twenty persons will be needed in the DC systems group. Other support activities will be supplied by the Accelerator Division support groups.

Assuming four experimental areas manned by ten persons each requires forty operating persons, not associated with experiments. In addition, we would assign each area a group of ten people to directly support the experimental apparatus. Thus the support groups for $\mathrm{pp}$ areas total 80 people. We make the educated guess that we will require a crew of 50 cryogenic technicians and engineers to maintain the refrigeration systems. 
These personnel costs will accumulate as follows:



The operating costs for the Dedicated Collider ( $\bar{p} p$ option) are then estimated to be:
1. Power
2. Consumables
3. Replacement parts
4. SWF
5. M\&S
6. G\&A
Total operating costs

$\$ 8.1 \mathrm{M}$

\begin{tabular}{l}
$\$ 8.1 \mathrm{M}$ \\
3.0 \\
2.0 \\
7.2 \\
$\frac{4.1}{\$ 24.4 \mathrm{M}}$ \\
6.0 \\
\hline
\end{tabular}

Capital-equipment funds as well as accelerator improvement cunds to carry out normal improvements are estimated at \$5-10M per year.

If the ep option is chosen, the operating costs will change for various reasons. Operation of the electron ring requires an estimated extra 10MW of power. There are fewer collision halls, with savings in personnel and operailing costs of approximately 40\%. Operation and maintenance requirements of the linac, booster, and $10 \mathrm{GeV}$ electron ring can be scaled from PEP and CESR experience. We estimate thit, 25 persons are required. These incremental operating costs are summarized in Table VII- 10.

Table VII-10. Incremental Operating Costs for the el gotion

Power (10)

Collision Hall Savings

Ring Operation \& Maintenance
$\$ 2.6 \mathrm{M}$

$-1.0 \mathrm{M}$

$+1.2 \mathrm{M}$

$\$ 2.8 \mathrm{M}$ 
VIII-1

VIII. PROJECT MANAGEMENT AND STAFFING

\section{A. Laboratory Priorities}

The first priority for Fermilab in the period beyond 1985-1990 must be exploitation of the Tevatron for fixed-target and colliding-beam physics. It is clear that

(i) operation and optimization of the performance of the Energy Saver,

(ii) construction, implementation, and improvements to the $\bar{p}$ source in order to attain as high a luminosity as possible, and

(iii) development of $1 \mathrm{TeV}$ beam lines and adequate support for the experiments that will use them

will require the attention of a dedicated, competent staff. Despite these constraints, and without significant numerical growth in the Laboratory manpower, it is feasible to mount the effort needed to build the DC within the schedule projected herein. The fundamental question will be our ability to identify a relatively small number of key designers and managers from cadres of accelerator experts trained in this and other accelerator laboratories. In the following, we present scenarios for the design and construction, funding profile, manpower needs, and management structure.

\section{B. Design and Construction Profile}

As illustrated in Table VIII-1, the broad outlines of the schedule are as follows:

FY 84: A design team will be assembled to complete the final design and detailed cost estimate. Magnet prototypes that satisfy all the necessary criteria for the DC will be built. If possible, procurement of long lead-time items will be initiated. An active program of workshops on DC experimental opportunities and collision-hall design will be held in order to stimulate the organization of the user community into groups of strength appropriate to effective exploitation of the physics. Calls for letters of intent will be initiated during this period.

FY 85: All component designs will be made final and tooling will be developed for all production activities. Materials procurement will proceed in earnest. Some first-round decisions on letters of intent for experiments will be made in order to ensure proper estimates of collision-hall specifications as well as to further stimulate formation of hardened detector groups. 
Table VIII-I

Dedicated Collider Overview

Manpower and Cost ( $F Y^{\prime} 83$ \$) Profiles

Fiscal Year

Design

Conceptual

Accelerator

Components

Prototype Fab

Civil Const.

Production \&

Testing

Magnets

Refrig.Assy.ôlnst.

Accelerator

Magnet In stall.

Operations

Administration

Sitework 8

Utilities

Total Manpower

Funding (TPC) ${ }^{+}$

$\overline{\mathrm{p}} \mathrm{p}$ Option

Funding ep ${ }^{\circ}$

Option

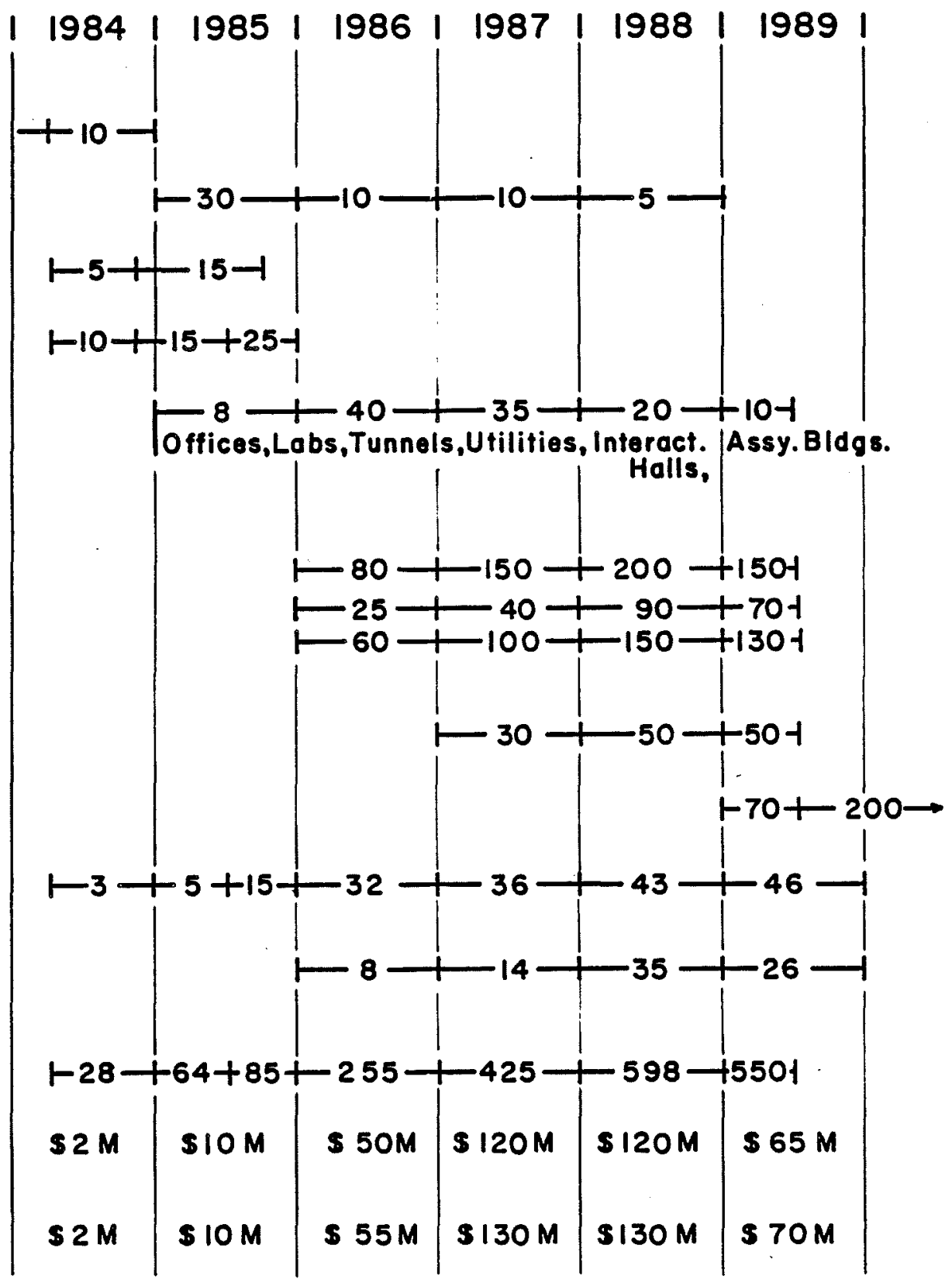

+ Collision Halls for ep Included

- Collision Halls for $\bar{p} p$ Included 
FY 86: Full-rledged conventional construction will get underway, with project organization fully in place. Production activities for components will likewise be initiated, 'in particular at the magnet factory and magnet test facility. This year will also be a major decision point for global policy issues such as ep vs.pp and first-round decisions on major experimental proposals.

FY 87-89: During this period all component fabrication and conventional construction will be completed, with installation proceeding in parallel as portions of the facility become available for occupancy. It is possible, given the funding profile, to begin commissioning the Collider in April, 1989.

\section{General Comments}

(1). Manpower Flow: The DC overview (Table VIII-1) provides our best estimates of manpower required for all DC activities. In Table VIII-2, we place the manpower requirements within the context of the overall laboratory manpower assets.

As a reference year we have chosen 1978. This was a year of good operation of the $400 \mathrm{GeV}$ program. The manpower allocations in 1978 included a substantial effort on SAVER, TeV I and TeV II R\&D. Our projected manpower requirements for the Accelerator and Research Division must include the increased operating responsibilities and the ability to continue R\&D for the Tevatron.

The build-up of 500 people in the 1978-1981 period enables us to carry out SAVER, TeV I and TeV II missions. Large numbers of physicists, engineers and technicians were transferred to follow the changing priorities of the program. It is this resource of now highly trained people which will supply the bulk of the labor required for the construction of the Dedicated Collider.

We recognize of course that treating numbers of people oversimplifies the problem. To this end, we appeal to Table VIII-3, where we have attempted to classify the skills of the presently available staff. We fully expect a mismatch of the order of $20 \%$ with a need to balance attrition, etc., with new people of required skills. This is typical of our experience over the past five years.

(2). Time scale: Given the key personnel, the most serious obstacles to achieving the 1989 completion date appear to come more from meeting the required funding profile than from uncertainties in the magnitudes of the tasks to be performed. We note that a 2-1/2 year construction period is required for magnet production. It is probably the critical path, because conventional construction, refrigeration delivery and accelerator systems development and fabrication could all fit easily with this period. To the 2-1/2 years we have added $a$ slow 
Table VIII-2

Fermilab Manpower Projections

\begin{tabular}{|c|c|c|c|c|c|c|c|c|c|}
\hline Fiscal Year & $1978^{*}$ & 1983 & 1984 & 1985 & 1986 & 1987 & 1988 & 1989 & 1990 \\
\hline D.C. & & 0 & 30 & 70 & 250 & 420 & 600 & 550 & 200 \\
\hline Accelerator Div. & $280^{*}$ & 440 & 400 & 380 & 400 & 375 & 375 & 375 & 375 \\
\hline TeV I & - & 60 & 70 & 80 & - & - & - & - & - \\
\hline Research Div. & 404 & 450 & 470 & 480 & 490 & 450 & 450 & 450 & 450 \\
\hline Physics Section & 87 & 80 & 90 & 100 & 100 & 100 & 100 & 100 & 100 \\
\hline Tech. Support & $371^{*}$ & 430 & 430 & 400 & 290 & 205 & 100 & 150 & 450 \\
\hline Sub Total Technical & 1142 & 1460 & 1490 & 1510 & 1530 & 1550 & 1625 & 1625 & 1575 \\
\hline Other Support & 422 & 540 & 550 & 530 & 520 & 500 & 500 & 500 & 525 \\
\hline Total & 1564 & 2000 & 2040 & 2040 & 2050 & 2050 & 2125 & 2125 & 2100 \\
\hline
\end{tabular}


start-up transient of six months and a contingency period of six months, giving us 3-1/2 years from Go (Oct. 1985) to Commission (April 89).

Table VIII-3. Dedicated Collider Peak Staffing Requirements;

\begin{tabular}{lcccc} 
& $\begin{array}{c}\text { Phase I } \\
\text { (FY84-86) }\end{array}$ & $\begin{array}{c}\text { Phase II } \\
\text { (FY86-89) }\end{array}$ & $\begin{array}{c}\text { 2/28/83 } \\
\text { Employment }\end{array}$ \\
\cline { 2 - 2 } Physicists & 19 & & 50 & 197 \\
Engineers & 15 & & 70 & $191(1)$ \\
Programmers & 0 & 12 & 51 \\
Drafters & 16 & 42 & $119(2)$ \\
Administrators & 11 & 46 & $162(3)$ \\
Technicians & 39 & & 393 & $831(4)$
\end{tabular}

Notes

1. Engineers include Engineering Physicists (42) and Engineering Associates (14).

2. Drafters include designers (35) and temporary (49).

3. Administration includes clerical, purchasing, personnel and all plant support persons.

4. Technicians include Tech specialists (138) Machinists (86) and welders (35).

5. These include consulting efforts obtained from other parts of the Laboratory. The full time personnel are given in Table VIII-1.

D. Management Structure

During the first phase, FY84-86, the design group will be assembled. The head of this ad hoc group will report directly to the Director. This will be a person of outstanding reputation and ability. It is expected that a large fraction of this group will continue within the formal project structure when it is institutionalized in FY86.

A vigorous effort will be made to involve non-Fermilab personnel during this initial phase. This has the following advantages:

(1) Fermilab staff will be busiest with Saver commissioning and TeV II and $\mathrm{TeV} I$ construction during this period, and 
(ii) input from a broad spectrum of the outside community is of maximal value during the early design stage.

In FY86, the formal project management structure will be fully in place, with an organization chart as shown in Table VIII-4. The administration of the Dedicated Collider will be as autonomous as practical. A good administrative model may be the CERN II management structure used to build the SPS. There will necessarily be some amount of "matrix management", where expertise present in the remaining Fermilab Divisions is used in consultative modes, especially in the early phases of design and construction.

\section{E. Profile of Staffing Requirements}

The scenario described above is naturally divided into two portions. The first phase, FY84-86, includes all conceptual design and R\&D efforts, while the second phase, FY87-89, includes all construction efforts.

The total staff requirements estimated for these phases (but not including $A \& E$ effort) are summarized below:

\section{Phase I}

EDIA

Component R\&D

Component Fabrication

Total Man Years

\begin{tabular}{rr}
80 & 425 \\
75 & 125 \\
0 & 1270 \\
\hline 155 & $\frac{1220}{182}$
\end{tabular}

The distribution of disciplines and functions can be gleaned from Table VIII- 1 and Table VIII-3. The effort at time of maximum employment is 100 for Phase I and 615 for Phase II. Approximately 35 for Phase I and 120 for Phase II are physicists and engineers. For comparison, present total Fermilab employment is approximately 2000, including approximately 385 physicists and engineers, as shown in Table VIII-3.

It may be that the technical-component effort estimated above may be somewhat high. Building all of Fermilab, including the Linac, Booster, Main Ring, Switchyard, and experimental areas, required only about 2200 man years of effort. Of this, the Main Ring required only about $480 \mathrm{man} /$ years.

\section{F. Requirements on A\&E Effort}

In the period of Fermilab design and construction, DUSAF, the Architectural Engineering firm, expended a total of about 650 man-years 
Table VIII -4

Dedicated Collider Construction Project Organization




in designing, contracting for and managing $\$ 105,240,000$ of civil construction. Thus one man-year of effort produced $\$ 160,000$ worth of construction in 1972 dollars. Integrated escalation from 1972 to 1983 is 2.444 (DOE numbers) so one would expect each man-year of $A E$ ef fort to produce $2.444 \times \$ 160,000=\$ 400,000$ of 1983 construction at the DUSAF rate.

Assuming a total of $\$ 100,000,000$ of civil construction in the DC (tunnels, utilities and experimental enclosures), 250 man years of effort or about 60 persons for four years would be required. For comparison (which is imprecise because of the differences in type of construction), the Tevatron Construction Group in the year ending on $6 / 30 / 83$ will have designed and contracted for $\$ 18,300,000$ using about thirty man-years of effort or about $\$ 610,000$ per man year of effort.

From this it would seem to the Tevatron Construction Group can be expanded to the right size to do this job.

\section{G. Funding Profile}

Project completion is projected to be 1989. This schedule depends upon a combination of PE\&D and R\&D funds in FY84 and FY85 totaling about $\$ 5 \mathrm{M}$. We have also assumed about $\$ 8 \mathrm{M}$ of construction funds to be available in FY85.

A possible funding profile is shown in Table VIII-1. This profile includes \$7M of R\&D from FY84 through FY86. 


\section{PARAMETER OPTIMIZATION}

Up to this chapter we have developed the proposal for a machine that is as well defined and specific as possible. All costs, schedules and parameters follow from this phllosophy. In this chapter we consider a range of variations from the rigorous saver duplication in the direction of improving on our experience. The schedule given in chapter VIII gives us time to carry out modest R\&D in order to validate any questionable variations from Saver practice. In particular we must provide $a$ much more refined conceptual design in time for the FY86 budget cycle (July 1984) and a complete design that will achieve a "GO" for major expenditures by fall of 185 .

The DC design we have presented is conservative. A minimal amount of R\&D for system improvement has been assumed. Although this must remain a basic guideline in the project, one must naturally expect modification and improvements. There is also a generous amount of free space available in the lattlce; the ratio of bending radius to mean radius is only 69\%. This ratio might well increase in the optimization process.

One must not assume that the cost of the project will increase owing to such inevitable "improvements" acoreted during the final design process. We illustrate this point with the following example of an austere version of the DC, which increases the energy by $15 \%$ and reduces the cost.

First, we note that the tune of the DC is quite higin. This was chosen to keep the betatron wavelength short, allowing more bunches (at one bunch per betatron wavelength) to be stored in the machine. This choice of "high" tune also implies that limitations on aperture originating from lattice nonlinearities should be lessened. This may turn out to be important because of the sinuous design orbits of the separated $p$ and $\bar{p}$ beams, despite the evidence to the contrary from explicit theoretical estimates. It is not easy to identify in advance and correctly estimate all the features of the machine that will limit performance.

The question of whether the DC design is "overconservative" is best addressed in the context of upcoming Saver performance. If the DC is overconservative one would be very tempted to decrease the tune. Examination of the cost table in Chapter VII shows considerable investment in quadrupole magnets and correction elements. Let us suppose we may decrease the tune by a factor of two, i.e. to 22 . The number of normal quadrupoles and spools is halved (although trim dipoles may be somewhat stronger). The length of each quadrupole is halved as well, because the focal strength per magnet is halved. Then enough free space is opened up to allow an extra DC dipole to be added to each cell, provided the magnetic length is reduced from $7.75 \mathrm{~m}$ to $7.55 \mathrm{~m}$. This is shown in Table IX-1. 
Table IX-1. Comparison of Cell Parameters of DC and a Possible Low-Tune Alternative

$\begin{array}{lcc} & D C & \begin{array}{c}\text { "Austere" } \\ \text { machine }\end{array} \\ \text { Half-cell length } & 37.40 \mathrm{~m} & 74.80 \mathrm{~m} \\ \text { "Slot" length of dipole } & 8.05 \mathrm{~m} & 7.85 \mathrm{~m} \\ \text { Total "slot" length of dipoles } & 32.20 \mathrm{~m} & 70.65 \mathrm{~m} \\ \text { Magnetic length of quads } & 2.50 \mathrm{~m} & 1.25 \mathrm{~m} \\ \text { Remainder for spools, position } & & \\ \text { monitors, etc. } & 2.7 \mathrm{~m} & 2.9 \mathrm{~m}\end{array}$

This implies an increase in energy of $(12.5-2.6) \%=10 \%$. In the same spirit of austerity, one might reduce the symmetry from eight-fold to six-fold, eliminating the large amount of possibly unused space in the west and north utility straight sections. In such a design, the electron ring could be located at the north utility straight section. The four remaining straight sections would be relocated slightly and would still be used for $p \bar{p}$ collisions. The only geographical problem occurs for the northwest collision hall which is deeper underground; that cost penalty is small. With these modifications, an extra fraction $2 / 44=4.5 \%$ of the circumference can be invested in bending, giving an overall increase in machine energy of $(10+4.5) \% 215 \%$. This alone leads to 4.6 TeV oms energy.

The incremental cost of this energy increase is negative! The change in the relevant magnet costs (omitting low- $\beta$ quads, etc.) is shown in Table IX-2.

Table IX-2. Cost Comparision for the DC and a Possible Low-Tune Alternative

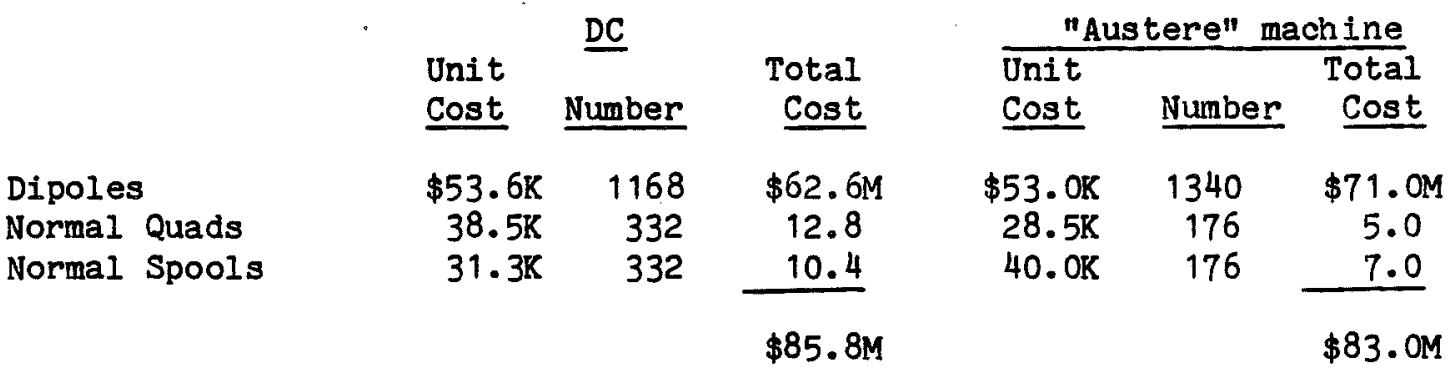

The decreased cost of quadrupoles and spools more than compensates for the cost of the extra bending magnets.

It is olear that these cost savings have been made at the expense of some growth potential and possibly of machine performance as well. Luminosities well in excess of $10^{31} \mathrm{~cm}^{-2} \mathrm{sec}^{-1} \mathrm{can}$ only be attained by storing more than one bunch within one betatron wavelength. Tnis should not be ruled out; again the issue will be what the usable machine aperture really turns out to be. 
The magnet cost estimates contained in this proposal were developed from Saver experience under the constraint that no changes in magnet design or fabrication technology requiring development ef fort were to be considered. Work underway at BNL and DESY, as well as the R\&D experience at Fermilab, suggest several new approaches to magnet design and fabrication which can be considered in the parameter optimization process that will take place berore the design is finalized. We estimate that a modest R\&D effort working in parallel with the detailed machine design could yield up to a $30 \%$ improvement in performance/cost for the DC magnet system compared with using Saver magnets.

Another obvious opportunity for increasing the DC performance is to increase the radius. While a $15 \%$ increase is geographically possible, a considerable change in the DC layout, injection lines, etc. would be implied. But a $10 \%$ increase in radius may well be possible if the cost penalty of soft tunneliling (at present not very well known) turns out to be small. The DC tunnel could then go under the Village, and a small clockwise rotation of the ring about the El injection point would avoid boundary constraints at the northwest corner of the site.

In summary, we see three options which could yield an increase in energy: i) bending via slightly stronger magnets $(\sim 10 \%)$, ii) more bending via a more tightly packed ring ( $-15 \%)$ and a larger radius $(\sim 10 \%)$. Thus it seems not unreasonable to expect to achieve $5 \mathrm{TeV}$ in the $\mathrm{cm}(+25 \%)$ by some combination of these options. It is not clear that there is a cost penalty in these changes. At worst, we face an overall cost increase of $20 \%$. The results of a year of R\&D will clarify the issue very greatly. This R\&D can begin in June of 1983 and will constitute a reorientation and moderate increase of ongoing efforts in magnet and accelerator R\&D. We estimate that such an effort would involve about a dozen people and an R\&D cost of about \$2M. 




Fig. 1 Energy Landmarks 


\section{EXECUTIVE SUMMARY}

We present here the proposal to build a Dedicated Collider (DC) at Fermilab. This is a pp collider operating at a center of mass energy of 4 to $5 \mathrm{TeV}$ with four interaction halls and a luminosity of more than $10^{31} \mathrm{~cm}^{-2} \mathrm{sec}^{-1}$. We have included a $10 \mathrm{mGeV}$ electron ring with two interaction regions to intersect the 2 to $2.5 \mathrm{TeV}$ proton ring for ep collisions. We have scheduled construction to begin in October 1985 and to have the ring complete and cold by April 1989. Figure I-1 illustrates the proposed ring siting.

\section{Physics}

Theoretical high-energy physics is at an impasse after a decade of remarkable progress. An impressive representation of theorists have publicly pleaded for experimental 1llumination especially in the effective mass range well "beyond the $W . "$ There is now a proliferation of speculations as to how to extend the standard model. Figure 1 illustrates this and emphasizes the $1-\mathrm{TeV}$ mass range as particularly rich in candidates for refining our view of the physical world. In the context of world physics, we note the attention being given to the effective mass region near $100 \mathrm{GeV}$ by LEP and SLC in $\mathrm{e}^{+} e^{-}$collisions, by the CERN collider in $\bar{p} \bar{p}$ collisions, and possibly HERA in ep collisions. There is much to be learned in this energy domain, but it seems clear that by the end of the decade there will be an urgent need to look at the 500 to $1000 \mathrm{GeV}$ range. This is the scientific motivation for the DC. We show in some detail that the high energy and good luminosity of the DC suffices to address the physics issues in the 500 to $1000 \mathrm{GeV}$ effectivemass domain.

We note that whereas TeV I does address the 200 to $400 \mathrm{GeV}$ region, it is compromised by sharing time with the fixed target program, TeV II, and by having only two interaction halls.

Decoupling the Collider physics from $T e V$ II results in a substantial gain in productivity for the fixed-target program. This will increase utilization by considerably more than a factor of two by the elimination of end effects in switching between programs. More importantly, experiments will be permitted longer dwell times in beam lines, a process that is well known to greatly increase the productivity of this kind of research.

\section{Energy}

The bulk of this proposal addresses the design and physics of a 4 TeV collider ( $2 \mathrm{TeV}$ against $2 \mathrm{TeV}$ ). However in Chapter IX we discuss options which give us considerable confidence that we can actually achieve close to $5 \mathrm{TeV}$ at little or no cost increase. 


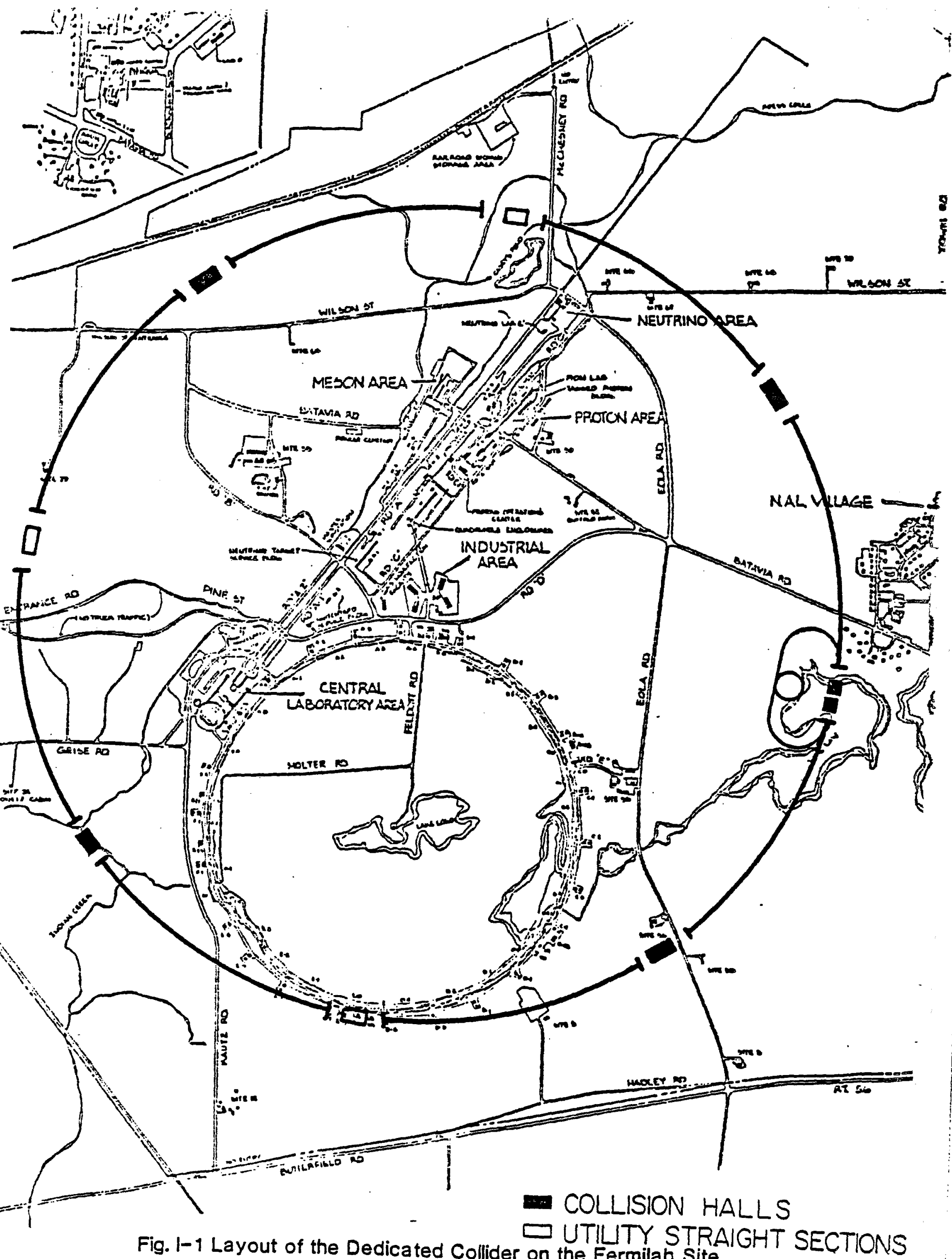




\section{Costs}

The project is being proposed in the most conservative way, following Saver experience in great detail. In this way, the costs must be overestimated, since experience usually results in improvement. Here we have ten years of experience in superconducting-magnet R\&D. This engenders a sharp discrimination between changes that may require extensive $R \& D$ and those that may be undertaken with confidence. We arrive at a construction cost of $\$ 360 \mathrm{M}$ for the $\overline{p p}$ option, with an incremental cost of $\$ 60 \mathrm{M}$ to acquire ep physics. Alternatively, we estimate a cost of $\$ 395 \mathrm{M}$ for the ep option, with an incremental cost of $\$ 25 \mathrm{M}$ for adding pp physics. To this we should add approximately $\$ 10 \mathrm{M}$ in PE\&D and R\&D costs. The choice of ep vs $\overline{p p}$ as first priority need not be made until 1986. A funding profile necessary to achieve the schedule (below) is presented in Table VIII-1. All costs are in 1983 dollars. Although we have not considered detectors in any detail, a plausible allocation for detector costs is $\$ 120 \mathrm{M}$ for the $\mathrm{p} \overline{\mathrm{p}}$ option and $\$ 50 \mathrm{M}$ for the ep option. This assumes the use of an upgraded CDF as well as the detector to be constructed for do.

\section{Schedule and Manpower}

Crucial dates are the following:

AUGUST 1983 Refined conceptual design and request for FY85 construction funding at the level of $\$ 10 \mathrm{M}$.

JULY 1984

Final design after review by DOE for FY 86 budget cycle. Completion of all R\&D essential to achieving the design.

OCTOBER 1984 Beginning of procurement of long lead-time items, tooling, and so forth. Begin A\&E work, some site preparation

OCTOBER 1985 Beginning of assembly of magnets, refrigeration. Begin civil construction.

APRIL 1989 Cooldown of entire ring and beginning of commissioning.

SEPTEMBER 1989 Pnysics

Allocation of manpower to this task will largely come from people now involved in Saver, $\mathrm{TeV} I$, and $\mathrm{TeV}$ II construction activities. Assignments to various tasks are shown in Table VIII-1. The DC manpower needs are put in the context of the Laboratory in Table VIII-2. Meticulous attention has been paid to giving the Tevatron program enough support to be able to operate and improve the Saver, beam lines, and TeV I reliability and intensity. The Laboratory manpower growth is minimal ( $5 \%$, but there will be problems of matching skills between what we have and what we need. 
This proposal is submitted to the 1983 Woods Hole Panel as a considered plan for illuminating physics in the 4 to $5 \mathrm{TeV}$ range before the end of this decade and with minimum technological risk. 


\section{Table VIII-1}

\section{Dedicated Collider Overview}

\section{Manpower and Cost (FY'83\$) Profiles}

Fiscal Year

Design

Conceptual

Accelerator

Components

Prototype Fab

Civil Const.

Production \&

Testing

Magnets

Refrig. Assy.\&inst.

Accelerator

Magnet Install.

Operations

Administration

Sitework 8

Utilities

Total Manpower

Funding (TPC) p p Option

Funding ep ${ }^{\circ}$ Option

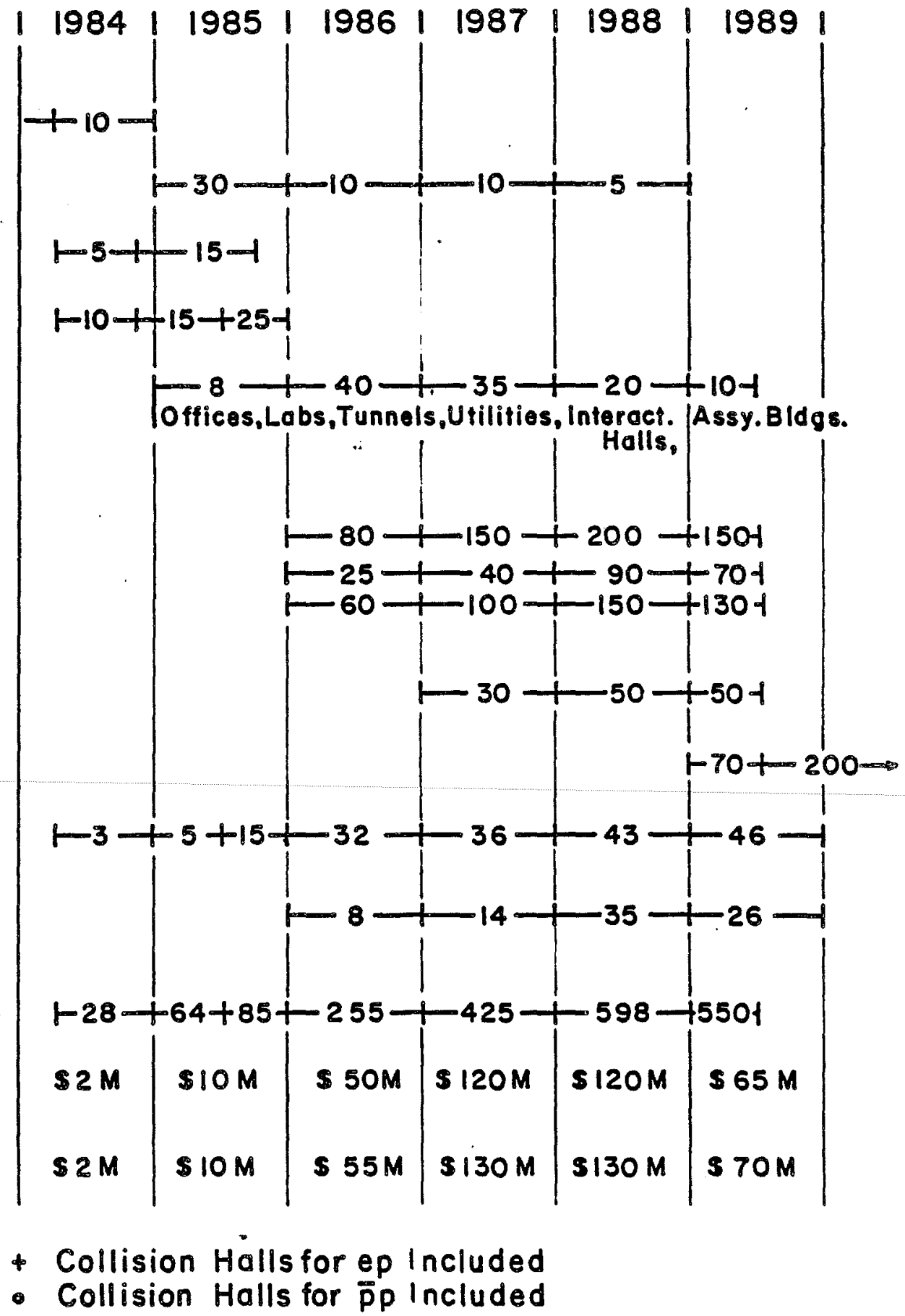


